\title{
Reflective Lorentzian Lattices of Signature $(5,1)$
}

\author{
Dissertation \\ zur Erlangung des akademischen Grades eines \\ Doktors der Naturwissenschaften
}

(Dr. rer. nat.)

vorgelegt von

Dipl.-Math. Ivica Turkalj

an der

Fakultät für Mathematik

der

technische universität
dortmund

Juni 2017 


\section{Dissertation}

Reflective Lorentzian Lattices of Signature $(5,1)$

Fakultät für Mathematik

Technische Universität Dortmund

Erstgutachter: Prof. Dr. Rudolf Scharlau

Zweitgutachter: Prof. Dr. Detlev Hoffmann

Tag der mündlichen Prüfung: 8. August 2017 


\section{Contents}

$\begin{array}{ll}\text { Introduction } & 1\end{array}$

\begin{tabular}{lll}
\hline Chapter 1. & Integral Lattices & 7
\end{tabular}

\begin{tabular}{lll}
\hline 1.1. & Fundamentals on Integral Lattices & 7
\end{tabular}

$\begin{array}{lll}1.2 . & \text { The Mass Formula } & 9\end{array}$

1.3. Positive-Definite Reflective Lattices 12

$\begin{array}{lll}\text { Chapter 2. } & \text { Classification of Totally-Reflective Lattices } & 19\end{array}$

$\begin{array}{lll}2.1 . & \text { Bounds for the Determinant } & 19\end{array}$

$\begin{array}{lll}2.2 . & \text { Reduction to the Strongly Square-Free Case } & 27\end{array}$

$\begin{array}{lll}2.3 . & \text { Classification Result } & 30\end{array}$

$\begin{array}{lll}\text { Chapter 3. Hyperbolic Reflection Groups } & 33\end{array}$

$3.1 . \quad$ Fundamentals on Hyperbolic Reflection Groups 33

$\begin{array}{lll}3.2 . & \text { Arithmetic Reflection Groups and Reflective Lorentzian Lattices } & 38\end{array}$

3.3. Methods to Prove Reflectivity and Non-Reflectivity 41

$\begin{array}{lll}\text { Chapter 4. } & \text { Classification of Reflective Lorentzian Lattices } & 45\end{array}$

4.1. Connections between Reflective Lorentzian Lattices and TotallyReflective Genera 45

4.2. Classification Result for Reflective Lorentzian Lattices 47

$\begin{array}{lll}4.3 . \quad \text { Examples } & 50\end{array}$

Index

\begin{tabular}{lr}
\hline Bibliography & 57
\end{tabular}

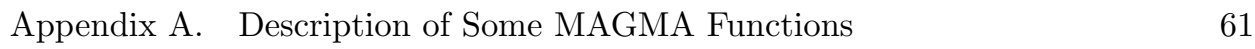

\begin{tabular}{|lll}
\hline Appendix B. & Primitive Totally-Reflective Genera of Dimension 3 & 65
\end{tabular}

\begin{tabular}{|lll}
\hline Appendix C. & Primitive Totally-Reflective Genera of Dimension 4 & 77
\end{tabular} 



\section{Introduction}

General Topic. With this work, we wish to contribute to the problem of classifying discrete groups of motions generated by reflections. These groups, certainly interesting in their own right, appear in various branches of mathematics: in Riemannian geometry as discrete groups of isometries of smooth manifolds of constant curvature, in Algebra in connection with the theory of Coxeter groups, invariant theory and representation theory, in number theory as Weyl groups of integral quadratic forms, in algebraic geometry in the study of $K 3$ surfaces and complex hyperbolic spaces, and in low-dimensional topology.

Due to the fact that there are three classes of simply-connected complete Riemannian manifolds of constant sectional curvature (two of those are standard models for non-Euclidean geometries), the theory of these groups can be divided into three areas: spherical, Euclidean and hyperbolic reflection groups. According to Bourbaki's historical notes (cf. Bou08), the modern theory of spherical and Euclidean reflection groups originates from geometrical investigations of Möbius and Schläfli in the mid 19th century, which then were extended and applied to Lie theory by Killing and Cartan around 1900. It culminates in the groundbreaking work of Coxeter who gave a complete classification of spherical and Euclidean reflection groups in Cox34. Qualitatively, his result can be summarized as follows: there are infinite series of such groups contained in each other with increasing dimension, but only a few groups in any fixed dimension. Furthermore, the combinatorial structure of a fundamental polyhedron is simple in the sense that it is always a
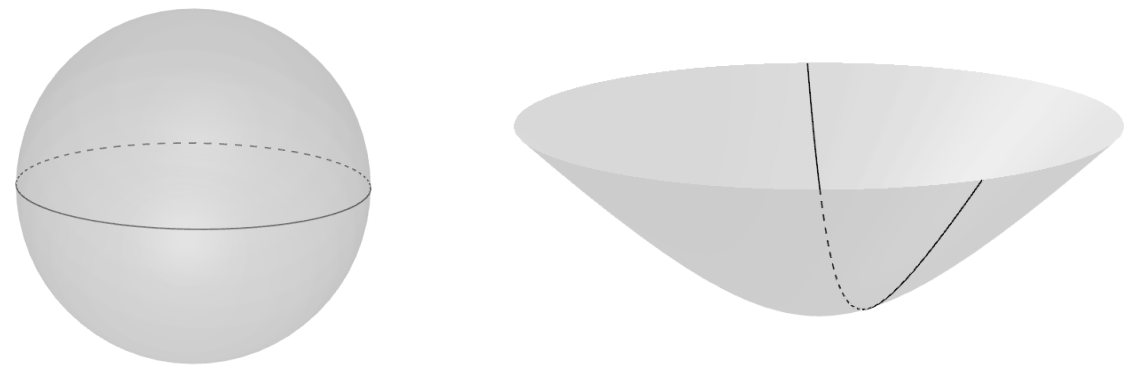

FiguRE 1. Reflection hyperplane (black curve) in the spherical and hyperbolic space of dimension 2 . 


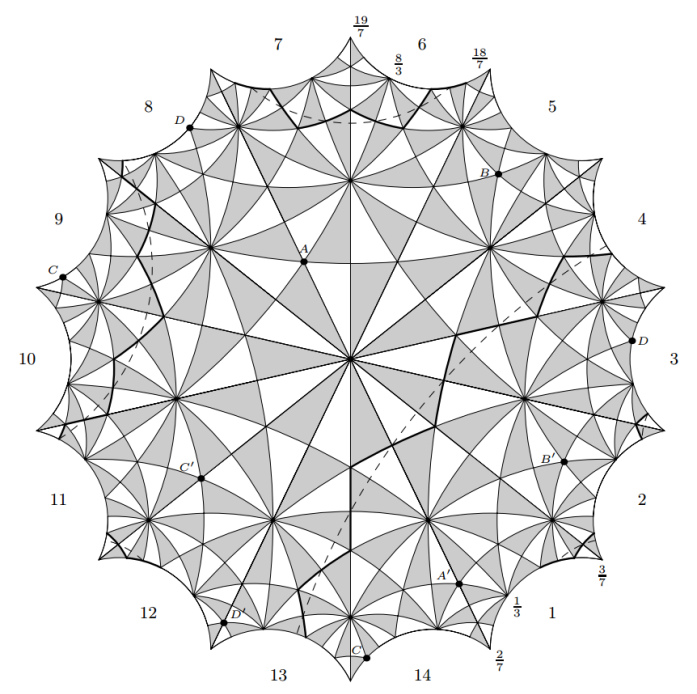

Figure 2. A tessellation of (a domain of) the hyperbolic plane by the fundamental polyhedron of the $W(2,3,7)$ reflection group (see example 3.4 for the definition of $W(2,3,7)$; image taken from Kle79]).

product of simplices. A classification of similar generality is not known for hyperbolic reflection groups and is probably not within reach of current research. The behavior of these groups is fundamentally different. In large dimensions there are no groups at all; there are no infinite series in particular. On the other hand, a lot of groups appear in small dimensions which then have a fundamental polyhedron with a complicated combinatorial structure.

In the following we will recap what is established so far. The classification of hyperbolic reflection groups of finite covolume is known in principle for dimension 2 (cf. Poi82 ) and 3 (cf. And71a, And71b). The groups in Poi82 play an important role in the work of Klein on discrete groups of isometries of the hyperbolic plane (cf. [Kle79]). It was shown by Vinberg and Prokhorov (cf. [Vin81, [Vin84, Pro86]) that groups with finite covolume do not exist in dimension $\geq 996$. If one sharpens the condition to the fundamental domain and considers groups with a compact polyhedron, then reflection groups only exist in dimension $<30$ (cf. Vin81, Vin84]). There is no reason to expect these bounds to be sharp.

The most interesting subclass are the so-called arithmetic hyperbolic reflection groups. These groups always have a fundamental domain of finite volume, which can be compact or non-compact. To sketch the notion of arithmeticity, consider a totally real number field $F$ and its ring of integers $\mathfrak{o}_{F}$. Let $E$ be a $\mathfrak{o}_{F}$-lattice of signature $(n, 1)$ such that for every non-identity embedding $\sigma: F \longrightarrow \mathbb{R}$ the lattice ${ }^{\sigma} E$ is positive-definite. The isometry group $O^{+}(E)$ can be considered as a discrete 
subgroup of the full isometry group of the hyperbolic space of dimension $n$. Results of Borel \& Harish-Chandra (cf. BH62 ) imply that $O^{+}(E)$ has finite covolume. Finite-index subgroups of groups obtained in this manner are called arithmetic. By definition, the group $O^{+}(E)$ is always arithmetic. If it is generated up to finite index by reflections, then the lattice $E$ is said to be reflective.

Let us first consider the non-cocompact case. From [BH62 it follows that an arithmetic group has a non-compact fundamental domain if and only if $F=\mathbb{Q}$ and $E$ is isotropic. By the theorem of Hasse-Minkowski, the latter condition is automatically fulfilled if $F=\mathbb{Q}$ and $n \geq 4$. Thus, for $n \geq 4$, an arithmetic group is non-cocompact iff the field of definition is $F=\mathbb{Q}$. Vinberg showed in Vin81, that non-cocompact arithmetic reflection groups only appear in $n<30$. The coincidence of the dimensions in this and the previously mentioned bound for not necessarily arithmetic reflection groups with compact fundamental domain is accidental. A sharp result was proven by Esselmann (cf. [Ess96]) who showed that the dimensions of non-cocompact arithmetic reflection groups satisfy $n \leq 21, n \neq 20$. His theorem is sharp due to the well-known example of Borcherds (cf. Bor87]). Examples for $n \leq 19$ were found earlier by Vinberg (cf. Vin72a) and Vinberg \& Kaplinskaya (cf. VK78). Full classifications have been carried out by Scharlau and Walhorn, who have listed all maximal arithmetic reflection groups with non-compact fundamental domain in dimension 3 (cf. [Sch89]) and 4 (cf. Wal93).

Regarding arithmetic reflection groups with compact fundamental domain, it was established through a series of paper by Nikulin ([Nik82, [Nik09], [Nik11]) in combination with [Mac11] and BL14] that the degree of the field of definition $F$ is at most 25. Examples for $n \leq 5$ are given in [Mak70, Kap74 and for $n \leq 8$ in Bug84, Bug90, Bug92.

The most recent classification projects were approached in terms of reflective lattices. It was known earlier that there is only a finite number of reflective lattices if the dimension of the hyperbolic space and the degree of the definition field is fixed (cf. Nik81). Interested in their close relation to $K 3$ surfaces and Kac-Moody algebras, Nikulin classified in $\mathbf{N i k 0 0}$ all strongly square-free reflective $\mathbb{Z}$-lattices of rank 3. This work was continued by Allcock, who listed all (not only strongly square-free) lattices of above type (cf. All12). The transition to number fields was studied in [Mar15] where rank 3 reflective lattices over $\mathbb{Z}[\sqrt{2}]$ were classified.

Results and Strategies. The last-mentioned authors have in common that their approach is geometrical; by studying the shape of the fundamental polyhedron they reduce the number of candidates for reflective lattices to a manageable size. In contrast, we choose the approach of Scharlau and Walhorn (cf. [SW92]), which is heavily based on the arithmetic theory of quadratic forms and their connection to 
root systems. This method seems to be more efficient in higher dimensions $(n \geq 4)$. Using local theory we analyze the structure of reflective lattices and arithmetic reflection groups and prove the following statement.

\section{THEOREM.}

a) Let $E$ be a strongly square-free reflective lattice of signature $(5,1)$. Then $E$ is of the form $E={ }^{a} \mathbb{H} \perp L$ with $L$ a square-free totally-reflective lattice of dimension 4 and $\alpha \in\{1,2\}$. The scaling factor $\alpha=2$ only occurs if the 2-adic symbol of $E$ is of the form $\mathrm{I}_{5,1}\left(1^{\varepsilon_{0}, 4} 2_{\mathrm{II}}^{\varepsilon_{1}, 2}\right)$.

b) Let $W$ be a maximal arithmetic reflection group on the hyperbolic 5-space. Then $W$ is of the form $W=W\left({ }^{a} \mathbb{H} \perp L\right)$ with $L$ a square-free totallyreflective lattice of dimension 4 and $\alpha \in\{1,2\}$.

Here, a positive-definite lattice is called totally-reflective if every lattice in its genus has a root system of full rank and ${ }^{\alpha} \mathbb{H}$ is the $\mathbb{Z}$-lattice with Gram matrix $\left(\begin{array}{cc}0 & \alpha \\ \alpha & 0\end{array}\right)$. With this structure theorem and additional techniques from the general theory of hyperbolic reflection groups, we reduce the classification of reflective lattices and arithmetic groups to those of totally-reflective lattices of smaller dimension. We should mention that we are also interested in totally-reflective lattices as objects in their own right; thus the following result is more general than the requirements of the structure theorem. The strategy leading to the classification is as follows:

Step 1: Let $L$ be a strongly square-free totally-reflective lattice with $\operatorname{dim} L=4$ (resp. $\operatorname{dim}=3$ ). Hence $\operatorname{det} L$ is of the form $\operatorname{det} L=p_{1}^{2} \cdots p_{r}^{2} \cdot q_{1} \cdots q_{s}$ (resp. $r=0)$. Using the mass formula and the combinatorial description of lattices with full-rank root system by Scharlau \& Blaschke (cf. [SB96]), we prove that $r \leq 9$ and $s \leq 8-r$ (resp. $s \leq 10)$.

Step 2: Then, we show that there are bounds $c_{i}$ and $d_{j}$ (one for every prime factor) depending only on the number of prime factors such that $p_{i} \leq c_{i}$ and $q_{j} \leq d_{j}$. Thus the number of local invariants that need to be taken into account is effectively bounded and the enumeration is computationally feasible.

Step 3: After finishing the strongly square-free classification, we obtain all squarefree, primitive totally-reflective genera by partial dualization.

Step 4: The last step consists in dropping the assumption "square-free" by determining the pre-images of square-free genera under the Watson transformation.

After carrying out all the steps, we obtain the following result. 


\section{TheOrem.}

a) In dimension 3, there are 1234 primitive totally-reflective genera of which 289 are square-free and 52 strongly square-free.

b) In dimension 4, there are 930 primitive totally-reflective genera of which 230 are square-free and 88 strongly square-free.

Up to here, the procedure gives us a list of candidates for reflective lattices of signature $(5,1)$ and allow a subsequent application of an algorithm due to Vinberg. This algorithm can prove reflectivity, but unfortunately, not non-reflectivity. In practice we bypassed this problem in two ways. If one manages to embed a nonreflective lattice of smaller dimension, then results of Bugaenko (cf. Bug92) imply the non-reflectivity of the lager one. Alternatively, non-reflectivity can be rigorously checked by group theoretical methods in each particular case, for example, by detecting an infinite order symmetry of the fundamental polyhedron. At the end, we prove:

ThEOREM. There are, up to isometry, 80 strongly square-free reflective $\mathbb{Z}$ lattices of signature $(5,1)$.

Additionally, we gave various geometric invariants of the corresponding fundamental polyhedron, such as the number of faces, the number of cusps and other.

Structure of the Thesis. In chapter 1 we recall some facts about the theory of integral quadratic forms. While Section 1 deals with the local theory and the invariants introduced by Conway \& Sloane (cf. CS99]), section 2 emphasizes the analytic theory by introducing the mass of a lattice, ways to calculate it and some results concerning $L$-series. Section 3 deals with positive-definite lattices with a fullrank root system and their combinatorial properties. We recap the combinatorial classification from [SB96, and show that the isometry group of these lattices only depends on the combinatorial class of their root system.

Chapter 2 is dedicated to the classification of totally-reflective genera in dimension 3 and 4 and the four steps outlined in the general strategy. We give bounds on the prime factors of the determinant of strongly square-free totally-reflective lattices and show how the general case is reduced to this one.

The following chapter 3 is meant as an introduction to the theory of arithmetic hyperbolic reflection groups and reflective lattices. We give descriptions of hyperbolic reflection groups in terms of Coxeter diagrams and normalized Gram matrices and show how arithmeticity is connected to the theory of root systems. After discussing Vinberg's algorithm, we introduce two methods to bypass its major weaknesses. 
The last chapter clarifies the connections between arithmetic reflection groups and totally-reflective genera and presents the classification result regarding reflective lattices of signature $(5,1)$.

Acknowledgment. I would like to express my sincere gratitude to my advisor Prof. Dr. Rudolf Scharlau for the continuous support of my Ph.D studies and related research. I would also like to thank David Lorch and Markus Kirschmer for providing helpful MAGMA functions which i have used during my calculations. 


\section{CHAPTER 1}

\section{Integral Lattices}

\subsection{Fundamentals on Integral Lattices}

The goal of this first section is to introduce the basic definitions and concepts related to the theory of integral lattices. As the global and local theory go hand in hand, the basic terminology is developed over principal ideal domains. This allows us to cover the situation over $\mathbb{Z}$ and $\mathbb{Z}_{p}$ simultaneously.

Let $R$ be a principal ideal domain and $K:=\operatorname{Quot}(R)$ its quotient field. A lattice over $R$ is a pair $(L, b)$, where $L$ is a free $R$-module of finite rank and $b: L \times L \longrightarrow K$ a symmetric bilinear form. We write $L$ instead of $(L, b)$ if the bilinear form is clear from the context. As usual, $V:=L \otimes_{R} K$ means the enveloping $K$-space of $L$. By $O(L)$ we denote the isometry group of $(L, b)$. The determinant of $L$ is the determinant of any Gram matrix of $(L, b)$ which is well defined modulo squares of units in $R$.

We say that $L$ is integral if $b(L, L) \subseteq R$. An $R$-lattice is called even if $b(x, x) \epsilon$ $2 R$ for all $x \in L$, and odd otherwise. By ${ }^{\alpha} L$ we mean the lattice $(L, \alpha b)$ obtained by scaling the bilinear form $(\alpha \in K)$. An integral lattice $L$ is said to be primitive if $L={ }^{\alpha} K$, with $K$ an integral lattice, implies $\alpha \in R^{*}$. We denote by $L^{\#}$ the dual lattice of $L$ which is defined as $L^{\#}:=\{v \in V \mid \forall x \in L: b(x, v) \in R\}$. Clearly, $L$ is integral iff $L \subseteq L^{\#}$. For an integral $\mathbb{Z}$-lattice, the group $L^{\#} / L$ has order $\operatorname{det} L$ and is called the discriminant group of $L$. A lattice is unimodular if $L=L^{\#}$. More generally, for $\alpha \in K$, we say $L$ is $\alpha$-modular if $L=\alpha L^{\#}$. It is easy to see that any $\alpha$-modular lattice $K$ can be written as $K={ }^{\alpha} L$ where $L$ is unimodular (cf. Kit93, Proposition 5.2.1.).

We say a lattice is indecomposable if it is not the orthogonal sum of two non-zero sublattices. A non-zero $\mathbb{Z}$-lattice can be decomposed as

$$
L=K_{1} \perp \cdots \perp K_{r},
$$

with every $K_{i}$ being indecomposable. If $L$ is positive-definite, then such a decomposition is unique up to the order of the $K_{i}$ (cf. Kne02, Satz (27.2)).

Lattices over $\mathbb{Z}_{p}$ can be decomposed in a different manner and some effort is required to understand the gap between local and global (because the analog of the Hasse-Minkowski theorem over $\mathbb{Z}$ is false). We start with some basic definitions. Let 
$\mathbb{P}$ be the set of all prime numbers. The localization of $L$ at a prime spot $p \in \mathbb{P} \cup\{\infty\}$ is abbreviated as $L_{p}:=L \otimes_{\mathbb{Z}} \mathbb{Z}_{p}\left(\right.$ with $\left.\mathbb{Z}_{\infty}=\mathbb{R}\right)$.

Definition 1.1. Two $\mathbb{Z}$-lattices $L$ and $K$ are in the same genus if $L_{p} \cong K_{p}$ for all $p \in \mathbb{P} \cup\{\infty\}$.

It is well known that any genus consists of finitely many isometry classes (cf. Kne02, Satz (21.3)). We write $\mathcal{G}(L)$ for the set of all isometry classes in the genus of $L$ and define $h(L):=\# \mathcal{G}(L)$ to be the class number of $L$. A complete system of invariants for local isometry can be extracted from a decomposition of $L_{p}$ in modular sublattices.

TheOREM 1.2. Every integral $\mathbb{Z}$-lattice $L$ possesses a decomposition

$$
L_{p}=L_{0} \perp{ }^{p} L_{1} \perp \cdots \perp{ }^{p} L_{r},
$$

where all $L_{i}$ are unimodular (possibly zero-dimensional).

Proof. See ['M00], §91C.

In the literature, a splitting of the above form is called Jordan decomposition. We refer to $L$ as square-free if the Jordan decomposition of $L$ is of the form $L_{0} \perp{ }^{p} L_{1}$ at every prime $p$, and as strongly square-free if additionally $\operatorname{dim} L_{0} \geq \operatorname{dim} L_{1}$ holds. For $p \neq 2$ the Jordan decomposition is unique up to isometry. At the prime spot $p=2$ this is not true in general and only the following data remains invariant: $\operatorname{dim} L_{i}$, $2^{i}$ and the parity of every $L_{i}$. To address this problem, Conway and Sloane have introduced the notion of the so-called canonical Jordan decomposition. Among all splittings they have marked out one of a particular easy shape and have showed that it is uniquely determined for every $\mathbb{Z}_{2}$-lattice (cf. CS99, Chapter 15, 7.6). Before stating the main result of this section, we want to exemplify a somewhat technical detail. An interval of a Jordan decomposition is a finite sequence of (possibly zero-dimensional) consecutive modular components $\left({ }^{p^{i}} L_{i},{ }^{i+1} L_{i+1}, \ldots,{ }^{i+k} L_{i+k}\right)$. A compartment is a maximal interval in which all components are odd. Assume that the elements of a compartment are diagonalized over $\mathbb{Q}_{2}$ as $L_{i+j} \otimes_{\mathbb{Z}_{2}} \mathbb{Q}_{2} \cong$ $\left\langle a_{i+j, 1}, \ldots, a_{i+j, n}\right\rangle$. Then the oddity of a compartment is defined to be the sum of all traces modulo 8 , that is $\left(\sum_{l=1}^{n} a_{i, l}+\sum_{l=1}^{n} a_{i+1, l}+\ldots+\sum_{l=1}^{n} a_{i+k, l}\right) \bmod 8$. A very useful application is the following characterization of local isometry.

Theorem 1.3 (cf. CS99, Chapter 15, Theorem $9 \&$ 7.6). Let $L$ and $K$ be $\mathbb{Z}$-lattices and $(\dot{\bar{p}})$ the Jacobi symbol.

a) Let $p \neq 2$ and assume $L$ and $K$ are decomposed over $\mathbb{Z}_{p}$ as shown in theorem 1.2. Then $L_{p} \cong K_{p}$ if and only if $\left(\frac{\operatorname{det} L_{i}}{p}\right)=\left(\frac{\operatorname{det} K_{i}}{p}\right)$ and $\operatorname{dim} L_{i}=$ $\operatorname{dim} K_{i}$ for all $1 \leq i \leq r$. 
b) Let $L$ and $K$ be decomposed canonically over $\mathbb{Z}_{2}$. Then $L_{2} \cong K_{2}$ if and only if $\left(\frac{\operatorname{det} L_{i}}{2}\right)=\left(\frac{\operatorname{det} K_{i}}{2}\right), \operatorname{dim} L_{i}=\operatorname{dim} K_{i}$, the parity of $L_{i}$ is equal to the parity of $K_{i}$ for all $1 \leq i \leq r$ and the oddities of $L$ are equal to the oddities of $K$.

The invariants given by the Jordan decomposition are encoded in the genus symbol. A detailed introduction to this handy notation, in particular the quite technical realization for $p=2$, can be found in CS99, Chapter 15 and in Ber93. In the latter reference, all the missing proofs from [CS99 are carried out in detail. Here, we only mention that this symbol is a list of local symbols for each prime $p$ dividing $2 \operatorname{det} L$. Assuming a Jordan decomposition as above, the local symbol at the prime $p \neq 2$ is the formal product

$$
\prod_{i=0}^{r}\left(p^{i}\right)^{\varepsilon_{i}, n_{i}}, \text { where } \varepsilon_{i}:=\left(\frac{\operatorname{det} L_{i}}{p}\right) \text { and } n_{i}:=\operatorname{dim} L_{i} .
$$

In chapter 2 (resp. chapter 4), we will be using this notation for the enumeration of all primitive totally-reflective lattices in dimension 3 and 4 (resp. reflective lattices of signature $(5,1))$.

\subsection{The Mass Formula}

An indispensable tool for our investigation is the Minkowski-Siegel mass formula which relates the mass of a lattice to local quantities, which can be derived from the Jordan-decomposition and the invariants introduced in the previous section. Originally the mass formula is stated in terms of $p$-adic densities, cf. Sie35] for further details. However, for computational reasons, we find the approach of Conway and Sloane more suitable (cf. [CS88). Below we establish the main aspects.

Throughout this section we assume $L$ to be a positive-definite $\mathbb{Z}$-lattice. Hence $O(L)$ is a finite group and the following definition makes sense.

Definition 1.4. The mass of $L$ is defined as

$$
\mathrm{m}(L):=\sum_{M \in \mathcal{G}(L)} \frac{1}{|O(M)|} .
$$

It is a deep result from the analytic theory of quadratic forms that the mass of a lattice can be calculated with the local invariants introduced in the previous section (cf. theorem 1.3). In particular, the knowledge of the whole genus is not required. To amplify this, let $\Gamma$ denote the gamma function, $\zeta$ the Riemann zeta function and $\zeta_{D}$ the $L$-function

$$
\zeta_{D}(s)= \begin{cases}\prod_{p \in \mathbb{P}}\left(1-\left(\frac{D}{p}\right) \frac{1}{p^{s}}\right)^{-1}, & n \text { even, } \\ 1, & n \text { odd }\end{cases}
$$


where $n, D \in \mathbb{N}$ and $s:=\left\lceil\frac{n}{2}\right\rceil$. The fraction of the mass related to the odd prime numbers that do not divide the determinant is stored in the following quantity.

Definition 1.5. Let $n, D \in \mathbb{N}$ and $s:=\left\lceil\frac{n}{2}\right\rceil$. We refer to

$$
\operatorname{std}(n, D):=2 \pi^{-n(n+1) / 4} \cdot \prod_{j=1}^{n} \Gamma\left(\frac{j}{2}\right) \cdot \zeta(2) \zeta(4) \cdots \zeta(2 s-2) \zeta_{D}(s) .
$$

as the standard mass (with respect to $n$ and $D$ ).

As an example, we mention the dimensions we will be working with:

\begin{tabular}{|c|c|}
\hline$n$ & $\operatorname{std}(n, D)$ \\
\hline 2 & $\frac{2 \zeta_{D}(1)}{\pi}$ \\
3 & $\frac{1}{6}$ \\
4 & $\frac{\zeta_{D}(2)}{6 \pi^{2}}$ \\
\hline
\end{tabular}

TABLE 1. The standard mass in low dimensions.

Calculating $\zeta_{D}(s)$ in general requires numerous of techniques from analytic number theory (cf. CS88, section 8). It turns out that the behavior of $\zeta_{D}(s)$ as a function of $D$ is very erratic. The following lemma helps us to attain control.

Lemma 1.6. Let $D, s \in \mathbb{N}$ and $s \geq 2$. Then

a) $\zeta_{-D}(1) \leq 1+\frac{1}{2} \ln (D)$.

b) $\zeta_{D}(s) \geq \frac{\zeta(2 s)}{\zeta(s)}$.

Proof. Part a) goes back to Watson and can be found in Wat79, (5.10). To prove part b) the main idea is to use the Liouville function $\lambda(n):=(-1)^{\Omega(n)}$, where $\Omega(n)$ is the number of prime factors of $n$ counted with multiplicity, as a link between $\zeta(s)$ and $\zeta_{D}(s)$. We have

$$
\zeta_{D}(s)=\prod_{p \in \mathbb{P}}\left(1-\left(\frac{D}{p}\right) \frac{1}{p^{s}}\right)^{-1} \geq \prod_{p \in \mathbb{P}}\left(1+\frac{1}{p^{s}}\right)^{-1}=\sum_{n=1}^{\infty} \frac{\lambda(n)}{n^{s}},
$$

and the well-known identity $\frac{\zeta(2 s)}{\zeta(s)}=\sum_{n=1}^{\infty} \frac{\lambda(n)}{n^{s}}$ implies the assertion (cf. [Leh60]).

The actual mass of $L$ is gained from the standard mass by multiplying with certain correction factors, one for every prime $p$ dividing $2 \operatorname{det} L$. Unlike the standard mass, these correction factors depend on the local structure of $L$. 
Theorem 1.7. Let $L$ be an integral $\mathbb{Z}$-lattice with (canonized) Jordan decompositions $L_{p}=L_{0} \perp{ }^{p} L_{1} \perp \cdots \perp{ }^{r} L_{r}$ for every $p \in \mathbb{P}$. Let $n:=\operatorname{dim} L, s:=\left\lceil\frac{n}{2}\right\rceil, s_{i}:=$ $\left\lceil\frac{\operatorname{dim} L_{i}}{2}\right\rceil$ and $D:=(-1)^{s} \operatorname{det} L$. Then

$$
\mathrm{m}(L)=\operatorname{std}(n, D) \cdot \prod_{p \mid 2 \operatorname{det} L}\left(\mathrm{~m}_{\mathrm{p}}(L) \cdot 2 \prod_{j=2}^{s}\left(1-p^{2-2 j}\right)\right),
$$

where

$$
\mathrm{m}_{\mathrm{p}}(L)= \begin{cases}\prod_{i \in \mathbb{Z}} \mathrm{M}_{p}\left(L_{i}\right) \cdot \prod_{\substack{t, k \in \mathbb{Z}, k<t}} p^{\frac{1}{2}(t-k) \operatorname{dim} L_{t} \operatorname{dim} L_{k}}, & p \neq 2, \\ \prod_{i \in \mathbb{Z}} \mathrm{M}_{p}\left(L_{i}\right) \cdot \prod_{\substack{t, k \in \mathbb{Z}, k<t}} p^{\frac{1}{2}(t-k) \operatorname{dim} L_{t} \operatorname{dim} L_{k}} \cdot 2^{n(I, I)-n(I I)}, & p=2,\end{cases}
$$

with $n(I, I)$ being the total number of pairs of adjacent Jordan components $L_{i}, L_{i+1}$ that are both odd, $n(I I)$ the sum of the dimensions of all Jordan components that are even and

$$
\mathrm{M}_{p}\left(L_{i}\right):=\frac{1}{2}\left(1+\varepsilon p^{-s_{i}}\right)^{-1} \cdot \prod_{i=2}^{s_{i}}\left(1-p^{2-2 i}\right)^{-1} .
$$

The exact value of $\varepsilon \in\{0,1,-1\}$ depends on the species of the orthogonal group $O_{\operatorname{dim} L_{i}}\left(\mathbb{F}_{p}\right)$ associated with $L_{i}$ and can be found in [CS88, table 1 \& table 2.

Proof. See [CS88.

REMARK 1.8. Apart from a normalizing power of $p$, the quantity $\mathrm{M}_{p}\left(L_{i}\right)^{-1}$ is the order of the orthogonal group of a quadratic space over $\mathbb{F}_{p}$ associated with $L_{i}$. Which quadratic space has to be considered depends on the local structure of $L$. If $p$ is odd all that matters is $\left(\frac{\operatorname{det} L_{i}}{p}\right)$ and $\operatorname{dim} L_{i}$ and in the case $p=2$ only the oddity and parity must be taken into account additionally (cf. CS88, table 1 \& table 2). These are exactly the local invariants mentioned in theorem 1.3

With lemma 1.6 and the mass formula as stated in theorem 1.7, one can control the growth of the mass in the following sense.

Lemma 1.9. Let $L$ be an integral $\mathbb{Z}$-lattice.

a) If $\operatorname{dim} L=2$ and $L$ strongly square-free, then

$$
\mathrm{m}(L) \leq \frac{2}{\pi}\left(1+\frac{1}{2} \ln (\operatorname{det} L)\right) \cdot \frac{1}{2} \cdot \prod_{\substack{p \mid \operatorname{det} L, p \neq 2}} \frac{1}{2} \sqrt{p} .
$$

b) If $\operatorname{dim} L=2$ and $L$ square-free, then

$$
\mathrm{m}(L) \leq \frac{2}{\pi}\left(1+\frac{1}{2} \ln (\operatorname{det} L)\right) \cdot \frac{1}{2} \cdot \prod_{\substack{p \mid \operatorname{det} L, v_{p}(\operatorname{det} L)=2 \\ p \neq 2}} \frac{p}{p-1} \prod_{\substack{p \mid \operatorname{det} L, v_{p}(\operatorname{det} L)=1 \\ p \neq 2}} \frac{1}{2} \sqrt{p} .
$$


c) If $\operatorname{dim} L=3$ and $L$ strongly square-free, then

$$
\frac{1}{6} \cdot \frac{1}{8} \cdot \prod_{\substack{p \mid \operatorname{det} L, p \neq 2}} \frac{p-1}{2} \leq \mathrm{m}(L) .
$$

d) If $\operatorname{dim} L=4$ and $L$ strongly square-free, then

$$
\frac{1}{90} \cdot \frac{1}{24} \cdot \prod_{\substack{p \mid \operatorname{det} L, v_{p}(\operatorname{det} L)=2 \\ p \neq 2}} \frac{p^{2}(p-1)}{2 p+2} \prod_{\substack{p \mid \operatorname{det} L, v_{p}(\operatorname{det} L)=1 \\ p \neq 2}} \frac{1}{2} p^{\frac{3}{2}} \leq \mathrm{m}(L) .
$$

Proof. This follows directly from the mass formula 1.7 and lemma 1.6 .

\subsection{Positive-Definite Reflective Lattices}

Essential to the theory of hyperbolic reflection groups are reflective lattices. These are lattices that can be described very well by root systems (well-known objects of Lie theory). We recall the classification of positive-definite reflective lattices in low dimensions by Scharlau \& Blaschke (cf. [SB96]) and show that in these cases the root system already determines the whole isometry group of the lattice. This will be used in chapter 2 for bounding the prime factors of the determinant of totally-reflective genera.

Throughout this section we assume $(L, b)$ to be a positive-definite $\mathbb{Z}$-lattice. Each non-zero vector $v \in L$ gives rise to an Euclidean reflection of the enveloping vector space,

$$
s_{v}: V \longrightarrow V, \quad x \longmapsto x-\frac{2 b(x, v)}{b(v, v)} v .
$$

Each $s_{v}$ is an isometry of $V$. Reflective lattices are, from the geometrical point of view, those that possesses a high symmetry under such reflections. In order to measure the level of symmetry, we need a notion of root systems which is more general than the one usually used in the literature on Lie algebra theory.

Definition 1.10. Let $L$ be a positive-definite $\mathbb{Z}$-lattice.

a) A vector $v \in L$ is called root of $L$ if $v$ is primitive, that is $v / m \notin L$ for all integers $m>0$, and $s_{v}(L)=L$.

b) The set $R(L):=\{v \in L \mid v$ is a root of $L\}$ is called the root system of $L$.

c) The subgroup $W(L) \leqslant O(L)$ generated by all reflections $s_{v}$, with $v \in R(L)$, is called Weyl group of $L$.

It is easy to see that $R(L)$ is indeed a crystallographic root system (cf. [SB96, proposition 1.2). The root system of a lattice inherits the quadratic form, thus $R(L)$ decomposes into scaled irreducible components

$$
{ }^{\alpha} \mathrm{A}_{n},{ }^{\alpha} \mathrm{B}_{n},{ }^{\alpha} \mathrm{C}_{n},{ }^{\alpha} \mathrm{D}_{n},{ }^{\alpha} \mathrm{E}_{6},{ }^{\alpha} \mathrm{E}_{7},{ }^{\alpha} \mathrm{E}_{8},{ }^{\alpha} \mathrm{F}_{4},{ }^{\alpha} \mathrm{G}_{2} .
$$


Weyl groups are not affected by the scaling since a reflection $s_{v}$ does not depend on the length of $v$. The Dynkin diagram of such a "scaled" root system is the usual Dynkin diagram of the "unscaled" root system with the addition that every vertex is weighted with the scaling factor of the corresponding root. For example, the Dynkin diagram of ${ }^{5} \mathrm{~A}_{2}{ }^{7} \mathrm{E}_{5}$ is

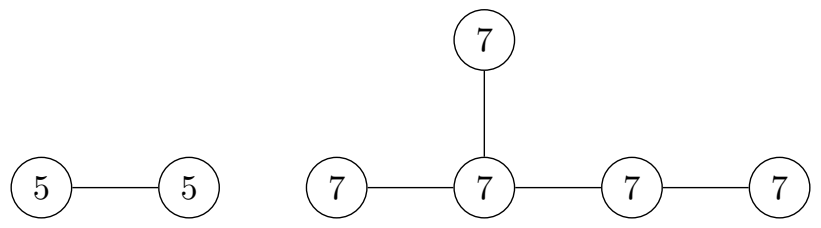

Unlike Weyl groups, the automorphism groups of Dynkin diagrams are affected by the scaling. Consider, for example, the root systems $A_{2} A_{2}$ and ${ }^{3} A_{2}{ }^{5} A_{2}$ with the corresponding diagrams
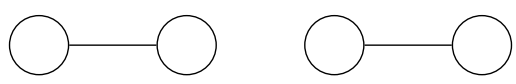

and
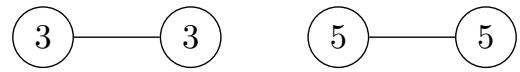

The automorphism group of the first one is the dihedral group $D i_{4}$ of order 8 , and $\mathbb{Z} / 2 \mathbb{Z} \times \mathbb{Z} / 2 \mathbb{Z}$ in the second case.

Let $R(L)$ be the root system of a lattice $L$ and $U:=K R(L)$ the subspace of $V$ generated by $R(L)$. The symmetry group of $R(L)$ is the stabilizer of $R(L)$ in $O(U)$ and will be denoted by $O(R(L))$. Every graph automorphism of the Dynkin diagram induces an element of the symmetry group of the root system, simply by permuting the roots in the same way the corresponding vertices are permuted in the diagram. One has $O(R(L)) \cong W(R(L)) \rtimes D(R(L))$ with $D(R(L))$ being the automorphism group of the Dynkin diagram of $R(L)$.

A positive-definite lattice is called reflective if, roughly spoken, its root system is large (and thus the level of symmetry under reflections high). More precisely:

Definition 1.11. A positive-definite lattice $L$ is called reflective if $R(L)$ generates a sublattice of the same rank.

As we will see in chapter 4, there is also a notion of reflectivity for indefinite lattices of signature $(n, 1)$. A useful characterization of reflectivity for positivedefinite lattices is given by the action of $W(L)$ on $V$.

Proposition 1.12. The lattice $L$ is reflective if and only if $W(L)$ has no nonzero fixed vectors. 
Proof. We write $\operatorname{Fix}(W(L))$ (resp. $\operatorname{Fix}\left(s_{v}\right)$ ) for the fixed vectors of $W(L)$ (resp. $s_{v} \in W(L)$ ). As we will see, both directions follow from the observation

$$
\operatorname{Fix}(W(L))=\bigcap_{v \in R(L)} \operatorname{Fix}\left(s_{v}\right)=\bigcap_{v \in R(L)} v^{\perp}=\left(\sum_{v \in R(L)} \mathbb{Q} v\right)^{\perp} .
$$

Thus, if $L$ is reflective then $\operatorname{Fix}(W(L))=\left(\sum_{v \in R(L)} \mathbb{Q} v\right)^{\perp}=V^{\perp}=\{0\}$. Conversely, the assumption $\{0\}=\operatorname{Fix}(W(L))$ implies $\left(\sum_{v \in R(L)} \mathbb{Q} v\right)^{\perp}=\{0\}=V^{\perp}$, which is equivalent to $\sum_{v \in R(L)} \mathbb{Q} v=V$, meaning $L$ is reflective.

The procedure in the next chapter is heavily based on the work of Scharlau \& Blaschke (cf. [SB96]). They classified all indecomposable reflective lattices in low dimensions by pairs $(R, \mathcal{L})$, where $R$ is a scaled root system and $\mathcal{L}$ the so-called glue code (a subgroup of the discriminant group of $\langle R\rangle$ ). Given a pair $(R, \mathcal{L})$, the associated lattice $L$ is constructed by $L=\langle\mathrm{R}\rangle+\langle x \mid \bar{x} \in \mathcal{L}\rangle$. The symmetries of $L$ are exactly the symmetries of $R$ which preserve the glue code: $O(L)=\{\varphi \in O(R) \mid \forall \bar{x} \epsilon$ $\mathcal{L}: \varphi(\bar{x}) \in \mathcal{L}\}$

THEOREM 1.13. Let $L$ be a reflective lattice.

a) If $\operatorname{dim} L=2$, then $L$ is isometric to one of the following:

\begin{tabular}{l|llll} 
& $\mathrm{R}$ & $\mathcal{L}$ & restrictions & determinant \\
\hline (a) & ${ }^{\alpha} \mathrm{A}_{1}{ }^{\beta} \mathrm{A}_{1}$ & $\mathcal{L}_{2,1}$ & $\begin{array}{l}\alpha<\beta \\
\alpha+\beta \equiv 0(2)\end{array}$ & $\alpha \beta$ \\
$(b)$ & ${ }^{\alpha} \mathrm{G}_{2}$ & 0 & $n o$ & $3 \alpha^{2}$ \\
$(c)$ & ${ }^{\alpha} \mathrm{B}_{1}{ }^{\beta} \mathrm{B}_{1}$ & 0 & $\alpha<\beta$ & $\alpha \beta$ \\
$(d)$ & ${ }^{\alpha} \mathrm{B}_{2}$ & 0 & no &
\end{tabular}

b) If $\operatorname{dim} L=3$ and $L$ is indecomposable, then $L$ is isometric to one of the following:

\begin{tabular}{l|llll} 
& $\mathrm{R}$ & $\mathcal{L}$ & restrictions & determinant \\
\hline (a) & ${ }^{\alpha} \mathrm{A}_{1}{ }^{\beta} \mathrm{A}_{1}{ }^{\gamma} \mathrm{A}_{1}$ & $\mathcal{L}_{3,1}$ & $\begin{array}{l}\alpha<\beta<\gamma \\
\alpha+\beta+\gamma \equiv 0(2)\end{array}$ & $2 \alpha \beta \gamma$ \\
(b) & ${ }^{\alpha} \mathrm{A}_{1}{ }^{\beta} \mathrm{A}_{1}{ }^{\gamma} \mathrm{A}_{1}$ & $\mathcal{L}_{3,2}$ & $\begin{array}{l}\alpha<\beta<\gamma \\
\alpha \equiv \beta \equiv \gamma \equiv 0(2)\end{array}$ & $\alpha \beta \gamma / 2$ \\
(c) & ${ }^{\alpha} \mathrm{A}_{1}{ }^{\beta} \mathrm{C}_{2}$ & $\mathcal{L}_{2,1}$ & $\begin{array}{l}\alpha \neq \beta, 2 \beta \\
\alpha \equiv 0(2)\end{array}$ & $2 \alpha \beta^{2}$ \\
(d) & ${ }^{\alpha} \mathrm{B}_{3}$ & $\neq 0$ & $\alpha \equiv 0(4)$ & $\alpha^{3} / 4$ \\
(e) & ${ }^{\alpha} \mathrm{C}_{3}$ & 0 & $n o$ & $4 \alpha^{3}$
\end{tabular}


c) If $\operatorname{dim} L=4$ and $L$ is indecomposable, then $L$ is isometric to one of the following:

\begin{tabular}{|c|c|c|c|c|}
\hline & $\mathrm{R}$ & $\mathcal{L}$ & restrictions & determinant \\
\hline$(a)$ & ${ }^{\alpha} \mathrm{A}_{1}{ }^{\beta} \mathrm{A}_{1}{ }^{\gamma} \mathrm{A}_{1}{ }^{\delta} \mathrm{A}_{1}$ & $\mathcal{L}_{4,1}$ & $\begin{array}{l}\alpha<\beta<\gamma<\delta \\
\alpha+\beta+\gamma+\delta \equiv 0(2)\end{array}$ & $4 \alpha \beta \gamma \delta$ \\
\hline$(b)$ & ${ }^{\alpha} \mathrm{A}_{1}{ }^{\beta} \mathrm{A}_{1}{ }^{\gamma} \mathrm{A}_{1}{ }^{\delta} \mathrm{A}_{1}$ & $\mathcal{L}_{4,2}$ & $\begin{array}{l}\alpha<\beta, \gamma<\delta \\
\alpha+\beta \equiv 0(2), \gamma \equiv \delta \equiv 0(2)\end{array}$ & $\alpha \beta \gamma \delta$ \\
\hline$(c)$ & ${ }^{\alpha} \mathrm{A}_{1}{ }^{\beta} \mathrm{A}_{1}{ }^{\gamma} \mathrm{A}_{1}{ }^{\delta} \mathrm{A}_{1}$ & $\mathcal{L}_{4,3}$ & $\begin{array}{l}\alpha<\beta<\gamma<\delta \\
\alpha \equiv \beta \equiv \gamma \equiv \delta \equiv 0(2)\end{array}$ & $\alpha \beta \gamma \delta / 4$ \\
\hline$(d)$ & ${ }^{\alpha} \mathrm{A}_{1}{ }^{\beta} \mathrm{A}_{1}{ }^{\gamma} \mathrm{C}_{2}$ & $\mathcal{L}_{3,1}$ & $\alpha<\beta, \alpha \neq \gamma \neq \beta$ & $4 \alpha \beta \gamma^{2}$ \\
\hline$(e)$ & ${ }^{\alpha} \mathrm{A}_{1}{ }^{\beta} \mathrm{A}_{1}{ }^{\gamma} \mathrm{C}_{2}$ & $\mathcal{L}_{3,2}$ & $\begin{array}{l}\alpha<\beta, \alpha \neq 2 \gamma \neq \beta \\
\alpha \equiv \beta \equiv 0(2)\end{array}$ & $\alpha \beta \gamma^{2}$ \\
\hline$(f)$ & ${ }^{\alpha} \mathrm{A}_{1}{ }^{\beta} \mathrm{B}_{3}$ & $\mathcal{L}_{2,1}$ & $2 \alpha \neq \beta, \beta \equiv 0(2)$ & $\alpha \beta^{3} / 2$ \\
\hline$(g)$ & ${ }^{\alpha} \mathrm{A}_{1}{ }^{\beta} \mathrm{C}_{3}$ & $\mathcal{L}_{2,1}$ & $\alpha \neq 2 \beta, \alpha \equiv 0(2)$ & $4 \alpha \beta^{3}$ \\
\hline$(h)$ & ${ }^{\alpha} \mathrm{A}_{2}{ }^{\beta} \mathrm{A}_{2}$ & $\mathcal{L}_{2,1}$ & $\begin{array}{l}\alpha<\beta, 2 \alpha \neq \beta \\
\alpha+\beta \equiv 0(3)\end{array}$ & $\alpha^{2} \beta^{2}$ \\
\hline (i) & ${ }^{\alpha} \mathrm{C}_{2}{ }^{\beta} \mathrm{C}_{2}$ & $\mathcal{L}_{2,1}$ & $\alpha<\beta$ & $4 \alpha^{2} \beta^{2}$ \\
\hline$(j)$ & ${ }^{\alpha} \mathrm{A}_{4}$ & 0 & no & $5 \alpha^{4}$ \\
\hline$(k)$ & ${ }^{\alpha} \mathrm{A}_{4}$ & $\neq 0$ & $\alpha \equiv 0(5)$ & $\alpha^{4} / 5$ \\
\hline$(l)$ & ${ }^{\alpha} \mathrm{F}_{4}$ & 0 & $\alpha \equiv 0(2)$ & $4 \alpha^{4}$ \\
\hline
\end{tabular}

Proof. See [SB96, proposition 4.4, theorem 4.5, theorem 4.7.

Of course, every lattice listed above is reflective. We refer to [SB96] for the precise definitions of the glue codes $\mathcal{L}_{i, j}$. As the first important application we will determine the isometry groups of reflective lattices in small dimensions. It turns out that $O(L)$ only depends on the combinatorial class of the root system of $L$.

Lemma 1.14. Let $L$ be a reflective lattice with $\operatorname{dim} L \in\{2,3,4\}$. Assume further that $L$ is indecomposable if $\operatorname{dim} L \in\{3,4\}$. Then $O(L)$ only depends on the combinatorial class of $R(L)$. In particular, $O(L)$ depends on neither the glue code nor the scaling. Referring to theorem 1.13 we have

a) in dimension 2 :

\begin{tabular}{c|c|c|c|c} 
& $(a)$ & $(b)$ & $(c)$ & $(d)$ \\
\hline$|O(L)|$ & 4 & 12 & 4 & 8
\end{tabular}


b) in dimension 3 :

\begin{tabular}{c|c|c|c|c|c} 
& $(a)$ & $(b)$ & $(c)$ & $(d)$ & $(e)$ \\
\hline$|O(L)|$ & 8 & 8 & 16 & 48 & 48
\end{tabular}

c) in dimension 4 :

\begin{tabular}{c|c|c|c|c|c|c} 
& $(a),(b),(c)$ & $(d),(e)$ & $(f),(g),(i)$ & $(h)$ & $(j),(k)$ & $(l)$ \\
\hline$|O(L)|$ & 16 & 32 & 96 & 72 & 240 & 1152
\end{tabular}

Proof. Let $R(L)$ be decomposed as $R(L)={ }^{\alpha_{1}} \mathrm{R}_{1} \cdots{ }^{\alpha_{k}} \mathrm{R}_{k}$ with $\mathrm{R}_{i}$ irreducible. The structure of the symmetry group $O(R(L)) \cong W(R(L)) \rtimes D(R(L))$, where $D(R(L))$ is the automorphism group of the Dynkin diagram of $R(L)$, and the reflectivity of $L$ imply the relation $W(R(L)) \subseteq O(L) \subseteq W(R(L)) \rtimes D(R(L))$.

All Dynkin diagrams that appear in dimension 2 and 3 (cf. theorem 1.13) have a trivial automorphism group, thus $O(L)=W(R(L))$. Furthermore, $W(R(L))$ does not depend on the scaling since $W\left({ }^{\alpha_{1}} \mathrm{R}_{1} \ldots{ }^{\alpha_{k}} \mathrm{R}_{k}\right) \cong W\left({ }^{\alpha_{1}} \mathrm{R}_{1}\right) \times \cdots \times W\left({ }^{\alpha_{k}} \mathrm{R}_{k}\right) \cong$ $W\left(\mathrm{R}_{1}\right) \times \cdots \times W\left(\mathrm{R}_{k}\right)$.

In dimension 4 , one can use the same argument, except in the cases $(j),(k)$ and $(h)$, where $D(R(L)) \neq\{$ id $\}$. There we have

$$
D(R(L))= \begin{cases}\mathbb{Z} / 2 \mathbb{Z}, & \text { in }(j) \text { and }(k), \\ \mathbb{Z} / 2 \mathbb{Z} \times \mathbb{Z} / 2 \mathbb{Z}, & \text { in }(h) .\end{cases}
$$

In both cases one easily checks the following: for $(j),(k)$, the non-trivial automorphism of the Dynkin diagram preserves both, the glue code of $(j)$ and $(k)$, thus $O(L)=W(R(L)) \rtimes \mathbb{Z} / 2 \mathbb{Z}$ depends on neither the gluce code nor the scaling. For $(h)$, only one of the three non-trivial automorphisms of the Dynkin diagram preserves the corresponding glue code, thus $O(L)=W(R(L)) \rtimes \mathbb{Z} / 2 \mathbb{Z}$ (cf. [SB96] for more details on the glue codes). Weyl groups of unscaled irreducible root systems, particularly their orders, are well known and can be found in Bou08.

Definition 1.15. Let $L$ be an integral lattice and $\mathcal{G}$ its genus.

a) We call $\mathcal{G}$ totally-reflective if each lattice in $\mathcal{G}$ is reflective.

b) The integral lattice $L$ is called totally-reflective if its genus $\mathcal{G}$ is totallyreflective.

We will see in chapter 4 that totally-reflective lattices play a major role in the understanding of hyperbolic reflection groups. Nevertheless, they are interesting objects of study in their own right. One can deduce from the work of Biermann Bie81 that there are only finitely many primitive totally-reflective genera in any dimension > 3 (cf. [SW92], Theorem 1.4). Furthermore, Esselmann proved in 
Ess96 that 20 is the largest dimension of totally-reflective genera, thus a classification is possible (at least) in principle. With the present work we contribute to this problem by classifying the dimensions 3 and 4 . 



\section{CHAPTER 2}

\section{Classification of Totally-Reflective Lattices}

\subsection{Bounds for the Determinant}

In this section we prove the results concerning step 1 and 2 of the general strategy described in the introduction. The primary goal is to find bounds for the prime factors of the determinant of totally-reflective lattices in dimension 3 and 4 . This will bring the number of local invariants, that need to be taken into account, to a manageable size and make the classification accessible for a computational approach. We will show that each prime factor of the determinant of a strongly square-free totally-reflective lattice in dimension 3 and 4 is bounded by a constant which only depends on the number of prime factors of the determinant. Furthermore, the number of prime factors is bounded by a constant which does not depend on the lattice at all. Bearing in mind that we are considering strongly square-free lattices, each prime factor can only appear with multiplicity 1 or 2 , thus the number of possible determinants (and hence the number of possible lattices) is finite.

In order to achieve these bounds, the basic idea is to compare the whole mass of a lattice $L$ with the part coming from the reflective lattices within $\mathcal{G}(L)$. Since the latter quantity is crucial, we make the following

Definition 2.1. Let $L$ be an integral lattice. We refer to

$$
\mathrm{m}_{\text {ref }}(L):=\sum_{\substack{M \in \mathcal{G}(L), M \text { is reflective }}} \frac{1}{|O(M)|}
$$

as the reflective part of the mass.

An important (though trivial) observation is that $\mathrm{m}_{\text {ref }}(L) \leq \mathrm{m}(L)$ and $L$ is totally-reflective iff $\mathrm{m}_{\text {ref }}(L)=\mathrm{m}(L)$. We obtain our bounds by showing that the reflective part of the mass grows more slowly than the whole mass (with increasing determinant). The growth of $\mathrm{m}(L)$ will be regulated by the mass formula which was introduced in the last chapter (cf. lemma 1.9). The behavior of $\mathrm{m}_{\text {ref }}(L)$ is controlled using the combinatorial classification of reflective lattices from SB96. (cf. theorem 1.13).

For this, it will be helpful to distinguish the lattices in $\mathcal{G}(L)$ by the type of the decomposition in indecomposable sublattices. For a 4-dimensional lattice $L$ we 
write

$$
\begin{aligned}
& \mathcal{G}_{4}(L)=\{M \in \mathcal{G}(L) \mid M \text { indecomposable }\}, \\
& \mathcal{G}_{3}(L)=\left\{M \in \mathcal{G}(L) \mid M=M_{1} \perp M_{2}, \operatorname{dim} M_{1}=3 \text { and } M_{1} \text { indecomposable }\right\}, \\
& \mathcal{G}_{2}(L)=\left\{M \in \mathcal{G}(L) \mid M=M_{1} \perp M_{2}, \operatorname{dim} M_{1}=2\right\} .
\end{aligned}
$$

Analogously, if $\operatorname{dim} L=3$ we define

$$
\begin{aligned}
& \mathcal{G}_{3}(L)=\{M \in \mathcal{G}(L) \mid M \text { indecomposable }\}, \\
& \mathcal{G}_{2}(L)=\left\{M \in \mathcal{G}(L) \mid M=M_{1} \perp M_{2}, \operatorname{dim} M_{1}=2\right\} .
\end{aligned}
$$

The reflective part of the mass can now be written as $\mathrm{m}_{\mathrm{ref}}(L)=\mathrm{m}_{\mathrm{ref} 4}(L)+\mathrm{m}_{\mathrm{ref} 3}(L)+$ $\mathrm{m}_{\mathrm{ref} 2}(L)$, with

$$
\mathrm{m}_{\text {ref } i}(L):=\sum_{\substack{M \in \mathcal{G}_{i}(L), M \text { reflective }}} \frac{1}{|O(M)|},
$$

$i=2,3,4$. Obviously, $\mathrm{m}_{\mathrm{ref} 4}$ is omitted when $\operatorname{dim} L=3$. Because we do not want to overload the notation, the dimension of the lattice we are dealing with is not included in the notation and will always be clear from the context. We write $\omega(d)$ (resp. $\Omega(d)$ ) for the number of (not necessarily) distinct prime factors of $d$. The divisor set of $d \in \mathbb{N}$ is $D(d):=\{x \in \mathbb{N} \mid x$ divides $d\}$ and $a(d):=\# D(d)$ is the number of distinct divisors of $d$.

LEMmA 2.2. Let $L$ be an integral $\mathbb{Z}$-lattice with determinant $d$.

a) For $\operatorname{dim} L=3$ we have

1) $\mathrm{m}_{\mathrm{ref} 3}(L) \leq \frac{17}{48} \cdot 3^{\Omega(d)}$,

2) $\mathrm{m}_{\mathrm{ref} 2}(L) \leq \frac{17}{48} \sum_{x \mid d} 2^{\Omega(x)}$.

b) For $\operatorname{dim} L=4$ we have

1) $\mathrm{m}_{\mathrm{ref} 4}(L) \leq \frac{611}{1920} \cdot 4^{\Omega(d)}$,

2) $\mathrm{m}_{\mathrm{ref} 3}(L) \leq \frac{17}{96} \sum_{x \mid d} 3^{\Omega(x)}$,

3) $\mathrm{m}_{\mathrm{ref} 2}(L) \leq \frac{17}{24} \cdot a(d) \cdot 2^{\Omega(d)}$.

Proof. We prove $b$ ) in detail to illustrate the basic idea. Part $a$ ) is proven analogously.

Estimating $\mathrm{m}_{\mathrm{ref} 4}(L)$ : First we give an upper bound for the number of possible isometry classes for every type (with type we mean the cases $(a)-(l)$ in theorem $1.13 \mathrm{c}$ )) of 4 -dimensional, indecomposable reflective lattices. To this end, it is sufficient to estimate how many scalings of $\mathrm{R}$ lead to the determinant $d$. According to theorem $1.13 \mathrm{c}$ ), the determinant $d$ of a lattice $L=\langle\mathrm{R}\rangle+\langle x \mid \bar{x} \in \mathcal{L}\rangle$ is a product $d=c \cdot \alpha_{1} \alpha_{2} \alpha_{3} \alpha_{4}$, where $\alpha_{i}$ is a scaling factor of $\mathrm{R}$ and $c \in\left\{1, \frac{1}{2}, \frac{1}{4}, \frac{1}{5}, 4,5\right\}$. The prime factors of $d$ can be distributed among the $\alpha_{i}$ in, at most, $4^{\Omega(d)}$ different ways (for simplicity, the restrictions for the scaling factors described in theorem 1.13 are 
ignored). Thus an upper bound for the number of isometry classes of reflective lattices of determinant $d$ which arise from $(\mathrm{R}, \mathcal{L})$ by changing the scaling of $\mathrm{R}$, is $4^{\Omega(d)}$. Combining this with lemma 1.14 , we get

$$
\mathrm{m}_{\text {ref } 4}(L) \leq 4^{\Omega(d)}\left(\frac{3}{16}+\frac{2}{32}+\frac{2}{72}+\frac{3}{96}+\frac{2}{240}+\frac{1}{1152}\right)=\frac{611}{1920} \cdot 4^{\Omega(d)} .
$$

Estimating $\mathrm{m}_{\mathrm{ref} 2}(L)$ : We use theorem $1.13 \mathrm{a}$ ) and the fact that $\left|O\left(M_{2}\right)\right| \geq 4$ for binary reflective lattices (cf. lemma 1.14): The same argumentation as in the case $\mathrm{m}_{\text {ref }}(L)$ shows that an upper bound for the number of isometry classes of binary reflective lattices of determinant $x$ is $4 \cdot 2^{\Omega(x)}$; there are 4 types of $(\mathrm{R}, \mathcal{L})$ and $2^{\Omega(x)}$ possibilities to change the scale of $\mathrm{R}$ in $(\mathrm{R}, \mathcal{L})$. Assuming a 4-dimensional reflective lattice decomposed as $M=M_{1} \perp M_{2}$, with $\operatorname{dim} M_{1}=2$ and $d=\operatorname{det} M, x=\operatorname{det} M_{1}$, each of the $4 \cdot 2^{\Omega(x)}$ isometry classes of $M_{1}$ can be combined with $4 \cdot 2^{\Omega(d / x)}$ isometry classes of $M_{2}$, leading to

$$
\begin{aligned}
\mathrm{m}_{\text {ref } 2}(L) & =\sum_{\substack{M \in \mathcal{G}_{2}(L), M \text { reflective }}} \frac{1}{|O(M)|} \\
& \leq \frac{1}{4} \sum_{x \mid d} 2^{\Omega(x)}\left(\frac{1}{4}+\frac{1}{12}+\frac{1}{4}+\frac{1}{8}\right) \cdot 4 \cdot 2^{\Omega(d / x)} \\
& =\frac{17}{24} \sum_{x \mid d} 2^{\Omega(d)} \\
& =\frac{17}{24} \cdot a(d) \cdot 2^{\Omega(d)} .
\end{aligned}
$$

Estimating $\mathrm{m}_{\mathrm{ref} 3}(L)$ : Assume a 4-dimensional reflective lattice decomposed as $M=M_{1} \perp M_{2}$ with $\operatorname{dim} M_{1}=3, M_{1}$ indecomposable and $d=\operatorname{det} M, x=\operatorname{det} M_{1}$. Unlike in the $\mathrm{m}_{\mathrm{ref} 2}$ case, the $4 \cdot 3^{\Omega(x)}$ isometry classes of $M_{1}$ can be combined with only one isometry class of $M_{2}$. This follows from the fact that $M_{1} \perp M_{2} \cong M_{1} \perp M_{2}^{\prime}$ implies $\operatorname{det} M_{2}=\operatorname{det} M_{2}^{\prime}$ and thus $M_{2} \cong M_{2}^{\prime}$, because of $\operatorname{dim} M_{2}=1=\operatorname{dim} M_{2}^{\prime}$. Hence

$$
\begin{aligned}
\mathrm{m}_{\text {ref } 3}(L) & =\sum_{\substack{M \in \mathcal{G}_{3}(L), \\
\text { reflective }}} \frac{1}{|O(M)|} \\
& \leq \frac{1}{2} \sum_{x \mid d} 2^{\Omega(x)}\left(\frac{1}{4}+\frac{1}{12}+\frac{1}{4}+\frac{1}{8}\right) \\
& =\frac{17}{48} \sum_{x \mid d} 2^{\Omega(d)} .
\end{aligned}
$$

For strongly square-free lattices the upper bound for $\mathrm{m}_{\mathrm{ref} 3}$ can be significantly sharpened (this was already known by Berger, cf. Ber93, Bemerkung 3.5.1).

Lemma 2.3. Let $L$ be a strongly square-free lattice of determinant d. Then, in both dimensions, we have $\mathrm{m}_{\mathrm{ref} 3}(L)=0$. 
Proof. Dimension 3: From theorem 1.13 it follows that 4 divides the determinant of a 3-dimensional, indecomposable reflective lattice, which is never the case for strongly square-free lattices.

Dimension 4: We show that a 4-dimensional reflective lattice cannot have a 3-dimensional, indecomposable orthogonal summand. Assume a reflective

$$
L=L^{\prime} \perp L^{\prime \prime},
$$

with $\operatorname{dim} L^{\prime}=3, \operatorname{dim} L^{\prime \prime}=1$ and $L^{\prime}$ indecomposable. theorem 1.13 b) implies $4 \mid \operatorname{det} L^{\prime}$, and $L$ being strongly square-free implies $8+\operatorname{det} L^{\prime}$. Notice that a lattice is even iff the unimodular component of the 2-adic Jordan decomposition is even (trivial), and that unimodular $\mathbb{Z}_{2}$-lattices only exist in even dimensions (cf. O'M00, 93:15). So if $L^{\prime}$ is even, then the 2-adic Jordan decomposition of $L^{\prime}$ is of the form $L^{\prime} \otimes \mathbb{Z}_{2}=L_{0}^{\prime} \perp{ }^{4} L_{2}^{\prime}$ with $\operatorname{dim} L_{0}^{\prime}=2$ and $\operatorname{dim} L_{2}^{\prime}=1$. This is a contradiction to $L$ being strongly square-free. If $L^{\prime}$ is odd, then theorem 1.13 implies that $L^{\prime}$ can only be of the shape $1.13 \mathrm{~b}),(a)$, which over $\mathbb{Z}$ always possesses a Gram matrix of the form $\left(\begin{array}{ccc}2 \alpha & 0 & \alpha \\ 0 & 2 \beta & \beta \\ \alpha & \beta & \delta\end{array}\right)$, with $\delta=\frac{\alpha+\beta+\gamma}{2}$, in some basis $v_{1}, v_{2}, v_{3} \in L$. Given that $L^{\prime}$ is odd, either $\alpha$ or $\beta$ is odd, so we may assume without loss of generality that $2+\beta$. Over $\mathbb{Z}_{2}$, the two vectors $v_{2}-\frac{\beta}{\delta} v_{3}, v_{3}$ ( $\delta$ is odd, thus a 2 -adic unit) generate an unimodular sublattice of $L^{\prime} \otimes \mathbb{Z}_{2}$ with Gram matrix $\left(\begin{array}{cc}2 \beta-\frac{\beta^{2}}{\delta} & 0 \\ 0 & \delta\end{array}\right)$; unimodular because $\beta$ is odd. As a unimodular sublattice, it is an orthogonal summand of $L^{\prime} \otimes \mathbb{Z}_{2}$ (cf. O’M00, 82:15), thus $L^{\prime} \otimes \mathbb{Z}_{2}$ must have a 1-dimensional 4-modular Jordan component. Again, this forms a contradiction to $L$ being strongly square-free. It follows $\mathrm{m}_{\mathrm{ref} 3}(L)=0$.

Let us make the following abbreviation for strongly square-free lattices $L$. If the dimension is 4 , we write

$$
\mathrm{M}_{\mathrm{ref} 4}(L)=\frac{611}{1920} \cdot 4^{\Omega(d)}, \quad \mathrm{M}_{\mathrm{ref} 2}(L)=\frac{17}{24} \cdot a(d) \cdot 2^{\Omega(d)},
$$

and

$$
\mathrm{M}_{\mathrm{ref}}(L)=\mathrm{M}_{\mathrm{ref} 4}(L)+\mathrm{M}_{\mathrm{ref} 2}(L)
$$

is an upper bound for $\mathrm{m}_{\mathrm{ref}}(L)$. The lower bound for the full mass from lemma 1.9 will be abbreviated as

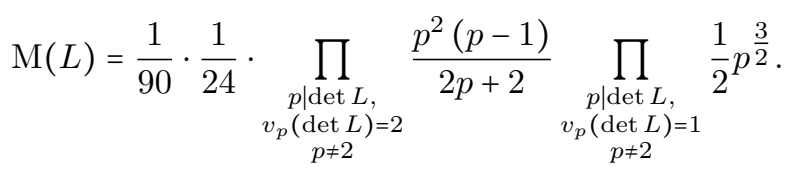

If the dimension is 3 , we write

$$
\mathrm{M}_{\mathrm{ref}}(L)=\mathrm{M}_{\mathrm{ref} 2}(L)=\frac{17}{48} \sum_{x \mid d} 2^{\Omega(x)}
$$


for the upper bound for $\mathrm{m}_{\mathrm{ref}}(L)$ and

$$
\mathrm{M}(L)=\frac{1}{6} \cdot \frac{1}{8} \cdot \prod_{\substack{p \mid \operatorname{det} L, p \neq 2}} \frac{p-1}{2}
$$

for the lower bound of $\mathrm{m}(L)$.

By combining lemma 1.9 and lemma 2.2 we see that a strongly square-free totally-reflective lattice satisfies the condition $\mathrm{M}_{\text {ref }}(L) / \mathrm{M}(L) \geq 1$. Actually, the estimates on $\mathrm{m}_{\text {ref }}(L)$ and $\mathrm{m}(L)$ depend only on the determinant of $L$, so we may write $\mathrm{M}(L)=\mathrm{M}(\operatorname{det} L)$ and $\mathrm{M}_{\text {ref }}(L)=\mathrm{M}_{\text {ref }}(\operatorname{det} L)$.

We now want to investigate how the ratio of $\mathrm{M}_{\mathrm{ref}}$ and $\mathrm{M}$ behaves when prime factors are appended to the determinant. Notice that both $\mathrm{M}_{\text {ref }}$ and $\mathrm{M}$ tend to $\infty$ when the number of prime factors increases.

Lemma 2.4. Let $d \in \mathbb{N}$ and $q \in \mathbb{P}$.

a) In dimension 3: For $q \geq 7$ and $q+d$ we have $\frac{\mathrm{M}_{\mathrm{ref}}(d)}{\mathrm{M}(d)} \geq \frac{\mathrm{M}_{\mathrm{ref}}(d q)}{\mathrm{M}(d q)}$.

b) In dimension 4: For $q \geq 7$ and $q+d$ we have $\frac{\mathrm{M}_{\mathrm{ref}}(d)}{\mathrm{M}(d)} \geq \frac{\mathrm{M}_{\mathrm{ref}}\left(d q^{2}\right)}{\mathrm{M}\left(d q^{2}\right)}$. For $q \geq 5$ and $q+d$ we have $\frac{\mathrm{M}_{\mathrm{ref}}(d)}{\mathrm{M}(d)} \geq \frac{\mathrm{M}_{\mathrm{ref}}(d q)}{\mathrm{M}(d q)}$.

Proof. We prove the first part of b) in detail so that the general idea is clear. The rest is proven analogously. From the mass formula it follows that

$$
\mathrm{M}\left(d q^{2}\right)=\frac{q^{2}(q-1)}{2(q+1)} \cdot M(d),
$$

thus the mass grows basically like a quadratic polynomial if the determinant is extended by a quadratic prime factor. The growth of $\mathrm{M}_{\mathrm{ref}}$ is much slower; there are constants $c_{1}$ and $c_{2}$ independent from $q$ (rather than a quadratic polynomial) such that

$$
\mathrm{M}_{\mathrm{ref} 4}\left(d q^{2}\right)=c_{1} \cdot \mathrm{M}_{\mathrm{ref} 4}(d), \quad \mathrm{M}_{\mathrm{ref} 2}\left(d q^{2}\right)=c_{2} \cdot \mathrm{M}_{\mathrm{ref} 2}(d) .
$$

The first statement follows immediately from the definition with $c_{1}:=4^{2}$. For the second statement, if taking into account that the divisors of $d q^{2}$ are the divisors of $d$ multiplied by $1, q$ and $q^{2}$, we get $a\left(d q^{2}\right)=3 \cdot a(d)$ and thus

$$
\begin{aligned}
\mathrm{M}_{\mathrm{ref} 2}\left(d q^{2}\right) & =\frac{17}{24} \cdot a\left(d q^{2}\right) \cdot 2^{\Omega\left(d q^{2}\right)}=\frac{17}{24} \cdot 3 \cdot a(d) \cdot 2^{2} \cdot 2^{\Omega(d)} \\
& =12 \cdot \mathrm{M}_{\mathrm{ref} 2}(d) .
\end{aligned}
$$

To prove the lemma, it is now sufficient to show that

$$
\begin{aligned}
& \frac{\mathrm{M}_{\mathrm{ref} 4}(d)}{\mathrm{M}(d)} \geq \frac{4^{2} \cdot \mathrm{M}_{\mathrm{ref} 4}(d)}{\mathrm{M}\left(d q^{2}\right)}, \\
& \frac{\mathrm{M}_{\mathrm{ref} 2}(d)}{\mathrm{M}(d)} \geq \frac{12 \cdot \mathrm{M}_{\mathrm{ref} 2}(d)}{\mathrm{M}\left(d q^{2}\right)} .
\end{aligned}
$$


Statement $(2.1)$ is equivalent to $\frac{q^{2}(q-1)}{2(q+1)} \geq 4^{2}$, and statement (2.2) is equivalent to $\frac{q^{2}(q-1)}{2(q+1)} \geq 12$. Both are true for $q \geq 7$.

The second lemma of this section clarifies the behavior of $M_{r e f} / M$ if the number of prime factors is fixed while the prime numbers increase. Again, a priori that is not clear since $M_{r e f}$ and $M$ tend to $\infty$ when the primes increase.

LEMMA 2.5. In both dimensions $\frac{\mathrm{M}_{\mathrm{ref}}(d)}{\mathrm{M}(d)}$ is monotonically decreasing in each prime factor of $d$.

Proof. The enumerator $\mathrm{M}_{\text {ref }}(d)$ only depends on the number of prime factors of $d$ and, as can be seen on the last two pages, the denominator $\mathrm{M}(d)$ is monotonically increasing in each prime factor.

The main theorem of this subsection is now a direct consequence of lemma 2.4 and lemma 2.5 .

THEOREM 2.6. Let $L$ be a strongly square-free totally-reflective lattice.

a) Let $\operatorname{dim} L=3$ and $\operatorname{det} L=q_{1} \cdots q_{s}$. Then $s \leq 9$.

b) Let $\operatorname{dim} L=4$ and $\operatorname{det} L=p_{1}^{2} \cdots p_{r}^{2} q_{1} \cdots q_{s}$. Then $r \leq 8$ and $s \leq 8-r$.

Proof. We have to decide when the necessary condition $\frac{\mathrm{M}_{\mathrm{ref}}}{\mathrm{M}} \geq 1$ is violated.

a) Let $L$ be a strongly square-free totally-reflective lattice with $\operatorname{det} L=q_{1} \cdots q_{s}$ and $s \geq 10$. Assume the prime factors are ordered such that $q_{1}<\cdots<q_{s}$. We start with the observation

$$
\frac{\mathrm{M}_{\mathrm{ref}}(2 \cdot 3 \cdot 5 \cdot 7 \cdot 11 \cdot 13 \cdot 17 \cdot 19 \cdot 23 \cdot 29)}{\mathrm{M}(2 \cdot 3 \cdot 5 \cdot 7 \cdot 11 \cdot 13 \cdot 17 \cdot 19 \cdot 23 \cdot 29)}<1 .
$$

Using the monotony statement of lemma 2.5 , we get

$$
\frac{\mathrm{M}_{\mathrm{ref}}\left(q_{1} \cdot q_{2} \cdot q_{3} \cdot q_{4} \cdot q_{5} \cdot q_{6} \cdot q_{7} \cdot q_{8} \cdot q_{9} \cdot q_{10}\right)}{\mathrm{M}\left(q_{1} \cdot q_{2} \cdot q_{3} \cdot q_{4} \cdot q_{5} \cdot q_{6} \cdot q_{7} \cdot q_{8} \cdot q_{9} \cdot q_{10}\right)}<1
$$

Now we apply lemma 2.4 a) and see that

$$
\frac{\mathrm{M}_{\mathrm{ref}}\left(q_{1} \cdots q_{10} \cdot q_{11} \cdots q_{s}\right)}{\mathrm{M}\left(q_{1} \cdots q_{10} \cdot q_{11} \cdots q_{s}\right)}=\frac{\mathrm{M}_{\mathrm{ref}}(\operatorname{det} L)}{\mathrm{M}(\operatorname{det} L)}<1 .
$$

Thus $L$ is not totally-reflective.

b) Let $L$ be a strongly square-free totally-reflective lattice of determinant $\operatorname{det} L=p_{1}^{2} \cdots p_{r}^{2} q_{1} \cdots q_{s}$ with $r \geq 9$. First we prove the statement regarding $r$. Assume $p_{1}<\cdots<p_{r}$ and $q_{1}<\cdots<q_{s}$. We have

$$
\frac{\mathrm{M}_{\mathrm{ref}}\left(2^{2} \cdot 3^{2} \cdot 5^{2} \cdot 7^{2} \cdot 11^{2} \cdot 13^{2} \cdot 17^{2} \cdot 19^{2} \cdot 23^{2}\right)}{\mathrm{M}\left(2^{2} \cdot 3^{2} \cdot 5^{2} \cdot 7^{2} \cdot 11^{2} \cdot 13^{2} \cdot 17^{2} \cdot 19^{2} \cdot 23^{2}\right)}<1
$$


and with lemma 2.5

$$
\frac{\mathrm{M}_{\mathrm{ref}}\left(p_{1}^{2} \cdot p_{2}^{2} \cdot p_{3}^{2} \cdot p_{4}^{2} \cdot p_{5}^{2} \cdot p_{6}^{2} \cdot p_{7}^{2} \cdot p_{8}^{2} \cdot p_{9}^{2}\right)}{\mathrm{M}\left(p_{1}^{2} \cdot p_{2}^{2} \cdot p_{3}^{2} \cdot p_{4}^{2} \cdot p_{5}^{2} \cdot p_{6}^{2} \cdot p_{7}^{2} \cdot p_{8}^{2} \cdot p_{9}^{2}\right)}<1
$$

Applying lemma $2.4 \mathrm{~b}$ ), we get

$$
\frac{\mathrm{M}_{\mathrm{ref}}\left(p_{1}^{2} \cdots p_{9}^{2} \cdot p_{10}^{2} \cdots p_{r}^{2} \cdot q_{1} \cdots q_{s}\right)}{\mathrm{M}\left(p_{1}^{2} \cdots p_{9}^{2} \cdot p_{10}^{2} \cdots p_{r}^{2} \cdot q_{1} \cdots q_{s}\right)}=\frac{\mathrm{M}_{\mathrm{ref}}(\operatorname{det} L)}{\mathrm{M}(\operatorname{det} L)}<1
$$

Thus $L$ is not totally-reflective.

To prove the statement concerning $s$, we fix the number of quadratic prime factors $r \leq 8$. Let $\operatorname{det} L=p_{1}^{2} \cdots p_{r}^{2} q_{1} \cdots q_{s}$ with $s \geq 9-r$. Define $\mathbb{P}(r+s)$ to be the finite set consisting of the first $r+s$ primes. For each combination $\left(\tilde{p}_{1}, \ldots, \tilde{p}_{r}, \tilde{q}_{1}, \ldots, \tilde{q}_{s}\right) \epsilon$ $\mathbb{P}(r+s)^{r+s}$ with $\tilde{p}_{1}<\cdots<\tilde{p}_{r}$ and $\tilde{q}_{1}<\cdots<\tilde{q}_{s}$ we have

$$
\frac{\mathrm{M}_{\mathrm{ref}}\left(\tilde{p}_{1}^{2} \cdots \tilde{p}_{r}^{2} \tilde{q}_{1} \cdots \tilde{p}_{s}\right)}{\mathrm{M}\left(\tilde{p}_{1}^{2} \cdots \tilde{p}_{r}^{2} \tilde{q}_{1} \cdots \tilde{p}_{s}\right)}<1 .
$$

Then lemma 2.4 and lemma 2.5 imply

$$
\frac{\mathrm{M}_{\mathrm{ref}}\left(p_{1}^{2} \cdots p_{r}^{2} q_{1} \cdots q_{s}\right)}{\mathrm{M}\left(p_{1}^{2} \cdots p_{r}^{2} q_{1} \cdots q_{s}\right)}<1
$$

In the next theorem we give upper bounds for the prime factors of the determinant. The tables below should be read as follows: $p_{1} \leq c_{1}$ means that the smallest prime factor of the determinant of a totally-reflective lattice is at most $c_{1}, p_{2} \leq c_{2}$ means that if the determinant has at least two prime factors then the second smallest is at most $c_{2}$, and so on. Thus, in dimension 4 , for example, $p_{7}$ is at most 449 regardless of whether there are 7,8 or 9 linear prime factors (and regardless how

\begin{tabular}{|c|c|c|c|c|c|c|c|c|c|}
\hline & $q_{1}$ & $q_{2}$ & $q_{3}$ & $q_{4}$ & $q_{5}$ & $q_{6}$ & $q_{7}$ & $q_{8}$ & $q_{9}$ \\
\hline$\leq$ & 103 & 307 & 919 & 1373 & 1373 & 827 & 409 & 151 & 47 \\
\hline
\end{tabular}
many quadratic prime factors there are).

THEOREM 2.7. Let $L$ be a strongly square-free totally-reflective lattice.

a) Let $\operatorname{dim} L=3$ and $\operatorname{det} L=q_{1} \cdots q_{s}$ with $s \leq 9$. Assume $q_{1}<\cdots<q_{s}$. Then

b) Let $\operatorname{dim} L=4$ and $\operatorname{det} L=p_{1}^{2} \cdots p_{r}^{2} q_{1} \cdots q_{s}$ with $r \leq 9$ and $s \leq 8-r$. Assume $p_{1}<\cdots<p_{r}$ and $q_{1}<\cdots<q_{s}$. Then

\begin{tabular}{c|c|c|c|c|c|c|c|c|c} 
& $p_{1}$ & $p_{2}$ & $p_{3}$ & $p_{4}$ & $p_{5}$ & $p_{6}$ & $p_{7}$ & $p_{8}$ & $p_{9}$ \\
\hline$\leq 191$ & 661 & 1601 & 2069 & 1831 & 997 & 449 & 157 & 47
\end{tabular}




\begin{tabular}{c|c|c|c|c|c|c|c|c} 
& $q_{1}$ & $q_{2}$ & $q_{3}$ & $q_{4}$ & $q_{5}$ & $q_{6}$ & $q_{7}$ & $q_{8}$ \\
\hline$\leq$ & 11287 & 6427 & 3613 & 1597 & 653 & 229 & 67 & 19
\end{tabular}

Proof. By using lemma 2.5 we can repeatedly increase a prime factor (and thus decrease the function $\mathrm{M}_{\mathrm{ref}} / \mathrm{M}$ ) until the necessary condition

$$
\mathrm{M}_{\text {ref }}(\operatorname{det} L) / \mathrm{M}(\operatorname{det} L) \geq 1
$$

is violated.

REMARK 2.8 .

a) With the help of theorem 2.6 and theorem 2.7, the enumeration of all strongly square-free totally-reflective genera can be carried out computationally. All calculations were performed using MAGMA, cf. [BCP97. First, we construct all genus symbols of strongly square-free lattices up to the given bounds. Avoiding redundant calculations by considering all the restrictions for genus symbols described in CS99, Chapter 15, this can be carried out in around 48 hours on the author's laptop computer. Then, to decide if a genus is totally-reflective, we enumerate all lattices within the genus, calculated their root system and checked whether it is of full rank. The non-trivial part here is the enumeration of the whole genus. Calculating the root system and its rank is a matter of milliseconds. The theory behind the enumeration of a genus is given by Kneser's neighborhood method, cf. Kne57. This method is implemented in MAGMA. There is also an implementation in C called TwoNeighbours (because the prime $p=2$ is used) going back to Scharlau \& Hemkemeier, cf. [SH98. The algorithm generates the whole genus if only one lattice from the genus is known. This one lattice that is required to start the neighborhood method (for a given genus symbol) is constructed according to LK13, section 5.2. Even in low dimension such as 3 and 4, enumerating a whole genus can be very time consuming if the determinant has large prime factors. Therefore, the neighborhood method has sometimes been interrupted after a couple of genus representatives have been found to check whether a nonreflective lattice has already occured. At the end a calculating time of 96 hours was necessary.

b) It turns out that the largest occuring value for the number of prime factors is

$$
(r, s)= \begin{cases}(3,3), & \text { in dimension } 4 \\ (0,4), & \text { in dimension } 3\end{cases}
$$


The largest prime factor $p$ occuring in dimension 4 is

$$
p= \begin{cases}13, & \text { if } v_{p}(\operatorname{det})=2, \\ 17, & \text { if } v_{p}(\operatorname{det})=1 .\end{cases}
$$

and $p=23$ in dimension 3 .

\subsection{Reduction to the Strongly Square-Free Case}

Now, after we have found all strongly square-free totally-reflective genera our next goal is to gradually weaken the restriction "strongly square-free". This is done in two steps. First, we drop the assumption "strong" by applying the partial dualization operator. In a second step we drop the assumption "square-free" by considering pre-images under the Watson transformation.

Definition 2.9. Let $L$ be a square-free lattice and $p \in \mathbb{P}$. The partial dual of $L$ at $p$ is defined as $\mathrm{D}_{p}(L):={ }^{p}\left(\frac{1}{p} L \cap L^{\#}\right)$.

In contrast to the usual dual operator, the partial dual operator only dualizes the lattice at the prime spot $p$. That means

$$
\mathrm{D}_{p}(L) \otimes_{\mathbb{Z}} \mathbb{Z}_{q}= \begin{cases}p\left(L_{q}^{\#}\right), & \text { if } q=p, \\ { }^{p} L_{q}, & \text { if } q \neq p .\end{cases}
$$

This has the following effect on the Jordan decomposition of a square-free lattice $L \otimes_{\mathbb{Z}} \mathbb{Z}_{p}=L_{0} \perp{ }^{p} L_{1}:$

$$
\mathrm{D}_{p}(L) \otimes_{\mathbb{Z}} \mathbb{Z}_{p} \cong L_{1} \perp{ }^{p} L_{0} .
$$

Thus, starting with a strongly square-free lattice, one can construct a (not necessarily strongly) square-free primitive lattice by applying $\mathrm{D}_{p}$ for $p \mid \operatorname{det} L$ (and vice versa). For a set of primes $I:=\left\{p_{1}, \cdots, p_{k}\right\} \subseteq \mathbb{P}$ we use the abbreviation $\mathrm{D}_{I}:=\mathrm{D}_{p_{1}} \circ \cdots \circ \mathrm{D}_{p_{k}}$ (where $\mathrm{D}_{\varnothing}:=\mathrm{id}$ ), which is well-defined since two partial dual operators with respect to different primes commute. Clearly, $\mathrm{D}_{p}$ extends to a welldefined bijective function $\mathcal{G}(L) \longrightarrow \mathrm{D}_{p}(\mathcal{G}(L))=\mathcal{G}\left(\mathrm{D}_{p}(L)\right)$. The next lemma shows that the partial dual behaves "well" relative to the property "totally-reflective".

Lemma 2.10. Let $L$ be an integral lattice. Then $L$ is totally-reflective if and only if $\mathrm{D}_{p}(L)$ is totally-reflective.

ProOF. Recall that the property "reflective" can be characterized by the action of $W(L)$ on $V$. Since $W(L)=W\left(\mathrm{D}_{p}(L)\right)$, it is clear that $L$ is reflective iff $\mathrm{D}_{p}(L)$ is reflective. Thus, the assertion follows from the bijectivity of $\mathrm{D}_{p}: \mathcal{G}(L) \longrightarrow$ $\mathrm{D}_{p}(\mathcal{G}(L))$. 
TheOREM 2.11. Let $\mathcal{T}_{n}$ be the set of all strongly square-free totally-reflective genera in dimension $n \in\{3,4\}$. Let $\mathcal{P}(d)$ be the power set of the set of all prime factors of $d:=\operatorname{det} \mathcal{G}$. Then

$$
\bigcup_{\mathcal{G} \in \mathcal{T}_{n}} \bigcup_{I \in \mathcal{P}(d)}\left\{\mathrm{D}_{I}(\mathcal{G})\right\}
$$

is the set of all square-free, primitive totally-reflective genera in dimension $n$.

Proof. This is a consequence of lemma 2.10, the bijectivity of $\mathcal{G}(L) \longrightarrow$ $\mathrm{D}_{p}(\mathcal{G}(L))$ and the above discussion.

The techniques we will use now are based on the following definition going back to Watson, cf. Wat62, Wat73.

Definition 2.12. Let $L$ be an integral lattice and $p \in \mathbb{P}$. The Watson transformation of $L$ at $p$ is defined as $\mathrm{E}_{p}(L):=L+\left(\frac{1}{p} L \cap p L^{\#}\right)$.

The usefulness of $\mathrm{E}_{p}$ becomes clear when we consider its effect on the Jordan decomposition. Let $L$ be an integral lattice with $L_{p}=L_{0} \perp{ }^{p} L_{1} \perp \cdots \perp{ }^{r} L_{r}$. Then

$$
\mathrm{E}_{p}(L) \otimes_{\mathbb{Z}} \mathbb{Z}_{q}= \begin{cases}\left(L_{0} \perp L_{2}\right) \perp{ }^{p}\left(L_{1} \perp L_{3}\right) \perp p^{2} L_{4} \perp \cdots \perp{ }^{r-2} L_{r}, & \text { if } q=p, \\ L \otimes_{\mathbb{Z}} \mathbb{Z}_{q}, & \text { if } q \neq p .\end{cases}
$$

Hence, after repeatedly applying the Watson transformation, a primitive lattice transforms into a square-free primitive lattice. Similar to the partial dual, $\mathrm{E}_{p}$ extends to a well-defined surjective function $\mathcal{G}(L) \longrightarrow \mathrm{E}_{p}(\mathcal{G}(L))=\mathcal{G}\left(\mathrm{E}_{p}(L)\right)$.

Lemma 2.13. If $L$ is totally-reflective lattice, then so is $\mathrm{E}_{p}(L)$.

Proof. The assertion implies that $W(L)$ has no non-zero fixed vectors, thus neither has $W\left(\mathrm{E}_{p}(L)\right)$ since $W(L) \subseteq W\left(\mathrm{E}_{p}(L)\right)$. Hence the assertion follows from the surjectivity of $\mathrm{E}_{p}$.

It may happen that prime factors disappear from the determinant after applying the Watson transformation. Thus, when calculating pre-images under $\mathrm{E}_{p}$, one has to decide which primes $p$ to consider (besides the prime factors of the determinant). An answer to this question is given by the following two lemmata.

Lemma 2.14. Let $L$ be an integral lattice, $p$ an odd prime with $p+\operatorname{det} L$ and $K \in \mathrm{E}_{p}(L)^{-1}$.

a) If $\operatorname{dim} L=3$ then,

$$
\mathrm{m}(K) \geq\left(\left(\frac{1}{1+p^{-1}}\right)^{2} p^{2}\left(1-p^{-2}\right)\right) \cdot \mathrm{m}(L) .
$$


b) If $\operatorname{dim} L=4$ then,

$$
\mathrm{m}(K) \geq\left(\frac{\zeta(4)}{2 \zeta(2)^{2}}\left(\frac{1}{1+p^{-1}}\right)^{2} p^{3}\left(1-p^{-2}\right)\right) \cdot \mathrm{m}(L) .
$$

Proof. This follows from the mass formula and lemma 1.6.

Lemma 2.15. Let $L$ be an integral lattice, $p$ an odd prime with $p+\operatorname{det} L$ and $K \in \mathrm{E}_{p}(L)^{-1}$.

a) If $\operatorname{dim} L=3$, then

$$
\mathrm{M}_{\mathrm{ref}}(K) \leq 81 \cdot \mathrm{M}_{\mathrm{ref}}(L) .
$$

b) If $\operatorname{dim} L=4$, then

$$
\mathrm{M}_{\mathrm{ref}}(K) \leq 4096 \cdot \mathrm{M}_{\mathrm{ref}}(L) .
$$

Proof. b) Since $L$ is not necessarily strongly square-free, we need to consider the upper bound for $\mathrm{m}_{\text {ref } 3}$ as well. Let $d:=\operatorname{det} L$. The assumption implies $K \otimes_{\mathbb{Z}} \mathbb{Z}_{p}=$ $K_{0} \perp p^{2} K_{2}$, in particular $\operatorname{det} K=\operatorname{det} L \cdot p^{2 n_{2}}$ where $n_{2}:=\operatorname{dim} K_{2} \in\{0,1,2,3\}$. Thus

$$
\mathrm{M}_{\mathrm{ref} 4}(K)=\frac{611}{1920} \cdot 4^{\Omega\left(d p^{2 n_{2}}\right)}=4^{2 n_{2}} \cdot \mathrm{M}_{\mathrm{ref} 4}(L) .
$$

For the other two cases we consider the decomposition of the divisor set

$$
D(\operatorname{det} K)=\bigcup_{i=0}^{2 n_{2}} p^{i} D(d) .
$$

Hence

$$
\mathrm{M}_{\mathrm{ref} 3}(K)=\sum_{i=0}^{2 n_{2}} \sum_{x \mid D(d)} \frac{17}{96} \cdot 3^{\Omega(x)} \cdot 3^{i}=\left(\frac{1-3^{2 n_{2}+1}}{1-3}\right) \cdot \mathrm{M}_{\mathrm{ref} 3}(L)
$$

and

$$
\mathrm{M}_{\mathrm{ref} 2}(K)=\frac{17}{24} \cdot a\left(d p^{2 n_{2}}\right) \cdot 2^{\Omega\left(d p^{2 n_{2}}\right)}=\left(2 n_{2}+1\right) \cdot 2^{n_{2}} \cdot \mathrm{M}_{\mathrm{ref} 2}(L) .
$$

Finally note that $\max \left\{4^{2 n_{2}},\left(\frac{1-3^{2 n_{2}+1}}{1-3}\right),\left(2 n_{2}+1\right) \cdot 2^{n_{2}}\right\} \leq 4096$ for $n_{2} \in\{1,2,3\}$.

Part a) of this lemma is proven analogously.

Corollary 2.16. Let $L$ be an integral lattice, $p$ an odd prime with $p+\operatorname{det} L$ and $K \in \mathrm{E}_{p}(L)^{-1}$.

a) If $K$ is totally-reflective and $\operatorname{dim} L=3$, then

$$
81 \cdot \frac{\mathrm{M}_{\mathrm{ref}}(L)}{\mathrm{m}(L)} \cdot\left(\left(\frac{1}{1+p^{-1}}\right)^{2} p^{2}\left(1-p^{-2}\right)\right)^{-1} \geq 1
$$

b) If $K$ is totally-reflective and $\operatorname{dim} L=4$, then

$$
4096 \cdot \frac{\mathrm{M}_{\mathrm{ref}}(L)}{\mathrm{m}(L)} \cdot\left(\frac{\zeta(4)}{2 \zeta(2)^{2}}\left(\frac{1}{1+p^{-1}}\right)^{2} p^{3}\left(1-p^{-2}\right)\right)^{-1} \geq 1 .
$$


Proof. Combine lemma 2.14 and lemma 2.15

Since the $p$-term in the above inequalities depends monotonically decreasingly on $p$ (for $p+2 \operatorname{det} L$ ) and $\mathrm{M}_{\text {ref }}(L) / \mathrm{m}(L)$ does not depend on $p$ at all, it is straightforward to decide when the statement of corollary 2.16 is satisfied.

\subsection{Classification Result}

Given the set of all square-free, primitive totally-reflective genera, one can produce all totally-reflective primitive genera by using corollary 2.16 and lemma 2.13 . First, corollary 2.16 tells us which (finitely many) primes one has to concider when calculating pre-images under $\mathrm{E}_{p}$. Then, during the process of repeatedly generating lattices $K \in \mathrm{E}_{p}(L)^{-1}$, lemma 2.13 tells us that we can stop and proceed with the next lattice when a not totally-reflective lattice occurs. Eventually this process will terminate since the number of totally-reflective genera is finite. Furthermore, lemma 2.13 also implies that every totally-reflective genus will be produced this way. We have implemented this procedure in MAGMA and it takes around 24 hours to find all totally-reflective genera.

THEOREM 2.17 .

a) In dimension 3 , there are 1234 primitive totally-reflective genera of which 289 are square-free and 52 strongly square-free.

b) In dimension 4, there are 930 primitive totally-reflective genera of which 230 are square-free and 88 strongly square-free.

To give some examples, we have listed below all totally-reflective genera which are strongly square-free. The complete list of all totally-reflective genera in dimension 3 and 4 is contained in the appendix.

\begin{tabular}{ccr|ccr|ccc} 
det & Genus & $\mathrm{h}$ & det & Genus & $\mathrm{h}$ & det & Genus & $\mathrm{h}$ \\
\hline & & & & & & & & \\
1 & $\mathrm{I}\left(1_{3}^{+3}\right)$ & 1 & 2 & $\mathrm{I}\left(2_{1}^{+1}\right)$ & 1 & 3 & $\mathrm{I}\left(3^{-1}\right)$ & 1 \\
3 & $\mathrm{I}\left(3^{+1}\right)$ & 1 & 5 & $\mathrm{I}\left(5^{-1}\right)$ & 1 & 5 & $\mathrm{I}\left(5^{+1}\right)$ & 1 \\
6 & $\mathrm{II}\left(2_{1}^{+1} 3^{-1}\right)$ & 1 & 6 & $\mathrm{I}\left(2_{1}^{+1} 3^{-1}\right)$ & 1 & 6 & $\mathrm{I}\left(2_{1}^{+1} 3^{+1}\right)$ & 1 \\
7 & $\mathrm{I}\left(7^{+1}\right)$ & 2 & 10 & $\mathrm{I}\left(2_{1}^{+1} 5^{-1}\right)$ & 2 & 10 & $\mathrm{I}\left(2_{1}^{+1} 5^{+1}\right)$ & 1 \\
11 & $\mathrm{I}\left(11^{-1}\right)$ & 1 & 14 & $\mathrm{II}\left(2_{1}^{+1} 7^{+1}\right)$ & 1 & 14 & $\mathrm{I}\left(2_{1}^{+1} 7^{+1}\right)$ & 2 \\
15 & $\mathrm{I}\left(3^{+1} 5^{+1}\right)$ & 2 & 15 & $\mathrm{I}\left(3^{-1} 5^{-1}\right)$ & 2 & 15 & $\mathrm{I}\left(3^{+1} 5^{-1}\right)$ & 1 \\
15 & $\mathrm{I}\left(3^{-1} 5^{+1}\right)$ & 1 & 17 & $\mathrm{I}\left(17^{+1}\right)$ & 2 & 21 & $\mathrm{I}\left(3^{+1} 7^{+1}\right)$ & 2 \\
21 & $\mathrm{I}\left(3^{-1} 7^{+1}\right)$ & 2 & 21 & $\mathrm{I}\left(3^{+1} 7^{-1}\right)$ & 1 & 30 & $\mathrm{II}\left(2_{1}^{+1} 3^{-1} 5^{-1}\right)$ & 2 \\
30 & $\mathrm{II}\left(2_{1}^{+1} 3^{+1} 5^{+1}\right)$ & 1 & 30 & $\mathrm{I}\left(2_{1}^{+1} 3^{-1} 5^{-1}\right)$ & 2 & 30 & $\mathrm{I}\left(2_{1}^{+1} 3^{+1} 5^{+1}\right)$ & 2 \\
30 & $\mathrm{I}\left(2_{1}^{+1} 3^{+1} 5^{-1}\right)$ & 1 & 33 & $\mathrm{I}\left(3^{+1} 11^{-1}\right)$ & 2 & 33 & $\mathrm{I}\left(3^{-1} 11^{+1}\right)$ & 2 \\
35 & $\mathrm{I}\left(5^{+1} 7^{-1}\right)$ & 1 & 35 & $\mathrm{I}\left(5^{-1} 7^{+1}\right)$ & 2 & 39 & $\mathrm{I}\left(3^{+1} 13^{-1}\right)$ & 1
\end{tabular}




\begin{tabular}{ccr|ccr|ccc}
42 & $\mathrm{II}\left(2_{7}^{+1} 3^{-1} 7^{+1}\right)$ & 2 & 42 & $\mathrm{I}\left(2_{1}^{+1} 3^{-1} 7^{-1}\right)$ & 2 & 42 & $\mathrm{I}\left(2_{1}^{+1} 3^{-1} 7^{+1}\right)$ & 2 \\
51 & $\mathrm{I}\left(3^{-1} 17^{+1}\right)$ & 2 & 57 & $\mathrm{I}\left(3^{+1} 19^{-1}\right)$ & 2 & 65 & $\mathrm{I}\left(5^{+1} 13^{+1}\right)$ & 2 \\
66 & $\mathrm{I}\left(2_{1}^{+1} 3^{+1} 11^{-1}\right)$ & 3 & 69 & $\mathrm{I}\left(3^{-1} 23^{+1}\right)$ & 3 & 77 & $\mathrm{I}\left(7^{+1} 11^{-1}\right)$ & 3 \\
78 & $\mathrm{II}\left(2_{1}^{+1} 3^{-1} 13^{-1}\right)$ & 3 & 105 & $\mathrm{I}\left(3^{+1} 5^{-1} 7^{+1}\right)$ & 2 & 105 & $\mathrm{I}\left(3^{-1} 5^{-1} 7^{-1}\right)$ & 2 \\
105 & $\mathrm{I}\left(3^{-1} 5^{+1} 7^{+1}\right)$ & 2 & 105 & $\mathrm{I}\left(3^{-1} 5^{-1} 7^{+1}\right)$ & 3 & 165 & $\mathrm{I}\left(3^{+1} 5^{+1} 11^{-1}\right)$ & 2 \\
195 & $\mathrm{I}\left(3^{-1} 5^{-1} 13^{-1}\right)$ & 3 & 210 & $\mathrm{II}\left(2_{7}^{+1} 3^{+1} 5^{+1} 7^{+1}\right)$ & 2 & 210 & $\mathrm{I}\left(2_{1}^{+1} 3^{+1} 5^{+1} 7^{+1}\right)$ & 3 \\
330 & $\mathrm{II}\left(2_{7}^{+1} 3^{-1} 5^{-1} 11^{-1}\right)$ & 4 & & & & &
\end{tabular}

TABLE 1. Strongly square-free totally-reflective genera in dimension 3.

\begin{tabular}{|c|c|c|c|c|c|c|c|c|}
\hline $\operatorname{det}$ & Genus & $\mathrm{h}$ & $\operatorname{det}$ & Genus & $\mathrm{h}$ & $\operatorname{det}$ & Genus & h \\
\hline 1 & $\mathrm{I}\left(1_{4}^{+4}\right)$ & 1 & 2 & $\mathrm{I}\left(2_{1}^{+1}\right)$ & 1 & 3 & $\mathrm{I}\left(3^{-1}\right)$ & 1 \\
\hline 3 & $\mathrm{I}\left(3^{+1}\right)$ & 1 & 4 & $\mathrm{II}\left(2_{\mathrm{II}}^{-2}\right)$ & 1 & 4 & $\mathrm{I}\left(2_{2}^{+2}\right)$ & 1 \\
\hline 5 & $\mathrm{II}\left(5^{+1}\right)$ & 1 & 5 & $\mathrm{I}\left(5^{+1}\right)$ & 1 & 5 & $\mathrm{I}\left(5^{-1}\right)$ & 1 \\
\hline 6 & $\mathrm{I}\left(2_{1}^{+1} 3^{-1}\right)$ & 2 & 6 & $\mathrm{I}\left(2_{1}^{+1} 3^{+1}\right)$ & 1 & 7 & $\mathrm{I}\left(7^{+1}\right)$ & 2 \\
\hline 9 & $\mathrm{II}\left(3^{+2}\right)$ & 1 & 9 & $\mathrm{I}\left(3^{+2}\right)$ & 1 & 9 & $\mathrm{I}\left(3^{-2}\right)$ & 1 \\
\hline 10 & $\mathrm{I}\left(2_{1}^{+1} 5^{-1}\right)$ & 2 & 12 & $\mathrm{II}\left(2_{2}^{+2} 3^{-1}\right)$ & 1 & 12 & $\mathrm{II}\left(2_{6}^{+2} 3^{+1}\right)$ & 1 \\
\hline 12 & $\mathrm{I}\left(2_{2}^{+2} 3^{-1}\right)$ & 1 & 12 & $\mathrm{I}\left(2_{2}^{+2} 3^{+1}\right)$ & 1 & 14 & $\mathrm{I}\left(2_{1}^{+1} 7^{+1}\right)$ & 3 \\
\hline 15 & $\mathrm{I}\left(3^{+1} 5^{+1}\right)$ & 2 & 15 & $\mathrm{I}\left(3^{-1} 5^{-1}\right)$ & 3 & 15 & $\mathrm{I}\left(3^{+1} 5^{-1}\right)$ & 1 \\
\hline 18 & $\mathrm{I}\left(2_{1}^{+1} 3^{-2}\right)$ & 2 & 18 & $\mathrm{I}\left(2_{1}^{+1} 3^{+2}\right)$ & 2 & 20 & $\mathrm{II}\left(2_{\mathrm{II}}^{+2} 5^{+1}\right)$ & 1 \\
\hline 20 & $\mathrm{I}\left(2_{2}^{+2} 5^{-1}\right)$ & 2 & 20 & $\mathrm{I}\left(2_{2}^{+2} 5^{+1}\right)$ & 2 & 20 & $\mathrm{II}\left(2_{\mathrm{II}}^{-2} 5^{-1}\right)$ & 1 \\
\hline 21 & $\operatorname{II}\left(3^{-1} 7^{+1}\right)$ & 1 & 21 & $\mathrm{I}\left(3^{-1} 7^{+1}\right)$ & 2 & 25 & $\operatorname{II}\left(5^{-2}\right)$ & 1 \\
\hline 25 & $\mathrm{I}\left(5^{-2}\right)$ & 1 & 25 & $\mathrm{I}\left(5^{+2}\right)$ & 2 & 28 & $\mathrm{II}\left(2_{2}^{+2} 7^{+1}\right)$ & 2 \\
\hline 30 & $\mathrm{I}\left(2_{1}^{+1} 3^{-1} 5^{-1}\right)$ & 4 & 33 & $\mathrm{I}\left(3^{+1} 11^{-1}\right)$ & 2 & 36 & $\mathrm{II}\left(2_{\mathrm{II}}^{-2} 3^{-2}\right)$ & 1 \\
\hline 36 & $\mathrm{II}\left(2_{\mathrm{II}}^{+2} 3^{+2}\right)$ & 1 & 36 & $\mathrm{II}\left(2_{0}^{+2} 3^{+2}\right)$ & 1 & 36 & $\mathrm{I}\left(2_{2}^{+2} 3^{-2}\right)$ & 2 \\
\hline 36 & $\mathrm{I}\left(2_{2}^{+2} 3^{+2}\right)$ & 2 & 45 & $\mathrm{II}\left(3^{+2} 5^{-1}\right)$ & 1 & 45 & $\mathrm{II}\left(3^{-2} 5^{+1}\right)$ & 1 \\
\hline 45 & $\mathrm{I}\left(3^{+2} 5^{-1}\right)$ & 2 & 45 & $\mathrm{I}\left(3^{-2} 5^{+1}\right)$ & 2 & 49 & $\operatorname{II}\left(7^{+2}\right)$ & 1 \\
\hline 60 & $\mathrm{II}\left(2_{2}^{+2} 3^{+1} 5^{+1}\right)$ & 2 & 60 & $\mathrm{I}\left(2_{2}^{+2} 3^{-1} 5^{-1}\right)$ & 3 & 60 & $\mathrm{II}\left(2_{6}^{+2} 3^{+1} 5^{-1}\right)$ & 1 \\
\hline 63 & $\mathrm{I}\left(3^{+2} 7^{-1}\right)$ & 3 & 63 & $\mathrm{I}\left(3^{+2} 7^{+1}\right)$ & 3 & 68 & $\mathrm{II}\left(2_{\mathrm{II}}^{-2} 17^{+1}\right)$ & 2 \\
\hline 75 & $\mathrm{I}\left(3^{-1} 5^{+2}\right)$ & 3 & 75 & $\mathrm{I}\left(3^{+1} 5^{-2}\right)$ & 3 & 84 & $\operatorname{II}\left(2_{\mathrm{II}}^{+2} 3^{-1} 7^{+1}\right)$ & 2 \\
\hline 84 & $\mathrm{II}\left(2_{\mathrm{II}}^{-2} 3^{+1} 7^{+1}\right)$ & 2 & 90 & $\mathrm{I}\left(2_{1}^{+1} 3^{+2} 5^{+1}\right)$ & 4 & 90 & $\mathrm{I}\left(2_{1}^{+1} 3^{+2} 5^{-1}\right)$ & 4 \\
\hline 98 & $\mathrm{I}\left(2_{1}^{+1} 7^{+2}\right)$ & 4 & 100 & $\mathrm{II}\left(2_{\mathrm{II}}^{-2} 5^{+2}\right)$ & 1 & 100 & $\mathrm{II}\left(2_{\mathrm{II}}^{+2} 5^{-2}\right)$ & 1 \\
\hline 100 & $\mathrm{I}\left(2_{2}^{+2} 5^{-2}\right)$ & 2 & 117 & $\mathrm{II}\left(3^{+2} 13^{-1}\right)$ & 2 & 132 & $\mathrm{II}\left(2_{\mathrm{II}}^{-2} 3^{+1} 11^{-1}\right)$ & 2 \\
\hline 132 & $\mathrm{II}\left(2_{\mathrm{II}}^{-2} 3^{-1} 11^{+1}\right)$ & 2 & 180 & $\mathrm{II}\left(2_{\mathrm{II}}^{+2} 3^{+2} 5^{-1}\right)$ & 2 & 180 & $\operatorname{II}\left(2_{\mathrm{II}}^{+2} 3^{-2} 5^{+1}\right)$ & 2 \\
\hline 180 & $\mathrm{II}\left(2_{\mathrm{II}}^{-2} 3^{+2} 5^{+1}\right)$ & 2 & 180 & $\mathrm{II}\left(2_{\mathrm{II}}^{-2} 3^{-2} 5^{-1}\right)$ & 2 & 196 & $\mathrm{II}\left(2_{\mathrm{II}}^{-2} 7^{-2}\right)$ & 2 \\
\hline 225 & $\mathrm{II}\left(3^{+2} 5^{+2}\right)$ & 2 & 225 & $\mathrm{I}\left(3^{+2} 5^{-2}\right)$ & 2 & 252 & $\mathrm{II}\left(2_{2}^{+2} 3^{+2} 7^{-1}\right)$ & 3 \\
\hline 252 & $\mathrm{II}\left(2_{6}^{+2} 3^{+2} 7^{+1}\right)$ & 3 & 300 & $\mathrm{II}\left(2_{2}^{+2} 3^{-1} 5^{+2}\right)$ & 3 & 300 & $\mathrm{II}\left(2_{2}^{+2} 3^{+1} 5^{-2}\right)$ & 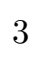 \\
\hline
\end{tabular}




\begin{tabular}{ccc|ccc|ccc}
420 & $\mathrm{II}\left(2_{\mathrm{II}}^{-2} 3^{+1} 5^{-1} 7^{+1}\right)$ & 3 & 420 & $\mathrm{II}\left(2_{\mathrm{II}}^{-2} 3^{-1} 5^{+1} 7^{+1}\right)$ & 3 & 441 & $\mathrm{I}\left(3^{+2} 7^{+2}\right)$ & 4 \\
484 & $\mathrm{II}\left(2_{\mathrm{II}}^{-2} 11^{-2}\right)$ & 1 & 900 & $\mathrm{II}\left(2_{\mathrm{II}}^{-2} 3^{-2} 5^{+2}\right)$ & 2 & 900 & $\mathrm{II}\left(2_{\mathrm{II}}^{-2} 3^{+2} 5^{-2}\right)$ & 2 \\
900 & $\mathrm{I}\left(2_{2}^{+2} 3^{+2} 5^{-2}\right)$ & 4 & 1764 & $\mathrm{II}\left(2_{\mathrm{II}}^{-2} 3^{+2} 7^{+2}\right)$ & 2 & 4900 & $\mathrm{II}\left(2_{\mathrm{II}}^{-2} 5^{-2} 7^{+2}\right)$ & 2 \\
6084 & $\mathrm{II}\left(2_{\mathrm{II}}^{-2} 3^{+2} 13^{-2}\right)$ & 2 & & & & & &
\end{tabular}

TABLE 2. Strongly square-free totally-reflective genera in dimension 4 . 


\section{CHAPTER 3}

\section{Hyperbolic Reflection Groups}

\subsection{Fundamentals on Hyperbolic Reflection Groups}

Throughout this chapter let $(V,()$,$) be a Lorentzian space of signature (n, 1)$. The set

$$
F^{n}:=\{v \in V \mid(v, v)=-1\}
$$

falls into two connected components (see figure 1). We pick an arbitrary component $H^{n}$ and stick with it from now on. For two points $x, y \in H^{n}$ there is a unique nonnegative real number $\rho(x, y)$ such that

$$
(x, y)=\|x\|\|y\| \cosh \rho(x, y),
$$

cf. Rat06, 3.1.7. This number is called the hyperbolic distance between $x$ and $y$. The mapping

$$
\rho: H^{n} \times H^{n} \longrightarrow \mathbb{R},(x, y) \longmapsto \rho(x, y)
$$

is a metric and the metric space $\left(H^{n}, \rho\right)$ is a model for the hyperbolic $n$-space, cf. Rat06, 3.2.2.

The geometry of $H^{n}$ is closely related to the linear structure of $V$. Every isometry of $H^{n}$ (i.e. map preserving the metric $\rho$ ) can be written as the restriction

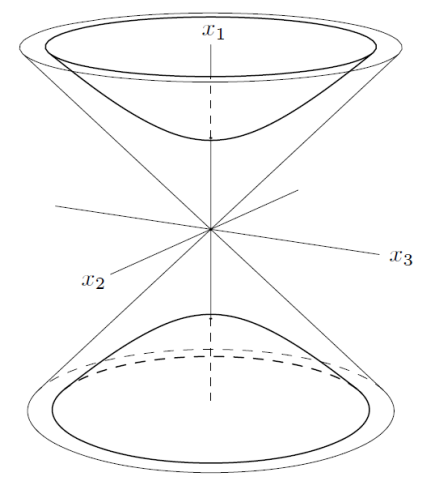

Figure 1. The two-sheeted hyperboloid $F^{n}$ inside the light cone consisting of all negative-norm vectors (image taken from Rat06]. 


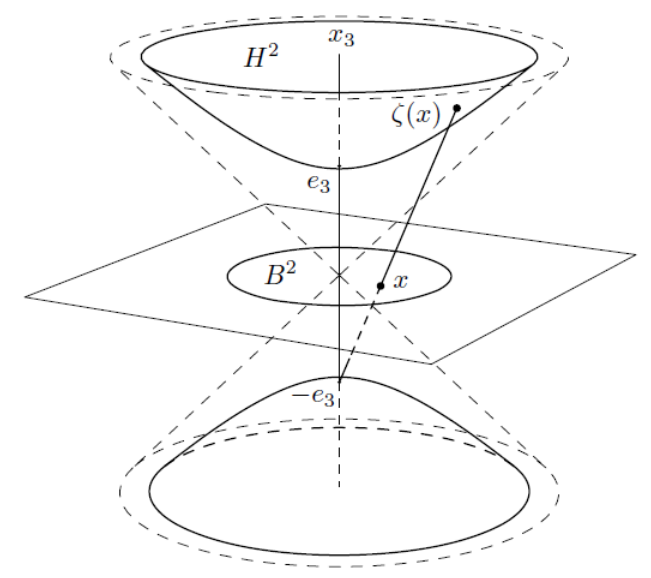

Figure 2. The stereographic projection $\zeta$ of $B^{2}$ onto $H^{2}$ (image taken from [Rat06]).

of a linear isometry of $V$ to $H^{n}$. Conversely, every linear isometry of $V$ that maps $H^{n}$ onto itself induces (by restriction) an isometry of $\left(H^{n}, \rho\right)$, cf. [Rat06, 3.2.3. In this sense, the full isometry group of $H^{n}$ can be found within $O(V)$ : it is the subgroup $O^{+}(V)$ of index 2 consisting of those isometries of $V$ mapping each of the connected components onto itself.

A hyperplane $h_{v}$ of $H^{n}$ is by definition the intersection of $H^{n}$ with an indefinite linear hyperplane of $V$,

$$
h_{v}:=H^{n} \cap v^{\perp} \quad \text { with } \quad v \in V,(v, v)>0 .
$$

Let $h_{v}$ be a hyperplane with the corresponding linear subspace $v^{\perp}$. The linear reflection in $v^{\perp}$

$$
s_{v}: V \longrightarrow V, x \longmapsto x-\frac{2(x, v)}{(v, v)} v
$$

is an isometry of $V$ and the corresponding isometry $\sigma_{v}:=s_{v} \mid H^{n}$ of $\left(H^{n}, \rho\right)$ is called the hyperbolic reflection in $h_{v}$.

Definition 3.1. A discrete subgroup of $O^{+}(V)$ generated by reflections is called a hyperbolic reflection group.

When working in the hyperbolic 2-space it is very convenient to use the conformal ball model $B^{2}$ of the hyperbolic plane. We do not give a detailed description and only mention that this model is constructed by identifying each point in $H^{2}$ with a point in the open unit circle via the stereographic projection (cf. figure 2).

A hyperbolic reflection group acts naturally on $H^{n}$ and it is helpful to consider the fundamental domain of this action. 


\section{DEFINITION 3.2.}

a) Let $h_{v}$ be a hyperbolic hyperplane. The set $h_{v}^{+}:=\left\{x \in H^{n} \mid(x, v) \geq 0\right\}$ is called the closed half-space bounded by $h_{v}$.

b) A polyhedron $P$ is the intersection of a locally finite system of closed halfspaces containing a non-empty open set,

$$
P:=\bigcap_{i \in I} h_{v_{i}}^{+} .
$$

c) Let $P$ be a polyhedron. We say $P$ is a Coxeter polyhedron if all dihedral angles of $P$ are of the form $\frac{\pi}{m}$ with $m \in\{2,3, \ldots\}$.

For the definition of the dihedral angle and further notions (such as faces, cusps) related to geometry of polyhedra we refer to Rat06, $\S 6.3, \S 6.4$.

\section{Proposition 3.3.}

a) The fundamental domain of a hyperbolic reflection group acting on $H^{n}$ is a Coxeter polyhedron.

b) Every Coxeter polyhedron is the fundamental domain of a hyperbolic reflection group.

Proof. Part a) can be found in Rat06, Theorem 7.1.2. To prove part b), consider the group generated by the reflections with respect to the faces of the given Coxeter polyhedron. A detailed proof can be found in Rat06, Theorem 7.13.

EXAMPLE 3.4. Let $\Delta$ be a simplex in the hyperbolic 2 -space, i.e. a compact polyhedron with exactly 3 vertices. Assume further that $\Delta$ is a Coxeter polyhedron. Thus there are integers $a, b, c \in \mathbb{Z}$ with $2 \leq a \leq b \leq c$ such that $\Delta=T(a, b, c)$ is the triangle whose angles are $\frac{\pi}{a}, \frac{\pi}{b}, \frac{\pi}{c}$. The hyperbolic reflection group generated by the reflections in the sides of $T(a, b, c)$ is denoted by $W(a, b, c)$. In hyperbolic geometry the sum of the angles of a triangle is less than $\pi$ (cf. Rat06, Theorem 3.5.1),

$$
\frac{\pi}{a}+\frac{\pi}{b}+\frac{\pi}{c}<\pi
$$

thus the integers $a, b, c$ satisfy the inequality

$$
\frac{1}{a}+\frac{1}{b}+\frac{1}{c}<1
$$

which has an infinite number of solutions. Each solution induces a triangle $T(a, b, c)$ and a corresponding hyperbolic reflection group $W(a, b, c)$. The action of the group $W(2,4,6)$ on $B^{2}$ is visualized in figure 3 A classification for these types of reflection groups is known and accessible by relatively elementary methods.

Theorem (cf. Rat06, Theorem 7.2.1). Let $a, b, c, a^{\prime}, b^{\prime}, c^{\prime}$, be integers such that $2 \leq a \leq b \leq c$ and $2 \leq a^{\prime} \leq b^{\prime} \leq c^{\prime}$. Then $W(a, b, c)$ is isomorphic to $W\left(a^{\prime}, b^{\prime}, c^{\prime}\right)$ iff $(a, b, c)=\left(a^{\prime}, b^{\prime}, c^{\prime}\right)$. 


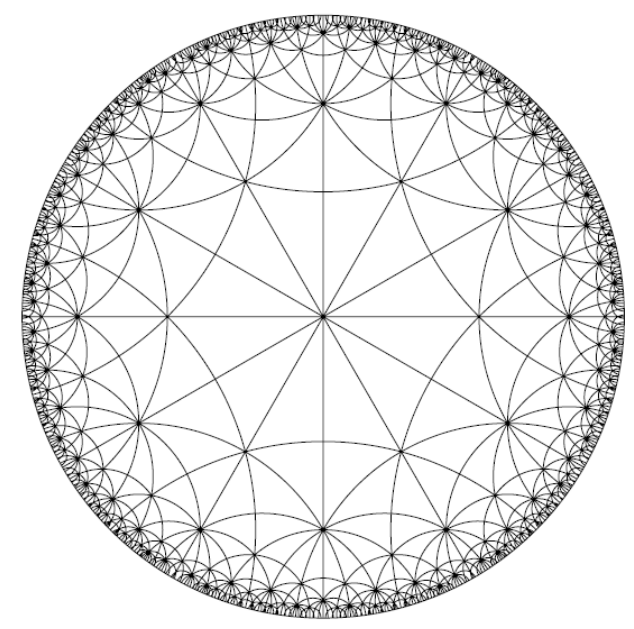

Figure 3. A tessellation of $B^{2}$ by the fundamental polyhedron $T(2,4,6)$ of $W(2,4,6)$ (image taken from Rat06]).

The fact that a hyperbolic reflection group can be completely reconstructed from the faces of its fundamental polyhedron can be used to obtain a handy description of the group in terms of the normal vectors (with respect to the faces). In formulating the next definitions and results, we allow any system of vectors $\left(v_{i}\right)_{i \in I}$ that satisfies the conditions $\left(v_{i}, v_{i}\right)>0$ and $\left(v_{i}, v_{j}\right) \leq 0$ for $i, j \in I$ (which is automatically fulfilled by the normal vectors). This allows the following concepts to be applied later in some proofs of non-reflectivity.

DEFINITION 3.5. Let $\left(v_{i}\right)_{i \in I}$ be a system of vectors in $V$ with the property $\left(v_{i}, v_{i}\right)>0$ and $\left(v_{i}, v_{j}\right) \leq 0$ for $i, j \in I$. The matrix

$$
G:=\left(g_{i j}\right)_{i, j \in I}, \quad \text { with } \quad g_{i j}:=\frac{\left(v_{i}, v_{j}\right)}{\sqrt{\left(v_{i}, v_{i}\right)\left(v_{j}, v_{j}\right)}}
$$

is called the (normalized) Gram matrix of the system $\left(v_{i}\right)_{i \in I}$. For a subset $J \subseteq I$, we write $G_{J}:=\left(g_{i j}\right)_{i, j \in J}$.

All relevant geometric properties of the hyperplanes $h_{v_{i}}$ are stored in the normalized Gram matrix and can be extracted form $G$ in the following way.

Proposition 3.6. Let $\left(v_{i}\right)_{i \in I}$ be a system of vectors in $V$ with the property $\left(v_{i}, v_{i}\right)>0$ and $\left(v_{i}, v_{j}\right) \leq 0$ for $i, j \in I$ and let $G=\left(g_{i j}\right)_{i, j \in I}$ be the associated Gram matrix. Then

a) $g_{i j}<-1 \Longrightarrow h_{v_{i}}$ and $h_{v_{j}}$ diverge,

b) $g_{i j}=-1 \Longrightarrow h_{v_{i}}$ and $h_{v_{j}}$ are parallel, 
c) $g_{i j}>-1 \Longrightarrow h_{v_{i}}$ and $h_{v_{j}}$ intersect. Furthermore, if we write $\varangle\left(v_{i}, v_{j}\right)$ for the dihedral angle between $h_{v_{i}}$ and $h_{v_{j}}$, then

$$
\cos \varangle\left(v_{i}, v_{j}\right)=-g_{i j} .
$$

Proof. See [Rat06], §3.2.

Apart from the normalized Gram matrix, there is another common way to describe reflection groups and their fundamental domain. The so-called Coxeter diagram contains less information than $G$ but enough to determine the combinatorial structure of the fundamental polyhedron and the presentation of the associated hyperbolic reflection group in terms of generators and relations.

Definition 3.7. The Coxeter diagram of the vector system $\left(v_{i}\right)_{i \in I}$ is a graph $\Gamma$ with vertices $v_{i}, i \in I$, and edges defined as follows:

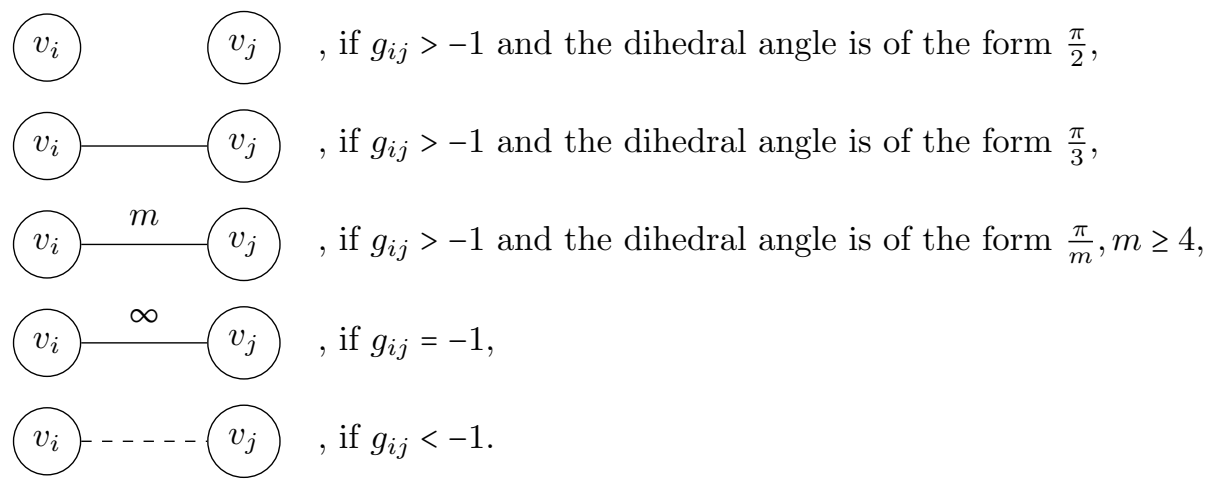

For a subset $J \subseteq I$, we write $\Gamma_{J}$ for the subgraph containing the vertices $v_{j}, j \in J$, and the corresponding edges.

EXAmPLE 3.8. If we look back at example 3.4 , we see that the Coxeter diagram of a group $W(a, b, c)$ is either
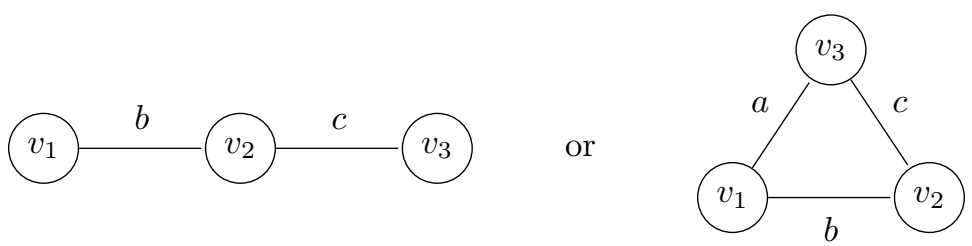

according as $a=2$ or $a>2$, where $v_{1}, v_{2}, v_{3}$ correspond to the normal vectors perpendicular to the edges of $T(a, b, c)$.

We can now formulate a very useful criterion, which characterizes the combinatorial structure of a fundamental polyhedron by elementary properties of the normalized Gram matrix and the Coxeter diagram. 
THEOREM 3.9. Let $\left(v_{i}\right)_{i \in I}$ be a system of vectors in $V$ with the property $\left(v_{i}, v_{i}\right)>$ 0 and $\left(v_{i}, v_{j}\right) \leq 0$ for $i, j \in I$. Let $P:=\bigcap_{i \in I} h_{v_{i}}^{+}$be the corresponding polyhedron, $G$ be the associated Gram matrix and $\Gamma$ the corresponding Coxeter diagram. For a subset $J \subseteq I$ we have:

a) $P \cap\left(\bigcap_{j \in J} h_{v_{j}}^{+}\right)$is a face of codimension $|J|$ iff $G_{J}$ is positive-definite.

b) Let $\Gamma_{J}=\bigcup_{k} \Gamma_{J_{k}}, J_{k} \subseteq J$, be the decomposition of $\Gamma$ into connected components. Then $P \cap\left(\cap_{j \in J} h_{v_{j}}^{+}\right)$is a cusp (vertex at infinity) iff $G_{J}$ is of rank $n-1$ and $G_{J_{k}}$ is positive-semidefinite with one-dimensional radical for every connected component $k$.

Proof. See [Vin72a].

\subsection{Arithmetic Reflection Groups and Reflective Lorentzian Lattices}

Arithmetic groups are arguably the most interesting subclass of hyperbolic reflection groups. Their theoretical background is not restricted (but closely related) to hyperbolic reflections and much deeper than the one presented here. We only mention that arithmetic groups in general are subgroups of semisimple Lie groups defined using algebraic groups. The classification of semisimple algebraic groups (cf. Tit66]) divides the possible types of arithmetic groups into three classes. As Vinberg showed in [Vin67], if an arithmetic group is generated by hyperbolic reflections, then it is automatically from the simplest type, meaning that it is commensurable to the isometry group of an indefinite lattice of signature $(n, 1)$ over some algebraic number field. An arithmetic group (of the simplest type) has a noncompact fundamental domain if and only if it is defined over $\mathbb{Q}$ and the indefinite lattice is isotropic (this was shown in BH62]). Since we are only interested in

non-cocompact groups on the hyperbolic 5-space, the theorem of Hasse-Minkowski implies that we only need to consider the number field $\mathbb{Q}$.

DEFinition 3.10. An integral $\mathbb{Z}$-lattice of signature $(n, 1)$ is called a Lorentzian lattice.

DEFinition 3.11. A hyperbolic reflection group $W$ is called arithmetic if there exists a Lorentzian lattice $E$ such that $W$ is of finite index in $O^{+}(E)$.

In order to investigate the connections between arithmetic reflection groups and Lorentzian lattices, we need to adapt the notion of root systems to the indefinite case (keep in mind that in chapter 1 root systems were only defined for positivedefinite lattices).

Definition 3.12. Let $(E, b)$ be a Lorentzian lattice.

a) A primitive vector $v \in E$ is called root of $E$ if $s_{v}(E)=E$ and $(v, v)>0$. 
b) The set $R(E):=\{v \in E \mid v$ is a root of $E\}$ is called root system of $E$.

c) The subgroup $W(E) \leqslant O(E)$ generated by all reflections $s_{v}$, with $v \in$ $R(E)$, is called Weyl group of $E$.

Abbreviatory, we write $O^{+}(E):=O(E) \cap O^{+}(V)$.

Proposition 3.13. Let $E$ be a Lorentzian lattice. The Weyl group $W(E)$ is a hyperbolic reflection group.

Proof. Only the discreteness of $W(E)$ in $O^{+}(V)$ remains to be shown. This follows from the fact that $E$ is a lattice on $V$.

Obviously, the condition $(v, v)>0$ is redundant in the positive-definite case but here it is made for the following reason: $(v, v)>0$ iff $s_{v} \in O^{+}(V)$. In contrast to the positive-definite case, $R(E)$ is not a root system in the sense of Lie algebra; in general $R(E)$ is not a finite set. Nevertheless, the major concept of fundamental roots can be transfered to the Lorentzian case. A set of fundamental roots for $R(E)$ is by definition obtained as follows. The set of all reflecting hyperplanes $h_{v}:=\left\{x \in H^{n} \mid(x, v)=0\right\}, v \in R(E)$, is locally finite and divides $H^{n}$ into connected components. Actually, the group $W(E)$ acts transitively on the connected components of $H^{n} \backslash \cup h_{v}$ (the so-called open chambers). Pick an open chamber $P(E)^{\circ}$ and let $P(E)$ be its closure. The set $P(E)$ is of the form

$$
P(E)=\bigcap_{v \in \tilde{R}(E)} h_{v}^{+}
$$

for a unique minimal subset $\tilde{R}(E) \subset R(E)$ (consider the roots belonging to the supporting hyperplanes of $P(E)$, cf. Rat06, Theorem 6.3.2).

Definition 3.14. Let $E$ be a Lorentzian lattice. The elements of $\tilde{R}(E)$ constructed as above are called fundamental roots of $E$ (depending on the choice of $P(E))$.

Recall that a hyperbolic reflection group $W$ is called maximal if there is no other hyperbolic reflecting group $W^{\prime}$ such that $W \varsubsetneqq W^{\prime} \subseteq O^{+}(V)$. As it will turn out, Lorentzian lattices with a finite set of fundamental roots are precisely those which can be used to describe maximal arithmetic reflection groups. For now, we make the following

Definition 3.15. A Lorentzian lattice $E$ is called reflective if $W(E)$ is of finite index in $O^{+}(E)$.

Proposition 3.16.

a) If $E$ is a reflective Lorentzian lattice, then the Weyl group $W(E)$ is an arithmetic hyperbolic reflection group. 
b) If $W$ is a maximal arithmetic reflection group, then there is a Lorentzian lattice $E$ with $W=W^{+}(E)$.

Proof. Both a) and b) follow directly from the definition.

Similar to positive-definite lattices, the reflectivity of a Lorentzian lattice is characterized by a finiteness condition regarding its root system.

Lemma 3.17. Let $E$ be a Lorentzian lattice with root system $R(E)$ and fundamental polyhedron $P(E)$. Let $\operatorname{Sym}(P(E)):=\left\{\varphi \in O^{+}(V) \mid \varphi(P(E))=P(E)\right\}$ be the symmetry group of $P(E)$. Then

$$
O^{+}(E)=W(E) \rtimes H,
$$

with $H:=\operatorname{Sym}(P(E)) \cap O^{+}(E)$.

Proof. See [Vin72b], Proposition 3.

TheOREm 3.18. Let $E$ be a Lorentzian lattice with root system $R(E)$. Then $E$ is reflective iff the set of fundamental roots $\tilde{R}(E)$ is finite.

Proof. This follows from lemma 3.17.

Given a Lorentzian lattice $E$, we wish to calculate all fundamental roots in order to decide whether the set $\tilde{R}(E)$ is finite, or equivalently, whether the arithmetic group $O^{+}(E)$ is generated up to finite index by reflections. The main tool for this is Vinberg's algorithm which we have implemented in MAGMA (cf. [BCP97]) and we review now (cf. Vin72a $)$.

The algorithm starts by picking an arbitrary point $p_{0} \in H^{n}$, the so-called controlling vector. Consider all roots of $E$ the reflecting hyperplanes of which pass through $p_{0}$,

$$
R^{\prime}:=\left\{v \in R(E) \mid\left(v, p_{0}\right)=0\right\} .
$$

The set $R^{\prime}$ is contained in the root system of the positive-definite lattice $p_{0}^{\perp} \cap E$. In fact, $R^{\prime}$ is itself a crystallographic root system (in the usual sense of Lie algebra). Thus we can pick a root basis $v_{1}, \ldots, v_{n^{\prime}}\left(n^{\prime} \leq n\right)$ of $R^{\prime}$. The group generated by the hyperbolic reflections $\sigma_{v_{1}}, \ldots, \sigma_{v_{n}^{\prime}}$ is the stabilizer of $p_{0}$ in $W(E)$ and its fundamental polyhedron is of the form

$$
P_{0}:=\bigcap_{i=1}^{n^{\prime}} h_{v_{i}}^{+} .
$$

There is a fundamental polyhedron $P(E)$ of $W(E)$ which is located in $P_{0}$ and contains $p_{0}$ (one can choose $p_{0}$ to be a vertex of this polyhedron). The hyperplanes $h_{v_{i}}(i=1, \ldots, n)$ bounding $P_{0}$ are bounding $P(E)$ as well, but additional hyperplanes are needed. These required hyperplanes are exactly those perpendicular to 
the fundamental roots of $E$ and are found in the following way. If $v_{1}, \ldots, v_{m}$, with $m \geq n$, are already chosen, a root $v \in R(E)$ can then be picked such that $\left(v, p_{0}\right)<0$, $\left(v, v_{i}\right) \leq 0$ for all $i=1, \ldots, m$, and the distance between $p_{0}$ and $h_{v}$ is the smallest possible, i.e. the quantity

$$
\sinh ^{2}\left(\operatorname{dist}\left(p_{0}, h_{v}\right)\right)=-\frac{\left(v, p_{0}\right)^{2}}{(v, v)\left(p_{0}, p_{0}\right)}
$$

is minimized (cf. Rat06, Theorem 3.2.12 for some facts about the hyperbolic distance between hyperplanes and points). This way, Vinberg's algorithm will produce a (possibly infinite) sequence $\left(v_{1}, v_{2}, v_{3}, \ldots\right)$ of fundamental roots, which is well-definied up to permutation of the $v_{i}$ with equal $\operatorname{dist}\left(p_{0}, h_{v}\right)$. Obviously, if the lattice is not reflective, then the algorithm will not terminate. In the next section, we will see how to recognize this situation. We will also give criteria which decide whether a set of finitely many roots produced by the algorithm already make up the whole set $\tilde{R}(E)$.

\subsection{Methods to Prove Reflectivity and Non-Reflectivity}

Given a Lorentzian lattice $E$, a controlling vector $p_{0} \in H^{n}$ and a root basis for the root system $\left\{v \in R(E) \mid\left(v, p_{0}\right)=0\right\}$, there is a unique extension of these roots to a set of fundamental roots for $R(E)$. As mentioned in the previous section, Vinberg's algorithm will find this extension by iteratively adjoining new fundamental roots. If $E$ is reflective, then the set of fundamental roots is finite and after some number of iterations the algorithm will have found them all. The next two lemmata show that one can diagnose this situation by inspecting the volume of the polyhedron defined by the so-far-found fundamental roots.

Lemma 3.19. Let $E$ be a Lorentzian lattice with fundamental polyhedron $P(E)$. Then $E$ is reflective iff $P(E)$ has finite volume.

Proof. This follows from Vin72a, Proposition 3 and the fact that the arithmetic group $O^{+}(E)$ always has a fundamental polyhedron of finite volume. This is a well-known general fact, which follows from reduction theory.

Lemma 3.20. Let $E$ be a Lorentzian lattice and $\left\{v_{1}, \ldots, v_{m}\right\} \subseteq \tilde{R}(E)$ a finite set of fundamental roots of $R(E)$ generated by Vinberg's algorithm. If $P_{m}:=\cap_{i=1}^{m} h_{v_{i}}^{+}$ has finite volume then $P(E)=P_{m}$.

Proof. See [Vin72a], Proposition 5.

As the next proposition shows, the finiteness of the volume can be characterized by certain combinatorial properties. 
Proposition 3.21. Let $P$ be a Coxeter polyhedron. Then $P$ has finite volume iff on every edge of $P$ there lie two (different) vertices or cusps.

Proof. See [Vin85, Proposition 4.2.

Recall theorem 3.9 where the combinatorial structure of the fundamental polyhedron is characterized by elemental properties of the normalized Gram matrix and the Coxeter diagram. In combination with above proposition, this gives us a very effective tool to check a Lorentzian lattice for reflectivity. We have implemented this procedure in MAGMA (cf. [BCP97]).

If our MAGMA implementation of Vinberg's algorithm produces, say, more than $10^{3}$ fundamental roots then we can expect the lattice to be non-reflective. We will now present two methods to prove this rigorously. The first one consists of realizing non-reflective lattices of smaller dimension as orthogonal summands.

Theorem 3.22. Let $E$ be a reflective Lorentzian lattice. Then every Lorentzian orthogonal summand of $E$ is reflective.

Proof. See Bug92, §2.

The major assistance to this method is the classification of reflective Lorentzian lattices in the hyperbolic dimension 3 by Scharlau (cf. [Sch89) and the hyperbolic dimension 4 by Walhorn (cf. Wal93). The question whether a non-reflective lattice of smaller dimension is embeddable into a larger one can be answered in a simple manner by our structure theorem 4.3 .

The second method consists in finding symmetries of infinite order of the polyhedron defined by some so-far-found fundamental roots. This is done by inspecting the automorphism group of the Coxeter diagram.

Lemma 3.23. Let $H \leqslant O^{+}(V)$ be a discrete subgroup. Let

$$
F i x(H):=\{v \in V \mid \forall \varphi \in H: \varphi(v)=v\}
$$

be a set of fixed vectors of $H$ and $C:=\{v \in V \mid(v, v)<0\}$. The group $H$ is infinite iff $\operatorname{Fix}(H) \cap C=\varnothing$.

Proof. See Bug92, Lemma 3.1.

According to lemma 3.17 we can apply the above lemma to $H:=\operatorname{Sym}(P(E)) \cap$ $\mathrm{O}^{+}(E)$. Elements of $H$ can be found with the following theorem.

Theorem 3.24. Let $E$ be a Lorentzian lattice and $\left\{v_{1}, \ldots, v_{m}\right\} \subseteq \tilde{R}(E)$ a finite set of fundamental roots of $R(E)$ generated by Vinberg's algorithm. Let $\Gamma_{m}$ be the corresponding subgraph of the Coxeter diagram. If the following conditions hold

1) the vectors $v_{1}, \ldots, v_{m}$ generate $E$, 
2) there is a subset $J \subseteq\{1, \ldots, m\}$ such that $\bigcap_{j \in J} h_{v_{j}}^{+}$is a vertex or a cusp, then every non-trivial graph automorphism of $\Gamma_{m}$ induces an non-trivial element of $\operatorname{Sym}(P(E)) \cap O^{+}(E)$.

Proof. See Bug92, Lemma 3.3.

We have automated and implemented the calculation of the automorphism group of a Coxeter diagram and the search for suitable automorphisms in MAGMA. 



\section{CHAPTER 4}

\section{Classification of Reflective Lorentzian Lattices}

\subsection{Connections between Reflective Lorentzian Lattices and Totally-Reflective Genera}

In this section we want to describe how to use the classification of totallyreflective genera from the first part of this work to obtain insights on the structure of reflective Lorentzian lattices and arithmetic reflection groups. We show that a reflective Lorentzian lattice possesses a decomposition into an indefinite and a positive-definite part. The indefinite part is simply a scaled hyperbolic plane (in the sense of the theory of quadratic forms, not to be confused with the hyperbolic plane from the previous chapter) and the positive part turns out to be totally-reflective. We start with Vinberg's lemma, which relates the two notions of reflectivity of lattices to each other.

Lemma 4.1 (cf. Vin72b]). Let $E$ be a reflective Lorentzian lattice. Then, for every isotropic vector $v \in E$, the positive-definite lattice $v^{\perp} / \mathbb{Z} v$ is reflective.

In the following, we will write $\mathbb{H}$ for the hyperbolic plane, that is the integral $\mathbb{Z}$-lattice with Gram matrix $\left(\begin{array}{ll}0 & 1 \\ 1 & 0\end{array}\right)$.

COROLlaRY 4.2. Let $E$ be a reflective Lorentzian lattice of the form

$$
E={ }^{\alpha} \mathbb{H} \perp L,
$$

with $L$ being a positive-definite $\mathbb{Z}$-lattice and $\alpha \in \mathbb{Q}$. Then $L$ is totally-reflective.

Proof. Notice that $E={ }^{a} \mathbb{H} \perp L$ only depends on the genus of $L$. By this we mean that

$$
{ }^{\alpha} \mathbb{H} \perp L \cong{ }^{\alpha} \mathbb{H} \perp L^{\prime},
$$

for every $L^{\prime} \in \mathcal{G}(L)$. This follows from

$$
\left({ }^{\alpha} \mathbb{H} \perp L\right) \otimes \mathbb{Z}_{p} \cong\left({ }^{\alpha} \mathbb{H} \perp L^{\prime}\right) \otimes \mathbb{Z}_{p},
$$

for all $p \in \mathbb{P} \cup\{\infty\}$, and the fact that lattices of the form $\mathbb{H} \perp L$ (with $\operatorname{dim} L \geq 1$ ) are always of class number one (cf. [Kne56]). The assertion follows then from Vinberg's lemma with $v$ chosen to be an isotropic basis vector of $\mathbb{H}$. 


\section{TheOREM 4.3.}

a) Let $E$ be a strongly square-free reflective Lorentzian lattice of signature $(5,1)$. Then $E$ is of the form $E={ }^{\alpha} \mathbb{H} \perp L$ with $L$ a square-free totallyreflective lattice of dimension 4 and $\alpha \in\{1,2\}$. The scaling factor $\alpha=2$ only occurs if the 2-adic symbol of $E$ is of the form $\mathrm{I}_{5,1}\left(1^{\varepsilon_{0}, 4} 2_{\mathrm{II}}^{\varepsilon_{1}, 2}\right)$.

b) Let $W$ be a maximal arithmetic reflection group on the hyperbolic 5-space. Then $W$ is of the form $W=W\left({ }^{\alpha} \mathbb{H} \perp L\right)$ with $L$ a square-free totallyreflective lattice of dimension 4 and $\alpha \in\{1,2\}$.

Proof. a) Let $E$ be a strongly square-free Lorentzian lattice of signature $(5,1)$. We want so show that ${ }^{\alpha} \mathbb{H}$ splits off in $E$. Because the class number of $E$ is one, it is sufficient to show that ${ }^{\alpha} \mathbb{H}$ splits off at every prime $p \in \mathbb{P}$. The Jordan decomposition of $E$ is of the form $E \otimes \mathbb{Z}_{p}=E_{0} \perp{ }^{p} E_{1}$, with $\operatorname{dim} E_{0} \in\{3,4,5,6\}$ and $\operatorname{dim} E_{1}=$ $6-\operatorname{dim} E_{0}$. If $p \neq 2$ then ['M00], $92: 1$, implies that $\mathbb{H}$ and ${ }^{2} \mathbb{H}$ split of in $E_{0}$ and thus in $E \otimes \mathbb{Z}_{p}$. Now let $p=2$. Dimensionwise, the following 2-adic symbols are possible:

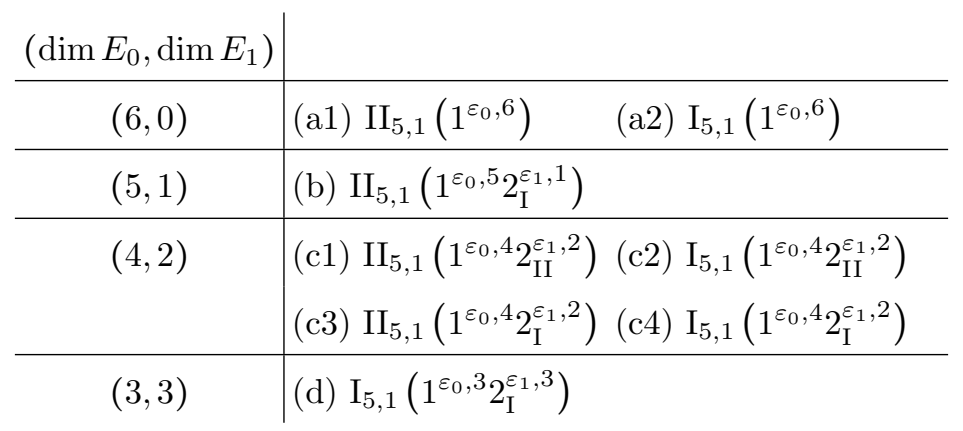

In the following, $A_{2}$ is the binary even lattice with Gram matrix $\left(\begin{array}{ll}2 & 1 \\ 1 & 2\end{array}\right)$.

"(a1), (a2), (b), (c1), (c3)": Kit93, Theorem 5.2.5 and Proposition 5.2.3 imply that $\mathbb{H}$ splits off in $E_{0}$.

"(c2)": We want to show that ${ }^{2} \mathbb{H}$ splits off. The unimodular Jordan component is of the form $E_{0}=\mathbb{H} \perp\langle a, b\rangle$ or $E_{0}=A_{2} \perp\langle a, b\rangle$ with $a, b \in \mathbb{Z}_{2}$ odd. The 2-modular component is of the form $E_{1}=\mathbb{H}$ or $E_{1}=A_{2}$. If $E_{1}=\mathbb{H}$ then ${ }^{2} \mathbb{H}$ splits off in $E \otimes \mathbb{Z}_{2}$. If $E_{1}=A_{2}$ then the following isometries show that, again, ${ }^{2} \mathbb{H}$ splits off in $E \otimes \mathbb{Z}_{2}$ :

$$
E \otimes \mathbb{Z}_{2}= \begin{cases}A_{2} \perp\langle a, b\rangle \perp{ }^{2} A_{2} & \cong A_{2} \perp\langle a+4, b\rangle \perp{ }^{2} \mathbb{H}, \\ \mathbb{H} \perp\langle a, b\rangle \perp{ }^{2} A_{2} & \cong \mathbb{H} \perp\langle a+4, b\rangle \perp{ }^{2} \mathbb{H} .\end{cases}
$$

"(c4)": We want to show that $\mathbb{H}$ splits off. The lattice $E \otimes \mathbb{Z}_{2}$ is of the form $E \otimes \mathbb{Z}_{2}=\mathbb{H} \perp\langle a, b\rangle \perp{ }^{2}\langle x, y\rangle$ or $E \otimes \mathbb{Z}_{2}=A_{2} \perp\langle a, b\rangle \perp{ }^{2}\langle x, y\rangle$ with $a, b, x, y \in \mathbb{Z}_{2}$ odd. In the first case the assertion is clear. In the second case the assertion follows from

$$
A_{2} \perp\langle a, b\rangle \perp{ }^{2}\langle x, y\rangle \cong \mathbb{H} \perp\langle a, b\rangle \perp^{2}\langle x+4, y\rangle .
$$


"(d)": We want to show that $\mathbb{H}$ splits off. The unimodular Jordan component is of the form $E_{0}=\mathbb{H} \perp\langle a\rangle$ or $E_{0}=A_{2} \perp\langle a\rangle$ with $a \in \mathbb{Z}_{2}$ odd. In the first case the assertion is clear. In the second case, let $E_{1}$ be either $\mathbb{H} \perp\langle b\rangle$ or $A_{2} \perp\langle b\rangle$ with $b \in \mathbb{Z}_{2}$ odd. Then the following isometries imply the assertion:

$$
E \otimes \mathbb{Z}_{2}= \begin{cases}A_{2} \perp\langle a\rangle \perp{ }^{2}(\mathbb{H} \perp\langle b\rangle) & \cong \mathbb{H} \perp\langle a+4\rangle \perp{ }^{2}\left(A_{2} \perp\langle b+4\rangle\right), \\ A_{2} \perp\langle a\rangle \perp{ }^{2}\left(A_{2} \perp\langle b\rangle\right) & \cong \mathbb{H} \perp\langle a+4\rangle \perp{ }^{2}(\mathbb{H} \perp\langle b+4\rangle) .\end{cases}
$$

All isometries above are defined over $\mathbb{Z}_{2}$ and, thus, can be checked by calculating the 2-adic symbol. In every case, $E$ can be written as $E={ }^{\alpha} \mathbb{H} \perp L$ with $\alpha \in\{1,2\}$. Corollary 4.2 implies that $L$ is totally-reflective.

b) Since $W$ is maximal and arithmetic one can find a Lorentzian lattice $E$ with $W=W(E)$. After repeated use of $\mathrm{D}_{p}$ and $\mathrm{E}_{p}$ the lattice $E$ can be transformed into a strongly square-free lattice without changing $W$. The assertion then follows from a).

A similar structure theorem appears for the first time in $\mathbf{S c h 8 9}$ and later in Wal93. (for Lorentzian lattices in dimension 4 and 5).

\subsection{Classification Result for Reflective Lorentzian Lattices}

With the methods developed above, the classification of strongly square-free, reflective Lorentzian lattice could be carried out computationally in MAGMA. We have picked a lattice $L$ from every square-free totally-reflective genus and have applied Vinberg's algorithm to $E={ }^{\alpha} \mathbb{H} \perp L$. As shown in lemma 3.19 and lemma 3.20 . if the algorithm terminates, then $E$ is proven to be reflective. If the algorithm does not terminate (we waited for around $10^{3}$ roots to be found), then non-reflectivity is proven with the methods discussed in section 3.3.

TheOREM 4.4. The Lorentzian lattices of signature $(5,1)$ in the table 1 below are reflective. Every strongly square-free, reflective Lorentzian lattice of signature $(5,1)$ is isometric to one in that table.

COROLlary 4.5. Every maximal arithmetic reflection group $W$ on $H^{5}$ is of the form $W=W(E)$ with $E$ being a Lorentzian lattice from the table below.

Proof. This follows from the structure theorem 4.3 and above classification.

The notation ${ }^{\alpha} \mathbb{H} \perp$ Genus means that $L$ can be chosen arbitraryly within the given 4-dimensional genus. The combinatorial structure of the fundamental polyhedron is given as follows:

$r=$ Number of fundamental roots $=$ Number of 4-dimensional faces, $f_{3}=$ Number of 3-dimensional faces, 


$$
\begin{aligned}
f_{2} & =\text { Number of 2-dimensional faces, } \\
e & =\text { Number of edges, } \\
v & =\text { Number of vertices, } \\
c & =\text { Number of cusps (vertices at infinity). }
\end{aligned}
$$

The lattices are ordered by the parities of the 2-adic Jordan components of $L$.

\begin{tabular}{c|c|c|c|c|c|c|c|c} 
No. & - det & Lattice & $r$ & $f_{3}$ & $f_{2}$ & $e$ & $v$ & $c$ \\
\hline & & & & & & & & \\
1 & 5 & $\mathbb{H} \perp \mathrm{II}\left(5^{+1}\right)$ & 6 & 15 & 20 & 15 & 5 & 1 \\
2 & 9 & $\mathbb{H} \perp \mathrm{II}\left(3^{+2}\right)$ & 7 & 21 & 33 & 27 & 9 & 1 \\
3 & 21 & $\mathbb{H} \perp \mathrm{II}\left(3^{-1} 7^{+1}\right)$ & 9 & 32 & 57 & 51 & 18 & 1 \\
4 & 25 & $\mathbb{H} \perp \mathrm{II}\left(5^{-2}\right)$ & 9 & 33 & 61 & 57 & 21 & 1 \\
5 & 45 & $\mathbb{H} \perp \mathrm{II}\left(3^{-2} 5^{+1}\right)$ & 12 & 50 & 98 & 92 & 30 & 4 \\
6 & 49 & $\mathbb{H} \perp \mathrm{II}\left(7^{+2}\right)$ & 16 & 80 & 176 & 176 & 64 & 2 \\
7 & 125 & $\mathbb{H} \perp \mathrm{II}\left(5^{+3}\right)$ & 10 & 40 & 80 & 80 & 30 & 2 \\
8 & 1 & $\mathbb{H} \perp \mathrm{I}\left(1^{+4}\right)$ & 6 & 15 & 20 & 15 & 5 & 1 \\
9 & 3 & $\mathbb{H} \perp \mathrm{I}\left(3^{-1}\right)$ & 7 & 21 & 33 & 27 & 9 & 1 \\
10 & 3 & $\mathbb{H} \perp \mathrm{I}\left(3^{+1}\right)$ & 8 & 25 & 40 & 34 & 12 & 1 \\
11 & 5 & $\mathbb{H} \perp \mathrm{I}\left(5^{+1}\right)$ & 8 & 25 & 40 & 34 & 11 & 2 \\
12 & 5 & $\mathbb{H} \perp \mathrm{I}\left(5^{-1}\right)$ & 9 & 32 & 57 & 51 & 18 & 1 \\
13 & 7 & $\mathbb{H} \perp \mathrm{I}\left(7^{+1}\right)$ & 11 & 42 & 77 & 70 & 24 & 2 \\
14 & 9 & $\mathbb{H} \perp \mathrm{I}\left(3^{+2}\right)$ & 8 & 28 & 50 & 44 & 14 & 2 \\
15 & 9 & $\mathbb{H} \perp \mathrm{I}\left(3^{-2}\right)$ & 9 & 32 & 57 & 51 & 16 & 3 \\
16 & 15 & $\mathbb{H} \perp \mathrm{I}\left(3^{+1} 5^{+1}\right)$ & 16 & 74 & 153 & 148 & 52 & 3 \\
17 & 15 & $\mathbb{H} \perp \mathrm{I}\left(3^{-1} 5^{-1}\right)$ & 15 & 66 & 131 & 122 & 40 & 4 \\
18 & 15 & $\mathbb{H} \perp \mathrm{I}\left(3^{+1} 5^{-1}\right)$ & 12 & 54 & 114 & 113 & 42 & 1 \\
19 & 25 & $\mathbb{H} \perp \mathrm{I}\left(5^{-2}\right)$ & 21 & 120 & 282 & 288 & 102 & 5 \\
20 & 25 & $\mathbb{H} \perp \mathrm{I}\left(5^{+2}\right)$ & 14 & 67 & 144 & 142 & 46 & 7 \\
21 & 27 & $\mathbb{H} \perp \mathrm{I}\left(3^{+3}\right)$ & 9 & 34 & 64 & 58 & 18 & 3 \\
22 & 27 & $\mathbb{H} \perp \mathrm{I}\left(3^{-3}\right)$ & 9 & 34 & 64 & 58 & 18 & 3 \\
23 & 75 & $\mathbb{H} \perp \mathrm{I}\left(3^{+1} 5^{-2}\right)$ & 86 & 672 & 1788 & 1902 & 660 & 42 \\
24 & 125 & $\mathbb{H} \perp \mathrm{I}\left(5^{+3}\right)$ & 20 & 115 & 280 & 295 & 100 & 12 \\
25 & 2 & $\mathbb{H} \perp \mathrm{I}\left(2_{1}^{+1}\right)$ & 7 & 20 & 30 & 24 & 8 & 1 \\
26 & 6 & $\mathbb{H} \perp \mathrm{I}\left(2_{1}^{+1} 3^{+1}\right)$ & 9 & 32 & 57 & 51 & 18 & 1 \\
27 & 6 & $\mathbb{H} \perp \mathrm{I}\left(2_{1}^{+1} 3^{-1}\right)$ & 9 & 31 & 53 & 45 & 14 & 2 \\
28 & 10 & $\mathbb{H} \perp \mathrm{I}\left(2_{1}^{+1} 5^{-1}\right)$ & 12 & 49 & 94 & 86 & 28 & 3
\end{tabular}


4.2. CLASSIFICATION RESULT FOR REFLECTIVE LORENTZIAN LATTICES 49

\begin{tabular}{|c|c|c|c|c|c|c|c|}
\hline 29 & 14 & $\mathbb{H} \perp \mathrm{I}\left(2_{1}^{+1} 7^{+1}\right)$ & 15 & 67 & 135 & 127 & 42 \\
\hline 30 & 18 & $\mathbb{H} \perp \mathrm{I}\left(2_{1}^{+1} 3^{-2}\right)$ & 14 & 62 & 125 & 116 & 36 \\
\hline 31 & 18 & $\mathbb{H} \perp \mathrm{I}\left(2_{1}^{+1} 3^{+2}\right)$ & 12 & 51 & 101 & 93 & 30 \\
\hline 32 & 54 & $\mathbb{H} \perp \mathrm{I}\left(2_{1}^{+1} 3^{+3}\right)$ & 16 & 75 & 156 & 145 & 42 \\
\hline 33 & 54 & $\mathbb{H} \perp \mathrm{I}\left(2_{1}^{+1} 3^{-3}\right)$ & 18 & 99 & 230 & 231 & 78 \\
\hline 34 & 4 & $\mathbb{H} \perp \mathrm{II}\left(2_{\mathrm{II}}^{-2}\right)$ & 6 & 15 & 20 & 15 & 5 \\
\hline 35 & 20 & $\mathbb{H} \perp \mathrm{II}\left(2_{\mathrm{II}}^{+2} 5^{+1}\right)$ & 8 & 25 & 40 & 34 & 11 \\
\hline 36 & 20 & $\mathbb{H} \perp \mathrm{II}\left(2_{\mathrm{II}}^{-2} 5^{-1}\right)$ & 8 & 25 & 40 & 34 & 12 \\
\hline 37 & 36 & $\mathbb{H} \perp \operatorname{II}\left(2_{\mathrm{II}}^{-2} 3^{-2}\right)$ & 7 & 21 & 33 & 27 & 8 \\
\hline 38 & 36 & $\mathbb{H} \perp \mathrm{II}\left(2_{\mathrm{II}}^{+2} 3^{+2}\right)$ & 8 & 28 & 50 & 44 & 14 \\
\hline 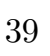 & 84 & $\mathbb{H} \perp \mathrm{II}\left(2_{\mathrm{II}}^{-2} 3^{+1} 7^{+1}\right)$ & 16 & 74 & 153 & 148 & 52 \\
\hline 40 & 100 & $\mathbb{H} \perp \operatorname{II}\left(2_{\mathrm{II}}^{-2} 5^{+2}\right)$ & 9 & 33 & 61 & 57 & 19 \\
\hline 41 & 100 & $\mathbb{H} \perp \mathrm{II}\left(2_{\mathrm{II}}^{+2} 5^{-2}\right)$ & 21 & 120 & 282 & 288 & 102 \\
\hline 42 & 180 & $\mathbb{H} \perp \mathrm{II}\left(2_{\mathrm{II}}^{-2} 3^{+2} 5^{+1}\right)$ & 16 & 80 & 177 & 178 & 64 \\
\hline 43 & 196 & $\mathbb{H} \perp \mathbb{I I}\left(2_{\mathrm{II}}^{-2} 7^{-2}\right)$ & 15 & 72 & 156 & 159 & 54 \\
\hline 44 & 500 & $\mathbb{H} \perp \mathrm{II}\left(2_{\mathrm{II}}^{+2} 5^{+3}\right)$ & 20 & 115 & 280 & 295 & 100 \\
\hline 45 & 500 & $\mathbb{H} \perp \mathrm{II}\left(2_{\mathrm{II}}^{-2} 5^{-3}\right)$ & 12 & 56 & 124 & 126 & 44 \\
\hline 46 & 12 & $\mathbb{H} \perp \mathrm{II}\left(2_{2}^{+2} 3^{-1}\right)$ & 7 & 21 & 33 & 27 & 9 \\
\hline 47 & 12 & $\mathbb{H} \perp \mathrm{II}\left(2_{6}^{+2} 3^{+1}\right)$ & 8 & 25 & 40 & 34 & 12 \\
\hline 48 & 28 & $\mathbb{H} \perp \mathrm{II}\left(2_{2}^{+2} 7^{+1}\right)$ & 11 & 42 & 77 & 70 & 24 \\
\hline 49 & 36 & $\mathbb{H} \perp \operatorname{II}\left(2_{0}^{+2} 3^{+2}\right)$ & 10 & 38 & 69 & 59 & 17 \\
\hline 50 & 60 & $\mathbb{H} \perp \mathrm{II}\left(2_{2}^{+2} 3^{+1} 5^{+1}\right)$ & 16 & 74 & 153 & 148 & 52 \\
\hline 51 & 60 & $\mathbb{H} \perp \mathrm{II}\left(2_{2}^{+2} 3^{-1} 5^{-1}\right)$ & 15 & 66 & 131 & 122 & 40 \\
\hline 52 & 60 & $\mathbb{H} \perp \mathrm{II}\left(2_{6}^{+2} 3^{+1} 5^{-1}\right)$ & 12 & 54 & 114 & 113 & 42 \\
\hline 53 & 108 & $\mathbb{H} \perp \mathrm{II}\left(2_{6}^{+2} 3^{-3}\right)$ & 9 & 34 & 64 & 58 & 18 \\
\hline 54 & 108 & $\mathbb{H} \perp \operatorname{II}\left(2_{2}^{+2} 3^{+3}\right)$ & 9 & 34 & 64 & 58 & 18 \\
\hline 55 & 300 & $\mathbb{H} \perp \mathrm{II}\left(2_{2}^{+2} 3^{+1} 5^{-2}\right)$ & 86 & 672 & 1788 & 1902 & 660 \\
\hline 56 & $2^{2} \cdot 1$ & ${ }^{2} \mathbb{H} \perp \mathrm{I}\left(1_{4}^{+4}\right)$ & 6 & 15 & 20 & 15 & 4 \\
\hline 57 & $2^{2} \cdot 3$ & ${ }^{2} \mathbb{H} \perp \mathrm{I}\left(3^{-1}\right)$ & 8 & 26 & 43 & 36 & 11 \\
\hline 58 & $2^{2} \cdot 3$ & ${ }^{2} \mathbb{H} \perp \mathrm{I}\left(3^{+1}\right)$ & 8 & 26 & 43 & 36 & 11 \\
\hline 59 & $2^{2} \cdot 5$ & ${ }^{2} \mathbb{H} \perp \mathrm{I}\left(5^{+1}\right)$ & 11 & 40 & 70 & 62 & 20 \\
\hline ( & $2^{2} \cdot 7$ & ${ }^{2} \mathbb{H} \perp \mathrm{I}\left(7^{+1}\right)$ & 16 & 70 & 138 & 128 & 40 \\
\hline${ }_{1}$ & $2^{2} \cdot 9$ & ${ }^{2} \mathbb{H} \perp \mathrm{I}\left(3^{+2}\right)$ & 11 & 47 & 94 & 87 & 27 \\
\hline 62 & $2^{2} \cdot 9$ & ${ }^{2} \mathbb{H} \perp \mathrm{I}\left(3^{-2}\right)$ & 9 & 33 & 60 & 53 & 15 \\
\hline 63 & $2^{2} \cdot 25$ & ${ }^{2} \mathbb{H} \perp \mathrm{I}\left(5^{+2}\right)$ & 29 & 176 & 420 & 432 & 132 \\
\hline
\end{tabular}




\begin{tabular}{l|c|c|c|c|c|c|c|c}
64 & $2^{2} \cdot 27$ & $2 \mathbb{H} \perp \mathrm{I}\left(3^{+3}\right)$ & 10 & 41 & 82 & 77 & 24 & 4 \\
65 & $2^{2} \cdot 27$ & $2 \mathbb{H} \perp \mathrm{I}\left(3^{-3}\right)$ & 10 & 41 & 82 & 77 & 24 & 4 \\
66 & 4 & $\mathbb{H} \perp \mathrm{I}\left(2_{2}^{+2}\right)$ & 7 & 21 & 33 & 27 & 8 & 2 \\
67 & 12 & $\mathbb{H} \perp \mathrm{I}\left(2_{2}^{+2} 3^{-1}\right)$ & 9 & 33 & 59 & 50 & 14 & 3 \\
68 & 12 & $\mathbb{H} \perp \mathrm{I}\left(2_{2}^{+2} 3^{+1}\right)$ & 11 & 42 & 77 & 68 & 20 & 4 \\
69 & 20 & $\mathbb{H} \perp \mathrm{I}\left(2_{2}^{+2} 5^{-1}\right)$ & 16 & 74 & 151 & 140 & 44 & 5 \\
70 & 36 & $\mathbb{H} \perp \mathrm{I}\left(2_{2}^{+2} 3^{-2}\right)$ & 16 & 83 & 184 & 177 & 54 & 8 \\
71 & 36 & $\mathbb{H} \perp \mathrm{I}\left(2_{2}^{+2} 3^{+2}\right)$ & 11 & 47 & 94 & 87 & 27 & 4 \\
72 & 108 & $\mathbb{H} \perp \mathrm{I}\left(2_{2}^{+2} 3^{+3}\right)$ & 16 & 85 & 193 & 188 & 56 & 10 \\
73 & 108 & $\mathbb{H} \perp \mathrm{I}\left(2_{2}^{+2} 3^{-3}\right)$ & 16 & 85 & 193 & 188 & 56 & 10 \\
74 & 8 & $\mathbb{H} \perp \mathrm{I}\left(2_{3}^{+3}\right)$ & 8 & 25 & 40 & 33 & 10 & 2 \\
75 & 24 & $\mathbb{H} \perp \mathrm{I}\left(2_{3}^{+3} 3^{+1}\right)$ & 11 & 43 & 80 & 71 & 22 & 3 \\
76 & 24 & $\mathbb{H} \perp \mathrm{I}\left(2_{3}^{+3} 3^{-1}\right)$ & 11 & 43 & 80 & 71 & 22 & 3 \\
77 & 72 & $\mathbb{H} \perp \mathrm{I}\left(2_{3}^{+3} 3^{-2}\right)$ & 24 & 128 & 284 & 274 & 84 & 12 \\
78 & 72 & $\mathbb{H} \perp \mathrm{I}\left(2_{3}^{+3} 3^{+2}\right)$ & 14 & 66 & 140 & 134 & 44 & 4 \\
79 & 216 & $\mathbb{H} \perp \mathrm{I}\left(2_{3}^{+3} 3^{+3}\right)$ & 24 & 138 & 324 & 324 & 104 & 12 \\
80 & 216 & $\mathbb{H} \perp \mathrm{I}\left(2_{3}^{+3} 3^{-3}\right)$ & 24 & 138 & 324 & 324 & 104 & 12
\end{tabular}

TABLE 1. Strongly square-free reflective lattices of signature $(5,1)$

\subsection{Examples}

Consider the 4 -dimensional strongly square-free genus I $\left(3^{-1} 5^{-1}\right)$. It contains three lattices, namely

$$
L_{1} \cong\left(\begin{array}{cccc}
1 & 0 & 0 & 0 \\
0 & 1 & 0 & 0 \\
0 & 0 & 1 & 0 \\
0 & 0 & 0 & 15
\end{array}\right), L_{2} \cong\left(\begin{array}{llll}
1 & 0 & 0 & 0 \\
0 & 1 & 0 & 0 \\
0 & 0 & 4 & 1 \\
0 & 0 & 1 & 4
\end{array}\right), L_{3} \cong\left(\begin{array}{cccc}
2 & -1 & 0 & 0 \\
-1 & 2 & 0 & 0 \\
0 & 0 & 2 & -1 \\
0 & 0 & -1 & 3
\end{array}\right) .
$$

All three lattices are reflective with the full-rank root systems

$$
\begin{aligned}
& R\left(L_{1}\right)={ }^{15} \mathrm{~B}_{1} \mathrm{~B}_{3}, \\
& R\left(L_{2}\right)={ }^{3} \mathrm{~A}_{1}{ }^{5} \mathrm{~A}_{1} \mathrm{~B}_{2}, \\
& R\left(L_{3}\right)=\mathrm{A}_{1}{ }^{5} \mathrm{~A}_{1} \mathrm{G}_{2} .
\end{aligned}
$$


Thus $\mathrm{I}\left(3^{-1} 5^{-1}\right)$ is totally-reflective and according to our structure theorem 4.3 the Lorentzian lattice $E:=\mathbb{H} \perp L_{1}$ is a candidate for a reflective lattice of signature $(5,1)$. Vinberg's algorithm has produced the following roots:

$$
\begin{array}{ll}
v_{1}=(0,0,-1,0,0,0), & v_{2}=(-1,-1,0,0,0,0), \\
v_{3}=(0,0,1,1,0,0), & v_{4}=(0,0,0,0,-1,1), \\
v_{5}=(0,0,0,0,1,1), & v_{6}=(0,1,1,-1,0,0), \\
v_{7}=(-1,1,0,0,0,-1), & v_{8}=(0,3,0,0,1,-1), \\
v_{9}=(-2,2,0,-2,1,-1), & v_{10}=(0,5,0,0,-1,-1), \\
v_{11}=(-2,3,0,-2,-1,-1), & v_{12}=(-10,10,5,-5,-4,-4), \\
v_{13}=(-10,10,5,-5,4,-6), & v_{14}=(-15,15,0,-15,2,-8), \\
v_{15}=(-15,15,0,-15,-2,-7) . &
\end{array}
$$

The polyhedron

$$
P_{15}:=\bigcap_{i=1}^{15} h_{v_{i}}^{+}
$$

has finite volume, which can easily be checked with lemma 3.21 . Then lemma 3.20 implies that $P_{15}$ is the fundamental domain of the hyperbolic reflection group $W(E)$,

$$
P(E)=P_{15}
$$

Finally, lemma 3.19 shows that $E$ is reflective, or equivalently, that $W(E)$ has finite index in $O^{+}(E)$.

For the next example consider the genus $\operatorname{II}\left(2_{6}^{+2} 3^{-3} 5^{-3}\right)$. It also contains three lattices,

$$
L_{1} \cong\left(\begin{array}{cccc}
4 & 1 & 0 & 0 \\
1 & 4 & 0 & 0 \\
0 & 0 & 30 & 0 \\
0 & 0 & 0 & 30
\end{array}\right), L_{2} \cong\left(\begin{array}{cccc}
10 & -5 & 0 & 0 \\
-5 & 10 & 0 & 0 \\
0 & 0 & 6 & 0 \\
0 & 0 & 0 & 30
\end{array}\right), L_{3} \cong\left(\begin{array}{cccc}
4 & 2 & -2 & -2 \\
2 & 16 & -1 & -1 \\
-2 & -1 & 16 & 1 \\
-2 & -1 & 1 & 16
\end{array}\right),
$$

with the full-rank root systems

$$
\begin{aligned}
& R\left(L_{1}\right)={ }^{3} \mathrm{~A}_{1}{ }^{5} \mathrm{~A}_{1}{ }^{30} \mathrm{~B}_{2}, \\
& R\left(L_{2}\right)={ }^{3} \mathrm{~A}_{1}{ }^{15} \mathrm{~A}_{1}{ }^{15} \mathrm{G}_{2}, \\
& R(L 3)={ }^{2} \mathrm{~A}_{1}{ }^{15} \mathrm{C}_{3} .
\end{aligned}
$$

Again, this genus is totally-reflective and $E:=\mathbb{H} \perp L_{1}$ is a candidate for a reflective lattice of signature $(5,1)$. In this case Vinberg's algorithm produces over 1300 fundamental roots in a short amount of time, thus one can expect this lattice to be non-reflective. The latter property can be proven rigorously with theorem 3.22 
if we can find a non-reflective orthogonal summand of $E$. Considering the Gram matrix of $L_{1}$, the 5-dimensional lattice

$$
\tilde{E}:=\mathbb{H} \perp \tilde{L}_{1}
$$

with $\tilde{L_{1}} \cong\left(\begin{array}{ccc}4 & 1 & 0 \\ 1 & 4 & 0 \\ 0 & 0 & 30\end{array}\right)$ is easily recognizable as a orthogonal summand of $E$. The classification result in Wal93 implies that $\tilde{E}$ is not a reflective Lorentzian lattice, thus neither is $E$.

For the last example consider the totally-reflective genus $\mathrm{II}\left(2_{\mathrm{II}}^{-2} 17^{+1}\right)$. It consists of two lattices

$$
L_{1} \cong\left(\begin{array}{cccc}
2 & 1 & 1 & -1 \\
1 & 2 & 1 & -1 \\
1 & 1 & 2 & 0 \\
-1 & -1 & 0 & 18
\end{array}\right), \quad L_{2} \cong\left(\begin{array}{cccc}
2 & 0 & 0 & -1 \\
0 & 2 & 0 & -1 \\
0 & 0 & 2 & -1 \\
-1 & -1 & -1 & 10
\end{array}\right)
$$

which have the root systems

$$
\begin{aligned}
& R\left(L_{1}\right)={ }^{34} \mathrm{~A}_{1} \mathrm{C}_{3}, \\
& R\left(L_{2}\right)={ }^{17} \mathrm{~A}_{1}{ }^{2} \mathrm{~B}_{3} .
\end{aligned}
$$

Vinberg's algorithm, applied to $E:=\mathbb{H} \perp L_{1}$, did not terminate after 100 hours of runtime, hence we expected $E$ to be non-reflective. Notice that both lattices are indecomposable, thus embedding smaller lattices does not seem to be promising. We will show that $E$ is not reflective by constructing an symmetry of infinit order of $P(E)$ using theorem 3.24 . It then follows from

$$
O^{+}(E)=W(E) \rtimes H
$$

with $H:=\operatorname{Sym}(P(E)) \cap O^{+}(E)$ that $W(E)$ is not of finite index in $O^{+}(E)$ (cf. lemma 3.17). The following set of fundamental roots of $E$ satisfies the condition of theorem 3.24 .

$$
\begin{array}{ll}
v_{1}=(0,0,-1,0,1,0), & v_{2}=(0,0,1,-1,1,0), \\
v_{3}=(0,0,0,1,-1,0), & v_{4}=(-1,0,0,-1,0,0), \\
v_{5}=(-4,2,0,0,-1,-1), & v_{6}=(-8,1,1,0,-1,-1), \\
v_{7}=(-11,3,1,1,-1,-2) . &
\end{array}
$$

The first condition requires the roots to generate $E$, which can be checked with standard routines in MAGMA. Regarding the second condition, we recognize

$$
h_{v_{1}}^{+} \cap h_{v_{2}}^{+} \cap h_{v_{3}}^{+} \cap h_{v_{6}}^{+} \cap h_{v_{7}}^{+}
$$


as a vertex since the normalized Gram matrix of the system $\left(v_{1}, v_{2}, v_{3}, v_{6}, v_{7}\right)$,

$$
\left(\begin{array}{ccccc}
1 & 0 & -\cos \left(\frac{\pi}{3}\right) & -\cos \left(\frac{\pi}{3}\right) & 0 \\
0 & 1 & -\cos \left(\frac{\pi}{4}\right) & 0 & 0 \\
-\cos \left(\frac{\pi}{3}\right) & -\cos \left(\frac{\pi}{4}\right) & 1 & 0 & 0 \\
-\cos \left(\frac{\pi}{3}\right) & 0 & 0 & 1 & -\cos \left(\frac{\pi}{3}\right) \\
0 & 0 & 0 & -\cos \left(\frac{\pi}{3}\right) & 1
\end{array}\right)
$$

is positive-definite (this is sufficient according to theorem 3.9). Let $\Gamma_{7}$ be the Coxeter diagram of the vector system $\left(v_{1}, v_{2}, v_{3}, v_{4}, v_{5}, v_{6}, v_{7}\right)$,

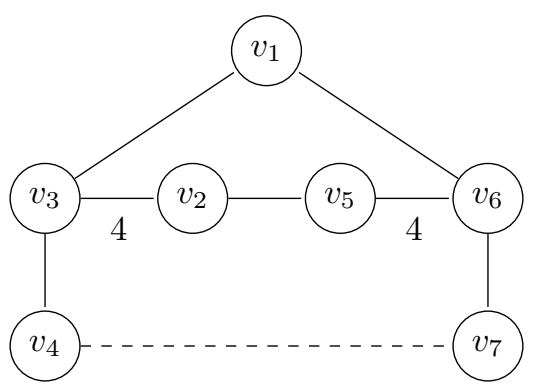

Theorem 3.24 implies that every non-trivial graph automorphism induces an nontrivial symmetry from $\operatorname{Sym}(P(E)) \cap O^{+}(E)$. As can be seen above, the permutation

$$
(2,5)(3,6)(4,7)
$$

is a graph automorphism of $\Gamma_{7}$ and the symmetry $\varphi$ that permutes the corresponding $v_{i}$ has infinite order. The latter statement follows from lemma 3.23 the set of fixed vectors of $\varphi$ is the one-dimensional subspace of $E \otimes \mathbb{Q}$ generated by $\left(1,-\frac{1}{2},-\frac{1}{4}, \frac{1}{4}, 0, \frac{1}{4}\right)$. Since this generator is of length $\frac{1}{4}$, the subspace has an empty intersection with the light cone (the set of all negative-norm vectors) and thus the subgroup of $\operatorname{Sym}(P)$ generated by $\varphi$ has infinite cardinality. 



\section{Index}

arithmetic hyperbolic reflection group, 38

canonical Jordan decomposition, 8

class number, 8

compartment, 8

conformal ball model, 34

controlling vector, 40

Coxeter diagram, 37

Coxeter polyhedron, 35

determinant, 7

discriminant group, 7

dual lattice, 7

Dynkin diagram, 13

even lattice, 7

fundamental domain, 35

fundamental polyhedron, 35

fundamental roots, 39

genus, 8

genus symbol, 9

glue code, 14

half-space, 35

hyperbolic $n$-space, 33

hyperbolic distance, 33

hyperbolic plane, 45

hyperbolic reflection, 34

hyperbolic reflection group, 34

hyperplane in $H^{n}, 34$

integral lattice, 7

interval of a Jordan decomposition, 8

isometry group of $H^{n}, 34$
Jordan decomposition, 8

lattice, 7

Liouville function, 10

localization, 8

Lorentzian lattice, 38

mass, 9

maximal hyperbolic reflection group, 39

modular lattice, 7

normalized Gram matrix, 36

odd lattice, 7

oddity of a compartment, 8

polyhedron, 35

primitive lattice, 7

reflective Lorentzian lattice, 39

reflective positive-definite lattice, 13

root, 12

root system, 12

square-free lattice, 8

standard mass, 10

strongly square-free lattice, 8

totally-reflective, 16

unimodular lattice, 7

Vinberg's algorithm, 40

Vinbergs's lemma, 45

Weyl group, 12 



\section{Bibliography}

[All12] D. Allcock, The reflective Lorentzian lattices of rank 3, Mem. Am. Math. Soc. 1033 (2012), iii-ix +108 .

[And71a] E.M. Andreev, On convex polyhedra in Lobachevskij spaces, Math. USSR, Sb. 10 (1971), $413-440$.

[And71b] _ On convex polyhedra of finite volume in Lobachevskii space, Math. USSR, Sb. 12 (1971), 255-259.

[BCP97] W. Bosma, J. Cannon, and C. Playoust, The Magma algebra system. I. The user language, J. Symbolic Comput. 24 (1997), no. 3-4, 235-265, Computational algebra and number theory (London, 1993).

[Ber93] W. Berger, Geschlechter stark quadratfreier reflektiver Gitter, Diploma thesis, Univ. Bielefeld, 1993.

[BH62] A. Borel and Harish-Chandra, Arithmetic subgroups of algebraic groups, Ann. Math. (2) 75 (1962), 485-535.

[Bie81] J. Biermann, Gitter mit kleiner Automorphismengruppe in Geschlechtern von $\mathbb{Z}$-Gittern mit positiv-definiter quadratischer Form, Ph.D. thesis, Univ. Göttingen, 1981.

[BL14] M. Belolipetsky and B. Linowitz, On fields of definition of arithmetic Kleinian reflection groups. II, Int. Math. Res. Not. 2014 (2014), no. 9, 2559-2571.

[Bor87] R. Borcherds, Automorphism groups of Lorentzian lattices, J. Algebra 111 (1987), 133153.

[Bou08] N. Bourbaki, Elements of mathematics. Lie groups and Lie algebras. Chapters 4-6. Transl. from the French by Andrew Pressley. Paperback reprint of the hardback edition 2002, Berlin: Springer, 2008.

[Bug84] V.O. Bugaenko, Groups of automorphisms of unimodular hyperbolic quadratic forms over the ring $\mathbb{Z}[(\sqrt{5}+1) / 2]$, Mosc. Univ. Math. Bull. 39 (1984), no. 5.

[Bug90] - On reflective unimodular hyperbolic quadratic forms, Sel. Math. Sov. 9 (1990), no. $3,263-271$.

[Bug92] _ Arithmetic crystallographic groups generated by reflections, and reflective hyperbolic lattices, Lie groups, their discrete subgroups, and invariant theory, 1992, pp. 3355.

[Cox34] H.S.M. Coxeter, Discrete groups generated by reflections, Ann. Math. (2) 35 (1934), 588-621.

[CS88] J.H. Conway and N.J.A. Sloane, Low-dimensional lattices. IV: The mass formula, Proc. R. Soc. Lond., Ser. A 419 (1988), 259-286.

[CS99] - Sphere packings, lattices and groups, 3rd ed., New York, NY: Springer, 1999.

[Ess96] F. Esselmann, Über die maximale Dimension von Lorentz-Gittern mit coendlicher Spiegelungsgruppe, Journal of Number Theory 61 (1996), no. 1, 103-144.

[Kap74] I.M. Kaplinskaya, Discrete groups generated by reflections in the faces of simplicial prisms in Lobachevskian spaces, Math. Notes 15 (1974), 88-91. 
[Kit93] Y. Kitaoka, Arithmetic of quadratic forms, Springer, 1993.

[Kle79] F. Klein, Ueber die Transformation siebenter Ordnung der elliptischen Functionen, Math. Ann. 14 (1879), 428-471.

[Kne56] M. Kneser, Klassenzahlen indefiniter quadratischer Formen in drei oder mehr Veränderlichen, Arch. Math. 7 (1956), 323-332.

[Kne57] _ Klassenzahlen definiter quadratischer Formen, Arch. Math. 8 (1957), 241-250.

[Kne02] _ Quadratische formen, Springer, 2002.

[Leh60] R.S. Lehman, On Liouville's function, Math. Comput. 14 (1960), 311-320.

[LK13] D. Lorch and M. Kirschmer, Single-class genera of positive integral lattices, LMS J. Comput. Math. 16 (2013), 172-186.

[Mac11] C. Maclachlan, Bounds for discrete hyperbolic arithmetic reflection groups in dimension 2, Bull. Lond. Math. Soc. 43 (2011), no. 1, 111-123.

[Mak70] V.S. Makarov, On Fedorov groups of four- and five-dimensional Lobachevsky spaces, Studies in general algebra, Kishinev State Univ., 1970, Russian, pp. 120-129.

[Mar15] A. Mark, The classification of rank 3 reflective hyperbolic lattices over $\mathbb{Z}[\sqrt{2}]$, Ph.D. thesis, University of Texas at Austin, 2015.

[Nik81] V.V. Nikulin, On arithmetic groups generated by reflections in Lobachevsky spaces, Math. USSR, Izv. 16 (1981), 573-601.

[Nik82] - On the classification of arithmetic groups generated by reflections in Lobachevsky spaces, Math. USSR, Izv. 18 (1982), 99-123.

[Nik00] - On the classification of hyperbolic root systems of rank three. Transl. from the Russian, Moscow: Maik Nauka/Interperiodica, 2000.

[Nik09] - On ground fields of arithmetic hyperbolic reflection groups, Groups and symmetries. From Neolithic Scots to John McKay. Selected papers of the conference, Montreal, Canada, April 27-29, 2007., 2009, pp. 299-326.

[Nik11] The transition constant for arithmetic hyperbolic reflection groups, Izv. Math. 75 (2011), no. 5, 971-1005.

[O'M00] O. T. O'Meara, Introduction to quadratic forms, reprint of the 1973 edition ed., Berlin: Springer, 2000.

[Poi82] H. Poincaré, Théorie des groupes fuchsiens, Acta Math. 1 (1882), 1-62.

[Pro86] M.N. Prokhorov, The absence of discrete groups of reflections with noncompact fundamental polyhedron of finite volume in Lobachevskij space of large dimension, Izv. Akad. Nauk SSSR, Ser. Mat. 50 (1986), no. 2, 413-424.

[Rat06] J.G. Ratcliffe, Foundations of hyperbolic manifolds, 2nd ed., NY: Springer, 2006.

[SB96] R. Scharlau and B. Blaschke, Reflective integral lattices, J. Algebra 181 (1996), no. 3, 934-961.

[Sch89] R. Scharlau, On the classification of arithmetic reflection groups in hyperbolic 3-space, Preprint, 1989.

[SH98] R. Scharlau and B. Hemkemeier, Classification of integral lattices with large class number, Math. Comput. 67 (1998), no. 222, 737-749.

[Sie35] C.L. Siegel, Über die analytische Theorie der quadratischen Formen, Ann. Math. (2) 36 (1935), 527-606.

[SW92] R. Scharlau and C. Walhorn, Integral lattices and hyperbolic reflection groups, Journées arithmétiques. Exposés présentés aux dix-septièmes congrès à Genève, Suisse, 9-13 septembre 1991, 1992, pp. 279-291. 
[Tit66] J. Tits, Classification of algebraic semisimple groups, Proc. Symp. Pure Math. 9, 33-62 (1966), 1966.

[Vin67] E.B. Vinberg, Discrete groups generated by reflections in Lobachevskii spaces, Mat. Sb., Nov. Ser. 72 (1967), 471-488.

[Vin72a] - On groups of unit elements of certain quadratic forms, Math. USSR, Sb. 16 (1972), 17-35.

[Vin72b] - On unimodular integral quadratic forms, Funct. Anal. Appl. 6 (1972), 105-111.

[Vin81] - Absence of crystallographic reflection groups in Lobachevskij spaces of large dimension, Funkts. Anal. Prilozh. 15 (1981), no. 2, 67-68.

[Vin84] - The non-exitence of crystallographic groups of reflections in Lobachevskij spaces of large dimension, Trans. Mosc. Math. Soc. 1985 (1984), 75-112.

[Vin85] _ Hyperbolic reflection groups, Russ. Math. Surv. 40 (1985), no. 1, 31-75.

[VK78] E.B. Vinberg and I.M. Kaplinskaya, On the groups $O_{18,1}(\mathbb{Z})$ and $O_{19,1}(\mathbb{Z})$, Sov. Math., Dokl. 19 (1978), 194-197.

[Wal93] C. Walhorn, Arithmetische Spiegelungsgruppen auf dem 4-dimensionalen hyperbolischen Raum, Ph.D. thesis, Univ. Bielefeld, 1993.

[Wat62] G.L. Watson, Transformations of a quadratic form which do not increase the classnumber, Proc. Lond. Math. Soc. (3) 12 (1962), 577-587.

[Wat73] - Transformations of a quadratic form which do not increase the class-number. II, Acta Arith. 27 (1973), 171-189.

[Wat79] _ Existence of an indecomposable positive quadratic form in a given genus of rank at least 14, Acta Arith. 35 (1979), 55-100. 



\section{APPENDIX A}

\section{Description of Some MAGMA Functions}

- DpGen(g) : SeqEnum -> SeqEnum

Calculates the partial dual $D_{p_{i_{1}} \ldots p_{i_{k}}}(g)$ of the genus symbol $g$ for every square-free divisor of det $g$.

- FindLatticeWithGenusSymbol(g) : SeqEnum -> Lat

Calculates a representative lattice for the genus $g$. This function was implemented by Lorch \& Kirschmer (cf. [LK13, section 5.2).

- FindTotallyReflectiveHelp(L,p) : Lat, RngIntElt -> FldReElt

Calculates the quantity of corollary 2.16 needed in the process of finding all totally-reflective genera.

- FindTotallyReflective( $(T)$ : SeqEnum -> SetEnum

Starting with the list $T$ of all square-free totally-reflective genera in dimension 4 , this function calculates all totally-reflective genera in dimension 4 . This function uses WatsonPrelmages (see below) with respect to primes given by FindTotallyReflectiveHelp to search for totally-reflective lattices in the pre-images (of lattices which are already known to be totally-reflective) under the Watson transformation.

- FundamentalRoots(G) : AlgMatElt -> SeqEnum

Let $L$ be a lattice with Gram matrix $G$. If $G$ is positive-definite, then this function calculates a root basis of the root system $R(L)$. If $G$ has signature $(n, 1)$, then this function calculates a root basis of the root system $\left\{v \in R(L) \mid\left(v, p_{0}\right)=0\right\}$, where $p_{0}$ is the controlling vector of Vinberg's algorithm.

- FundamentalRootsHyp(G) : AlgMatElt -> SeqEnum

This is our MAGMA implementation of Vinberg's algorithm. Let $E=$ ${ }^{\alpha} \mathbb{H} \perp L$ be a Lorentzian lattice with $L$ positive-definite, $\operatorname{dim} L=4$ and Gram-Matrix $G$. Using the controlling vector $p_{0}=(1,-1,0,0,0,0)$, this function will calculates all fundamental roots of $E$.

- GenusSymbols(G) : AlgMatElt -> SeqEnum

Calculates the genus symbol of the lattice with Gram matrix $G$. This function was implemented by Lorch (cf. [LK13]). 
- HasFiniteVolume5(G,FR) : AlgMatElt, SeqEnum -> List

Let $E$ be the Lorentzian lattice of signature $(5,1)$ with Gram matrix $G$. This function checks if the polyhedron spanned by the fundamental roots of $E$ that are contained in FR is finite and calculates its combinatorial structure.

- InfiniteOrderSymmetry(G,FR) : AlgMatElt, SeqEnum -> BoolElt

This function searches for subgraphs of the Coxeter diagram belonging to the roots in FR such that the requirements of theorem 3.24 are satisfied. If such subgraph is found, then the non-trivial graph automorphism is transformed into a symmetry of the fundamental polyhedron of the lattice with Gram matrix $G$ and it is checked, whether its fixed vectors have empty intersection with the light cone.

- IsGenusSymbol(g) : SeqEnum -> BoolElt

Checks if there exists a lattice $L$ with the genus symbol $g$.

- IsPositiveDefiniteHyp $(G)$ : AlgMatElt -> BoolElt

Checks if the matrix $G$ is positive-definite. The entries of $G$ has to be given in floating-point arithmetic.

- IsPositiveSemiDefiniteHyp(G) : AlgMatElt -> BoolElt

Checks if the matrix $G$ is positive-semidefinite. The entries of $G$ has to be given in floating-point arithmetic.

- IsTotallyReflective(L,k) : Lat, RngIntElt -> BoolElt

Checks if the lattice $L$ is totally-reflective. The parameter $k \in\{1,2,3,4\}$ decides how much of the genus of $L$ will be calculated during the process. If $k=1$, then only $L$ is checked for reflectivity. If $k=2$, then all neighbours of $L$ with respect to (legitimate) primes $2 \leq p \leq 19$ are checked for reflectivity. The process of calculating all isometry classes in the neighbourhood of $L$ with respect to $p$ can be very time consuming. This is when the following two parameters come into play. If $k=3$, then single neighbours of $L$ with respect to a (legitimate) neighbour-vector $v_{p}$ and a (legitimate) primes $2 \leq p \leq 499$ are checked for reflectivity. Here, the neighbour-vectors are considered in the order given by MAGMA's routine ShortVectors. If $k=4$, then the same is done as for $k=3$ with the only exception that the neighbour-vector is chosen randomly in ShortVectors.

- MassRational(L) : Lat -> FldRatElt

Calculates the mass of the lattice $L$.

- MassVgl(G) : AlgMatElt -> FldReElt

Calculates the ratio $\mathrm{M}_{\text {ref }} / \mathrm{M}$ for a 4-dimensional lattice $L$ with Gram matrix $G$. 
- PartialRootSystemHyp(G,a,i) : AlgMatElt, RngIntElt, RngIntElt -> SeqEnum Calculates all roots $v$ of the Lorentzian lattice with Gram matrix $G$ such that $(v, v)=a$ and $\left(v, p_{0}\right)=i$, where $p_{0}$ is the controlling vector of Vinberg's algorithm.

- RootSystemPos(G) : AlgMatElt -> BoolElt

Checks if the positive-definite lattice $L$ with Gram matrix $G$ is reflective.

It can also be used to calculate all roots of $L$, the root basis of $R(L)$ and the combinatorial class of $R(L)$.

- WatsonPrelmages(G,p) : AlgMatElt, RngIntElt -> SeqEnum

Calculates the pre-images of the genus of $L$, where $L$ is a 4-dimensional lattice with Gram matrix $G$, under the Watson transformation with respect to the prime $p$. 

APPENDIX B

\section{Primitive Totally-Reflective Genera of Dimension}

\section{3}

\begin{tabular}{|c|c|c|c|c|c|c|c|c|}
\hline det & Genus & $\mathrm{h}$ & det & Genus & $\mathrm{h}$ & $\operatorname{det}$ & Genus & $\mathrm{h}$ \\
\hline 1 & $\mathrm{I}\left(1_{3}^{+3}\right)$ & 1 & 2 & $\mathrm{I}\left(2_{1}^{+1}\right)$ & 1 & 3 & $\mathrm{I}\left(3^{-1}\right)$ & 1 \\
\hline 3 & $\mathrm{I}\left(3^{+1}\right)$ & 1 & 4 & $\mathrm{I}\left(2_{2}^{+2}\right)$ & 1 & 4 & $\mathrm{II}\left(4_{3}^{-1}\right)$ & 1 \\
\hline 4 & $\mathrm{I}\left(4_{1}^{+1}\right)$ & 1 & 5 & $\mathrm{I}\left(5^{-1}\right)$ & 1 & 5 & $\mathrm{I}\left(5^{+1}\right)$ & 1 \\
\hline 6 & $\operatorname{II}\left(2_{1}^{+1} 3^{-1}\right)$ & 1 & 6 & $\mathrm{I}\left(2_{1}^{+1} 3^{-1}\right)$ & 1 & 6 & $\mathrm{I}\left(2_{1}^{+1} 3^{+1}\right)$ & 1 \\
\hline 7 & $\mathrm{I}\left(7^{+1}\right)$ & 2 & 8 & $\mathrm{I}\left(2_{1}^{+1} 4_{1}^{+1}\right)$ & 1 & 9 & $\mathrm{I}\left(3^{-2}\right)$ & 1 \\
\hline 9 & $\mathrm{I}\left(9^{-1}\right)$ & 1 & 9 & $\mathrm{I}\left(9^{+1}\right)$ & 1 & 9 & $\mathrm{I}\left(3^{+2}\right)$ & 1 \\
\hline 10 & $\mathrm{I}\left(2_{1}^{+1} 5^{-1}\right)$ & 2 & 10 & $\mathrm{I}\left(2_{1}^{+1} 5^{+1}\right)$ & 1 & 11 & $\mathrm{I}\left(11^{-1}\right)$ & 1 \\
\hline 12 & $\mathrm{I}\left(2_{2}^{+2} 3^{+1}\right)$ & 1 & 12 & $\mathrm{I}\left(2_{\mathrm{II}}^{+2} 3^{+1}\right)$ & 1 & 12 & $\mathrm{I}\left(2_{2}^{+2} 3^{-1}\right)$ & 1 \\
\hline 12 & $\mathrm{II}\left(4_{1}^{+1} 3^{-1}\right)$ & 1 & 12 & $\mathrm{I}\left(4_{7}^{+1} 3^{-1}\right)$ & 1 & 12 & $\mathrm{I}\left(4_{1}^{+1} 3^{+1}\right)$ & 1 \\
\hline 12 & $\mathrm{I}\left(4_{7}^{+1} 3^{+1}\right)$ & 1 & 12 & $\operatorname{II}\left(4_{5}^{-1} 3^{+1}\right)$ & 1 & 14 & $\mathrm{II}\left(2_{1}^{+1} 7^{+1}\right)$ & 1 \\
\hline 14 & $\mathrm{I}\left(2_{1}^{+1} 7^{+1}\right)$ & 2 & 15 & $\mathrm{I}\left(3^{+1} 5^{+1}\right)$ & 2 & 15 & $\mathrm{I}\left(3^{-1} 5^{-1}\right)$ & 2 \\
\hline 15 & $\mathrm{I}\left(3^{+1} 5^{-1}\right)$ & 1 & 15 & $\mathrm{I}\left(3^{-1} 5^{+1}\right)$ & 1 & 16 & $\mathrm{I}\left(4_{\mathrm{II}}^{-2}\right)$ & 1 \\
\hline 16 & $\mathrm{I}\left(4_{2}^{+2}\right)$ & 1 & 16 & $\mathrm{I}\left(16_{5}^{-1}\right)$ & 1 & 16 & $\mathrm{I}\left(16_{1}^{+1}\right)$ & 2 \\
\hline 17 & $\mathrm{I}\left(17^{+1}\right)$ & 2 & 18 & $\mathrm{II}\left(2_{7}^{+1} 3^{+2}\right)$ & 1 & 18 & $\mathrm{I}\left(2_{1}^{+1} 3^{-2}\right)$ & 1 \\
\hline 18 & $\mathrm{I}\left(2_{1}^{+1} 9^{-1}\right)$ & 2 & 18 & $\mathrm{I}\left(2_{1}^{+1} 3^{+2}\right)$ & 1 & 20 & $\mathrm{I}\left(2_{2}^{+2} 5^{-1}\right)$ & 1 \\
\hline 20 & $\mathrm{I}\left(2_{2}^{+2} 5^{+1}\right)$ & 2 & 20 & $\operatorname{II}\left(4_{7}^{+1} 5^{+1}\right)$ & 1 & 20 & $\mathrm{I}\left(4_{1}^{+1} 5^{-1}\right)$ & 1 \\
\hline 20 & $\mathrm{I}\left(4_{1}^{+1} 5^{+1}\right)$ & 2 & 20 & $\operatorname{II}\left(4_{3}^{-1} 5^{-1}\right)$ & 1 & 21 & $\mathrm{I}\left(3^{+1} 7^{+1}\right)$ & 2 \\
\hline 21 & $\mathrm{I}\left(3^{-1} 7^{+1}\right)$ & 2 & 21 & $\mathrm{I}\left(3^{+1} 7^{-1}\right)$ & 1 & 24 & $\mathrm{I}\left(2_{1}^{+1} 4_{1}^{+1} 3^{+1}\right)$ & 2 \\
\hline 24 & $\mathrm{I}\left(2_{1}^{+1} 4_{1}^{+1} 3^{-1}\right)$ & 1 & 25 & $\mathrm{I}\left(5^{+2}\right)$ & 1 & 25 & $\mathrm{I}\left(5^{-2}\right)$ & 1 \\
\hline 27 & $\mathrm{I}\left(3^{-1} 9^{+1}\right)$ & 1 & 27 & $\mathrm{I}\left(3^{-1} 9^{-1}\right)$ & 1 & 27 & $\mathrm{I}\left(3^{+1} 9^{-1}\right)$ & 1 \\
\hline 27 & $\mathrm{I}\left(3^{+1} 9^{+1}\right)$ & 1 & 28 & $\mathrm{I}\left(2_{\mathrm{II}}^{+2} 7^{+1}\right)$ & 1 & 28 & $\mathrm{I}\left(2_{2}^{+2} 7^{+1}\right)$ & 2 \\
\hline 28 & $\mathrm{II}\left(4_{1}^{+1} 7^{+1}\right)$ & 2 & 28 & $\mathrm{I}\left(4_{7}^{+1} 7^{+1}\right)$ & 2 & 30 & $\mathrm{II}\left(2_{1}^{+1} 3^{-1} 5^{-1}\right)$ & 2 \\
\hline 30 & $\mathrm{II}\left(2_{1}^{+1} 3^{+1} 5^{+1}\right)$ & 1 & 30 & $\mathrm{I}\left(2_{1}^{+1} 3^{-1} 5^{-1}\right)$ & 2 & 30 & $\mathrm{I}\left(2_{1}^{+1} 3^{+1} 5^{+1}\right)$ & 2 \\
\hline 30 & $\mathrm{I}\left(2_{1}^{+1} 3^{+1} 5^{-1}\right)$ & 1 & 32 & $\mathrm{I}\left(2_{1}^{+1} 16_{5}^{-1}\right)$ & 1 & 32 & $\mathrm{I}\left(2_{1}^{+1} 16_{3}^{-1}\right)$ & 1 \\
\hline 32 & $\mathrm{I}\left(2_{1}^{+1} 16_{1}^{+1}\right)$ & 1 & 32 & $\mathrm{I}\left(2_{1}^{+1} 16_{7}^{+1}\right)$ & 1 & 33 & $\mathrm{I}\left(3^{+1} 11^{-1}\right)$ & 2 \\
\hline 33 & $\mathrm{I}\left(3^{-1} 11^{+1}\right)$ & 2 & 35 & $\mathrm{I}\left(5^{+1} 7^{-1}\right)$ & 1 & 35 & $\mathrm{I}\left(5^{-1} 7^{+1}\right)$ & 2 \\
\hline 36 & $\mathrm{I}\left(2_{2}^{+2} 3^{-2}\right)$ & 1 & 36 & $\mathrm{I}\left(2_{2}^{+2} 9^{+1}\right)$ & 2 & 36 & $\mathrm{I}\left(2_{2}^{+2} 3^{+2}\right)$ & 1 \\
\hline
\end{tabular}




\begin{tabular}{|c|c|c|c|c|c|c|c|}
\hline 36 & $\mathrm{I}\left(2_{\mathrm{II}}^{+2} 3^{+2}\right)$ & 1 & 36 & $\mathrm{II}\left(4_{3}^{-1} 3^{-2}\right)$ & 1 & 36 & $\operatorname{II}\left(4_{3}^{-1} 9^{-1}\right)$ \\
\hline 36 & $\mathrm{II}\left(4_{3}^{-1} 9^{+1}\right)$ & 1 & 36 & $\mathrm{II}\left(4_{7}^{+1} 3^{+2}\right)$ & 1 & 36 & $\mathrm{I}\left(4_{7}^{+1} 3^{+2}\right)$ \\
\hline 36 & $\mathrm{I}\left(4_{1}^{+1} 3^{-2}\right)$ & 1 & 36 & $\mathrm{I}\left(4_{1}^{+1} 9^{+1}\right)$ & 2 & 36 & $\mathrm{I}\left(4_{1}^{+1} 3^{+2}\right)$ \\
\hline 39 & $\mathrm{I}\left(3^{+1} 13^{-1}\right)$ & 1 & 40 & $\mathrm{I}\left(2_{1}^{+1} 4_{1}^{+1} 5^{+1}\right)$ & 2 & 42 & $\operatorname{II}\left(2_{7}^{+1} 3^{-1} 7^{+1}\right)$ \\
\hline 42 & $\mathrm{I}\left(2_{1}^{+1} 3^{-1} 7^{-1}\right)$ & 2 & 42 & $\mathrm{I}\left(2_{1}^{+1} 3^{-1} 7^{+1}\right)$ & 2 & 44 & $\operatorname{II}\left(4_{1}^{+1} 11^{-1}\right)$ \\
\hline 45 & $\mathrm{I}\left(3^{+2} 5^{+1}\right)$ & 2 & 45 & $\mathrm{I}\left(3^{-2} 5^{-1}\right)$ & 2 & 45 & $\mathrm{I}\left(3^{+2} 5^{-1}\right)$ \\
\hline 45 & $\mathrm{I}\left(3^{-2} 5^{+1}\right)$ & 1 & 45 & $\mathrm{I}\left(9^{-1} 5^{+1}\right)$ & 2 & 48 & $\mathrm{I}\left(4_{2}^{+2} 3^{+1}\right)$ \\
\hline 48 & $\mathrm{I}\left(4_{6}^{+2} 3^{-1}\right)$ & 1 & 48 & $\mathrm{I}\left(4_{\mathrm{II}}^{+2} 3^{+1}\right)$ & 1 & 48 & $\mathrm{I}\left(4_{0}^{+2} 3^{+1}\right)$ \\
\hline 48 & $\mathrm{I}\left(4_{\mathrm{II}}^{-2} 3^{-1}\right)$ & 1 & 48 & $\operatorname{II}\left(16_{1}^{+1} 3^{-1}\right)$ & 2 & 48 & $\mathrm{I}\left(16_{1}^{+1} 3^{+1}\right)$ \\
\hline 48 & $\mathrm{I}\left(16_{7}^{+1} 3^{+1}\right)$ & 1 & 48 & $\mathrm{I}\left(16_{5}^{-1} 3^{+1}\right)$ & 2 & 48 & $\mathrm{I}\left(16_{3}^{-1} 3^{+1}\right)$ \\
\hline 49 & $\mathrm{I}\left(7^{+2}\right)$ & 2 & 50 & $\mathrm{I}\left(2_{1}^{+1} 5^{+2}\right)$ & 2 & 50 & $\mathrm{I}\left(2_{1}^{+1} 5^{-2}\right)$ \\
\hline 51 & $\mathrm{I}\left(3^{-1} 17^{+1}\right)$ & 2 & 54 & $\mathrm{II}\left(2_{1}^{+1} 3^{-1} 9^{-1}\right)$ & 2 & 54 & $\mathrm{I}\left(2_{1}^{+1} 3^{-1} 9^{-1}\right)$ \\
\hline 54 & $\mathrm{I}\left(2_{1}^{+1} 3^{-1} 9^{+1}\right)$ & 1 & 54 & $\mathrm{I}\left(2_{1}^{+1} 3^{+1} 9^{+1}\right)$ & 1 & 54 & $\mathrm{I}\left(2_{1}^{+1} 3^{+1} 9^{-1}\right)$ \\
\hline 56 & $\mathrm{I}\left(2_{1}^{+1} 4_{1}^{+1} 7^{+1}\right)$ & 3 & 57 & $\mathrm{I}\left(3^{+1} 19^{-1}\right)$ & 2 & 60 & $\mathrm{I}\left(2_{\mathrm{II}}^{+2} 3^{+1} 5^{+1}\right)$ \\
\hline 60 & $\mathrm{I}\left(2_{\mathrm{II}}^{+2} 3^{-1} 5^{-1}\right)$ & 1 & 60 & $\mathrm{I}\left(2_{2}^{+2} 3^{-1} 5^{+1}\right)$ & 1 & 60 & $\mathrm{I}\left(2_{2}^{+2} 3^{+1} 5^{+1}\right)$ \\
\hline 60 & $\mathrm{I}\left(2_{2}^{+2} 3^{-1} 5^{-1}\right)$ & 2 & 60 & $\mathrm{II}\left(4_{5}^{-1} 3^{+1} 5^{-1}\right)$ & 1 & 60 & $\mathrm{II}\left(4_{5}^{-1} 3^{-1} 5^{+1}\right)$ \\
\hline 60 & $\mathrm{II}\left(4_{1}^{+1} 3^{+1} 5^{+1}\right)$ & 2 & 60 & $\mathrm{II}\left(4_{1}^{+1} 3^{-1} 5^{-1}\right)$ & 2 & 60 & $\mathrm{I}\left(4_{7}^{+1} 3^{+1} 5^{+1}\right)$ \\
\hline 60 & $\mathrm{I}\left(4_{7}^{+1} 3^{-1} 5^{-1}\right)$ & 2 & 60 & $\mathrm{I}\left(4_{7}^{+1} 3^{-1} 5^{+1}\right)$ & 1 & 63 & $\mathrm{I}\left(3^{+2} 7^{-1}\right)$ \\
\hline 63 & $\mathrm{I}\left(3^{-2} 7^{-1}\right)$ & 2 & 63 & $\mathrm{I}\left(3^{+2} 7^{+1}\right)$ & 1 & 64 & $\mathrm{I}\left(4_{1}^{+1} 16_{1}^{+1}\right)$ \\
\hline 65 & $\mathrm{I}\left(5^{+1} 13^{+1}\right)$ & 2 & 66 & $\mathrm{I}\left(2_{1}^{+1} 3^{+1} 11^{-1}\right)$ & 3 & 68 & $\mathrm{II}\left(4_{3}^{-1} 17^{+1}\right)$ \\
\hline 69 & $\mathrm{I}\left(3^{-1} 23^{+1}\right)$ & 3 & 72 & $\mathrm{I}\left(2_{1}^{+1} 4_{1}^{+1} 3^{-2}\right)$ & 2 & 72 & $\mathrm{I}\left(2_{1}^{+1} 4_{1}^{+1} 3^{+2}\right)$ \\
\hline 75 & $\mathrm{I}\left(3^{-1} 5^{+2}\right)$ & 1 & 75 & $\mathrm{I}\left(3^{+1} 5^{-2}\right)$ & 1 & 75 & $\mathrm{I}\left(3^{-1} 5^{-2}\right)$ \\
\hline 75 & $\mathrm{I}\left(3^{+1} 5^{+2}\right)$ & 2 & 77 & $\mathrm{I}\left(7^{+1} 11^{-1}\right)$ & 3 & 78 & $\operatorname{II}\left(2_{1}^{+1} 3^{-1} 13^{-1}\right)$ \\
\hline 80 & $\mathrm{I}\left(4_{\mathrm{II}}^{+2} 5^{-1}\right)$ & 1 & 80 & $\mathrm{I}\left(4_{2}^{+2} 5^{+1}\right)$ & 2 & 80 & $\mathrm{I}\left(4_{6}^{+2} 5^{-1}\right)$ \\
\hline 80 & $\mathrm{I}\left(4_{\mathrm{II}}^{-2} 5^{+1}\right)$ & 1 & 80 & $\mathrm{I}\left(16_{1}^{+1} 5^{-1}\right)$ & 2 & 80 & $\mathrm{I}\left(16_{1}^{+1} 5^{+1}\right)$ \\
\hline 80 & $\mathrm{I}\left(16_{5}^{-1} 5^{+1}\right)$ & 4 & 81 & $\mathrm{I}\left(3^{+1} 27^{-1}\right)$ & 1 & 81 & $\mathrm{I}\left(9^{-2}\right)$ \\
\hline 81 & $\mathrm{I}\left(9^{+2}\right)$ & 1 & 84 & $\mathrm{I}\left(2_{2}^{+2} 3^{+1} 7^{+1}\right)$ & 2 & 84 & $\mathrm{I}\left(2_{\mathrm{II}}^{+2} 3^{+1} 7^{+1}\right)$ \\
\hline 84 & $\mathrm{I}\left(2_{2}^{+2} 3^{+1} 7^{-1}\right)$ & 2 & 84 & $\mathrm{II}\left(4_{7}^{+1} 3^{-1} 7^{+1}\right)$ & 2 & 84 & $\operatorname{II}\left(4_{7}^{+1} 3^{+1} 7^{-1}\right)$ \\
\hline 84 & $\mathrm{I}\left(4_{1}^{+1} 3^{+1} 7^{+1}\right)$ & 2 & 84 & $\mathrm{I}\left(4_{1}^{+1} 3^{+1} 7^{-1}\right)$ & 2 & 84 & $\operatorname{II}\left(4_{3}^{-1} 3^{+1} 7^{+1}\right)$ \\
\hline 90 & $\operatorname{II}\left(2_{7}^{+1} 3^{-2} 5^{+1}\right)$ & 2 & 90 & $\operatorname{II}\left(2_{7}^{+1} 3^{+2} 5^{-1}\right)$ & 1 & 90 & $\mathrm{I}\left(2_{1}^{+1} 3^{+2} 5^{+1}\right)$ \\
\hline 90 & $\mathrm{I}\left(2_{1}^{+1} 3^{-2} 5^{+1}\right)$ & 2 & 90 & $\mathrm{I}\left(2_{1}^{+1} 3^{+2} 5^{-1}\right)$ & 2 & 96 & $\mathrm{I}\left(2_{1}^{+1} 16_{7}^{+1} 3^{-1}\right)$ \\
\hline 96 & $\mathrm{I}\left(2_{1}^{+1} 16_{1}^{+1} 3^{+1}\right)$ & 2 & 96 & $\mathrm{I}\left(2_{1}^{+1} 16_{7}^{+1} 3^{+1}\right)$ & 2 & 96 & $\mathrm{I}\left(2_{1}^{+1} 16_{1}^{+1} 3^{-1}\right)$ \\
\hline 96 & $\mathrm{I}\left(2_{1}^{+1} 16_{3}^{-1} 3^{+1}\right)$ & 2 & 96 & $\mathrm{I}\left(2_{1}^{+1} 16_{5}^{-1} 3^{-1}\right)$ & 1 & 96 & $\mathrm{I}\left(2_{1}^{+1} 16_{3}^{-1} 3^{-1}\right)$ \\
\hline 96 & $\mathrm{I}\left(2_{1}^{+1} 16_{5}^{-1} 3^{+1}\right)$ & 2 & 98 & $\mathrm{II}\left(2_{7}^{+1} 7^{+2}\right)$ & 1 & 98 & $\mathrm{I}\left(2_{1}^{+1} 7^{+2}\right)$ \\
\hline 99 & $\mathrm{I}\left(3^{+2} 11^{+1}\right)$ & 2 & 99 & $\mathrm{I}\left(3^{-2} 11^{-1}\right)$ & 2 & 100 & $\mathrm{I}\left(2_{2}^{+2} 5^{+2}\right)$ \\
\hline 100 & $\mathrm{I}\left(2_{2}^{+2} 5^{-2}\right)$ & 1 & 100 & $\mathrm{II}\left(4_{3}^{-1} 5^{+2}\right)$ & 1 & 100 & $\mathrm{II}\left(4_{7}^{+1} 5^{-2}\right)$ \\
\hline 100 & $\mathrm{I}\left(4_{1}^{+1} 5^{+2}\right)$ & 2 & 100 & $\mathrm{I}\left(4_{1}^{+1} 5^{-2}\right)$ & 1 & 105 & $\mathrm{I}\left(3^{+1} 5^{-1} 7^{+1}\right)$ \\
\hline 105 & $\mathrm{I}\left(3^{-1} 5^{-1} 7^{-1}\right)$ & 2 & 105 & $\mathrm{I}\left(3^{-1} 5^{+1} 7^{+1}\right)$ & 2 & 105 & $\mathrm{I}\left(3^{-1} 5^{-1} 7^{+1}\right)$ \\
\hline 108 & $\mathrm{I}\left(2_{2}^{+2} 3^{+1} 9^{-1}\right)$ & 1 & 108 & $\mathrm{I}\left(2_{2}^{+2} 3^{+1} 9^{+1}\right)$ & 1 & 108 & $\mathrm{I}\left(2_{\mathrm{II}}^{+2} 3^{+1} 9^{+1}\right)$ \\
\hline
\end{tabular}


B. PRIMITIVE TOTALLY-REFLECTIVE GENERA OF DIMENSION 3

67

\begin{tabular}{|c|c|c|c|c|c|c|c|}
\hline 108 & $\mathrm{I}\left(2_{2}^{+2} 3^{-1} 9^{+1}\right)$ & 1 & 108 & $\mathrm{I}\left(2_{2}^{+2} 3^{-1} 9^{-1}\right)$ & 1 & 108 & $\operatorname{II}\left(4_{1}^{+1} 3^{-1} 9^{+1}\right)$ \\
\hline 108 & $\mathrm{II}\left(4_{1}^{+1} 3^{-1} 9^{-1}\right)$ & 1 & 108 & $\mathrm{I}\left(4_{7}^{+1} 3^{-1} 9^{+1}\right)$ & 1 & 108 & $\mathrm{I}\left(4_{7}^{+1} 3^{-1} 9^{-1}\right)$ \\
\hline 108 & $\mathrm{I}\left(4_{1}^{+1} 3^{+1} 9^{+1}\right)$ & 2 & 108 & $\mathrm{I}\left(4_{7}^{+1} 3^{+1} 9^{-1}\right)$ & 1 & 108 & $\mathrm{I}\left(4_{7}^{+1} 3^{+1} 9^{+1}\right)$ \\
\hline 108 & $\operatorname{II}\left(4_{5}^{-1} 3^{+1} 9^{-1}\right)$ & 1 & 108 & $\operatorname{II}\left(4_{5}^{-1} 3^{+1} 9^{+1}\right)$ & 1 & 112 & $\mathrm{I}\left(4_{\mathrm{II}}^{+2} 7^{+1}\right)$ \\
\hline 112 & $\mathrm{I}\left(4_{2}^{+2} 7^{+1}\right)$ & 2 & 117 & $\mathrm{I}\left(3^{+2} 13^{-1}\right)$ & 1 & 120 & $\mathrm{I}\left(2_{1}^{+1} 4_{1}^{+1} 3^{+1} 5^{-1}\right)$ \\
\hline 120 & $\mathrm{I}\left(2_{1}^{+1} 4_{1}^{+1} 3^{+1} 5^{+1}\right)$ & 3 & 121 & $\mathrm{I}\left(11^{-2}\right)$ & 1 & 125 & $\mathrm{I}\left(5^{+1} 25^{-1}\right)$ \\
\hline 125 & $\mathrm{I}\left(5^{+1} 25^{+1}\right)$ & 1 & 126 & $\mathrm{II}\left(2_{1}^{+1} 3^{+2} 7^{-1}\right)$ & 2 & 126 & $\operatorname{II}\left(2_{1}^{+1} 9^{-1} 7^{+1}\right)$ \\
\hline 126 & $\mathrm{I}\left(2_{1}^{+1} 3^{+2} 7^{-1}\right)$ & 2 & 126 & $\mathrm{I}\left(2_{1}^{+1} 3^{+2} 7^{+1}\right)$ & 2 & 128 & $\mathrm{I}\left(2_{1}^{+1} 64_{1}^{+1}\right)$ \\
\hline 132 & $\mathrm{I}\left(2_{2}^{+2} 3^{-1} 11^{+1}\right)$ & 3 & 132 & $\mathrm{II}\left(4_{3}^{-1} 3^{+1} 11^{-1}\right)$ & 2 & 132 & $\operatorname{II}\left(4_{3}^{-1} 3^{-1} 11^{+1}\right)$ \\
\hline 132 & $\mathrm{I}\left(4_{1}^{+1} 3^{-1} 11^{+1}\right)$ & 3 & 135 & $\mathrm{I}\left(3^{+1} 9^{+1} 5^{+1}\right)$ & 2 & 135 & $\mathrm{I}\left(3^{-1} 9^{+1} 5^{-1}\right)$ \\
\hline 135 & $\mathrm{I}\left(3^{+1} 9^{-1} 5^{+1}\right)$ & 2 & 135 & $\mathrm{I}\left(3^{-1} 9^{-1} 5^{+1}\right)$ & 2 & 135 & $\mathrm{I}\left(3^{+1} 9^{+1} 5^{-1}\right)$ \\
\hline 135 & $\mathrm{I}\left(3^{+1} 9^{-1} 5^{-1}\right)$ & 1 & 140 & $\operatorname{II}\left(4_{1}^{+1} 5^{+1} 7^{-1}\right)$ & 1 & 140 & $\operatorname{II}\left(4_{1}^{+1} 5^{-1} 7^{+1}\right)$ \\
\hline 144 & $\mathrm{I}\left(4_{\mathrm{II}}^{-2} 3^{-2}\right)$ & 1 & 144 & $\mathrm{I}\left(4_{\mathrm{II}}^{-2} 9^{-1}\right)$ & 1 & 144 & $\mathrm{I}\left(4_{\mathrm{II}}^{-2} 9^{+1}\right)$ \\
\hline 144 & $\mathrm{I}\left(4_{2}^{+2} 3^{-2}\right)$ & 1 & 144 & $\mathrm{I}\left(4_{2}^{+2} 9^{+1}\right)$ & 2 & 144 & $\mathrm{I}\left(4_{6}^{+2} 3^{+2}\right)$ \\
\hline 144 & $\mathrm{I}\left(4_{\mathrm{II}}^{+2} 3^{+2}\right)$ & 1 & 144 & $\mathrm{I}\left(4_{0}^{+2} 3^{+2}\right)$ & 1 & 144 & $\mathrm{II}\left(16_{3}^{-1} 3^{-2}\right)$ \\
\hline 144 & $\mathrm{I}\left(16_{5}^{-1} 3^{+2}\right)$ & 1 & 144 & $\mathrm{I}\left(16_{3}^{-1} 3^{+2}\right)$ & 2 & 144 & $\mathrm{I}\left(16_{5}^{-1} 9^{+1}\right)$ \\
\hline 144 & $\mathrm{I}\left(16_{7}^{+1} 3^{+2}\right)$ & 2 & 144 & $\mathrm{I}\left(16_{1}^{+1} 9^{+1}\right)$ & 4 & 144 & $\mathrm{I}\left(16_{1}^{+1} 3^{+2}\right)$ \\
\hline 147 & $\mathrm{I}\left(3^{-1} 7^{-2}\right)$ & 2 & 147 & $\mathrm{I}\left(3^{+1} 7^{+2}\right)$ & 1 & 147 & $\mathrm{I}\left(3^{+1} 7^{-2}\right)$ \\
\hline 150 & $\operatorname{II}\left(2_{1}^{+1} 3^{+1} 5^{-2}\right)$ & 2 & 150 & $\operatorname{II}\left(2_{1}^{+1} 3^{-1} 5^{+2}\right)$ & 1 & 150 & $\mathrm{I}\left(2_{1}^{+1} 3^{+1} 5^{-2}\right)$ \\
\hline 150 & $\mathrm{I}\left(2_{1}^{+1} 3^{-1} 5^{+2}\right)$ & 2 & 150 & $\mathrm{I}\left(2_{1}^{+1} 3^{-1} 5^{-2}\right)$ & 1 & 153 & $\mathrm{I}\left(3^{+2} 17^{-1}\right)$ \\
\hline 156 & $\mathrm{I}\left(2_{\mathrm{II}}^{+2} 3^{+1} 13^{+1}\right)$ & 3 & 156 & $\mathrm{II}\left(4_{5}^{-1} 3^{+1} 13^{-1}\right)$ & 1 & 160 & $\mathrm{I}\left(2_{1}^{+1} 16_{1}^{+1} 5^{+1}\right)$ \\
\hline 160 & $\mathrm{I}\left(2_{1}^{+1} 16_{7}^{+1} 5^{+1}\right)$ & 2 & 160 & $\mathrm{I}\left(2_{1}^{+1} 16_{5}^{-1} 5^{+1}\right)$ & 2 & 160 & $\mathrm{I}\left(2_{1}^{+1} 16_{3}^{-1} 5^{+1}\right)$ \\
\hline 162 & $\mathrm{I}\left(2_{1}^{+1} 9^{+2}\right)$ & 2 & 165 & $\mathrm{I}\left(3^{+1} 5^{+1} 11^{-1}\right)$ & 2 & 171 & $\mathrm{I}\left(3^{+2} 19^{+1}\right)$ \\
\hline 175 & $\mathrm{I}\left(5^{+2} 7^{-1}\right)$ & 2 & 175 & $\mathrm{I}\left(5^{-2} 7^{+1}\right)$ & 1 & 176 & $\mathrm{I}\left(4_{\mathrm{II}}^{-2} 11^{-1}\right)$ \\
\hline 180 & $\mathrm{I}\left(2_{2}^{+2} 3^{+2} 5^{+1}\right)$ & 2 & 180 & $\mathrm{I}\left(2_{2}^{+2} 3^{-2} 5^{-1}\right)$ & 2 & 180 & $\mathrm{I}\left(2_{\mathrm{II}}^{+2} 3^{+2} 5^{+1}\right)$ \\
\hline 180 & $\mathrm{I}\left(2_{\mathrm{II}}^{+2} 3^{-2} 5^{-1}\right)$ & 2 & 180 & $\mathrm{I}\left(2_{2}^{+2} 3^{+2} 5^{-1}\right)$ & 1 & 180 & $\operatorname{II}\left(4_{7}^{+1} 3^{+2} 5^{-1}\right)$ \\
\hline 180 & $\operatorname{II}\left(4_{7}^{+1} 3^{-2} 5^{+1}\right)$ & 1 & 180 & $\operatorname{II}\left(4_{7}^{+1} 9^{-1} 5^{+1}\right)$ & 2 & 180 & $\mathrm{I}\left(4_{1}^{+1} 3^{+2} 5^{+1}\right)$ \\
\hline 180 & $\mathrm{I}\left(4_{1}^{+1} 3^{-2} 5^{-1}\right)$ & 2 & 180 & $\mathrm{I}\left(4_{1}^{+1} 3^{+2} 5^{-1}\right)$ & 1 & 180 & $\mathrm{II}\left(4_{3}^{-1} 3^{+2} 5^{+1}\right)$ \\
\hline 180 & $\mathrm{II}\left(4_{3}^{-1} 3^{-2} 5^{-1}\right)$ & 2 & 189 & $\mathrm{I}\left(3^{-1} 9^{+1} 7^{+1}\right)$ & 2 & 189 & $\mathrm{I}\left(3^{+1} 9^{-1} 7^{-1}\right)$ \\
\hline 189 & $\mathrm{I}\left(3^{-1} 9^{-1} 7^{+1}\right)$ & 2 & 189 & $\mathrm{I}\left(3^{+1} 9^{+1} 7^{-1}\right)$ & 1 & 192 & $\mathrm{I}\left(4_{1}^{+1} 16_{1}^{+1} 3^{+1}\right)$ \\
\hline 192 & $\mathrm{I}\left(4_{1}^{+1} 16_{7}^{+1} 3^{+1}\right)$ & 2 & 192 & $\mathrm{I}\left(4_{7}^{+1} 16_{1}^{+1} 3^{+1}\right)$ & 2 & 195 & $\mathrm{I}\left(3^{-1} 5^{-1} 13^{-1}\right)$ \\
\hline 196 & $\mathrm{I}\left(2_{2}^{+2} 7^{+2}\right)$ & 2 & 196 & $\mathrm{I}\left(2_{\mathrm{II}}^{+2} 7^{+2}\right)$ & 1 & 196 & $\mathrm{II}\left(4_{7}^{+1} 7^{+2}\right)$ \\
\hline 196 & $\mathrm{I}\left(4_{1}^{+1} 7^{+2}\right)$ & 2 & 198 & $\mathrm{I}\left(2_{1}^{+1} 3^{+2} 11^{-1}\right)$ & 3 & 200 & $\mathrm{I}\left(2_{1}^{+1} 4_{1}^{+1} 5^{-2}\right)$ \\
\hline 204 & $\mathrm{II}\left(4_{1}^{+1} 3^{-1} 17^{+1}\right)$ & 2 & 207 & $\mathrm{I}\left(3^{+2} 23^{+1}\right)$ & 3 & 210 & $\operatorname{III}\left(2_{7}^{+1} 3^{+1} 5^{+1} 7^{+1}\right)$ \\
\hline 210 & $\mathrm{I}\left(2_{1}^{+1} 3^{+1} 5^{+1} 7^{+1}\right)$ & 3 & 216 & $\mathrm{I}\left(2_{1}^{+1} 4_{1}^{+1} 3^{+1} 9^{+1}\right)$ & 2 & 216 & $\mathrm{I}\left(2_{1}^{+1} 4_{1}^{+1} 3^{+1} 9^{-1}\right)$ \\
\hline 216 & $\mathrm{I}\left(2_{1}^{+1} 4_{1}^{+1} 3^{-1} 9^{+1}\right)$ & 2 & 224 & $\mathrm{I}\left(2_{1}^{+1} 16_{5}^{-1} 7^{+1}\right)$ & 3 & 224 & $\mathrm{I}\left(2_{1}^{+1} 16_{3}^{-1} 7^{+1}\right)$ \\
\hline 224 & $\mathrm{I}\left(2_{1}^{+1} 16_{7}^{+1} 7^{+1}\right)$ & 3 & 224 & $\mathrm{I}\left(2_{1}^{+1} 16_{1}^{+1} 7^{+1}\right)$ & 3 & 225 & $\mathrm{I}\left(3^{-2} 5^{+2}\right)$ \\
\hline 225 & $\mathrm{I}\left(3^{+2} 5^{-2}\right)$ & 1 & 225 & $\mathrm{I}\left(9^{+1} 5^{+2}\right)$ & 2 & 225 & $\mathrm{I}\left(3^{-2} 5^{-2}\right)$ \\
\hline 225 & $\mathrm{I}\left(3^{+2} 5^{+2}\right)$ & 2 & 228 & $\mathrm{II}\left(4_{3}^{-1} 3^{+1} 19^{-1}\right)$ & 2 & 234 & $\operatorname{II}\left(2_{7}^{+1} 3^{+2} 13^{-1}\right)$ \\
\hline
\end{tabular}




\begin{tabular}{|c|c|c|c|c|c|c|c|}
\hline 240 & $\mathrm{I}\left(4_{\mathrm{II}}^{-2} 3^{+1} 5^{-1}\right)$ & 1 & 240 & $\mathrm{I}\left(4_{\mathrm{II}}^{-2} 3^{-1} 5^{+1}\right)$ & 1 & 240 & $\mathrm{I}\left(4_{\mathrm{II}}^{+2} 3^{+1} 5^{+1}\right)$ \\
\hline 240 & $\mathrm{I}\left(4_{\mathrm{II}}^{+2} 3^{-1} 5^{-1}\right)$ & 2 & 240 & $\mathrm{I}\left(4_{2}^{+2} 3^{+1} 5^{+1}\right)$ & 2 & 240 & $\mathrm{I}\left(4_{2}^{+2} 3^{-1} 5^{-1}\right)$ \\
\hline 240 & $\mathrm{I}\left(4_{6}^{+2} 3^{-1} 5^{+1}\right)$ & 1 & 240 & $\operatorname{II}\left(16_{5}^{-1} 3^{-1} 5^{+1}\right)$ & 2 & 245 & $\mathrm{I}\left(5^{-1} 7^{+2}\right)$ \\
\hline 245 & $\mathrm{I}\left(5^{+1} 7^{-2}\right)$ & 2 & 250 & $\mathrm{I}\left(2_{1}^{+1} 5^{-1} 25^{-1}\right)$ & 3 & 252 & $\mathrm{I}\left(2_{\mathrm{II}}^{+2} 3^{+2} 7^{-1}\right)$ \\
\hline 252 & $\mathrm{I}\left(2_{\mathrm{II}}^{+2} 9^{+1} 7^{+1}\right)$ & 3 & 252 & $\mathrm{I}\left(2_{2}^{+2} 3^{+2} 7^{+1}\right)$ & 2 & 252 & $\mathrm{I}\left(2_{2}^{+2} 3^{+2} 7^{-1}\right)$ \\
\hline 52 & $\operatorname{II}\left(4_{5}^{-1} 3^{-2} 7^{-1}\right)$ & 2 & 252 & $\operatorname{II}\left(4_{5}^{-1} 3^{+2} 7^{+1}\right)$ & 1 & 252 & $\operatorname{II}\left(4_{1}^{+1} 3^{+2} 7^{-1}\right)$ \\
\hline 52 & $\mathrm{I}\left(4_{7}^{+1} 3^{+2} 7^{-1}\right)$ & 2 & 252 & $\mathrm{I}\left(4_{7}^{+1} 3^{+2} 7^{+1}\right)$ & 2 & 256 & $\mathrm{I}\left(16_{6}^{-2}\right)$ \\
\hline 256 & $\mathrm{I}\left(16_{2}^{+2}\right)$ & 2 & 260 & $\operatorname{II}\left(4_{3}^{-1} 5^{+1} 13^{+1}\right)$ & 2 & 270 & $\mathrm{II}\left(2_{1}^{+1} 3^{-1} 9^{+1} 5^{-1}\right)$ \\
\hline 270 & $\mathrm{II}\left(2_{1}^{+1} 3^{+1} 9^{-1} 5^{+1}\right)$ & 2 & 270 & $\mathrm{II}\left(2_{1}^{+1} 3^{-1} 9^{-1} 5^{-1}\right)$ & 2 & 270 & $\mathrm{I}\left(2_{1}^{+1} 3^{-1} 9^{+1} 5^{-1}\right)$ \\
\hline 270 & $\mathrm{I}\left(2_{1}^{+1} 3^{+1} 9^{-1} 5^{+1}\right)$ & 3 & 270 & $\mathrm{I}\left(2_{1}^{+1} 3^{-1} 9^{-1} 5^{-1}\right)$ & 2 & 270 & $\mathrm{I}\left(2_{1}^{+1} 3^{+1} 9^{+1} 5^{-1}\right)$ \\
\hline 272 & $\mathrm{I}\left(4_{\mathrm{II}}^{-2} 17^{+1}\right)$ & 2 & 276 & $\mathrm{II}\left(4_{7}^{+1} 3^{-1} 23^{+1}\right)$ & 3 & 288 & $\mathrm{I}\left(2_{1}^{+1} 16_{5}^{-1} 3^{+2}\right)$ \\
\hline 288 & $\mathrm{I}\left(2_{1}^{+1} 16_{3}^{-1} 3^{+2}\right)$ & 1 & 288 & $\mathrm{I}\left(2_{1}^{+1} 16_{5}^{-1} 3^{-2}\right)$ & 2 & 288 & $\mathrm{I}\left(2_{1}^{+1} 16_{3}^{-1} 3^{-2}\right)$ \\
\hline 288 & $\mathrm{I}\left(2_{1}^{+1} 16_{1}^{+1} 3^{-2}\right)$ & 2 & 288 & $\mathrm{I}\left(2_{1}^{+1} 16_{7}^{+1} 3^{-2}\right)$ & 2 & 288 & $\mathrm{I}\left(2_{1}^{+1} 16_{1}^{+1} 3^{+2}\right)$ \\
\hline 288 & $\mathrm{I}\left(2_{1}^{+1} 16_{7}^{+1} 3^{+2}\right)$ & 1 & 289 & $\mathrm{I}\left(17^{+2}\right)$ & 2 & 294 & $\mathrm{II}\left(2_{1}^{+1} 3^{-1} 7^{-2}\right)$ \\
\hline 294 & $\mathrm{I}\left(2_{1}^{+1} 3^{-1} 7^{-2}\right)$ & 2 & 294 & $\mathrm{I}\left(2_{1}^{+1} 3^{-1} 7^{+2}\right)$ & 2 & 297 & $\mathrm{I}\left(3^{-1} 9^{-1} 11^{+1}\right)$ \\
\hline 297 & $\mathrm{I}\left(3^{+1} 9^{+1} 11^{-1}\right)$ & 2 & 297 & $\mathrm{I}\left(3^{+1} 9^{-1} 11^{-1}\right)$ & 2 & 300 & $\mathrm{I}\left(2_{2}^{+2} 3^{-1} 5^{-2}\right)$ \\
\hline 300 & $\mathrm{I}\left(2_{2}^{+2} 3^{+1} 5^{+2}\right)$ & 2 & 300 & $\mathrm{I}\left(2_{\mathrm{II}}^{+2} 3^{-1} 5^{-2}\right)$ & 2 & 300 & $\mathrm{I}\left(2_{\mathrm{II}}^{+2} 3^{+1} 5^{+2}\right)$ \\
\hline 300 & $\mathrm{I}\left(2_{2}^{+2} 3^{+1} 5^{-2}\right)$ & 1 & 300 & $\mathrm{II}\left(4_{1}^{+1} 3^{-1} 5^{+2}\right)$ & 1 & 300 & $\operatorname{II}\left(4_{1}^{+1} 3^{+1} 5^{-2}\right)$ \\
\hline 300 & $\mathrm{I}\left(4_{7}^{+1} 3^{+1} 5^{-2}\right)$ & 1 & 300 & $\mathrm{I}\left(4_{7}^{+1} 3^{-1} 5^{-2}\right)$ & 2 & 300 & $\mathrm{I}\left(4_{7}^{+1} 3^{+1} 5^{+2}\right)$ \\
\hline 300 & $\operatorname{II}\left(4_{5}^{-1} 3^{-1} 5^{-2}\right)$ & 2 & 300 & $\mathrm{II}\left(4_{5}^{-1} 3^{+1} 5^{+2}\right)$ & 2 & 308 & $\mathrm{II}\left(4_{7}^{+1} 7^{+1} 11^{-1}\right)$ \\
\hline 315 & $\mathrm{I}\left(3^{+2} 5^{+1} 7^{+1}\right)$ & 2 & 315 & $\mathrm{I}\left(3^{+2} 5^{-1} 7^{-1}\right)$ & 2 & 315 & $\mathrm{I}\left(3^{-2} 5^{+1} 7^{-1}\right)$ \\
\hline 315 & $\mathrm{I}\left(3^{+2} 5^{+1} 7^{-1}\right)$ & 3 & 320 & $\mathrm{I}\left(4_{1}^{+1} 16_{1}^{+1} 5^{+1}\right)$ & 6 & 324 & $\mathrm{I}\left(2_{2}^{+2} 9^{+2}\right)$ \\
\hline 324 & $\operatorname{II}\left(4_{3}^{-1} 3^{+1} 27^{-1}\right)$ & 1 & 324 & $\operatorname{II}\left(4_{3}^{-1} 9^{-2}\right)$ & 1 & 324 & $\mathrm{II}\left(4_{3}^{-1} 9^{+2}\right)$ \\
\hline 324 & $\mathrm{I}\left(4_{1}^{+1} 9^{+2}\right)$ & 2 & 325 & $\mathrm{I}\left(5^{-2} 13^{-1}\right)$ & 2 & 330 & $\mathrm{II}\left(2_{7}^{+1} 3^{-1} 5^{-1} 11^{-1}\right)$ \\
\hline 336 & $\mathrm{I}\left(4_{\mathrm{II}}^{+2} 3^{+1} 7^{+1}\right)$ & 2 & 336 & $\mathrm{I}\left(4_{2}^{+2} 3^{+1} 7^{-1}\right)$ & 2 & 336 & $\mathrm{I}\left(4_{6}^{+2} 3^{+1} 7^{+1}\right)$ \\
\hline 336 & $\mathrm{I}\left(4_{\mathrm{II}}^{-2} 3^{-1} 7^{+1}\right)$ & 2 & 336 & $\mathrm{I}\left(4_{\mathrm{II}}^{-2} 3^{+1} 7^{-1}\right)$ & 1 & 336 & $\mathrm{I}\left(16_{1}^{+1} 3^{+1} 7^{-1}\right)$ \\
\hline 336 & $\mathrm{I}\left(16_{5}^{-1} 3^{+1} 7^{-1}\right)$ & 4 & 351 & $\mathrm{I}\left(3^{+1} 9^{-1} 13^{-1}\right)$ & 2 & 360 & $\mathrm{I}\left(2_{1}^{+1} 4_{1}^{+1} 3^{+2} 5^{+1}\right)$ \\
\hline 360 & $\mathrm{I}\left(2_{1}^{+1} 4_{1}^{+1} 3^{+2} 5^{-1}\right)$ & 3 & 363 & $\mathrm{I}\left(3^{-1} 11^{-2}\right)$ & 2 & 363 & $\mathrm{I}\left(3^{+1} 11^{+2}\right)$ \\
\hline 375 & $\mathrm{I}\left(3^{-1} 5^{-1} 25^{+1}\right)$ & 3 & 375 & $\mathrm{I}\left(3^{+1} 5^{-1} 25^{-1}\right)$ & 2 & 375 & $\mathrm{I}\left(3^{-1} 5^{+1} 25^{+1}\right)$ \\
\hline 375 & $\mathrm{I}\left(3^{-1} 5^{+1} 25^{-1}\right)$ & 1 & 378 & $\operatorname{II}\left(2_{7}^{+1} 3^{-1} 9^{-1} 7^{+1}\right)$ & 4 & 378 & $\mathrm{I}\left(2_{1}^{+1} 3^{-1} 9^{-1} 7^{-1}\right)$ \\
\hline 384 & $\mathrm{I}\left(2_{1}^{+1} 64_{1}^{+1} 3^{-1}\right)$ & 4 & 392 & $\mathrm{I}\left(2_{1}^{+1} 4_{1}^{+1} 7^{+2}\right)$ & 3 & 396 & $\mathrm{I}\left(2_{2}^{+2} 3^{+2} 11^{+1}\right)$ \\
\hline 396 & $\mathrm{II}\left(4_{1}^{+1} 3^{+2} 11^{+1}\right)$ & 2 & 396 & $\mathrm{II}\left(4_{1}^{+1} 3^{-2} 11^{-1}\right)$ & 2 & 396 & $\mathrm{I}\left(4_{7}^{+1} 3^{+2} 11^{+1}\right)$ \\
\hline 400 & $\mathrm{I}\left(4_{\mathrm{II}}^{-2} 5^{+2}\right)$ & 1 & 400 & $\mathrm{I}\left(4_{2}^{+2} 5^{+2}\right)$ & 2 & 400 & $\mathrm{I}\left(4_{6}^{+2} 5^{-2}\right)$ \\
\hline 400 & $\mathrm{I}\left(4_{\mathrm{II}}^{+2} 5^{-2}\right)$ & 1 & 400 & $\mathrm{I}\left(16_{5}^{-1} 5^{-2}\right)$ & 2 & 400 & $\mathrm{I}\left(16_{5}^{-1} 5^{+2}\right)$ \\
\hline 400 & $\mathrm{I}\left(16_{1}^{+1} 5^{+2}\right)$ & 4 & 405 & $\mathrm{I}\left(9^{+2} 5^{+1}\right)$ & 2 & 420 & $\mathrm{I}\left(2_{2}^{+2} 3^{-1} 5^{-1} 7^{+1}\right)$ \\
\hline 420 & $\mathrm{I}\left(2_{\mathrm{II}}^{+2} 3^{-1} 5^{-1} 7^{+1}\right)$ & 2 & 420 & $\mathrm{II}\left(4_{3}^{-1} 3^{+1} 5^{-1} 7^{+1}\right)$ & 2 & 420 & $\mathrm{II}\left(4_{3}^{-1} 3^{-1} 5^{-1} 7^{-1}\right)$ \\
\hline 420 & $\mathrm{II}\left(4_{3}^{-1} 3^{-1} 5^{+1} 7^{+1}\right)$ & 2 & 420 & $\mathrm{II}\left(4_{7}^{+1} 3^{-1} 5^{-1} 7^{+1}\right)$ & 3 & 420 & $\mathrm{I}\left(4_{1}^{+1} 3^{-1} 5^{-1} 7^{+1}\right)$ \\
\hline 432 & $\mathrm{I}\left(4_{2}^{+2} 3^{+1} 9^{-1}\right)$ & 1 & 432 & $\mathrm{I}\left(4_{2}^{+2} 3^{+1} 9^{+1}\right)$ & 1 & 432 & $\mathrm{I}\left(4_{6}^{+2} 3^{-1} 9^{+1}\right)$ \\
\hline 432 & $\mathrm{I}\left(4_{6}^{+2} 3^{-1} 9^{-1}\right)$ & 1 & 432 & $\mathrm{I}\left(4_{\mathrm{II}}^{+2} 3^{+1} 9^{-1}\right)$ & 1 & 432 & $\mathrm{I}\left(4_{\mathrm{II}}^{+2} 3^{+1} 9^{+1}\right)$ \\
\hline
\end{tabular}


B. PRIMITIVE TOTALLY-REFLECTIVE GENERA OF DIMENSION 3

69

\begin{tabular}{|c|c|c|c|c|c|c|c|}
\hline 432 & $\mathrm{I}\left(4_{0}^{+2} 3^{+1} 9^{+1}\right)$ & 2 & 432 & $\mathrm{I}\left(4_{\mathrm{II}}^{-2} 3^{-1} 9^{+1}\right)$ & 1 & 432 & $\mathrm{I}\left(4_{\mathrm{II}}^{-2} 3^{-1} 9^{-1}\right)$ \\
\hline 432 & $\mathrm{II}\left(16_{1}^{+1} 3^{-1} 9^{+1}\right)$ & 2 & 432 & $\mathrm{II}\left(16_{1}^{+1} 3^{-1} 9^{-1}\right)$ & 2 & 432 & $\mathrm{I}\left(16_{1}^{+1} 3^{+1} 9^{+1}\right)$ \\
\hline 432 & $\mathrm{I}\left(16_{7}^{+1} 3^{+1} 9^{+1}\right)$ & 2 & 432 & $\mathrm{I}\left(16_{5}^{-1} 3^{+1} 9^{+1}\right)$ & 4 & 432 & $\mathrm{I}\left(16_{3}^{-1} 3^{+1} 9^{-1}\right)$ \\
\hline 432 & $\mathrm{I}\left(16_{3}^{-1} 3^{+1} 9^{+1}\right)$ & 2 & 441 & $\mathrm{I}\left(3^{-2} 7^{-2}\right)$ & 2 & 441 & $\mathrm{I}\left(3^{+2} 7^{+2}\right)$ \\
\hline 441 & $\mathrm{I}\left(3^{+2} 7^{-2}\right)$ & 2 & 450 & $\mathrm{II}\left(2_{7}^{+1} 3^{+2} 5^{+2}\right)$ & 1 & 450 & $\mathrm{II}\left(2_{7}^{+1} 3^{-2} 5^{-2}\right)$ \\
\hline 450 & $\mathrm{I}\left(2_{1}^{+1} 3^{+2} 5^{-2}\right)$ & 1 & 450 & $\mathrm{I}\left(2_{1}^{+1} 3^{+2} 5^{+2}\right)$ & 2 & 450 & $\mathrm{I}\left(2_{1}^{+1} 3^{-2} 5^{-2}\right)$ \\
\hline 468 & $\mathrm{I}\left(2_{\mathrm{II}}^{+2} 3^{+2} 13^{+1}\right)$ & 3 & 468 & $\operatorname{II}\left(4_{7}^{+1} 3^{+2} 13^{-1}\right)$ & 1 & 480 & $\mathrm{I}\left(2_{1}^{+1} 16_{3}^{-1} 3^{+1} 5^{-1}\right)$ \\
\hline 480 & $\mathrm{I}\left(2_{1}^{+1} 16_{5}^{-1} 3^{+1} 5^{+1}\right)$ & 3 & 480 & $\mathrm{I}\left(2_{1}^{+1} 16_{3}^{-1} 3^{+1} 5^{+1}\right)$ & 3 & 480 & $\mathrm{I}\left(2_{1}^{+1} 16_{5}^{-1} 3^{+1} 5^{-1}\right)$ \\
\hline 480 & $\mathrm{I}\left(2_{1}^{+1} 16_{7}^{+1} 3^{+1} 5^{+1}\right)$ & 3 & 480 & $\mathrm{I}\left(2_{1}^{+1} 16_{1}^{+1} 3^{+1} 5^{-1}\right)$ & 2 & 480 & $\mathrm{I}\left(2_{1}^{+1} 16_{7}^{+1} 3^{+1} 5^{-1}\right)$ \\
\hline 480 & $\mathrm{I}\left(2_{1}^{+1} 16_{1}^{+1} 3^{+1} 5^{+1}\right)$ & 3 & 484 & $\mathrm{II}\left(4_{3}^{-1} 11^{-2}\right)$ & 1 & 495 & $\mathrm{I}\left(3^{+2} 5^{-1} 11^{-1}\right)$ \\
\hline 500 & $\mathrm{I}\left(2_{2}^{+2} 5^{+1} 25^{+1}\right)$ & 3 & 500 & $\mathrm{II}\left(4_{7}^{+1} 5^{+1} 25^{-1}\right)$ & 1 & 500 & $\mathrm{II}\left(4_{7}^{+1} 5^{+1} 25^{+1}\right)$ \\
\hline 500 & $\mathrm{I}\left(4_{1}^{+1} 5^{+1} 25^{+1}\right)$ & 3 & 507 & $\mathrm{I}\left(3^{+1} 13^{-2}\right)$ & 1 & 513 & $\mathrm{I}\left(3^{+1} 9^{-1} 19^{-1}\right)$ \\
\hline 525 & $\mathrm{I}\left(3^{+1} 5^{-2} 7^{-1}\right)$ & 3 & 525 & $\mathrm{I}\left(3^{-1} 5^{-2} 7^{-1}\right)$ & 2 & 525 & $\mathrm{I}\left(3^{+1} 5^{-2} 7^{+1}\right)$ \\
\hline 525 & $\mathrm{I}\left(3^{+1} 5^{+2} 7^{-1}\right)$ & 2 & 528 & $\mathrm{I}\left(4_{\mathrm{II}}^{-2} 3^{+1} 11^{-1}\right)$ & 2 & 528 & $\mathrm{I}\left(4_{\mathrm{II}}^{-2} 3^{-1} 11^{+1}\right)$ \\
\hline 528 & $\mathrm{I}\left(4_{2}^{+2} 3^{-1} 11^{+1}\right)$ & 3 & 539 & $\mathrm{I}\left(7^{+2} 11^{+1}\right)$ & 3 & 540 & $\mathrm{I}\left(2_{\mathrm{II}}^{+2} 3^{+1} 9^{+1} 5^{+1}\right)$ \\
\hline 540 & $\mathrm{I}\left(2_{\mathrm{II}}^{+2} 3^{-1} 9^{+1} 5^{-1}\right)$ & 2 & 540 & $\mathrm{I}\left(2_{\mathrm{II}}^{+2} 3^{+1} 9^{-1} 5^{+1}\right)$ & 2 & 540 & $\mathrm{I}\left(2_{2}^{+2} 3^{-1} 9^{-1} 5^{+1}\right)$ \\
\hline 540 & $\mathrm{I}\left(2_{2}^{+2} 3^{+1} 9^{+1} 5^{+1}\right)$ & 2 & 540 & $\mathrm{I}\left(2_{2}^{+2} 3^{-1} 9^{+1} 5^{-1}\right)$ & 3 & 540 & $\mathrm{I}\left(2_{2}^{+2} 3^{+1} 9^{-1} 5^{+1}\right)$ \\
\hline 540 & $\operatorname{II}\left(4_{5}^{-1} 3^{-1} 9^{-1} 5^{+1}\right)$ & 2 & 540 & $\mathrm{II}\left(4_{5}^{-1} 3^{+1} 9^{+1} 5^{-1}\right)$ & 1 & 540 & $\operatorname{II}\left(4_{5}^{-1} 3^{+1} 9^{-1} 5^{-1}\right)$ \\
\hline 540 & $\operatorname{II}\left(4_{1}^{+1} 3^{+1} 9^{+1} 5^{+1}\right)$ & 2 & 540 & $\operatorname{II}\left(4_{1}^{+1} 3^{-1} 9^{+1} 5^{-1}\right)$ & 3 & 540 & $\operatorname{II}\left(4_{1}^{+1} 3^{+1} 9^{-1} 5^{+1}\right)$ \\
\hline 540 & $\mathrm{I}\left(4_{7}^{+1} 3^{+1} 9^{+1} 5^{+1}\right)$ & 2 & 540 & $\mathrm{I}\left(4_{7}^{+1} 3^{-1} 9^{+1} 5^{-1}\right)$ & 3 & 540 & $\mathrm{I}\left(4_{7}^{+1} 3^{+1} 9^{-1} 5^{+1}\right)$ \\
\hline 540 & $\mathrm{I}\left(4_{7}^{+1} 3^{-1} 9^{-1} 5^{+1}\right)$ & 2 & 560 & $\mathrm{I}\left(4_{\mathrm{II}}^{-2} 5^{+1} 7^{-1}\right)$ & 1 & 560 & $\mathrm{I}\left(4_{\mathrm{II}}^{-2} 5^{-1} 7^{+1}\right)$ \\
\hline 576 & $\mathrm{I}\left(4_{1}^{+1} 16_{1}^{+1} 9^{+1}\right)$ & 6 & 576 & $\mathrm{I}\left(4_{7}^{+1} 16_{7}^{+1} 3^{+2}\right)$ & 2 & 576 & $\mathrm{I}\left(4_{1}^{+1} 16_{7}^{+1} 3^{+2}\right)$ \\
\hline 576 & $\mathrm{I}\left(4_{7}^{+1} 16_{1}^{+1} 3^{+2}\right)$ & 2 & 585 & $\mathrm{I}\left(3^{+2} 5^{+1} 13^{-1}\right)$ & 3 & 588 & $\mathrm{I}\left(2_{2}^{+2} 3^{+1} 7^{-2}\right)$ \\
\hline 588 & $\mathrm{I}\left(2_{\mathrm{II}}^{+2} 3^{+1} 7^{-2}\right)$ & 2 & 588 & $\mathrm{I}\left(2_{2}^{+2} 3^{+1} 7^{+2}\right)$ & 2 & 588 & $\mathrm{II}\left(4_{1}^{+1} 3^{-1} 7^{-2}\right)$ \\
\hline 588 & $\mathrm{II}\left(4_{1}^{+1} 3^{+1} 7^{+2}\right)$ & 1 & 588 & $\mathrm{I}\left(4_{7}^{+1} 3^{+1} 7^{+2}\right)$ & 2 & 588 & $\mathrm{I}\left(4_{7}^{+1} 3^{+1} 7^{-2}\right)$ \\
\hline 588 & $\mathrm{II}\left(4_{5}^{-1} 3^{+1} 7^{-2}\right)$ & 2 & 600 & $\mathrm{I}\left(2_{1}^{+1} 4_{1}^{+1} 3^{-1} 5^{-2}\right)$ & 2 & 600 & $\mathrm{I}\left(2_{1}^{+1} 4_{1}^{+1} 3^{-1} 5^{+2}\right)$ \\
\hline 612 & $\operatorname{II}\left(4_{3}^{-1} 3^{+2} 17^{-1}\right)$ & 2 & 624 & $\mathrm{I}\left(4_{\mathrm{II}}^{-2} 3^{+1} 13^{-1}\right)$ & 1 & 630 & $\operatorname{II}\left(2_{1}^{+1} 3^{+2} 5^{-1} 7^{-1}\right)$ \\
\hline 630 & $\mathrm{I}\left(2_{1}^{+1} 3^{+2} 5^{-1} 7^{-1}\right)$ & 3 & 660 & $\mathrm{I}\left(2_{\mathrm{II}}^{+2} 3^{+1} 5^{+1} 11^{+1}\right)$ & 4 & 660 & $\mathrm{II}\left(4_{7}^{+1} 3^{+1} 5^{+1} 11^{-1}\right)$ \\
\hline 675 & $\mathrm{I}\left(3^{-1} 9^{+1} 5^{+2}\right)$ & 1 & 675 & $\mathrm{I}\left(3^{-1} 9^{-1} 5^{+2}\right)$ & 1 & 675 & $\mathrm{I}\left(3^{+1} 9^{+1} 5^{-2}\right)$ \\
\hline 675 & $\mathrm{I}\left(3^{-1} 9^{+1} 5^{-2}\right)$ & 2 & 675 & $\mathrm{I}\left(3^{+1} 9^{-1} 5^{+2}\right)$ & 3 & 675 & $\mathrm{I}\left(3^{-1} 9^{-1} 5^{-2}\right)$ \\
\hline 684 & $\operatorname{II}\left(4_{1}^{+1} 3^{+2} 19^{+1}\right)$ & 2 & 700 & $\mathrm{II}\left(4_{5}^{-1} 5^{+2} 7^{-1}\right)$ & 2 & 700 & $\mathrm{II}\left(4_{5}^{-1} 5^{-2} 7^{+1}\right)$ \\
\hline 702 & $\operatorname{II}\left(2_{1}^{+1} 3^{-1} 9^{-1} 13^{-1}\right)$ & 6 & 720 & $\mathrm{I}\left(4_{\mathrm{II}}^{+2} 3^{+2} 5^{+1}\right)$ & 2 & 720 & $\mathrm{I}\left(4_{\mathrm{II}}^{+2} 3^{-2} 5^{-1}\right)$ \\
\hline 720 & $\mathrm{I}\left(4_{2}^{+2} 3^{+2} 5^{-1}\right)$ & 1 & 720 & $\mathrm{I}\left(4_{6}^{+2} 3^{+2} 5^{+1}\right)$ & 2 & 720 & $\mathrm{I}\left(4_{6}^{+2} 3^{-2} 5^{-1}\right)$ \\
\hline 720 & $\mathrm{I}\left(4_{\mathrm{II}}^{-2} 3^{+2} 5^{-1}\right)$ & 1 & 720 & $\mathrm{I}\left(4_{\mathrm{II}}^{-2} 3^{-2} 5^{+1}\right)$ & 1 & 720 & $\mathrm{I}\left(4_{\mathrm{II}}^{-2} 9^{-1} 5^{+1}\right)$ \\
\hline 720 & $\mathrm{II}\left(16_{7}^{+1} 3^{+2} 5^{-1}\right)$ & 2 & 726 & $\mathrm{I}\left(2_{1}^{+1} 3^{-1} 11^{+2}\right)$ & 3 & 735 & $\mathrm{I}\left(3^{-1} 5^{+1} 7^{+2}\right)$ \\
\hline 735 & $\mathrm{I}\left(3^{+1} 5^{+1} 7^{+2}\right)$ & 2 & 735 & $\mathrm{I}\left(3^{-1} 5^{+1} 7^{-2}\right)$ & 2 & 735 & $\mathrm{I}\left(3^{-1} 5^{-1} 7^{+2}\right)$ \\
\hline 750 & $\mathrm{II}\left(2_{1}^{+1} 3^{+1} 5^{+1} 25^{-1}\right)$ & 2 & 750 & $\mathrm{I}\left(2_{1}^{+1} 3^{+1} 5^{+1} 25^{-1}\right)$ & 3 & 756 & $\mathrm{I}\left(2_{\mathrm{II}}^{+2} 3^{+1} 9^{+1} 7^{+1}\right)$ \\
\hline 756 & $\mathrm{I}\left(2_{2}^{+2} 3^{+1} 9^{+1} 7^{-1}\right)$ & 3 & 756 & $\mathrm{II}\left(4_{7}^{+1} 3^{-1} 9^{+1} 7^{+1}\right)$ & 2 & 756 & $\mathrm{II}\left(4_{7}^{+1} 3^{+1} 9^{-1} 7^{-1}\right)$ \\
\hline 756 & $\mathrm{II}\left(4_{7}^{+1} 3^{-1} 9^{-1} 7^{+1}\right)$ & 2 & 756 & $\mathrm{II}\left(4_{7}^{+1} 3^{+1} 9^{+1} 7^{-1}\right)$ & 1 & 756 & $\mathrm{I}\left(4_{1}^{+1} 3^{+1} 9^{+1} 7^{-1}\right)$ \\
\hline
\end{tabular}




\begin{tabular}{|c|c|c|c|c|c|c|c|}
\hline 768 & $\mathrm{I}\left(16_{2}^{+2} 3^{+1}\right)$ & 2 & 768 & $\mathrm{I}\left(16_{0}^{+2} 3^{+1}\right)$ & 2 & 768 & $\mathrm{I}\left(16_{\mathrm{II}}^{-2} 3^{-1}\right)$ \\
\hline 768 & $\mathrm{I}\left(16_{4}^{-2} 3^{+1}\right)$ & 2 & 768 & $\mathrm{I}\left(16_{6}^{-2} 3^{+1}\right)$ & 1 & 780 & $\mathrm{II}\left(4_{1}^{+1} 3^{-1} 5^{-1} 13^{-1}\right)$ \\
\hline 784 & $\mathrm{I}\left(4_{6}^{+2} 7^{+2}\right)$ & 2 & 784 & $\mathrm{I}\left(4_{\mathrm{II}}^{+2} 7^{+2}\right)$ & 2 & 800 & $\mathrm{I}\left(2_{1}^{+1} 16_{5}^{-1} 5^{-2}\right)$ \\
\hline 800 & $\mathrm{I}\left(2_{1}^{+1} 16_{3}^{-1} 5^{-2}\right)$ & 2 & 800 & $\mathrm{I}\left(2_{1}^{+1} 16_{1}^{+1} 5^{-2}\right)$ & 2 & 800 & $\mathrm{I}\left(2_{1}^{+1} 16_{7}^{+1} 5^{-2}\right)$ \\
\hline 816 & $\mathrm{I}\left(4_{\mathrm{II}}^{-2} 3^{-1} 17^{+1}\right)$ & 2 & 825 & $\mathrm{I}\left(3^{-1} 5^{-2} 11^{-1}\right)$ & 2 & 828 & $\operatorname{II}\left(4_{5}^{-1} 3^{+2} 23^{+1}\right)$ \\
\hline 845 & $\mathrm{I}\left(5^{-1} 13^{-2}\right)$ & 2 & 847 & $\mathrm{I}\left(7^{+1} 11^{+2}\right)$ & 3 & 864 & $\mathrm{I}\left(2_{1}^{+1} 16_{7}^{+1} 3^{-1} 9^{+1}\right)$ \\
\hline 864 & $\mathrm{I}\left(2_{1}^{+1} 16_{1}^{+1} 3^{+1} 9^{+1}\right)$ & 2 & 864 & $\mathrm{I}\left(2_{1}^{+1} 16_{1}^{+1} 3^{+1} 9^{-1}\right)$ & 2 & 864 & $\mathrm{I}\left(2_{1}^{+1} 16_{7}^{+1} 3^{+1} 9^{+1}\right)$ \\
\hline 864 & $\mathrm{I}\left(2_{1}^{+1} 16_{7}^{+1} 3^{+1} 9^{-1}\right)$ & 2 & 864 & $\mathrm{I}\left(2_{1}^{+1} 16_{1}^{+1} 3^{-1} 9^{+1}\right)$ & 2 & 864 & $\mathrm{I}\left(2_{1}^{+1} 16_{3}^{-1} 3^{+1} 9^{+1}\right)$ \\
\hline 864 & $\mathrm{I}\left(2_{1}^{+1} 16_{3}^{-1} 3^{+1} 9^{-1}\right)$ & 2 & 864 & $\mathrm{I}\left(2_{1}^{+1} 16_{5}^{-1} 3^{-1} 9^{+1}\right)$ & 2 & 864 & $\mathrm{I}\left(2_{1}^{+1} 16_{3}^{-1} 3^{-1} 9^{+1}\right)$ \\
\hline 864 & $\mathrm{I}\left(2_{1}^{+1} 16_{5}^{-1} 3^{+1} 9^{+1}\right)$ & 2 & 864 & $\mathrm{I}\left(2_{1}^{+1} 16_{5}^{-1} 3^{+1} 9^{-1}\right)$ & 2 & 867 & $\mathrm{I}\left(3^{+1} 17^{-2}\right)$ \\
\hline 882 & $\mathrm{II}\left(2_{7}^{+1} 3^{+2} 7^{-2}\right)$ & 2 & 882 & $\mathrm{II}\left(2_{7}^{+1} 9^{-1} 7^{+2}\right)$ & 3 & 882 & $\mathrm{I}\left(2_{1}^{+1} 3^{+2} 7^{+2}\right)$ \\
\hline 882 & $\mathrm{I}\left(2_{1}^{+1} 3^{+2} 7^{-2}\right)$ & 2 & 900 & $\mathrm{I}\left(2_{2}^{+2} 3^{+2} 5^{-2}\right)$ & 1 & 900 & $\mathrm{I}\left(2_{2}^{+2} 3^{-2} 5^{-2}\right)$ \\
\hline 900 & $\mathrm{I}\left(2_{2}^{+2} 3^{+2} 5^{+2}\right)$ & 2 & 900 & $\mathrm{I}\left(2_{\mathrm{II}}^{+2} 3^{-2} 5^{-2}\right)$ & 2 & 900 & $\mathrm{I}\left(2_{\mathrm{II}}^{+2} 3^{+2} 5^{+2}\right)$ \\
\hline 900 & $\mathrm{II}\left(4_{3}^{-1} 3^{-2} 5^{+2}\right)$ & 1 & 900 & $\mathrm{II}\left(4_{3}^{-1} 3^{+2} 5^{-2}\right)$ & 1 & 900 & $\mathrm{II}\left(4_{3}^{-1} 9^{+1} 5^{+2}\right)$ \\
\hline 900 & $\operatorname{II}\left(4_{7}^{+1} 3^{-2} 5^{-2}\right)$ & 2 & 900 & $\operatorname{II}\left(4_{7}^{+1} 3^{+2} 5^{+2}\right)$ & 2 & 900 & $\mathrm{I}\left(4_{1}^{+1} 3^{+2} 5^{-2}\right)$ \\
\hline 900 & $\mathrm{I}\left(4_{1}^{+1} 3^{-2} 5^{-2}\right)$ & 2 & 900 & $\mathrm{I}\left(4_{1}^{+1} 3^{+2} 5^{+2}\right)$ & 2 & 912 & $\mathrm{I}\left(4_{\mathrm{II}}^{-2} 3^{+1} 19^{-1}\right)$ \\
\hline 945 & $\mathrm{I}\left(3^{-1} 9^{-1} 5^{+1} 7^{+1}\right)$ & 3 & 945 & $\mathrm{I}\left(3^{+1} 9^{+1} 5^{-1} 7^{+1}\right)$ & 2 & 945 & $\mathrm{I}\left(3^{+1} 9^{-1} 5^{-1} 7^{+1}\right)$ \\
\hline 975 & $\mathrm{I}\left(3^{+1} 5^{-2} 13^{+1}\right)$ & 3 & 980 & $\operatorname{II}\left(4_{7}^{+1} 5^{-1} 7^{+2}\right)$ & 1 & 980 & $\operatorname{II}\left(4_{7}^{+1} 5^{+1} 7^{-2}\right)$ \\
\hline 990 & $\mathrm{I}\left(2_{1}^{+1} 3^{+2} 5^{+1} 11^{-1}\right)$ & 4 & 1008 & $\mathrm{I}\left(4_{\mathrm{II}}^{-2} 3^{-2} 7^{-1}\right)$ & 2 & 1008 & $\mathrm{I}\left(4_{\mathrm{II}}^{-2} 3^{+2} 7^{+1}\right)$ \\
\hline 1008 & $\mathrm{I}\left(4_{\mathrm{II}}^{+2} 3^{+2} 7^{-1}\right)$ & 2 & 1008 & $\mathrm{I}\left(4_{2}^{+2} 3^{+2} 7^{-1}\right)$ & 2 & 1008 & $\mathrm{I}\left(4_{6}^{+2} 3^{+2} 7^{+1}\right)$ \\
\hline 1008 & $\mathrm{I}\left(16_{3}^{-1} 3^{+2} 7^{+1}\right)$ & 3 & 1008 & $\mathrm{I}\left(16_{7}^{+1} 3^{+2} 7^{+1}\right)$ & 4 & 1014 & $\mathrm{II}\left(2_{1}^{+1} 3^{-1} 13^{+2}\right)$ \\
\hline 1029 & $\mathrm{I}\left(3^{+1} 7^{-1} 49^{+1}\right)$ & 2 & 1040 & $\mathrm{I}\left(4_{\mathrm{II}}^{-2} 5^{+1} 13^{+1}\right)$ & 2 & 1050 & $\mathrm{II}\left(2_{7}^{+1} 3^{-1} 5^{-2} 7^{-1}\right)$ \\
\hline 1050 & $\mathrm{I}\left(2_{1}^{+1} 3^{-1} 5^{-2} 7^{-1}\right)$ & 3 & 1080 & $\mathrm{I}\left(2_{1}^{+1} 4_{1}^{+1} 3^{+1} 9^{+1} 5^{-1}\right)$ & 4 & 1083 & $\mathrm{I}\left(3^{+1} 19^{+2}\right)$ \\
\hline 1089 & $\mathrm{I}\left(3^{-2} 11^{-2}\right)$ & 2 & 1089 & $\mathrm{I}\left(3^{+2} 11^{+2}\right)$ & 2 & 1104 & $\mathrm{I}\left(4_{\mathrm{II}}^{-2} 3^{-1} 23^{+1}\right)$ \\
\hline 1125 & $\mathrm{I}\left(3^{+2} 5^{+1} 25^{-1}\right)$ & 3 & 1125 & $\mathrm{I}\left(3^{+2} 5^{-1} 25^{+1}\right)$ & 1 & 1125 & $\mathrm{I}\left(3^{+2} 5^{-1} 25^{-1}\right)$ \\
\hline 1125 & $\mathrm{I}\left(3^{-2} 5^{+1} 25^{+1}\right)$ & 2 & 1125 & $\mathrm{I}\left(9^{-1} 5^{+1} 25^{-1}\right)$ & 3 & 1134 & $\operatorname{II}\left(2_{1}^{+1} 9^{+2} 7^{+1}\right)$ \\
\hline 1152 & $\mathrm{I}\left(2_{1}^{+1} 64_{3}^{-1} 3^{+2}\right)$ & 4 & 1156 & $\mathrm{II}\left(4_{3}^{-1} 17^{+2}\right)$ & 2 & 1188 & $\operatorname{II}\left(4_{3}^{-1} 3^{-1} 9^{-1} 11^{+1}\right)$ \\
\hline 1188 & $\mathrm{II}\left(4_{3}^{-1} 3^{+1} 9^{+1} 11^{-1}\right)$ & 2 & 1188 & $\mathrm{II}\left(4_{3}^{-1} 3^{+1} 9^{-1} 11^{-1}\right)$ & 2 & 1200 & $\mathrm{I}\left(4_{2}^{+2} 3^{-1} 5^{-2}\right)$ \\
\hline 1200 & $\mathrm{I}\left(4_{2}^{+2} 3^{+1} 5^{+2}\right)$ & 2 & 1200 & $\mathrm{I}\left(4_{6}^{+2} 3^{+1} 5^{-2}\right)$ & 1 & 1200 & $\mathrm{I}\left(4_{\mathrm{II}}^{+2} 3^{-1} 5^{-2}\right)$ \\
\hline 1200 & $\mathrm{I}\left(4_{\mathrm{II}}^{+2} 3^{+1} 5^{+2}\right)$ & 2 & 1200 & $\mathrm{I}\left(4_{\mathrm{II}}^{-2} 3^{-1} 5^{+2}\right)$ & 1 & 1200 & $\mathrm{I}\left(4_{\mathrm{II}}^{-2} 3^{+1} 5^{-2}\right)$ \\
\hline 1200 & $\mathrm{II}\left(16_{1}^{+1} 3^{+1} 5^{-2}\right)$ & 2 & 1225 & $\mathrm{I}\left(5^{-2} 7^{+2}\right)$ & 1 & 1225 & $\mathrm{I}\left(5^{+2} 7^{-2}\right)$ \\
\hline 1232 & $\mathrm{I}\left(4_{\mathrm{II}}^{-2} 7^{+1} 11^{-1}\right)$ & 3 & 1260 & $\mathrm{I}\left(2_{2}^{+2} 3^{+2} 5^{+1} 7^{-1}\right)$ & 3 & 1260 & $\mathrm{I}\left(2_{\mathrm{II}}^{+2} 3^{+2} 5^{+1} 7^{-1}\right)$ \\
\hline 1260 & $\mathrm{II}\left(4_{1}^{+1} 3^{+2} 5^{+1} 7^{+1}\right)$ & 2 & 1260 & $\operatorname{II}\left(4_{1}^{+1} 3^{+2} 5^{-1} 7^{-1}\right)$ & 2 & 1260 & $\mathrm{II}\left(4_{1}^{+1} 3^{-2} 5^{+1} 7^{-1}\right)$ \\
\hline 1260 & $\mathrm{I}\left(4_{7}^{+1} 3^{+2} 5^{+1} 7^{-1}\right)$ & 3 & 1260 & $\operatorname{II}\left(4_{5}^{-1} 3^{+2} 5^{+1} 7^{-1}\right)$ & 3 & 1280 & $\mathrm{I}\left(16_{2}^{+2} 5^{+1}\right)$ \\
\hline 1280 & $\mathrm{I}\left(16_{2}^{-2} 5^{-1}\right)$ & 2 & 1280 & $\mathrm{I}\left(16_{6}^{-2} 5^{+1}\right)$ & 3 & 1296 & $\mathrm{I}\left(4_{\mathrm{II}}^{-2} 3^{+1} 27^{-1}\right)$ \\
\hline 1296 & $\mathrm{I}\left(4_{\mathrm{II}}^{-2} 9^{-2}\right)$ & 1 & 1296 & $\mathrm{I}\left(4_{\mathrm{II}}^{-2} 9^{+2}\right)$ & 1 & 1296 & $\mathrm{I}\left(4_{2}^{+2} 9^{+2}\right)$ \\
\hline 1296 & $\mathrm{I}\left(16_{5}^{-1} 9^{+2}\right)$ & 3 & 1296 & $\mathrm{I}\left(16_{1}^{+1} 9^{+2}\right)$ & 4 & 1300 & $\mathrm{II}\left(4_{7}^{+1} 5^{-2} 13^{-1}\right)$ \\
\hline 1323 & $\mathrm{I}\left(3^{-1} 9^{+1} 7^{-2}\right)$ & 2 & 1323 & $\mathrm{I}\left(3^{+1} 9^{-1} 7^{+2}\right)$ & 1 & 1323 & $\mathrm{I}\left(3^{-1} 9^{-1} 7^{-2}\right)$ \\
\hline 1323 & $\mathrm{I}\left(3^{+1} 9^{+1} 7^{+2}\right)$ & 1 & 1344 & $\mathrm{I}\left(4_{1}^{+1} 16_{1}^{+1} 3^{+1} 7^{-1}\right)$ & 6 & 1350 & $\operatorname{II}\left(2_{1}^{+1} 3^{+1} 9^{+1} 5^{-2}\right)$ \\
\hline
\end{tabular}




\begin{tabular}{|c|c|c|c|c|c|c|c|}
\hline 350 & $\mathrm{II}\left(2_{1}^{+1} 3^{-1} 9^{+1} 5^{+2}\right)$ & 2 & 1350 & $\operatorname{II}\left(2_{1}^{+1} 3^{+1} 9^{-1} 5^{-2}\right)$ & 2 & 1350 & $\mathrm{I}\left(2_{1}^{+1} 3^{+1} 9^{+1} 5^{-2}\right)$ \\
\hline 350 & $\mathrm{I}\left(2_{1}^{+1} 3^{-1} 9^{+1} 5^{+2}\right)$ & 3 & 1350 & $\mathrm{I}\left(2_{1}^{+1} 3^{+1} 9^{-1} 5^{-2}\right)$ & 2 & 1350 & $\mathrm{I}\left(2_{1}^{+1} 3^{-1} 9^{-1} 5^{-2}\right)$ \\
\hline 104 & $\mathrm{I}\left(2_{\mathrm{II}}^{+2} 3^{+1} 9^{+1} 13^{+1}\right)$ & 6 & 1404 & $\mathrm{II}\left(4_{5}^{-1} 3^{+1} 9^{-1} 13^{-1}\right)$ & 2 & 1440 & $\mathrm{I}\left(2_{1}^{+1} 16_{1}^{+1} 3^{+2} 5^{+1}\right)$ \\
\hline 440 & $\mathrm{I}\left(2_{1}^{+1} 16_{7}^{+1} 3^{+2} 5^{+1}\right)$ & 2 & 1440 & $\mathrm{I}\left(2_{1}^{+1} 16_{1}^{+1} 3^{+2} 5^{-1}\right)$ & 3 & 1440 & $\mathrm{I}\left(2_{1}^{+1} 16_{7}^{+1} 3^{+2} 5^{-1}\right)$ \\
\hline 440 & $\mathrm{I}\left(2_{1}^{+1} 16_{5}^{-1} 3^{+2} 5^{-1}\right)$ & 3 & 1440 & $\mathrm{I}\left(2_{1}^{+1} 16_{3}^{-1} 3^{+2} 5^{-1}\right)$ & 3 & 1440 & $\mathrm{I}\left(2_{1}^{+1} 16_{5}^{-1} 3^{+2} 5^{+1}\right)$ \\
\hline 440 & $\mathrm{I}\left(2_{1}^{+1} 16_{3}^{-1} 3^{+2} 5^{+1}\right)$ & 2 & 1452 & $\mathrm{I}\left(2_{2}^{+2} 3^{+1} 11^{+2}\right)$ & 3 & 1452 & $\mathrm{II}\left(4_{1}^{+1} 3^{-1} 11^{-2}\right)$ \\
\hline 452 & $\mathrm{II}\left(4_{1}^{+1} 3^{+1} 11^{+2}\right)$ & 2 & 1452 & $\mathrm{I}\left(4_{7}^{+1} 3^{+1} 11^{+2}\right)$ & 3 & 1470 & $\mathrm{II}\left(2_{1}^{+1} 3^{+1} 5^{-1} 7^{+2}\right)$ \\
\hline 470 & $\mathrm{I}\left(2_{1}^{+1} 3^{+1} 5^{-1} 7^{+2}\right)$ & 3 & 1500 & $\mathrm{I}\left(2_{\mathrm{II}}^{+2} 3^{-1} 5^{-1} 25^{+1}\right)$ & 2 & 1500 & $\mathrm{I}\left(2_{2}^{+2} 3^{-1} 5^{-1} 25^{+1}\right)$ \\
\hline 500 & $\mathrm{II}\left(4_{5}^{-1} 3^{+1} 5^{-1} 25^{-1}\right)$ & 2 & 1500 & $\mathrm{II}\left(4_{5}^{-1} 3^{-1} 5^{+1} 25^{+1}\right)$ & 1 & 1500 & $\left.{ }^{-1} 5^{+1} 25^{-1}\right)$ \\
\hline 500 & $\operatorname{II}\left(4_{1}^{+1} 3^{-1} 5^{-1} 25^{+1}\right)$ & 3 & 1500 & $\mathrm{I}\left(4_{7}^{+1} 3^{-1} 5^{-1} 25^{+1}\right)$ & 3 & 1521 & $\mathrm{I}\left(3^{+2} 13^{-2}\right)$ \\
\hline 568 & $\mathrm{I}\left(2_{1}^{+1} 16_{5}^{-1} 7^{+2}\right)$ & 3 & 1568 & $\mathrm{I}\left(2_{1}^{+1} 16_{3}^{-1} 7^{+2}\right)$ & 3 & 1568 & $\mathrm{I}\left(2_{1}^{+1} 16_{1}^{+1} 7^{+2}\right)$ \\
\hline 568 & $\mathrm{I}\left(2_{1}^{+1} 16_{7}^{+1} 7^{+2}\right)$ & 3 & 1575 & $\mathrm{I}\left(3^{+2} 5^{-2} 7^{+1}\right)$ & 3 & 1575 & \\
\hline 1575 & $\mathrm{I}\left(3^{+}\right.$ & 2 & 1575 & $\left.-27^{-1}\right)$ & 2 & 1584 & $\left.1^{+1}\right)$ \\
\hline 1584 & $\mathrm{I}\left(4_{\mathrm{II}}^{-2} 3^{+2} 11^{+1}\right)$ & 2 & 1584 & $\mathrm{I}\left(4_{\mathrm{II}}^{-2} 3^{-2} 11^{-1}\right)$ & 2 & 1587 & $\mathrm{I}\left(3^{+1}\right.$ \\
\hline 1600 & $\mathrm{I}\left(4_{1}^{+1} 16_{1}^{+1} 5^{+2}\right)$ & 6 & 1620 & $\operatorname{II}\left(4_{7}^{+1} 9^{+2} 5^{+1}\right)$ & 2 & 1650 & $\operatorname{II}\left(2_{7}^{+1} 3^{+1} 5^{-2} 11^{-1}\right)$ \\
\hline 680 & $\mathrm{I}\left(4_{\mathrm{II}}^{-2} 3^{+1} 5^{-1} 7^{+1}\right)$ & 2 & 1680 & $\mathrm{I}\left(4_{\mathrm{II}}^{-2} 3^{-1} 5^{-1} 7^{-1}\right)$ & 2 & 1680 & $\mathrm{I}\left(4_{\mathrm{II}}^{-2} 3^{-1} 5^{+1} 7^{+1}\right)$ \\
\hline 1680 & $\mathrm{I}\left(4_{6}^{+2} 3^{-1} 5^{-1} 7^{+1}\right)$ & 3 & 1680 & $\mathrm{I}\left(4_{\mathrm{II}}^{+2} 3^{-1} 5^{-1} 7^{+1}\right)$ & 3 & 1728 & $\mathrm{I}\left(4_{1}^{+1} 16_{1}^{+1} 3^{+1} 9^{+1}\right)$ \\
\hline 1728 & $\mathrm{I}\left(4_{1}^{+1} 16_{7}^{+1} 3^{+1} 9^{+1}\right)$ & 4 & 1728 & $\mathrm{I}\left(4_{7}^{+1} 16_{1}^{+1} 3^{+1} 9^{+1}\right)$ & 4 & 1764 & $\mathrm{I}\left(2_{2}^{+2} 3^{+2} 7^{+2}\right)$ \\
\hline 764 & $\mathrm{I}\left(2_{2}^{+2} 3^{+2} 7^{-2}\right)$ & 2 & 1764 & $\mathrm{I}\left(2_{\mathrm{II}}^{+2} 3^{+2} 7^{-2}\right)$ & 2 & 1764 & $\mathrm{I}\left(2_{\mathrm{II}}^{+2} 9^{+1} 7^{+2}\right)$ \\
\hline 764 & $\operatorname{II}\left(4_{3}^{-1} 3^{-2} 7^{-2}\right)$ & 2 & 1764 & $\left.3^{+2} 7^{+2}\right)$ & 1 & 1764 & $\left.+27^{-2}\right)$ \\
\hline 1764 & $\left.27^{+2}\right)$ & 2 & 1764 & $\mathrm{I}\left(4_{1}^{+1} 3^{+2} 7^{-2}\right)$ & 2 & 1800 & $\left.{ }_{1}^{+1} 3^{+2} 5^{-2}\right)$ \\
\hline 1800 & $\mathrm{I}\left(2_{1}^{+1} 4_{1}^{+1} 3^{+2} 5^{+2}\right)$ & 3 & 1815 & $\mathrm{I}\left(3^{-1} 5^{+1} 11^{-2}\right)$ & 2 & 1872 & $\mathrm{I}\left(4_{\mathrm{II}}^{-2} 3^{+2} 13^{-1}\right)$ \\
\hline 1890 & $\mathrm{I}\left(2_{7}^{+1} 3^{+1} 9^{-1} 5^{+1} 7^{+1}\right)$ & 4 & 1936 & $\mathrm{I}\left(4_{\mathrm{II}}^{-2} 11^{-2}\right)$ & 1 & 1980 & $\mathrm{I}\left(2_{\mathrm{II}}^{+2} 3^{+2} 5^{-1} 11^{+1}\right)$ \\
\hline 1980 & $\mathrm{II}\left(4_{5}^{-1} 3^{+2} 5^{-1} 11^{-1}\right)$ & 2 & 2000 & $\mathrm{I}\left(4_{2}^{+2} 5^{+1} 25^{+1}\right)$ & 3 & 2000 & $\mathrm{I}\left(4_{\mathrm{II}}^{-2} 5^{+1} 25^{-1}\right)$ \\
\hline 2000 & $\mathrm{I}\left(4_{\mathrm{II}}^{-2} 5^{+1} 25^{+1}\right)$ & 1 & 2000 & $\mathrm{I}\left(16_{1}^{+1} 5^{+1} 25^{+1}\right)$ & 6 & 2000 & \\
\hline 2025 & $\mathrm{I}\left(9^{+2} 5^{+2}\right)$ & 2 & 2028 & $\mathrm{I}\left(2_{\mathrm{II}}^{+2} 3^{+1} 13^{+2}\right)$ & 3 & 2028 & $\mathrm{II}\left(4_{1}^{+1} 3^{+1} 13^{-2}\right)$ \\
\hline 2052 & $\operatorname{II}\left(4_{3}^{-1} 3^{+1} 9^{-1} 19^{-1}\right)$ & 3 & 2100 & $\left.{ }^{1} 5^{-2} 7^{-1}\right)$ & 3 & 2100 & $\mathrm{I}\left(2_{\mathrm{II}}^{+2} 3^{+1} 5^{-2} 7^{-1}\right)$ \\
\hline 2100 & $\mathrm{II}\left(4_{7}^{+1} 3^{-1} 5^{-2} 7^{-1}\right)$ & 2 & 2100 & $\mathrm{II}\left(4_{7}^{+1} 3^{+1} 5^{-2} 7^{+1}\right)$ & 2 & 2100 & $\mathrm{II}\left(4_{7}^{+1} 3^{+1} 5^{+2} 7^{-1}\right)$ \\
\hline 100 & $\mathrm{I}\left(4_{1}^{+1} 3^{+1} 5^{-2} 7^{-1}\right)$ & 3 & 2100 & $\mathrm{II}\left(4_{3}^{-1} 3^{+1} 5^{-2} 7^{-1}\right)$ & 3 & 2156 & $\mathrm{II}\left(4_{1}^{+1} 7^{+2} 11^{+1}\right)$ \\
\hline 160 & $\left.{ }^{-1} 9^{-1} 5^{+1}\right)$ & 2 & 2160 & $\left.{ }^{1} 9^{+1} 5^{-1}\right)$ & 1 & 2160 & $\left.{ }^{+1} 9^{-1} 5^{-1}\right)$ \\
\hline 2160 & $\mathrm{I}\left(4_{\mathrm{II}}^{+2} 3^{+1} 9^{+1} 5^{+1}\right)$ & 2 & 2160 & $\mathrm{I}\left(4_{\mathrm{II}}^{+2} 3^{-1} 9^{+1} 5^{-1}\right)$ & 3 & 2160 & $\mathrm{I}\left(4_{\mathrm{II}}^{+2} 3^{+1} 9^{-1} 5^{+1}\right)$ \\
\hline 2160 & $\mathrm{I}\left(4_{2}^{+2} 3^{+1} 9^{+1} 5^{+1}\right)$ & 2 & 2160 & $\mathrm{I}\left(4_{2}^{+2} 3^{-1} 9^{+1} 5^{-1}\right)$ & 3 & 2160 & $\mathrm{I}\left(4_{2}^{+2} 3^{+1} 9^{-1} 5^{+1}\right)$ \\
\hline 2160 & $\mathrm{I}\left(4_{6}^{+2} 3^{-1} 9^{-1} 5^{+1}\right)$ & 2 & 2160 & $\mathrm{II}\left(16_{5}^{-1} 3^{-1} 9^{-1} 5^{+1}\right)$ & 4 & 2178 & $\mathrm{I}\left(2_{1}^{+1} 3^{+2} 11^{+2}\right)$ \\
\hline 2205 & $\mathrm{I}\left(3^{+2} 5^{-1} 7^{+2}\right)$ & 3 & 2205 & $\mathrm{I}\left(3^{+2} 5^{-1} 7^{-2}\right)$ & 2 & 2205 & $\mathrm{I}\left(3^{+2} 5^{+1} 7^{+2}\right)$ \\
\hline 2205 & $\mathrm{I}\left(3^{-2} 5^{-1} 7^{+2}\right)$ & 2 & 2250 & $\mathrm{II}\left(2_{7}^{+1} 3^{+2} 5^{-1} 25^{+1}\right)$ & 2 & 2250 & $\mathrm{I}\left(2_{1}^{+1} 3^{+2} 5^{-1} 25^{+1}\right)$ \\
\hline 2268 & $\mathrm{I}\left(2_{\mathrm{II}}^{+2} 9^{+2} 7^{+1}\right)$ & 3 & 2304 & $\mathrm{I}\left(16_{\mathrm{II}}^{-2} 3^{-2}\right)$ & 2 & 2304 & $\mathrm{I}\left(16_{4}^{-2} 3^{+2}\right)$ \\
\hline 2304 & $\mathrm{I}\left(16_{2}^{-2} 3^{+2}\right)$ & 1 & 2304 & $\mathrm{I}\left(16_{6}^{-2} 9^{+1}\right)$ & 3 & 2304 & $\mathrm{I}\left(16_{2}^{+2} 9^{+1}\right)$ \\
\hline 2304 & $\mathrm{I}\left(16_{6}^{+2} 3^{+2}\right)$ & 2 & 2304 & $\mathrm{I}\left(16_{0}^{+2} 3^{+2}\right)$ & 2 & 2340 & $\operatorname{II}\left(4_{3}^{-1} 3^{+2} 5^{+1} 13^{-1}\right)$ \\
\hline 352 & $\mathrm{I}\left(4_{2}^{+2} 3^{+1} 7^{-2}\right)$ & 2 & 2352 & $\mathrm{I}\left(4_{6}^{+2} 3^{+1} 7^{+2}\right)$ & 2 & 2352 & $\mathrm{I}\left(4_{\mathrm{II}}^{+2} 3^{+1} 7^{-2}\right)$ \\
\hline
\end{tabular}




\begin{tabular}{|c|c|c|c|c|c|c|c|}
\hline 352 & $\mathrm{I}\left(4_{\mathrm{II}}^{-2} 3^{-1} 7^{-2}\right)$ & 2 & 2352 & $\mathrm{I}\left(4_{\mathrm{II}}^{-2} 3^{+1} 7^{+2}\right)$ & 1 & 2352 & $\mathrm{I}\left(16_{7}^{+1} 3^{+1} 7^{+2}\right)$ \\
\hline 52 & $\mathrm{I}\left(16_{3}^{-1} 3^{+1} 7^{+2}\right)$ & & 2400 & $\mathrm{I}\left(2_{1}^{+1} 16_{7}^{+1} 3^{-1} 5^{+2}\right)$ & 3 & 2400 & $\mathrm{I}\left(2_{1}^{+1} 16_{1}^{+1} 3^{-1} 5^{-2}\right)$ \\
\hline & $\mathrm{I}\left(2_{1}^{+1} 16_{7}^{+1} 3^{-1} 5^{-2}\right)$ & & 2400 & $\mathrm{I}\left(2_{1}^{+1} 16_{1}^{+1} 3^{-1} 5^{+2}\right)$ & 3 & 2400 & $\mathrm{I}\left(2_{1}^{+1} 16_{3}^{-1} 3^{-1} 5^{-2}\right)$ \\
\hline & $\mathrm{I}\left(2_{1}^{+1} 16_{5}^{-1} 3^{-1} 5^{+2}\right)$ & 3 & 2400 & $\mathrm{I}\left(2_{1}^{+1} 16_{3}^{-1} 3^{-1} 5^{+2}\right)$ & 3 & 2400 & $\mathrm{I}\left(2_{1}^{+1} 16_{5}^{-1} 3^{-1} 5^{-2}\right)$ \\
\hline 48 & $\mathrm{I}\left(4_{\mathrm{II}}^{-2} 3^{+2} 17^{-1}\right)$ & 2 & 2475 & $\mathrm{I}\left(3^{+2} 5^{-2} 11^{-1}\right)$ & 2 & 2535 & $\mathrm{I}\left(3^{-1} 5^{+1} 13^{+2}\right)$ \\
\hline 01 & $\mathrm{I}\left(3^{+2} 17^{-2}\right)$ & 2 & 2625 & $\mathrm{I}\left(3^{-1} 5^{+1} 25^{+1} 7^{+1}\right)$ & 3 & 2640 & $\mathrm{I}\left(4_{\mathrm{II}}^{-2} 3^{+1} 5^{+1} 11^{-1}\right)$ \\
\hline & $\mathrm{II}\left(2_{1}^{+1} 3^{-1} 9^{-1} 7^{-2}\right)$ & & 2646 & $\mathrm{I}\left(2_{1}^{+1} 3^{-1} 9^{-1} 7^{+2}\right)$ & 3 & 2700 & $\mathrm{I}\left(2_{2}^{+2} 3^{-1} 9^{+1} 5^{-2}\right)$ \\
\hline 00 & $\mathrm{I}\left(2_{2}^{+2} 3^{+1} 9^{-1} 5^{+2}\right)$ & 3 & 2700 & $\mathrm{I}\left(2_{2}^{+2} 3^{-1} 9^{-1} 5^{-2}\right)$ & 2 & 2700 & $\mathrm{I}\left(2_{\mathrm{II}}^{+2} 3^{-1} 9^{+1} 5^{-2}\right)$ \\
\hline 00 & $\mathrm{I}\left(2_{\mathrm{II}}^{+2} 3^{+1} 9^{-1} 5^{+2}\right)$ & & 2700 & $\mathrm{I}\left(2_{\mathrm{II}}^{+2} 3^{-1} 9^{-1} 5^{-2}\right)$ & 2 & 2700 & $\left.{ }^{1} 5^{-2}\right)$ \\
\hline 00 & $\operatorname{II}\left(4_{1}^{+1} 3^{-1} 9^{+1} 5^{+2}\right)$ & & 2700 & $\mathrm{II}\left(4_{1}^{+1} 3^{-1} 9^{-1} 5^{+2}\right)$ & 1 & 2700 & $\mathrm{II}\left(4_{1}^{+1} 3^{+1} 9^{+1} 5^{-2}\right)$ \\
\hline & $\mathrm{I}\left(4_{7}^{+1} 3^{+1} 9^{+1} 5^{-2}\right)$ & 2 & 2700 & $\mathrm{I}\left(4_{7}^{+1} 3^{-1} 9^{+1} 5^{-2}\right)$ & 2 & 2700 & \\
\hline & $\left.{ }^{-1} 9^{-1} 5^{-2}\right)$ & 2 & 2700 & $\operatorname{II}\left(4_{5}^{-1} 3^{-1} 9^{+1} 5^{-2}\right)$ & 2 & 2700 & $\left.{ }^{+1} 9^{-1} 5^{+2}\right)$ \\
\hline 00 & $\operatorname{II}\left(4_{5}^{-1} 3^{-1} 9^{-1} 5^{-2}\right)$ & 2 & 2736 & $\mathrm{I}\left(4_{\mathrm{II}}^{-2} 3^{+2} 19^{+1}\right)$ & 2 & 2800 & $\left.5^{+2} 7^{-1}\right)$ \\
\hline 00 & $\mathrm{I}\left(4_{\mathrm{II}}^{-2} 5^{-2} 7^{+1}\right)$ & & 2925 & $\mathrm{I}\left(3^{+2} 5^{-2} 13^{+1}\right)$ & 3 & 2940 & $\left.5^{+1} 7^{+2}\right)$ \\
\hline 10 & $\mathrm{I}\left(2_{2}^{+2} 3^{-}\right.$ & 3 & 2940 & $\mathrm{II}\left(4_{5}^{-1} 3^{+1} 5^{+1} 7^{+2}\right)$ & 2 & 2940 & $\left.7^{-2}\right)$ \\
\hline 40 & $\left.5^{-1} 7^{+2}\right)$ & 2 & 2940 & $\left.5^{+1} 7^{+2}\right)$ & 3 & 2940 & $\left.{ }^{1} 7^{+2}\right)$ \\
\hline 24 & $\left.{ }^{1} 9^{+1} 7^{-1}\right)$ & 3 & 3024 & $\mathrm{I}\left(4_{\mathrm{II}}^{-2} 3^{-1} 9^{+1} 7^{+1}\right)$ & 2 & 3024 & $\left.{ }^{1} 9^{-1} 7^{-1}\right)$ \\
\hline 24 & $\mathrm{I}\left(4_{\mathrm{II}}^{-2} 3^{-1} 9^{-1} 7^{+1}\right)$ & 2 & 3024 & $\mathrm{I}\left(4_{\mathrm{II}}^{-2} 3^{+1} 9^{+1} 7^{-1}\right)$ & 1 & 3024 & $\mathrm{I}\left(16_{1}^{+1} 3^{+1} 9^{+1} 7^{-1}\right)$ \\
\hline 24 & $\left.3^{+1} 9^{+1} 7^{-1}\right)$ & 6 & 3042 & $\operatorname{II}\left(2_{7}^{+1} 3^{+2} 13^{+2}\right)$ & 3 & 3087 & $\left.{ }^{+1} 49^{-1}\right)$ \\
\hline 20 & $\left.{ }^{-1} 13^{-1}\right)$ & 3 & 3150 & $\left.5^{-2} 7^{+1}\right)$ & 2 & 3150 & \\
\hline & $\mathrm{I}\left(3^{+2} 19^{+2}\right)$ & 2 & 3267 & $\left.{ }^{+1} 11^{-2}\right)$ & 2 & 3267 & \\
\hline & $\left.11^{+2}\right)$ & 3 & 3300 & $\left.-211^{+1}\right)$ & 4 & 3300 & $\mathrm{II}(4$ \\
\hline 312 & $\left.23^{+1}\right)$ & 3 & 3375 & $\mathrm{I}\left(3^{-1} 9^{+1} 5^{-1} 25^{+1}\right)$ & 6 & 3375 & $\left.5^{+1} 25^{+1}\right)$ \\
\hline 775 & $\mathrm{I}\left(3^{-1} 9^{-1} 5^{+1} 25^{-1}\right)$ & 2 & 3375 & $\mathrm{I}\left(3^{+1} 9^{+1} 5^{-1} 25^{-1}\right)$ & 2 & 3375 & $\mathrm{I}\left(3^{+1} 9^{-1} 5^{-1} 25^{-1}\right)$ \\
\hline & $\operatorname{II}\left(4_{7}^{+1} 5^{-1} 13^{-2}\right)$ & 2 & 3388 & $\operatorname{II}\left(4_{5}^{-1} 7^{+1} 11^{+2}\right)$ & 3 & 3456 & $\mathrm{I}\left(2_{1}^{+}\right.$ \\
\hline 168 & $\left.{ }^{1} 17^{-2}\right)$ & 2 & 3600 & $\left.{ }^{2} 5^{+2}\right)$ & 1 & 3600 & $\left.3^{+2} 5^{-2}\right)$ \\
\hline 600 & $\left.{ }^{1} 5^{+2}\right)$ & 2 & 3600 & $\left.5^{-2}\right)$ & 1 & 3600 & $\left.5^{-2}\right)$ \\
\hline 00 & & 2 & 3600 & & 2 & 3600 & $\left.3^{+2}\right)$ \\
\hline 00 & $\left.+25^{-2}\right)$ & 2 & 3630 & $\left.-11^{+2}\right)$ & 4 & 3675 & $\left.-27^{+2}\right)$ \\
\hline 675 & $\left.7^{+2}\right)$ & 2 & 3675 & $\left.-27^{-2}\right)$ & 2 & 3675 & $\left.-27^{+2}\right)$ \\
\hline 80 & $\left.9^{+1} 5^{-1} 7^{+1}\right)$ & 4 & 3780 & $\left(4_{3}^{-1} 3^{-1} 9^{-1} 5^{+1} 7^{+1}\right)$ & 3 & 3780 & $\left.9^{+1} 5^{-1} 7^{+1}\right)$ \\
\hline & $\left(4_{3}^{-1} 3^{+1} 9^{-1} 5^{-1} 7^{+1}\right)$ & 2 & 3840 & $\mathrm{I}\left(16_{\mathrm{II}}^{-2} 3^{-1} 5^{+1}\right)$ & 2 & 3900 & $\operatorname{II}\left(4_{5}^{-1} 3^{+1} 5^{-2} 13^{+1}\right)$ \\
\hline & $\mathrm{I}\left(4_{\mathrm{II}}^{-2} 5^{-1} 7^{+2}\right)$ & 1 & 3920 & $\mathrm{I}\left(4_{\mathrm{II}}^{-2} 5^{+1} 7^{-2}\right)$ & 2 & 4032 & $\mathrm{I}\left(4_{7}^{+1} 16_{7}^{+1} 3^{+2} 7^{+1}\right)$ \\
\hline 11 & $\left(4_{7}^{+1} 3^{+1} 7^{-1} 49^{+1}\right)$ & 2 & 4225 & $\mathrm{I}\left(5^{-2} 13^{-2}\right)$ & 2 & 4320 & $\left.3^{+1} 9^{+1} 5^{-1}\right)$ \\
\hline 20 & $\mathrm{I}\left(2_{1}^{+1} 16_{5}^{-1} 3^{+1} 9^{+1} 5^{-1}\right)$ & 4 & 4320 & $\mathrm{I}\left(2_{1}^{+1} 16_{1}^{+1} 3^{+1} 9^{+1} 5^{-1}\right)$ & 4 & 4320 & $\mathrm{I}\left(2_{1}^{+1} 1\right.$ \\
\hline 32 & $\mathrm{II}\left(4_{1}^{+1} 3^{+1} 19^{+2}\right)$ & 2 & 4356 & $\mathrm{I}\left(2_{2}^{+2} 3^{+2} 11^{+2}\right)$ & 3 & 4356 & $\left.3^{-2} 11^{-2}\right)$ \\
\hline 435 & $\left.+211^{+2}\right)$ & 2 & 4356 & $\mathrm{I}\left(4_{1}^{+1} 3^{+2} 11^{+2}\right)$ & 3 & 4410 & $\left.{ }^{+2} 5^{+1} 7^{+2}\right)$ \\
\hline & $\left.{ }^{2} 5^{+1} 7^{+2}\right)$ & 3 & 4500 & $\mathrm{I}\left(2_{2}^{+2} 3^{+2} 5^{+1} 25^{-1}\right)$ & 3 & 4500 & $\left.{ }^{+2} 5^{+1} 25^{-1}\right)$ \\
\hline & $\left.{ }^{2} 5^{-1} 25^{+1}\right)$ & 1 & 4500 & $\mathrm{II}\left(4_{7}^{+1} 3^{+2} 5^{-1} 25^{-1}\right)$ & 1 & 4500 & $\left.{ }^{-2} 5^{+1} 25^{+1}\right)$ \\
\hline
\end{tabular}




\begin{tabular}{|c|c|c|c|c|c|c|c|}
\hline 500 & $\mathrm{II}\left(4_{7}^{+1} 9^{-1} 5^{+1} 25^{-1}\right)$ & 3 & 1500 & $\mathrm{I}\left(4_{1}^{+1} 3^{+2} 5^{+1} 25^{-1}\right)$ & 3 & 4500 & $\mathrm{II}\left(4_{3}^{-1} 3^{+2} 5^{+1} 25^{-1}\right)$ \\
\hline 63 & $\mathrm{I}\left(3^{+1} 9^{-1} 13^{-2}\right)$ & 2 & 4624 & $\mathrm{I}\left(4_{\mathrm{II}}^{-2} 17^{+2}\right)$ & 2 & 4725 & $\mathrm{I}\left(3^{-1} 9^{+1} 5^{-2} 7^{-1}\right)$ \\
\hline 25 & $\mathrm{I}\left(3^{-1} 9^{-1} 5^{-2} 7^{-1}\right)$ & 2 & 1725 & $\mathrm{I}\left(3^{+1} 9^{+1} 5^{+2} 7^{-1}\right)$ & 3 & 4752 & $\mathrm{I}\left(4_{\mathrm{II}}^{-2} 3^{-1} 9^{-1} 11^{+1}\right)$ \\
\hline 52 & $\mathrm{I}\left(4_{\mathrm{II}}^{-2} 3^{+1} 9^{+1} 11^{-1}\right)$ & 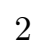 & 4752 & $\mathrm{I}\left(4_{\mathrm{II}}^{-2} 3^{+1} 9^{-1} 11^{-1}\right)$ & 2 & 4761 & $\mathrm{I}\left(3^{+2} 23^{+2}\right)$ \\
\hline 900 & $\mathrm{II}\left(4_{3}^{-1} 5^{-2} 7^{+2}\right)$ & 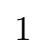 & 4900 & $\mathrm{II}\left(4_{3}^{-1} 5^{+2} 7^{-2}\right)$ & 2 & 4950 & $\mathrm{II}\left(2_{1}^{+1} 3^{+2} 5^{-2} 11^{-1}\right)$ \\
\hline 40 & $\mathrm{I}\left(4_{2}^{+2} 3^{+2} 5^{+1} 7^{-1}\right)$ & 3 & 5040 & $\mathrm{I}\left(4_{\mathrm{II}}^{+2} 3^{+2} 5^{+1} 7^{-1}\right)$ & 3 & 5040 & $\mathrm{I}\left(4_{\mathrm{II}}^{-2} 3^{+2} 5^{+1} 7^{+1}\right)$ \\
\hline 40 & $\mathrm{I}\left(4_{\mathrm{II}}^{-2} 3^{+2} 5^{-1} 7^{-1}\right)$ & 2 & 5040 & $\mathrm{I}\left(4_{\mathrm{II}}^{-2} 3^{-2} 5^{+1} 7^{-1}\right)$ & 2 & 5184 & $\left.3_{1}^{+1} 9^{+2}\right)$ \\
\hline 00 & $\mathrm{I}\left(4_{\mathrm{II}}^{-2} 5^{-2} 13^{-1}\right)$ & 2 & 5292 & $\mathrm{I}\left(2_{\mathrm{II}}^{+2} 3^{+1} 9^{+1} 7^{-2}\right)$ & 4 & 5292 & $\left.+17^{+2}\right)$ \\
\hline 292 & $\mathrm{II}\left(4_{1}^{+1} 3^{-1} 9^{+1} 7^{-2}\right)$ & 2 & 5292 & $\operatorname{II}\left(4_{1}^{+1} 3^{+1} 9^{-1} 7^{+2}\right)$ & 1 & 5292 & $\left.9^{-1} 7^{-2}\right)$ \\
\hline 292 & $\mathrm{II}\left(4_{1}^{+1} 3^{+1} 9^{+1} 7^{+2}\right)$ & 1 & 5292 & $\mathrm{I}\left(4_{7}^{+1} 3^{+1} 9^{+1} 7^{+2}\right)$ & 3 & 5376 & $\mathrm{I}\left(16_{2}^{+2} 3^{+1} 7^{-1}\right)$ \\
\hline 376 & $\mathrm{I}\left(16_{6}^{-2} 3^{+1} 7^{-1}\right)$ & 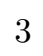 & 5400 & $\mathrm{I}\left(2_{1}^{+1} 4_{1}^{+1} 3^{-1} 9^{-1} 5^{-2}\right)$ & 4 & 5445 & $\left.{ }^{-1} 11^{-2}\right)$ \\
\hline 16 & $\left.{ }^{-1} 13^{-1}\right)$ & 2 & 5808 & $\mathrm{I}\left(4_{6}^{+2} 3^{+1} 11^{+2}\right)$ & 3 & 5808 & $\left.1^{-2}\right)$ \\
\hline 308 & $\left.{ }^{1} 11^{+2}\right)$ & 2 & 5929 & $\mathrm{I}\left(7^{+2} 11^{+2}\right)$ & 3 & 6000 & $\left.{ }^{-1} 25^{-1}\right)$ \\
\hline 000 & $\mathrm{I}\left(4_{\mathrm{II}}^{-2} 3^{-1} 5^{+1} 25^{+1}\right)$ & 1 & 6000 & $\mathrm{I}\left(4_{\mathrm{II}}^{-2} 3^{-1} 5^{+1} 25^{-1}\right)$ & 1 & 6000 & $\mathrm{I}\left(4_{\mathrm{II}}^{+2} 3^{-1} 5^{-1} 25^{+1}\right)$ \\
\hline 00 & $\left.5^{-1} 25^{+1}\right)$ & 3 & 6084 & $\mathrm{I}\left(2_{\mathrm{II}}^{+2} 3^{+2} 13^{+2}\right)$ & 3 & 6084 & $\mathrm{II}\left(4_{3}^{-1} 3^{+2} 13^{-2}\right)$ \\
\hline 300 & $\left.5^{-2} 7^{+1}\right)$ & 2 & 6300 & $\left.5^{-2} 7^{+1}\right)$ & 3 & 6300 & \\
\hline 00 & $\left.5^{+2} 7^{+1}\right)$ & 2 & 6300 & $\left.+25^{-2} 7^{-1}\right)$ & 2 & 6300 & $\left.5^{-2} 7^{+1}\right)$ \\
\hline 300 & $\mathrm{I}\left(4_{7}^{+1} 3^{+2} 5^{-2} 7^{+1}\right)$ & 3 & 6348 & $\mathrm{II}\left(4_{1}^{+1} 3^{+1} 23^{+2}\right)$ & 3 & 6400 & $\mathrm{I}\left(16_{2}^{-2} 5^{-2}\right)$ \\
\hline 100 & & 3 & 6400 & & 4 & 6480 & $\left.25^{+1}\right)$ \\
\hline 15 & $\left.-17^{+2}\right)$ & 3 & 6615 & $\left.5^{+1} 7^{+2}\right)$ & 2 & 6615 & $\left.+17^{+2}\right)$ \\
\hline 50 & $\left.{ }^{1} 5^{+1} 25^{-1}\right)$ & 4 & 6750 & $\mathrm{I}\left(2_{1}^{+1} 3^{+1} 9^{-1} 5^{+1} 25^{-1}\right)$ & 6 & 6912 & $\left.{ }^{1} 9^{-1}\right)$ \\
\hline 12 & $\mathrm{I}\left(16_{2}^{+2} 3^{+1} 9^{+1}\right)$ & 2 & 6912 & $\mathrm{I}\left(16_{0}^{+2} 3^{+1} 9^{+1}\right)$ & 4 & 6912 & $\left.{ }^{-1} 9^{+1}\right)$ \\
\hline 912 & $\left.{ }^{-1} 9^{-1}\right)$ & 2 & 6912 & $\left.3^{+1} 9^{+1}\right)$ & 4 & 6912 & $\left.{ }^{1} 9^{+1}\right)$ \\
\hline 566 & $\left.-27^{-2}\right)$ & 2 & 7056 & $\mathrm{I}\left(4_{\mathrm{II}}^{-2} 3^{+2} 7^{+2}\right)$ & 1 & 7056 & $\left.27^{+2}\right)$ \\
\hline 56 & $\left.-27^{-2}\right)$ & 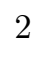 & 7056 & $\left.+27^{-2}\right)$ & 2 & 7056 & $\left.3^{+2} 7^{+2}\right)$ \\
\hline 56 & $\left.{ }^{2} 7^{+2}\right)$ & 4 & 7200 & $\left.{ }^{1} 3^{+2} 5^{+2}\right)$ & 3 & 7200 & $\left.{ }^{1} 3^{+2} 5^{+2}\right)$ \\
\hline 200 & $\left.+25^{-2}\right)$ & 2 & 7200 & $\left.3^{+2} 5^{-2}\right)$ & 2 & 7200 & $\left.3^{+2} 5^{-2}\right)$ \\
\hline 200 & $\left.3^{+2} 5^{-2}\right)$ & 2 & 7200 & $\mathrm{I}\left(2_{1}^{+1} 1\right.$ & 3 & 7200 & $\mathrm{I}\left(2_{1}^{+1}\right.$ \\
\hline 260 & $\mathrm{I}\left(2_{\mathrm{II}}^{+2} 3^{-1} 5^{+1} 11^{+2}\right)$ & 4 & 7260 & $\left.5^{+1} 11^{-2}\right)$ & 2 & 7350 & $\left.{ }^{1} 5^{-2} 7^{+2}\right)$ \\
\hline 50 & $\left.5^{-2} 7^{+2}\right)$ & 3 & 7605 & $\left.{ }^{-1} 13^{+2}\right)$ & 3 & 7875 & $\mathrm{I}\left(3^{+2} 5^{-1} 25^{-1} 7^{-1}\right)$ \\
\hline 920 & $\left.-111^{-1}\right)$ & 2 & 7938 & $\left.+27^{+2}\right)$ & 3 & 8000 & $\left.{ }^{+1} 25^{+1}\right)$ \\
\hline 8100 & $\left.9^{+2} 5^{+2}\right)$ & 2 & 8112 & & 1 & 8208 & $\mathrm{I}\left(4_{\mathrm{II}}^{-2} 3^{+1} 9^{-1} 19^{-1}\right)$ \\
\hline 100 & $\mathrm{I}\left(4_{\mathrm{II}}^{+2} 3^{+1} 5^{-2} 7^{-1}\right)$ & 3 & 8400 & $\mathrm{I}\left(4_{6}^{+2} 3^{+1} 5^{-2} 7^{-1}\right)$ & 3 & 8400 & $\mathrm{I}\left(4_{\mathrm{II}}^{-2} 3^{-1} 5^{-2} 7^{-1}\right)$ \\
\hline 100 & $\left.5^{-2} 7^{+1}\right)$ & ? & 8400 & $\left.{ }^{-1} 5^{+2} 7^{-1}\right)$ & 2 & 8624 & $\mathrm{I}\left(4_{\mathrm{II}}^{-2} 7^{+2} 11^{+1}\right)$ \\
\hline 820 & $\left.5^{-1} 7^{+2}\right)$ & 3 & 8820 & $\left.{ }^{2} 5^{-1} 7^{+2}\right)$ & 2 & 8820 & $\left.5^{-1} 7^{-2}\right)$ \\
\hline 820 & $\mathrm{II}\left(4_{7}^{+1} 3^{+2} 5^{+1} 7^{+2}\right)$ & 2 & 8820 & $\mathrm{II}\left(4_{7}^{+1} 3^{-2} 5^{-1} 7^{+2}\right)$ & 2 & 8820 & $\mathrm{I}\left(4_{1}^{+1} 3^{+2} 5^{-1} 7^{+2}\right)$ \\
\hline 820 & $\left.-25^{-1} 7^{+2}\right)$ & 3 & 9075 & $\mathrm{I}\left(3^{+1} 5^{-2} 11^{-2}\right)$ & 2 & 9126 & $\mathrm{II}\left(2_{1}^{+1} 3^{-1} 9^{-1} 13^{+2}\right)$ \\
\hline 360 & $\mathrm{I}\left(4_{\mathrm{II}}^{-2} 3^{+2} 5^{+1} 13^{-1}\right)$ & 3 & 9408 & $\left(4_{7}^{+1} 16_{7}^{+1} 3^{+1} 7^{+2}\right)$ & 6 & 9450 & $\operatorname{II}\left(2_{7}^{+1} 3^{-1} 9^{+1} 5^{-2} 7^{-1}\right)$ \\
\hline 47 & $\mathrm{I}\left(3^{+1} 9^{-1} 19^{+2}\right)$ & 3 & 9900 & $\mathrm{I}\left(2_{\mathrm{II}}^{+2} 3^{+2} 5^{-2} 11^{+1}\right)$ & 4 & 9900 & $\left.5^{-2} 11^{-1}\right)$ \\
\hline
\end{tabular}




\begin{tabular}{|c|c|c|c|c|c|c|c|}
\hline 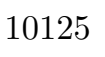 & $\mathrm{I}\left(9^{+2} 5^{+1} 25^{-1}\right)$ & 3 & 0 & $11\left(4_{5}\right.$ & 3 & 10404 & $11\left(4_{3}\right.$ \\
\hline 0500 & $\left(4_{3}^{-1} 3^{-1} 5^{+1} 25^{+1} 7^{+1}\right)$ & 3 & 0800 & $\mathrm{I}\left(4_{2}^{+2} 3^{-1} 9^{+1} 5^{-2}\right)$ & 2 & 10800 & \\
\hline 800 & $\mathrm{I}\left(4_{2}^{+2} 3^{-1} 9^{-1} 5^{-2}\right)$ & 2 & 800 & $\mathrm{I}\left(4_{6}^{+2} 3^{+1} 9^{+1} 5^{-2}\right)$ & & 800 & $\mathrm{I}\left(4_{\mathrm{II}}^{+2} 3\right.$ \\
\hline 800 & $\left.\mathrm{II}_{\mathrm{II}}^{+2} 3^{+1} 9^{-1} 5^{+2}\right)$ & 3 & 0800 & $\mathrm{I}\left(4_{\mathrm{II}}^{+2} 3^{-1} 9^{-1} 5^{-2}\right)$ & & 10800 & $\mathrm{I}\left(4_{\mathrm{II}}^{-2}\right.$ \\
\hline 800 & $\mathrm{I}\left(4_{\mathrm{II}}^{-2} 3^{-1} 9^{-1} 5^{+2}\right)$ & 1 & 0800 & $\mathrm{I}\left(4_{\mathrm{II}}^{-2} 3^{+1} 9^{+1} 5^{-2}\right)$ & 2 & 10800 & $\mathrm{II}\left(16_{1}^{+1} 3^{+1} 9^{+1} 5^{-2}\right)$ \\
\hline 890 & $\mathrm{II}\left(2_{7}^{+1} 3^{+2} 5^{+1} 11^{+2}\right)$ & 4 & 11025 & $\mathrm{I}\left(3^{-2} 5^{-2} 7^{+2}\right)$ & 2 & 11025 & \\
\hline 025 & $\left.27^{+2}\right)$ & 2 & 1025 & & & 1520 & $\mathrm{I}\left(16_{\mathrm{I}}^{-}\right.$ \\
\hline 700 & $\mathrm{II}\left(4_{7}^{+1}\right.$ & 3 & 1760 & $\mathrm{I}\left(4_{\mathrm{II}}^{-2}\right.$ & 2 & 11760 & \\
\hline 1760 & $\left.5^{-1} 7^{+2}\right)$ & 2 & 1760 & $\left.{ }^{1} 7^{+2}\right)$ & & 11760 & $\left.+17^{+2}\right)$ \\
\hline 2096 & $4_{1}^{+1} 16$ & 12 & 12348 & $\mathrm{II}\left(4_{5}^{-1}\right.$ & 2 & 12675 & $\mathrm{I}\left(3^{+}\right.$ \\
\hline 996 & & 2 & 13068 & $\mathrm{II}\left(4_{1}^{+}\right.$ & 2 & 13068 & $\mathrm{II}\left(4_{1}^{+1}\right.$ \\
\hline 068 & $\mathrm{II}\left(4_{1}^{+}\right.$ & 3 & 3200 & & 2 & 13230 & \\
\hline 3500 & $?^{+2}$ & 4 & 500 & $\mathrm{I}\left(2_{2}^{+2}\right.$ & 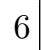 & 13500 & \\
\hline 500 & $\left.{ }^{1} 5^{+1} 25^{-1}\right)$ & 2 & 13500 & $\mathrm{II}\left(4_{5}^{-1} 3\right.$ & 2 & 13500 & $\mathrm{II}(4$ \\
\hline 500 & $\left.5^{-1} 25^{+1}\right)$ & 6 & 13500 & $\mathrm{I}\left(4_{7}^{+1} 3\right.$ & 6 & 13520 & \\
\hline 552 & $\left.11^{+2}\right)$ & 3 & 3872 & & 2 & 4700 & \\
\hline 700 & $\mathrm{I}\left(2_{\mathrm{II}}^{+2}\right.$ & 2 & 14700 & & 2 & 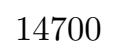 & \\
\hline 700 & $\left.5^{-2} 7^{-2}\right)$ & 2 & 1700 & $\mathrm{I}\left(4_{7}^{+}\right.$ & 3 & 14700 & \\
\hline 120 & $\left.5^{+1} 7^{+1}\right)$ & 3 & 15120 & $\mathrm{I}\left(4_{\mathrm{II}}^{-2}\right.$ & 2 & 15120 & $\left.5^{-1} 7^{+1}\right)$ \\
\hline 600 & $\left.-213^{+1}\right)$ & 3 & 15876 & & 3 & 16128 & \\
\hline 128 & & 4 & 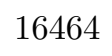 & & 2 & 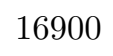 & \\
\hline 328 & $\left(9^{+2}\right)$ & 2 & 7424 & & 2 & 17424 & $\left.{ }^{2} 11^{+2}\right)$ \\
\hline 424 & $\left.11^{+2}\right)$ & 3 & 18000 & $-1)$ & 3 & 000 & $-1)$ \\
\hline 000 & 1) & 1 & 3000 & & 1 & 00 & \\
\hline 000 & & 3 & & $\mathrm{II}(2$ & 4 & & \\
\hline 252 & $\mathrm{H}^{+} \mathrm{A}^{+}$ & 2 & 5 & $\mathrm{I}(3$ & 3 & 18900 & $\mathrm{I}\left(2_{\mathrm{II}}^{+2}\right.$ \\
\hline 900 & $\left.5^{-2} 7^{-1}\right)$ & 2 & 8900 & $\left.-27^{-1}\right)$ & 2 & 18900 & $\left.{ }^{+1} 5^{+2} 7^{-1}\right)$ \\
\hline 9044 & $\left.{ }^{2} 23^{+2}\right)$ & 3 & 19200 & & 2 & 9600 & \\
\hline 600 & & 2 & 736 & & 3 & 0736 & \\
\hline & $\left.{ }^{1} 7^{+2}\right)$ & 3 & 68 & $\left.7^{-2}\right)$ & 2 & 21168 & $\left.{ }^{1} 7^{+2}\right)$ \\
\hline 168 & $\left.-17^{-2}\right)$ & 2 & 21168 & $\left.7^{+2}\right)$ & 1 & 21168 & $\left.-17^{+2}\right)$ \\
\hline 1168 & & 6 & 21600 & & 4 & 21600 & $\left.3^{-1} 9^{-1} 5^{-2}\right)$ \\
\hline 600 & $\mathrm{I}\left(2_{1}^{+1} 16_{3}^{-1} 3^{-1} 9^{-1} 5^{-2}\right)$ & 4 & 21600 & & 4 & 21780 & \\
\hline 8 & $\left.-^{-1} 11^{-2}\right)$ & 2 & 22050 & $\left.5^{-2} 7^{+2}\right)$ & 2 & 22050 & $\left.5^{-2} 7^{+2}\right)$ \\
\hline 3716 & $\left.{ }^{2} 11^{+2}\right)$ & 3 & 24336 & & 1 & 25200 & \\
\hline 5200 & & 2 & 25200 & $\left.5^{-2} 7^{-1}\right)$ & 2 & 25200 & \\
\hline 200 & $\left.5^{-2} 7^{+1}\right)$ & 3 & 25392 & & 3 & 26460 & $\left.{ }^{+1} 5^{+1} 7^{+2}\right)$ \\
\hline 460 & $\left.{ }^{-1} 5^{-1} 7^{+2}\right)$ & 3 & 26460 & & 2 & 26460 & $\left.{ }^{-1} 5^{+1} 7^{+2}\right)$ \\
\hline 7225 & $\mathrm{I}\left(3^{+2} 5^{-2} 11^{-2}\right)$ & 2 & 28224 & $\mathrm{I}\left(4_{1}^{+1} 1\right.$ & & 29040 & $3 \quad 11)$ \\
\hline
\end{tabular}




\begin{tabular}{|c|c|c|c|c|c|c|c|}
\hline 0420 & $\mathrm{II}\left(4_{7}^{+1} 3^{+2} 5^{-1} 13^{+2}\right)$ & 3 & 31500 & $\mathrm{III}\left(4_{1}^{+1} 3^{+2} 5^{-1} 25^{-1} 7^{-1}\right)$ & 3 & 32000 & $\mathrm{I}\left(16_{2}^{+2} 5^{+1} 25^{+1}\right)$ \\
\hline 32000 & $\mathrm{I}\left(16_{6}^{-2} 5^{+1} 25^{+1}\right)$ & 6 & 32400 & $\mathrm{I}\left(4_{\mathrm{II}}^{-2} 9^{+2} 5^{+2}\right)$ & 2 & 33075 & $\mathrm{I}\left(3^{-1} 9^{+1} 5^{-2} 7^{+2}\right)$ \\
\hline 33075 & $\mathrm{I}\left(3^{-1} 9^{-1} 5^{-2} 7^{+2}\right)$ & 2 & 33075 & $\mathrm{I}\left(3^{+1} 9^{+1} 5^{+2} 7^{+2}\right)$ & 3 & 34560 & $\mathrm{I}\left(16_{\mathrm{II}}^{-2} 3^{-1} 9^{-1} 5^{+1}\right)$ \\
\hline 35280 & $\mathrm{I}\left(4_{\mathrm{II}}^{+2} 3^{+2} 5^{-1} 7^{+2}\right)$ & 3 & 35280 & $\mathrm{I}\left(4_{6}^{+2} 3^{+2} 5^{-1} 7^{+2}\right)$ & 3 & 35280 & $\mathrm{I}\left(4_{\mathrm{II}}^{-2} 3^{+2} 5^{-1} 7^{-2}\right)$ \\
\hline 35280 & $\mathrm{I}\left(4_{\mathrm{II}}^{-2} 3^{+2} 5^{+1} 7^{+2}\right)$ & 2 & 35280 & $\mathrm{I}\left(4_{\mathrm{II}}^{-2} 3^{-2} 5^{-1} 7^{+2}\right)$ & 2 & 36300 & $\mathrm{I}\left(2_{\mathrm{II}}^{+2} 3^{+1} 5^{-2} 11^{+2}\right)$ \\
\hline 36300 & $\mathrm{II}\left(4_{1}^{+1} 3^{+1} 5^{-2} 11^{-2}\right)$ & 2 & 37632 & $\mathrm{I}\left(16_{6}^{+2} 3^{+1} 7^{+2}\right)$ & 4 & 37632 & $\mathrm{I}\left(16_{2}^{-2} 3^{+1} 7^{+2}\right)$ \\
\hline 38025 & $\mathrm{I}\left(3^{+2} 5^{-2} 13^{+2}\right)$ & 3 & 38988 & $\mathrm{II}\left(4_{1}^{+1} 3^{+1} 9^{-1} 19^{+2}\right)$ & 3 & 39600 & $\mathrm{I}\left(4_{\mathrm{II}}^{-2} 3^{+2} 5^{-2} 11^{-1}\right)$ \\
\hline 40500 & $\mathrm{II}\left(4_{7}^{+1} 9\right.$ & 3 & 40560 & $\mathrm{I}\left(4_{\mathrm{II}}^{-2} 3^{-1} 5^{+1} 13^{+2}\right)$ & 3 & 41616 & $\mathrm{I}\left(4_{\mathrm{II}}^{-2} 3^{+2} 17^{-2}\right)$ \\
\hline 42000 & $\mathrm{I}\left(4_{\mathrm{II}}^{-2} 3^{-1} 5^{+1} 25^{+1} 7^{+1}\right)$ & 3 & 44100 & $\mathrm{I}\left(2_{2}^{+2} 3^{+2} 5^{-2} 7^{+2}\right)$ & 3 & 44100 & $\left.5^{-2} 7^{+2}\right)$ \\
\hline 44100 & $\mathrm{II}\left(4_{3}^{-1} 3^{-2} 5^{-2} 7^{+2}\right)$ & 2 & 44100 & $\left.{ }^{+2} 5^{-2} 7^{-2}\right)$ & 2 & 44100 & $\left.5^{+2} 7^{+2}\right)$ \\
\hline 44100 & $\mathrm{II}\left(4_{7}^{+1} 3^{+2} 5^{-2} 7^{+2}\right)$ & 3 & 44100 & $\mathrm{I}\left(4_{1}^{+1} 3^{+2} 5^{-2} 7^{+2}\right)$ & 3 & 46800 & $\mathrm{I}\left(4_{\mathrm{II}}^{-2} 3^{+2} 5^{-2} 13^{+1}\right)$ \\
\hline 48384 & $\left.{ }^{+1} 7^{-1}\right)$ & 6 & 48384 & $\left.9^{+1} 7^{-1}\right)$ & 6 & 49392 & $\left.{ }^{+1} 49^{-1}\right)$ \\
\hline 50700 & $\left.{ }^{1} 5^{-2} 13^{+2}\right)$ & 3 & 51984 & $\left.+219^{+2}\right)$ & 2 & 52272 & $\left.{ }^{1} 9^{+1} 11^{-2}\right)$ \\
\hline 52272 & $\left.{ }^{1} 9^{-1} 11^{-2}\right)$ & 2 & 52272 & $\left.{ }^{1} 9^{+1} 11^{+2}\right)$ & 3 & 54000 & $\left.9^{-1} 5^{+1} 25^{+1}\right)$ \\
\hline 54000 & $\mathrm{I}\left(4_{\mathrm{II}}^{-2} 3^{-1} 9^{-1} 5^{+1} 25^{-1}\right)$ & 2 & 54000 & $\mathrm{I}\left(4_{\mathrm{II}}^{-2} 3^{+1} 9^{+1} 5^{-1} 25^{-1}\right)$ & 2 & 54000 & $\mathrm{I}\left(4_{\mathrm{II}}^{-2} 3^{+1} 9^{-1} 5^{-1} 25^{-1}\right)$ \\
\hline 54000 & $\mathrm{I}\left(4_{\mathrm{II}}^{+2} 3^{-1} 9^{+1} 5^{-1} 25^{+1}\right)$ & 6 & 54000 & $\mathrm{I}\left(4_{2}^{+2} 3^{-1} 9^{+1} 5^{-1} 25^{+1}\right)$ & 6 & 54450 & $\mathrm{II}\left(2_{7}^{+1} 3^{+2} 5^{-2} 11^{+2}\right)$ \\
\hline 55125 & $\mathrm{I}\left(3^{+2} 5^{+1} 25^{+1} 7^{+2}\right)$ & 3 & 57600 & $\mathrm{I}\left(16_{\mathrm{II}}^{-2} 3^{+2} 5^{-2}\right)$ & 2 & 58800 & $\left.5^{-2} 7^{+2}\right)$ \\
\hline 58800 & $\mathrm{I}\left(4_{\mathrm{II}}^{+2} 3^{+1} 5^{-2} 7^{+2}\right)$ & 3 & 58800 & $\mathrm{I}\left(4_{\mathrm{II}}^{-2}\right.$ & 2 & 58800 & $\mathrm{I}\left(4_{\mathrm{II}}^{-2} 3^{+1} 5^{+2} 7^{+2}\right)$ \\
\hline 58800 & $\left.{ }^{1} 5^{-2} 7^{-2}\right)$ & 2 & 66150 & $\left.9^{+1} 5^{-2} 7^{+2}\right)$ & 4 & 67600 & $\mathrm{I}\left(4_{\mathrm{II}}^{-2} 5^{-2} 13^{-2}\right)$ \\
\hline 73008 & $\left.{ }^{-1} 13^{-2}\right)$ & 2 & 73500 & $\left.5^{-1} 25^{-1} 7^{+2}\right)$ & 3 & 75600 & $\mathrm{I}\left(4_{\mathrm{II}}^{-2} 3^{-1} 9^{+1} 5^{-2} 7^{-1}\right)$ \\
\hline 75600 & $\left.9^{-1} 5^{-2} 7^{-1}\right)$ & 2 & 75600 & $\left.9^{+1} 5^{+2} 7^{-1}\right)$ & 3 & 76176 & $\left.3^{+2} 23^{+2}\right)$ \\
\hline 84672 & $\left.3^{+1} 9^{+1} 7^{+2}\right)$ & 12 & 87120 & $\mathrm{I}\left(4_{\mathrm{II}}^{-2} 3^{+2} 5^{-1} 11^{-2}\right)$ & 2 & 94864 & $\mathrm{I}\left(4_{\mathrm{II}}^{-2} 7^{+2} 11^{+2}\right)$ \\
\hline 5840 & $\left.{ }^{-1} 5^{-1} 7^{+2}\right)$ & 3 & 105840 & $\left.9^{+1} 5^{+1} 7^{+2}\right)$ & 2 & 105840 & $\left.9^{-1} 5^{+1} 7^{+2}\right)$ \\
\hline 108900 & $\mathrm{I}\left(2_{\mathrm{II}}^{+2} 3^{+2} 5^{-2} 11^{+2}\right)$ & 4 & 108900 & $\mathrm{II}\left(4_{3}^{-1} 3^{+2} 5^{-2} 11^{-2}\right)$ & 2 & 112896 & $\left.3^{+2} 7^{+2}\right)$ \\
\hline 112896 & $\mathrm{I}\left(16_{2}^{+2} 3^{+2} 7^{+2}\right)$ & 4 & 121680 & $\left.{ }^{+2} 5^{-1} 13^{+2}\right)$ & 3 & 126000 & $\left.5^{-1} 25^{-1} 7^{-1}\right)$ \\
\hline 132300 & $\left.15^{-2} 7^{+2}\right)$ & 4 & 132300 & $\left.{ }^{1} 9^{+1} 5^{-2} 7^{+2}\right)$ & 2 & 132300 & $\left.9^{-1} 5^{-2} 7^{+2}\right)$ \\
\hline 132300 & $\mathrm{II}\left(4_{1}^{+1} 3^{+1} 9^{+1} 5^{+2} 7^{+2}\right)$ & 3 & 145200 & $\mathrm{I}\left(4_{\mathrm{II}}^{-2} 3^{+1} 5^{-2} 11^{-2}\right)$ & 2 & 152100 & $\mathrm{II}\left(4_{3}^{-1} 3^{+2} 5^{-2} 13^{+2}\right)$ \\
\hline 155952 & $\mathrm{I}\left(4_{\mathrm{II}}^{-2} 3^{+1} 9^{-1} 19^{+2}\right)$ & 3 & 162000 & $\mathrm{I}\left(4_{\mathrm{II}}^{-2} 9^{+2} 5^{+1} 25^{-1}\right)$ & 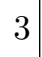 & 172800 & $\mathrm{I}\left(16_{\mathrm{II}}^{-2} 3^{+1} 9^{+1} 5^{-2}\right)$ \\
\hline 176400 & $\mathrm{I}\left(4_{\mathrm{II}}^{-2} 3^{-2} 5^{-2} 7^{+2}\right)$ & 2 & 176400 & $\mathrm{I}\left(4_{\mathrm{II}}^{-2} 3^{+2} 5^{-2} 7^{-2}\right)$ & 2 & 176400 & $\mathrm{I}\left(4_{\mathrm{II}}^{-2} 3^{+2} 5^{+2} 7^{+2}\right)$ \\
\hline 176400 & $\mathrm{I}\left(4_{6}^{+2} 3^{+2} 5^{-2} 7^{+2}\right)$ & 3 & 176400 & $\mathrm{I}\left(4_{\mathrm{II}}^{+2} 3^{+2} 5^{-2} 7^{+2}\right)$ & 3 & 202800 & $\left.5^{-2} 13^{+2}\right)$ \\
\hline 220500 & $\mathrm{II}\left(4_{7}^{+1} 3^{+2} 5^{+1} 25^{+1} 7^{+2}\right)$ & 3 & 294000 & $\mathrm{I}\left(4_{\mathrm{II}}^{-2} 3^{-1} 5^{-1} 25^{-1} 7^{+2}\right)$ & & 338688 & $\mathrm{I}\left(16_{6}^{+2} 3^{+1} 9^{+1} 7^{+2}\right)$ \\
\hline 338688 & $\left.{ }^{+1} 9^{+1} 7^{+2}\right)$ & 6 & 435600 & $\mathrm{I}\left(4_{\mathrm{II}}^{-2} 3^{+2} 5^{-2} 11^{-2}\right)$ & 2 & 529200 & $\mathrm{I}\left(4_{\mathrm{II}}^{-2} 3^{-1} 9^{+1} 5^{-2} 7^{+2}\right)$ \\
\hline 529200 & $\left.{ }^{1} 9^{-1} 5^{-2} 7^{+2}\right)$ & 2 & 529200 & $\mathrm{I}\left(4_{\mathrm{II}}^{-2} 3^{+1} 9^{+1} 5^{+2} 7^{+2}\right)$ & & 608400 & $\left.{ }^{+2} 5^{-2} 13^{+2}\right)$ \\
\hline 882000 & $\left.5^{+1} 25^{+1} 7^{+2}\right)$ & 3 & & & & & \\
\hline
\end{tabular}

TABLE 1. Totally-reflective genera in dimension 3 . 

APPENDIX C

\section{Primitive Totally-Reflective Genera of Dimension}

\section{4}

\begin{tabular}{|c|c|c|c|c|c|c|c|c|}
\hline $\operatorname{det}$ & Genus & $\mathrm{h}$ & det & Genus & $\mathrm{h}$ & $\operatorname{det}$ & Genus & $\mathrm{h}$ \\
\hline 1 & $\mathrm{I}\left(1_{4}^{+4}\right)$ & 1 & 2 & $\mathrm{I}\left(2_{1}^{+1}\right)$ & 1 & 3 & $\mathrm{I}\left(3^{-1}\right)$ & 1 \\
\hline 3 & $\mathrm{I}\left(3^{+1}\right)$ & 1 & 4 & $\mathrm{II}\left(2_{\mathrm{II}}^{-2}\right)$ & 1 & 4 & $\mathrm{I}\left(2_{2}^{+2}\right)$ & 1 \\
\hline 4 & $\mathrm{I}\left(4_{1}^{+1}\right)$ & 1 & 4 & $\mathrm{I}\left(4_{7}^{+1}\right)$ & 1 & 5 & $\mathrm{II}\left(5^{+1}\right)$ & 1 \\
\hline 5 & $\mathrm{I}\left(5^{+1}\right)$ & 1 & 5 & $\mathrm{I}\left(5^{-1}\right)$ & 1 & 6 & $\mathrm{I}\left(2_{1}^{+1} 3^{-1}\right)$ & 2 \\
\hline 6 & $\mathrm{I}\left(2_{1}^{+1} 3^{+1}\right)$ & 1 & 7 & $\mathrm{I}\left(7^{+1}\right)$ & 2 & 8 & $\mathrm{I}\left(2_{3}^{+3}\right)$ & 1 \\
\hline 8 & $\mathrm{II}\left(2_{3}^{+1} 4_{1}^{+1}\right)$ & 1 & 8 & $\mathrm{I}\left(2_{1}^{+1} 4_{1}^{+1}\right)$ & 1 & 9 & $\mathrm{II}\left(3^{+2}\right)$ & 1 \\
\hline 9 & $\mathrm{I}\left(3^{+2}\right)$ & 1 & 9 & $\mathrm{I}\left(3^{-2}\right)$ & 1 & 9 & $\mathrm{I}\left(9^{-1}\right)$ & 1 \\
\hline 10 & $\mathrm{I}\left(2_{1}^{+1} 5^{-1}\right)$ & 2 & 12 & $\mathrm{II}\left(2_{2}^{+2} 3^{-1}\right)$ & 1 & 12 & $\mathrm{II}\left(2_{6}^{+2} 3^{+1}\right)$ & 1 \\
\hline 12 & $\mathrm{I}\left(2_{2}^{+2} 3^{-1}\right)$ & 1 & 12 & $\mathrm{I}\left(2_{\mathrm{II}}^{+2} 3^{-1}\right)$ & 1 & 12 & $\mathrm{I}\left(2_{2}^{+2} 3^{+1}\right)$ & 1 \\
\hline 12 & $\mathrm{I}\left(2_{\mathrm{II}}^{+2} 3^{+1}\right)$ & 1 & 12 & $\mathrm{I}\left(4_{1}^{+1} 3^{-1}\right)$ & 1 & 12 & $\mathrm{I}\left(4_{7}^{+1} 3^{-1}\right)$ & 1 \\
\hline 12 & $\mathrm{I}\left(4_{1}^{+1} 3^{+1}\right)$ & 2 & 12 & $\mathrm{I}\left(4_{7}^{+1} 3^{+1}\right)$ & 2 & 14 & $\mathrm{I}\left(2_{1}^{+1} 7^{+1}\right)$ & 3 \\
\hline 15 & $\mathrm{I}\left(3^{+1} 5^{+1}\right)$ & 2 & 15 & $\mathrm{I}\left(3^{-1} 5^{-1}\right)$ & 3 & 15 & $\mathrm{I}\left(3^{+1} 5^{-1}\right)$ & 1 \\
\hline 16 & $\mathrm{I}\left(2_{2}^{+2} 4_{1}^{+1}\right)$ & 1 & 16 & $\operatorname{II}\left(4_{4}^{-2}\right)$ & 1 & 16 & $\mathrm{I}\left(4_{\mathrm{II}}^{-2}\right)$ & 1 \\
\hline 16 & $\mathrm{I}\left(4_{2}^{+2}\right)$ & 1 & 16 & $\mathrm{I}\left(4_{6}^{+2}\right)$ & 1 & 16 & $\mathrm{I}\left(16_{5}^{-1}\right)$ & 2 \\
\hline 16 & $\mathrm{I}\left(16_{1}^{+1}\right)$ & 2 & 18 & $\mathrm{I}\left(2_{1}^{+1} 3^{-2}\right)$ & 2 & 18 & $\mathrm{I}\left(2_{1}^{+1} 3^{+2}\right)$ & 2 \\
\hline 20 & $\mathrm{II}\left(2_{\mathrm{II}}^{+2} 5^{+1}\right)$ & 1 & 20 & $\mathrm{I}\left(2_{2}^{+2} 5^{-1}\right)$ & 2 & 20 & $\mathrm{I}\left(2_{2}^{+2} 5^{+1}\right)$ & 2 \\
\hline 20 & $\mathrm{II}\left(2_{\mathrm{II}}^{-2} 5^{-1}\right)$ & 1 & 20 & $\mathrm{I}\left(4_{7}^{+1} 5^{+1}\right)$ & 2 & 20 & $\mathrm{I}\left(4_{1}^{+1} 5^{+1}\right)$ & 2 \\
\hline 21 & $\mathrm{II}\left(3^{-1} 7^{+1}\right)$ & 1 & 21 & $\mathrm{I}\left(3^{-1} 7^{+1}\right)$ & 2 & 24 & $\mathrm{I}\left(2_{3}^{+3} 3^{+1}\right)$ & 2 \\
\hline 24 & $\mathrm{I}\left(2_{3}^{+3} 3^{-1}\right)$ & 1 & 24 & $\operatorname{II}\left(2_{1}^{+1} 4_{1}^{+1} 3^{-1}\right)$ & 2 & 24 & $\mathrm{II}\left(2_{5}^{+1} 4_{1}^{+1} 3^{+1}\right)$ & 1 \\
\hline 24 & $\mathrm{I}\left(2_{1}^{+1} 4_{1}^{+1} 3^{-1}\right)$ & 2 & 24 & $\mathrm{I}\left(2_{1}^{+1} 4_{1}^{+1} 3^{+1}\right)$ & 2 & 25 & $\mathrm{II}\left(5^{-2}\right)$ & 1 \\
\hline 25 & $\mathrm{I}\left(5^{-2}\right)$ & 1 & 25 & $\mathrm{I}\left(5^{+2}\right)$ & 2 & 27 & $\mathrm{I}\left(3^{+3}\right)$ & 1 \\
\hline 27 & $\mathrm{I}\left(3^{-1} 9^{-1}\right)$ & 2 & 27 & $\mathrm{I}\left(3^{-3}\right)$ & 1 & 27 & $\mathrm{I}\left(3^{+1} 9^{-1}\right)$ & 1 \\
\hline 27 & $\mathrm{I}\left(3^{+1} 9^{+1}\right)$ & 1 & 28 & $\mathrm{II}\left(2_{2}^{+2} 7^{+1}\right)$ & 2 & 28 & $\mathrm{I}\left(2_{\mathrm{II}}^{+2} 7^{+1}\right)$ & 2 \\
\hline 28 & $\mathrm{I}\left(4_{7}^{+1} 7^{+1}\right)$ & 3 & 30 & $\mathrm{I}\left(2_{1}^{+1} 3^{-1} 5^{-1}\right)$ & 4 & 32 & $\mathrm{I}\left(2_{1}^{+1} 4_{2}^{+2}\right)$ & 1 \\
\hline 32 & $\mathrm{I}\left(2_{1}^{+1} 4_{\mathrm{II}}^{+2}\right)$ & 1 & 32 & $\mathrm{I}\left(2_{1}^{+1} 16_{3}^{-1}\right)$ & 2 & 32 & $\mathrm{I}\left(2_{1}^{+1} 16_{5}^{-1}\right)$ & 1 \\
\hline 32 & $\mathrm{I}\left(2_{1}^{+1} 16_{1}^{+1}\right)$ & 2 & 32 & $\mathrm{I}\left(2_{1}^{+1} 16_{7}^{+1}\right)$ & 1 & 33 & $\mathrm{I}\left(3^{+1} 11^{-1}\right)$ & 2 \\
\hline 36 & $\mathrm{II}\left(2_{\mathrm{II}}^{-2} 3^{-2}\right)$ & 1 & 36 & $\mathrm{II}\left(2_{\mathrm{II}}^{-2} 9^{-1}\right)$ & 1 & 36 & $\mathrm{II}\left(2_{\mathrm{II}}^{-2} 9^{+1}\right)$ & 1 \\
\hline
\end{tabular}




\begin{tabular}{|c|c|c|c|c|c|c|c|}
\hline 36 & $\mathrm{II}\left(2_{\mathrm{II}}^{+2} 3^{+2}\right)$ & 1 & 36 & $\operatorname{II}\left(2_{0}^{+2} 3^{+2}\right)$ & 1 & 36 & $\mathrm{I}\left(2_{\mathrm{II}}^{+2} 3^{+2}\right)$ \\
\hline 36 & $\mathrm{I}\left(2_{2}^{+2} 3^{-2}\right)$ & 2 & 36 & $\mathrm{I}\left(2_{2}^{+2} 3^{+2}\right)$ & 2 & 36 & $\mathrm{I}\left(4_{7}^{+1} 3^{+2}\right)$ \\
\hline 36 & $\mathrm{I}\left(4_{1}^{+1} 3^{-2}\right)$ & 2 & 36 & $\mathrm{I}\left(4_{7}^{+1} 3^{-2}\right)$ & 2 & 36 & $\mathrm{I}\left(4_{1}^{+1} 3^{+2}\right)$ \\
\hline 40 & $\mathrm{I}\left(2_{3}^{+3} 5^{+1}\right)$ & 2 & 40 & $\mathrm{II}\left(2_{3}^{+1} 4_{1}^{+1} 5^{-1}\right)$ & 2 & 45 & $\mathrm{II}\left(3^{+2} 5^{-1}\right)$ \\
\hline 45 & $\mathrm{II}\left(3^{-2} 5^{+1}\right)$ & 1 & 45 & $\mathrm{I}\left(3^{+2} 5^{-1}\right)$ & 2 & 45 & $\mathrm{I}\left(3^{-2} 5^{+1}\right)$ \\
\hline 48 & $\mathrm{I}\left(2_{2}^{+2} 4_{1}^{+1} 3^{+1}\right)$ & 2 & 48 & $\mathrm{I}\left(2_{\mathrm{II}}^{+2} 4_{1}^{+1} 3^{+1}\right)$ & 1 & 48 & $\mathrm{I}\left(2_{2}^{+2} 4_{1}^{+1} 3^{-1}\right)$ \\
\hline 48 & $\mathrm{II}\left(4_{2}^{+2} 3^{-1}\right)$ & 1 & 48 & $\mathrm{II}\left(4_{6}^{+2} 3^{+1}\right)$ & 1 & 48 & $\mathrm{I}\left(4_{\mathrm{II}}^{+2} 3^{-1}\right)$ \\
\hline 48 & $\mathrm{I}\left(4_{0}^{+2} 3^{-1}\right)$ & 1 & 48 & $\mathrm{I}\left(4_{2}^{+2} 3^{+1}\right)$ & 2 & 48 & $\mathrm{I}\left(4_{6}^{+2} 3^{-1}\right)$ \\
\hline 48 & $\mathrm{I}\left(4_{\mathrm{II}}^{+2} 3^{+1}\right)$ & 1 & 48 & $\mathrm{I}\left(4_{0}^{+2} 3^{+1}\right)$ & 2 & 48 & $\operatorname{II}\left(4_{2}^{-2} 3^{-1}\right)$ \\
\hline 48 & $\operatorname{II}\left(4_{6}^{-2} 3^{+1}\right)$ & 1 & 48 & $\mathrm{I}\left(4_{\mathrm{II}}^{-2} 3^{-1}\right)$ & 1 & 48 & $\mathrm{I}\left(4_{\mathrm{II}}^{-2} 3^{+1}\right)$ \\
\hline 48 & $\mathrm{I}\left(16_{1}^{+1} 3^{-1}\right)$ & 2 & 48 & $\mathrm{I}\left(16_{5}^{-1} 3^{-1}\right)$ & 2 & 49 & $\mathrm{II}\left(7^{+2}\right)$ \\
\hline 54 & $\mathrm{I}\left(2_{1}^{+1} 3^{+3}\right)$ & 2 & 54 & $\mathrm{I}\left(2_{1}^{+1} 3^{-3}\right)$ & 1 & 54 & $\mathrm{I}\left(2_{1}^{+1} 3^{+1} 9^{-1}\right)$ \\
\hline 56 & $\mathrm{I}\left(2_{3}^{+3} 7^{+1}\right)$ & 3 & 56 & $\mathrm{II}\left(2_{1}^{+1} 4_{1}^{+1} 7^{+1}\right)$ & 3 & 60 & $\mathrm{II}\left(2_{2}^{+2} 3^{+1} 5^{+1}\right)$ \\
\hline 60 & $\mathrm{II}\left(2_{2}^{+2} 3^{-1} 5^{-1}\right)$ & 3 & 60 & $\mathrm{II}\left(2_{6}^{+2} 3^{+1} 5^{-1}\right)$ & 1 & 60 & $\mathrm{I}\left(2_{\mathrm{II}}^{+2} 3^{+1} 5^{+1}\right)$ \\
\hline 60 & $\mathrm{I}\left(2_{\mathrm{II}}^{+2} 3^{-1} 5^{-1}\right)$ & 2 & 60 & $\mathrm{I}\left(2_{\mathrm{II}}^{+2} 3^{-1} 5^{+1}\right)$ & 1 & 60 & $\mathrm{I}\left(4_{7}^{+1} 3^{+1} 5^{+1}\right)$ \\
\hline 60 & $\mathrm{I}\left(4_{1}^{+1} 3^{+1} 5^{-1}\right)$ & 2 & 63 & $\mathrm{I}\left(3^{+2} 7^{-1}\right)$ & 3 & 63 & $\mathrm{I}\left(3^{+2} 7^{+1}\right)$ \\
\hline 64 & $\mathrm{I}\left(2_{2}^{+2} 16_{5}^{-1}\right)$ & 2 & 64 & $\mathrm{I}\left(2_{2}^{+2} 16_{3}^{-1}\right)$ & 1 & 64 & $\mathrm{I}\left(2_{2}^{+2} 16_{1}^{+1}\right)$ \\
\hline 64 & $\mathrm{I}\left(4_{3}^{+3}\right)$ & 1 & 64 & $\mathrm{I}\left(2_{2}^{+2} 16_{7}^{+1}\right)$ & 1 & 64 & $\mathrm{I}\left(4_{5}^{+3}\right)$ \\
\hline 64 & $\operatorname{II}\left(4_{3}^{-1} 16_{1}^{+1}\right)$ & 2 & 64 & $\mathrm{I}\left(4_{1}^{+1} 16_{1}^{+1}\right)$ & 3 & 68 & $\mathrm{II}\left(2_{\mathrm{II}}^{-2} 17^{+1}\right)$ \\
\hline 72 & $\mathrm{I}\left(2_{3}^{+3} 3^{-2}\right)$ & 2 & 72 & $\mathrm{I}\left(2_{3}^{+3} 3^{+2}\right)$ & 2 & 72 & $\mathrm{II}\left(2_{3}^{+1} 4_{1}^{+1} 3^{-2}\right)$ \\
\hline 72 & $\mathrm{II}\left(2_{7}^{+1} 4_{1}^{+1} 3^{+2}\right)$ & 2 & 72 & $\mathrm{I}\left(2_{1}^{+1} 4_{1}^{+1} 3^{-2}\right)$ & 3 & 72 & $\mathrm{I}\left(2_{1}^{+1} 4_{1}^{+1} 3^{+2}\right)$ \\
\hline 75 & $\mathrm{I}\left(3^{-1} 5^{+2}\right)$ & 3 & 75 & $\mathrm{I}\left(3^{+1} 5^{-2}\right)$ & 3 & 80 & $\mathrm{I}\left(2_{2}^{+2} 4_{1}^{+1} 5^{+1}\right)$ \\
\hline 80 & $\mathrm{II}\left(4_{0}^{+2} 5^{+1}\right)$ & 2 & 80 & $\mathrm{I}\left(4_{2}^{+2} 5^{-1}\right)$ & 2 & 80 & $\mathrm{I}\left(4_{6}^{+2} 5^{+1}\right)$ \\
\hline 80 & $\mathrm{I}\left(4_{2}^{+2} 5^{+1}\right)$ & 2 & 80 & $\mathrm{I}\left(4_{6}^{+2} 5^{-1}\right)$ & 2 & 80 & $\mathrm{I}\left(4_{\mathrm{II}}^{-2} 5^{+1}\right)$ \\
\hline 80 & $\mathrm{I}\left(16_{1}^{+1} 5^{+1}\right)$ & 5 & 80 & $\mathrm{I}\left(16_{5}^{-1} 5^{+1}\right)$ & 5 & 81 & $\mathrm{II}\left(3^{+2} 9^{-1}\right)$ \\
\hline 81 & $\mathrm{II}\left(3^{+2} 9^{+1}\right)$ & 1 & 81 & $\mathrm{I}\left(3^{+2} 9^{-1}\right)$ & 1 & 81 & $\mathrm{I}\left(3^{+2} 9^{+1}\right)$ \\
\hline 81 & $\mathrm{I}\left(3^{-2} 9^{+1}\right)$ & 1 & 81 & $\mathrm{I}\left(3^{-2} 9^{-1}\right)$ & 1 & 81 & $\mathrm{I}\left(9^{+2}\right)$ \\
\hline 84 & $\operatorname{II}\left(2_{\mathrm{II}}^{+2} 3^{-1} 7^{+1}\right)$ & 2 & 84 & $\mathrm{II}\left(2_{\mathrm{II}}^{-2} 3^{+1} 7^{+1}\right)$ & 2 & 90 & $\mathrm{I}\left(2_{1}^{+1} 3^{+2} 5^{+1}\right)$ \\
\hline 90 & $\mathrm{I}\left(2_{1}^{+1} 3^{+2} 5^{-1}\right)$ & 4 & 96 & $\mathrm{I}\left(2_{1}^{+1} 4_{\mathrm{II}}^{+2} 3^{-1}\right)$ & 2 & 96 & $\mathrm{I}\left(2_{1}^{+1} 4_{2}^{+2} 3^{+1}\right)$ \\
\hline 96 & $\mathrm{I}\left(2_{1}^{+1} 4_{\mathrm{II}}^{+2} 3^{+1}\right)$ & 1 & 96 & $\mathrm{I}\left(2_{1}^{+1} 4_{2}^{+2} 3^{-1}\right)$ & 2 & 96 & $\mathrm{I}\left(2_{1}^{+1} 16_{1}^{+1} 3^{+1}\right)$ \\
\hline 96 & $\mathrm{I}\left(2_{1}^{+1} 16_{7}^{+1} 3^{+1}\right)$ & 3 & 96 & $\mathrm{I}\left(2_{1}^{+1} 16_{5}^{-1} 3^{+1}\right)$ & 4 & 96 & $\mathrm{I}\left(2_{1}^{+1} 16_{3}^{-1} 3^{+1}\right)$ \\
\hline 98 & $\mathrm{I}\left(2_{1}^{+1} 7^{+2}\right)$ & 4 & 100 & $\mathrm{II}\left(2_{\mathrm{II}}^{-2} 5^{+2}\right)$ & 1 & 100 & $\mathrm{II}\left(2_{\mathrm{II}}^{+2} 5^{-2}\right)$ \\
\hline 100 & $\mathrm{I}\left(2_{2}^{+2} 5^{-2}\right)$ & 2 & 108 & $\mathrm{II}\left(2_{2}^{+2} 3^{+3}\right)$ & 1 & 108 & $\operatorname{II}\left(2_{2}^{+2} 3^{-1} 9^{-1}\right)$ \\
\hline 108 & $\operatorname{II}\left(2_{6}^{+2} 3^{-3}\right)$ & 1 & 108 & $\mathrm{II}\left(2_{6}^{+2} 3^{+1} 9^{-1}\right)$ & 1 & 108 & $\mathrm{II}\left(2_{6}^{+2} 3^{+1} 9^{+1}\right)$ \\
\hline 108 & $\mathrm{I}\left(2_{2}^{+2} 3^{+3}\right)$ & 1 & 108 & $\mathrm{I}\left(2_{2}^{+2} 3^{-1} 9^{-1}\right)$ & 2 & 108 & $\mathrm{I}\left(2_{\mathrm{II}}^{+2} 3^{+3}\right)$ \\
\hline 108 & $\mathrm{I}\left(2_{\mathrm{II}}^{+2} 3^{-1} 9^{+1}\right)$ & 1 & 108 & $\mathrm{I}\left(2_{\mathrm{II}}^{+2} 3^{-1} 9^{-1}\right)$ & 1 & 108 & $\mathrm{I}\left(2_{2}^{+2} 3^{-3}\right)$ \\
\hline 108 & $\mathrm{I}\left(2_{2}^{+2} 3^{+1} 9^{+1}\right)$ & 2 & 108 & $\mathrm{I}\left(2_{\mathrm{II}}^{+2} 3^{-3}\right)$ & 1 & 108 & $\mathrm{I}\left(2_{\mathrm{II}}^{+2} 3^{+1} 9^{+1}\right)$ \\
\hline 108 & $\mathrm{I}\left(4_{1}^{+1} 3^{+3}\right)$ & 2 & 108 & $\mathrm{I}\left(4_{7}^{+1} 3^{+3}\right)$ & 2 & 108 & $\mathrm{I}\left(4_{1}^{+1} 3^{-3}\right)$ \\
\hline 108 & $\mathrm{I}\left(4_{1}^{+1} 3^{+1} 9^{+1}\right)$ & 4 & 108 & $\mathrm{I}\left(4_{7}^{+1} 3^{-3}\right)$ & 1 & 108 & $\mathrm{I}\left(4_{7}^{+1} 3^{+1} 9^{-1}\right)$ \\
\hline
\end{tabular}


C. PRIMitiVE TOTALLY-REFLECTIVE GENERA OF DIMENSION 4

79

\begin{tabular}{|c|c|c|c|c|c|c|c|}
\hline 108 & $\mathrm{I}\left(4_{7}^{+1} 3^{+1} 9^{+1}\right)$ & 2 & 112 & $\mathrm{II}\left(4_{2}^{-2} 7^{+1}\right)$ & 2 & 112 & $\mathrm{I}\left(4_{\mathrm{II}}^{-2} 7^{+1}\right)$ \\
\hline 117 & $\operatorname{II}\left(3^{+2} 13^{-1}\right)$ & 2 & 120 & $\mathrm{I}\left(2_{3}^{+3} 3^{+1} 5^{+1}\right)$ & 4 & 120 & $\operatorname{II}\left(2_{1}^{+1} 4_{1}^{+1} 3^{-1} 5^{-1}\right)$ \\
\hline 125 & $\mathrm{II}\left(5^{+3}\right)$ & 1 & 125 & $\mathrm{I}\left(5^{+3}\right)$ & 1 & 125 & $\mathrm{I}\left(5^{-3}\right)$ \\
\hline 128 & $\mathrm{I}\left(2_{1}^{+1} 4_{1}^{+1} 16_{1}^{+1}\right)$ & 2 & 128 & $\mathrm{I}\left(2_{1}^{+1} 4_{1}^{+1} 16_{7}^{+1}\right)$ & 2 & 132 & $\mathrm{II}\left(2_{\mathrm{II}}^{-2} 3^{+1} 11^{-1}\right)$ \\
\hline 132 & $\mathrm{II}\left(2_{\mathrm{II}}^{-2} 3^{-1} 11^{+1}\right)$ & 2 & 135 & $\mathrm{I}\left(3^{-3} 5^{+1}\right)$ & 1 & 135 & $\mathrm{I}\left(3^{+1} 9^{-1} 5^{+1}\right)$ \\
\hline 135 & $\mathrm{I}\left(3^{+3} 5^{+1}\right)$ & 3 & 135 & $\mathrm{I}\left(3^{-3} 5^{-1}\right)$ & 2 & 144 & $\mathrm{I}\left(2_{\mathrm{II}}^{+2} 4_{7}^{+1} 3^{+2}\right)$ \\
\hline 144 & $\mathrm{I}\left(2_{2}^{+2} 4_{1}^{+1} 3^{+2}\right)$ & 2 & 144 & $\mathrm{I}\left(2_{\mathrm{II}}^{+2} 4_{1}^{+1} 3^{+2}\right)$ & 1 & 144 & $\mathrm{II}\left(4_{\mathrm{II}}^{-2} 3^{+2}\right)$ \\
\hline 144 & $\mathrm{II}\left(4_{4}^{-2} 3^{-2}\right)$ & 2 & 144 & $\mathrm{I}\left(4_{\mathrm{II}}^{-2} 3^{-2}\right)$ & 2 & 144 & $\mathrm{II}\left(4_{\mathrm{II}}^{+2} 3^{+2}\right)$ \\
\hline 144 & $\mathrm{II}\left(4_{0}^{+2} 3^{+2}\right)$ & 2 & 144 & $\mathrm{I}\left(4_{\mathrm{II}}^{+2} 3^{+2}\right)$ & 2 & 144 & $\mathrm{I}\left(4_{0}^{+2} 3^{+2}\right)$ \\
\hline 144 & $\mathrm{I}\left(4_{2}^{+2} 3^{-2}\right)$ & 2 & 144 & $\mathrm{I}\left(4_{6}^{+2} 3^{+2}\right)$ & 2 & 144 & $\mathrm{I}\left(4_{2}^{+2} 3^{+2}\right)$ \\
\hline 144 & $\mathrm{I}\left(4_{6}^{+2} 3^{-2}\right)$ & 2 & 160 & $\mathrm{I}\left(2_{1}^{+1} 4_{\mathrm{II}}^{+2} 5^{-1}\right)$ & 2 & 162 & $\mathrm{I}\left(2_{1}^{+1} 3^{-2} 9^{-1}\right)$ \\
\hline 162 & $\mathrm{I}\left(2_{1}^{+1} 3^{+2} 9^{+1}\right)$ & 2 & 162 & $\mathrm{I}\left(2_{1}^{+1} 3^{+2} 9^{-1}\right)$ & 2 & 180 & $\mathrm{II}\left(2_{\mathrm{II}}^{+2} 3^{+2} 5^{-1}\right)$ \\
\hline 180 & $\mathrm{II}\left(2_{\mathrm{II}}^{+2} 3^{-2} 5^{+1}\right)$ & 2 & 180 & $\mathrm{II}\left(2_{\mathrm{II}}^{-2} 3^{+2} 5^{+1}\right)$ & 2 & 180 & $\mathrm{II}\left(2_{\mathrm{II}}^{-2} 3^{-2} 5^{-1}\right)$ \\
\hline 189 & $\operatorname{II}\left(3^{-3} 7^{-1}\right)$ & 1 & 189 & $\mathrm{II}\left(3^{-1} 9^{-1} 7^{+1}\right)$ & 3 & 189 & $\mathrm{I}\left(3^{-3} 7^{-1}\right)$ \\
\hline 192 & $\mathrm{I}\left(2_{2}^{+2} 16_{7}^{+1} 3^{-1}\right)$ & 2 & 192 & $\mathrm{I}\left(2_{2}^{+2} 16_{1}^{+1} 3^{+1}\right)$ & 3 & 192 & $\mathrm{I}\left(4_{3}^{+3} 3^{+1}\right)$ \\
\hline 192 & $\mathrm{I}\left(4_{7}^{+3} 3^{-1}\right)$ & 1 & 192 & $\mathrm{I}\left(2_{\mathrm{II}}^{+2} 16_{1}^{+1} 3^{+1}\right)$ & 2 & 192 & $\mathrm{I}\left(2_{2}^{+2} 16_{7}^{+1} 3^{+1}\right)$ \\
\hline 192 & $\mathrm{I}\left(4_{1}^{+3} 3^{+1}\right)$ & 2 & 192 & $\mathrm{I}\left(4_{5}^{+3} 3^{-1}\right)$ & 1 & 192 & $\mathrm{I}\left(2_{2}^{+2} 16_{1}^{+1} 3^{-1}\right)$ \\
\hline 192 & $\mathrm{I}\left(2_{\mathrm{II}}^{+2} 16_{5}^{-1} 3^{+1}\right)$ & 2 & 192 & $\mathrm{I}\left(2_{2}^{+2} 16_{3}^{-1} 3^{+1}\right)$ & 3 & 192 & $\mathrm{I}\left(2_{2}^{+2} 16_{5}^{-1} 3^{-1}\right)$ \\
\hline 192 & $\mathrm{I}\left(2_{2}^{+2} 16_{3}^{-1} 3^{-1}\right)$ & 2 & 192 & $\mathrm{I}\left(2_{2}^{+2} 16_{5}^{-1} 3^{+1}\right)$ & 3 & 192 & $\operatorname{II}\left(4_{1}^{+1} 16_{1}^{+1} 3^{-1}\right)$ \\
\hline 196 & $\mathrm{II}\left(2_{\mathrm{II}}^{-2} 7^{-2}\right)$ & 2 & 216 & $\mathrm{I}\left(2_{3}^{+3} 3^{-3}\right)$ & 2 & 216 & $\mathrm{I}\left(2_{3}^{+3} 3^{+3}\right)$ \\
\hline 216 & $\mathrm{I}\left(2_{3}^{+3} 3^{-1} 9^{+1}\right)$ & 2 & 216 & $\mathrm{II}\left(2_{1}^{+1} 4_{1}^{+1} 3^{+3}\right)$ & 2 & 216 & $\mathrm{II}\left(2_{5}^{+1} 4_{1}^{+1} 3^{-3}\right)$ \\
\hline 216 & $\operatorname{II}\left(2_{5}^{+1} 4_{1}^{+1} 3^{+1} 9^{-1}\right)$ & 2 & 216 & $\mathrm{I}\left(2_{1}^{+1} 4_{1}^{+1} 3^{+3}\right)$ & 2 & 216 & $\mathrm{I}\left(2_{1}^{+1} 4_{1}^{+1} 3^{-3}\right)$ \\
\hline 224 & $\mathrm{I}\left(2_{1}^{+1} 4_{\mathrm{II}}^{+2} 7^{+1}\right)$ & 3 & 225 & $\mathrm{II}\left(3^{+2} 5^{+2}\right)$ & 2 & 225 & $\mathrm{I}\left(3^{+2} 5^{-2}\right)$ \\
\hline 240 & $\mathrm{II}\left(4_{2}^{-2} 3^{+1} 5^{+1}\right)$ & 2 & 240 & $\mathrm{II}\left(4_{2}^{-2} 3^{-1} 5^{-1}\right)$ & 3 & 240 & $\operatorname{II}\left(4_{6}^{-2} 3^{+1} 5^{-1}\right)$ \\
\hline 240 & $\mathrm{I}\left(4_{\mathrm{II}}^{-2} 3^{+1} 5^{+1}\right)$ & 2 & 240 & $\mathrm{I}\left(4_{\mathrm{II}}^{-2} 3^{-1} 5^{-1}\right)$ & 3 & 240 & $\mathrm{I}\left(4_{\mathrm{II}}^{-2} 3^{+1} 5^{-1}\right)$ \\
\hline 243 & $\mathrm{I}\left(3^{-1} 9^{+2}\right)$ & 2 & 243 & $\mathrm{I}\left(3^{+1} 9^{-2}\right)$ & 1 & 243 & $\mathrm{I}\left(3^{+1} 9^{+2}\right)$ \\
\hline 250 & $\mathrm{I}\left(2_{1}^{+1} 5^{+3}\right)$ & 2 & 252 & $\mathrm{II}\left(2_{2}^{+2} 3^{+2} 7^{-1}\right)$ & 3 & 252 & $\operatorname{II}\left(2_{6}^{+2} 3^{+2} 7^{+1}\right)$ \\
\hline 252 & $\mathrm{I}\left(2_{\mathrm{II}}^{+2} 3^{+2} 7^{-1}\right)$ & 3 & 252 & $\mathrm{I}\left(2_{\mathrm{II}}^{+2} 3^{+2} 7^{+1}\right)$ & 3 & 256 & $\mathrm{I}\left(4_{\mathrm{II}}^{-2} 16_{1}^{+1}\right)$ \\
\hline 256 & $\mathrm{I}\left(4_{\mathrm{II}}^{-2} 16_{7}^{+1}\right)$ & 1 & 256 & $\mathrm{I}\left(4_{\mathrm{II}}^{-2} 16_{3}^{-1}\right)$ & 1 & 256 & $\mathrm{I}\left(4_{2}^{+2} 16_{1}^{+1}\right)$ \\
\hline 256 & $\mathrm{I}\left(4_{\mathrm{II}}^{-2} 16_{5}^{-1}\right)$ & 1 & 256 & $\mathrm{I}\left(4_{6}^{+2} 16_{7}^{+1}\right)$ & 1 & 256 & $\mathrm{I}\left(16_{6}^{-2}\right)$ \\
\hline 256 & $\mathrm{I}\left(16_{2}^{+2}\right)$ & 5 & 270 & $\mathrm{I}\left(2_{1}^{+1} 3^{-3} 5^{+1}\right)$ & 4 & 288 & $\mathrm{I}\left(2_{1}^{+1} 4_{2}^{+2} 3^{-2}\right)$ \\
\hline 288 & $\mathrm{I}\left(2_{1}^{+1} 4_{\mathrm{II}}^{+2} 3^{-2}\right)$ & 2 & 288 & $\mathrm{I}\left(2_{1}^{+1} 4_{2}^{+2} 3^{+2}\right)$ & 3 & 288 & $\mathrm{I}\left(2_{1}^{+1} 4_{\mathrm{II}}^{+2} 3^{+2}\right)$ \\
\hline 297 & $\mathrm{I}\left(3^{-3} 11^{-1}\right)$ & 2 & 300 & $\mathrm{II}\left(2_{2}^{+2} 3^{-1} 5^{+2}\right)$ & 3 & 300 & $\mathrm{II}\left(2_{2}^{+2} 3^{+1} 5^{-2}\right)$ \\
\hline 300 & $\mathrm{I}\left(2_{\mathrm{II}}^{+2} 3^{-1} 5^{-2}\right)$ & 3 & 300 & $\mathrm{I}\left(2_{\mathrm{II}}^{+2} 3^{+1} 5^{+2}\right)$ & 3 & 320 & $\mathrm{I}\left(4_{5}^{+3} 5^{+1}\right)$ \\
\hline 320 & $\mathrm{I}\left(2_{2}^{+2} 16_{1}^{+1} 5^{+1}\right)$ & 6 & 320 & $\mathrm{I}\left(4_{3}^{+3} 5^{+1}\right)$ & 2 & 320 & $\mathrm{I}\left(2_{2}^{+2} 16_{5}^{-1} 5^{+1}\right)$ \\
\hline 320 & $\operatorname{II}\left(4_{7}^{+1} 16_{1}^{+1} 5^{+1}\right)$ & 5 & 320 & $\mathrm{I}\left(4_{1}^{+1} 16_{1}^{+1} 5^{+1}\right)$ & 10 & 324 & $\mathrm{II}\left(2_{\mathrm{II}}^{-2} 3^{-2} 9^{+1}\right)$ \\
\hline 324 & $\mathrm{II}\left(2_{\mathrm{II}}^{-2} 3^{-2} 9^{-1}\right)$ & 1 & 324 & $\mathrm{II}\left(2_{\mathrm{II}}^{-2} 9^{-2}\right)$ & 1 & 324 & $\mathrm{II}\left(2_{\mathrm{II}}^{-2} 9^{+2}\right)$ \\
\hline 324 & $\mathrm{II}\left(2_{\mathrm{II}}^{+2} 3^{+2} 9^{-1}\right)$ & 1 & 324 & $\mathrm{II}\left(2_{\mathrm{II}}^{+2} 3^{+2} 9^{+1}\right)$ & 1 & 324 & $\mathrm{II}\left(2_{0}^{+2} 3^{+2} 9^{-1}\right)$ \\
\hline 324 & $\mathrm{I}\left(2_{\mathrm{II}}^{+2} 3^{+2} 9^{+1}\right)$ & 2 & 324 & $\mathrm{I}\left(2_{2}^{+2} 3^{-2} 9^{+1}\right)$ & 2 & 324 & $\mathrm{I}\left(2_{2}^{+2} 3^{-2} 9^{-1}\right)$ \\
\hline
\end{tabular}




\begin{tabular}{|c|c|c|c|c|c|c|c|}
\hline 324 & $\mathrm{I}\left(2_{2}^{+2} 3^{+2} 9^{-1}\right)$ & 2 & 324 & $\mathrm{I}\left(2_{2}^{+2} 3^{+2} 9^{+1}\right)$ & 2 & 324 & $\mathrm{I}\left(4_{7}^{+1} 3^{+2} 9^{+1}\right)$ \\
\hline 324 & $\mathrm{I}\left(4_{1}^{+1} 3^{-2} 9^{+1}\right)$ & 2 & 324 & $\mathrm{I}\left(4_{1}^{+1} 3^{-2} 9^{-1}\right)$ & 2 & 324 & $\mathrm{I}\left(4_{7}^{+1} 3^{-2} 9^{+1}\right)$ \\
\hline 324 & $\mathrm{I}\left(4_{7}^{+1} 3^{-2} 9^{-1}\right)$ & 2 & 324 & $\mathrm{I}\left(4_{1}^{+1} 3^{+2} 9^{+1}\right)$ & 3 & 343 & $\mathrm{I}\left(7^{+3}\right)$ \\
\hline 360 & $\mathrm{I}\left(2_{3}^{+3} 3^{+2} 5^{+1}\right)$ & 4 & 360 & $\mathrm{I}\left(2_{3}^{+3} 3^{+2} 5^{-1}\right)$ & 4 & 360 & $\mathrm{II}\left(2_{3}^{+1} 4_{1}^{+1} 3^{+2} 5^{+1}\right)$ \\
\hline 360 & $\operatorname{II}\left(2_{7}^{+1} 4_{1}^{+1} 3^{+2} 5^{-1}\right)$ & 4 & 375 & $\mathrm{I}\left(3^{+1} 5^{+3}\right)$ & 3 & 375 & $\mathrm{I}\left(3^{-1} 5^{-3}\right)$ \\
\hline 375 & $\mathrm{I}\left(3^{-1} 5^{+3}\right)$ & 1 & 384 & $\mathrm{I}\left(2_{1}^{+1} 4_{1}^{+1} 16_{1}^{+1} 3^{+1}\right)$ & 6 & 384 & $\mathrm{I}\left(2_{1}^{+1} 4_{1}^{+1} 16_{7}^{+1} 3^{+1}\right)$ \\
\hline 392 & $\mathrm{I}\left(2_{3}^{+3} 7^{+2}\right)$ & 4 & 392 & $\mathrm{II}\left(2_{7}^{+1} 4_{1}^{+1} 7^{+2}\right)$ & 4 & 400 & $\mathrm{II}\left(4_{\mathrm{II}}^{-2} 5^{-2}\right)$ \\
\hline 400 & $\mathrm{I}\left(4_{6}^{+2} 5^{-2}\right)$ & 2 & 400 & $\mathrm{I}\left(4_{2}^{+2} 5^{-2}\right)$ & 2 & 405 & $\operatorname{II}\left(3^{-2} 9^{-1} 5^{+1}\right)$ \\
\hline 405 & $\operatorname{II}\left(3^{+2} 9^{+1} 5^{-1}\right)$ & 1 & 405 & $\mathrm{II}\left(3^{+2} 9^{-1} 5^{-1}\right)$ & 1 & 405 & $\mathrm{I}\left(3^{-2} 9^{-1} 5^{+1}\right)$ \\
\hline 405 & $\mathrm{I}\left(3^{+2} 9^{+1} 5^{-1}\right)$ & 2 & 405 & $\mathrm{I}\left(3^{+2} 9^{-1} 5^{-1}\right)$ & 2 & 420 & $\mathrm{II}\left(2_{\mathrm{II}}^{-2} 3^{+1} 5^{-1} 7^{+1}\right)$ \\
\hline 420 & $\operatorname{II}\left(2_{\mathrm{II}}^{-2} 3^{-1} 5^{+1} 7^{+1}\right)$ & 3 & 432 & $\mathrm{I}\left(2_{\mathrm{II}}^{+2} 4_{7}^{+1} 3^{+3}\right)$ & 1 & 432 & $\mathrm{I}\left(2_{2}^{+2} 4_{1}^{+1} 3^{-3}\right)$ \\
\hline 432 & $\mathrm{I}\left(2_{2}^{+2} 4_{1}^{+1} 3^{+1} 9^{+1}\right)$ & 4 & 432 & $\mathrm{I}\left(2_{\mathrm{II}}^{+2} 4_{1}^{+1} 3^{+1} 9^{+1}\right)$ & 3 & 432 & $\mathrm{I}\left(2_{2}^{+2} 4_{1}^{+1} 3^{+3}\right)$ \\
\hline 432 & $\mathrm{II}\left(4_{2}^{+2} 3^{+3}\right)$ & 1 & 432 & $\mathrm{II}\left(4_{2}^{+2} 3^{-1} 9^{-1}\right)$ & 2 & 432 & $\operatorname{II}\left(4_{6}^{+2} 3^{-3}\right)$ \\
\hline 432 & $\mathrm{II}\left(4_{6}^{+2} 3^{+1} 9^{-1}\right)$ & 1 & 432 & $\mathrm{II}\left(4_{6}^{+2} 3^{+1} 9^{+1}\right)$ & 1 & 432 & $\mathrm{I}\left(4_{\mathrm{II}}^{+2} 3^{+3}\right)$ \\
\hline 432 & $\mathrm{I}\left(4_{\mathrm{II}}^{+2} 3^{-1} 9^{-1}\right)$ & 2 & 432 & $\mathrm{I}\left(4_{0}^{+2} 3^{+3}\right)$ & 2 & 432 & $\mathrm{I}\left(4_{2}^{+2} 3^{-3}\right)$ \\
\hline 432 & $\mathrm{I}\left(4_{2}^{+2} 3^{+1} 9^{+1}\right)$ & 4 & 432 & $\mathrm{I}\left(4_{6}^{+2} 3^{+3}\right)$ & 2 & 432 & $\mathrm{I}\left(4_{\mathrm{II}}^{+2} 3^{-3}\right)$ \\
\hline 432 & $\mathrm{I}\left(4_{\mathrm{II}}^{+2} 3^{+1} 9^{+1}\right)$ & 2 & 432 & $\mathrm{I}\left(4_{0}^{+2} 3^{-3}\right)$ & 1 & 432 & $\mathrm{I}\left(4_{0}^{+2} 3^{+1} 9^{+1}\right)$ \\
\hline 432 & $\mathrm{II}\left(4_{2}^{-2} 3^{+3}\right)$ & 1 & 432 & $\operatorname{II}\left(4_{2}^{-2} 3^{-1} 9^{-1}\right)$ & 2 & 432 & $\operatorname{II}\left(4_{6}^{-2} 3^{-3}\right)$ \\
\hline 432 & $\mathrm{II}\left(4_{6}^{-2} 3^{+1} 9^{+1}\right)$ & 2 & 432 & $\mathrm{I}\left(4_{\mathrm{II}}^{-2} 3^{+3}\right)$ & 1 & 432 & $\mathrm{I}\left(4_{\mathrm{II}}^{-2} 3^{-1} 9^{-1}\right)$ \\
\hline 432 & $\mathrm{I}\left(4_{\mathrm{II}}^{-2} 3^{-3}\right)$ & 1 & 432 & $\mathrm{I}\left(4_{\mathrm{II}}^{-2} 3^{+1} 9^{-1}\right)$ & 1 & 432 & $\mathrm{I}\left(4_{\mathrm{II}}^{-2} 3^{+1} 9^{+1}\right)$ \\
\hline 432 & $\mathrm{I}\left(16_{7}^{+1} 3^{-3}\right)$ & 2 & 432 & $\mathrm{I}\left(16_{3}^{-1} 3^{-3}\right)$ & 2 & 441 & $\mathrm{I}\left(3^{+2} 7^{+2}\right)$ \\
\hline 448 & $\mathrm{I}\left(4_{3}^{+3} 7^{+1}\right)$ & 3 & 480 & $\mathrm{I}\left(2_{1}^{+1} 4_{\mathrm{II}}^{+2} 3^{-1} 5^{-1}\right)$ & 4 & 484 & $\mathrm{II}\left(2_{\mathrm{II}}^{-2} 11^{-2}\right)$ \\
\hline 486 & $\mathrm{I}\left(2_{1}^{+1} 3^{+1} 9^{+2}\right)$ & 2 & 500 & $\mathrm{II}\left(2_{\mathrm{II}}^{+2} 5^{+3}\right)$ & 1 & 500 & $\mathrm{I}\left(2_{2}^{+2} 5^{-3}\right)$ \\
\hline 500 & $\mathrm{I}\left(2_{2}^{+2} 5^{+3}\right)$ & 2 & 500 & $\mathrm{II}\left(2_{\mathrm{II}}^{-2} 5^{-3}\right)$ & 1 & 500 & $\mathrm{I}\left(4_{7}^{+1} 5^{+3}\right)$ \\
\hline 500 & $\mathrm{I}\left(4_{1}^{+1} 5^{+3}\right)$ & 2 & 512 & $\mathrm{I}\left(2_{1}^{+1} 16_{2}^{-2}\right)$ & 2 & 512 & $\mathrm{I}\left(2_{1}^{+1} 16_{6}^{-2}\right)$ \\
\hline 512 & $\mathrm{I}\left(2_{1}^{+1} 16_{4}^{-2}\right)$ & 2 & 512 & $\mathrm{I}\left(2_{1}^{+1} 16_{2}^{+2}\right)$ & 2 & 512 & $\mathrm{I}\left(2_{1}^{+1} 16_{0}^{+2}\right)$ \\
\hline 512 & $\mathrm{I}\left(2_{1}^{+1} 16_{6}^{+2}\right)$ & 1 & 540 & $\mathrm{II}\left(2_{2}^{+2} 3^{-3} 5^{+1}\right)$ & 1 & 540 & $\mathrm{II}\left(2_{2}^{+2} 3^{+1} 9^{-1} 5^{+1}\right)$ \\
\hline 540 & $\mathrm{II}\left(2_{6}^{+2} 3^{+3} 5^{+1}\right)$ & 3 & 540 & $\mathrm{II}\left(2_{6}^{+2} 3^{-3} 5^{-1}\right)$ & 2 & 540 & $\mathrm{I}\left(2_{\mathrm{II}}^{+2} 3^{+3} 5^{-1}\right)$ \\
\hline 540 & $\mathrm{I}\left(2_{\mathrm{II}}^{+2} 3^{-1} 9^{+1} 5^{-1}\right)$ & 4 & 540 & $\mathrm{I}\left(2_{\mathrm{II}}^{+2} 3^{+3} 5^{+1}\right)$ & 2 & 540 & $\mathrm{I}\left(2_{\mathrm{II}}^{+2} 3^{-3} 5^{-1}\right)$ \\
\hline 540 & $\mathrm{I}\left(4_{7}^{+1} 3^{-3} 5^{+1}\right)$ & 2 & 540 & $\mathrm{I}\left(4_{1}^{+1} 3^{-3} 5^{-1}\right)$ & 3 & 567 & $\mathrm{I}\left(3^{+2} 9^{-1} 7^{-1}\right)$ \\
\hline 576 & $\mathrm{I}\left(2_{2}^{+2} 16_{5}^{-1} 3^{+2}\right)$ & 3 & 576 & $\mathrm{I}\left(2_{\mathrm{II}}^{+2} 16_{5}^{-1} 3^{+2}\right)$ & 2 & 576 & $\mathrm{I}\left(2_{2}^{+2} 16_{3}^{-1} 3^{+2}\right)$ \\
\hline 576 & $\mathrm{I}\left(2_{\mathrm{II}}^{+2} 16_{3}^{-1} 3^{+2}\right)$ & 2 & 576 & $\mathrm{I}\left(2_{\mathrm{II}}^{+2} 16_{7}^{+1} 3^{+2}\right)$ & 2 & 576 & $\mathrm{I}\left(4_{3}^{+3} 3^{-2}\right)$ \\
\hline 576 & $\mathrm{I}\left(4_{7}^{+3} 3^{+2}\right)$ & 2 & 576 & $\mathrm{I}\left(2_{2}^{+2} 16_{1}^{+1} 3^{+2}\right)$ & 3 & 576 & $\mathrm{I}\left(2_{\mathrm{II}}^{+2} 16_{1}^{+1} 3^{+2}\right)$ \\
\hline 576 & $\mathrm{I}\left(2_{2}^{+2} 16_{7}^{+1} 3^{+2}\right)$ & 3 & 576 & $\mathrm{I}\left(4_{1}^{+3} 3^{+2}\right)$ & 2 & 576 & $\mathrm{I}\left(4_{5}^{+3} 3^{-2}\right)$ \\
\hline 625 & $\mathrm{I}\left(5^{+2} 25^{-1}\right)$ & 2 & 648 & $\mathrm{I}\left(2_{3}^{+3} 3^{-2} 9^{+1}\right)$ & 3 & 648 & $\mathrm{I}\left(2_{3}^{+3} 3^{+2} 9^{+1}\right)$ \\
\hline 648 & $\mathrm{I}\left(2_{3}^{+3} 3^{+2} 9^{-1}\right)$ & 2 & 648 & $\mathrm{II}\left(2_{3}^{+1} 4_{1}^{+1} 3^{-2} 9^{-1}\right)$ & 3 & 648 & $\mathrm{II}\left(2_{7}^{+1} 4_{1}^{+1} 3^{+2} 9^{+1}\right)$ \\
\hline 648 & $\operatorname{II}\left(2_{7}^{+1} 4_{1}^{+1} 3^{+2} 9^{-1}\right)$ & 2 & 648 & $\mathrm{I}\left(2_{1}^{+1} 4_{1}^{+1} 3^{+2} 9^{+1}\right)$ & 3 & 648 & $\mathrm{I}\left(2_{1}^{+1} 4_{1}^{+1} 3^{+2} 9^{-1}\right)$ \\
\hline 675 & $\mathrm{I}\left(3^{-3} 5^{+2}\right)$ & 3 & 675 & $\mathrm{I}\left(3^{+3} 5^{-2}\right)$ & 3 & 686 & $\mathrm{I}\left(2_{1}^{+1} 7^{+3}\right)$ \\
\hline 729 & $\operatorname{II}\left(3^{-1} 9^{-1} 27^{-1}\right)$ & 3 & 729 & $\mathrm{I}\left(3^{+1} 9^{+1} 27^{-1}\right)$ & 1 & 729 & $\mathrm{I}\left(9^{-3}\right)$ \\
\hline
\end{tabular}




\begin{tabular}{|c|c|c|c|c|c|c|c|}
\hline 750 & $\mathrm{I}\left(2_{1}^{+1} 3^{+1} 5^{-3}\right)$ & 4 & 756 & $\mathrm{II}\left(2_{\mathrm{II}}^{+2} 3^{-3} 7^{-1}\right)$ & 2 & 756 & $\mathrm{II}\left(2_{\mathrm{II}}^{-2} 3^{+3} 7^{-1}\right)$ \\
\hline 768 & $\mathrm{I}\left(4_{\mathrm{II}}^{-2} 16_{3}^{-1} 3^{+1}\right)$ & 2 & 768 & $\mathrm{I}\left(4_{\mathrm{II}}^{+2} 16_{7}^{+1} 3^{-1}\right)$ & 1 & 768 & $\mathrm{I}\left(4_{2}^{+2} 16_{1}^{+1} 3^{+1}\right)$ \\
\hline 768 & $\mathrm{I}\left(4_{6}^{+2} 16_{1}^{+1} 3^{-1}\right)$ & 2 & 768 & $\mathrm{I}\left(4_{\mathrm{II}}^{-2} 16_{5}^{-1} 3^{-1}\right)$ & 1 & 768 & $\mathrm{I}\left(4_{\mathrm{II}}^{+2} 16_{1}^{+1} 3^{+1}\right)$ \\
\hline 768 & $\mathrm{I}\left(4_{2}^{+2} 16_{7}^{+1} 3^{+1}\right)$ & 3 & 768 & $\mathrm{I}\left(4_{6}^{+2} 16_{7}^{+1} 3^{-1}\right)$ & 2 & 768 & $\mathrm{I}\left(4_{\mathrm{II}}^{-2} 16_{1}^{+1} 3^{-1}\right)$ \\
\hline 768 & $\mathrm{I}\left(4_{\mathrm{II}}^{+2} 16_{5}^{-1} 3^{+1}\right)$ & 2 & 768 & $\mathrm{I}\left(4_{\mathrm{II}}^{-2} 16_{7}^{+1} 3^{+1}\right)$ & 2 & 768 & $\mathrm{I}\left(4_{\mathrm{II}}^{+2} 16_{3}^{-1} 3^{-1}\right)$ \\
\hline 784 & $\mathrm{II}\left(4_{\mathrm{II}}^{-2} 7^{+2}\right)$ & 1 & 810 & $\mathrm{I}\left(2_{1}^{+1} 3^{+2} 9^{-1} 5^{+1}\right)$ & 7 & 864 & $\mathrm{I}\left(2_{1}^{+1} 4_{\mathrm{II}}^{+2} 3^{+3}\right)$ \\
\hline 864 & $\mathrm{I}\left(2_{1}^{+1} 4_{2}^{+2} 3^{-3}\right)$ & 2 & 864 & $\mathrm{I}\left(2_{1}^{+1} 4_{\mathrm{II}}^{+2} 3^{-3}\right)$ & 1 & 864 & $\mathrm{I}\left(2_{1}^{+1} 4_{\mathrm{II}}^{+2} 3^{+1} 9^{-1}\right)$ \\
\hline 864 & $\mathrm{I}\left(2_{1}^{+1} 4_{2}^{+2} 3^{+3}\right)$ & 2 & 864 & $\mathrm{I}\left(2_{1}^{+1} 16_{1}^{+1} 3^{-3}\right)$ & 4 & 864 & $\mathrm{I}\left(2_{1}^{+1} 16_{7}^{+1} 3^{-3}\right)$ \\
\hline 864 & $\mathrm{I}\left(2_{1}^{+1} 16_{5}^{-1} 3^{-3}\right)$ & 3 & 864 & $\mathrm{I}\left(2_{1}^{+1} 16_{3}^{-1} 3^{-3}\right)$ & 3 & 900 & $\mathrm{II}\left(2_{\mathrm{II}}^{-2} 3^{-2} 5^{+2}\right)$ \\
\hline 900 & $\mathrm{II}\left(2_{\mathrm{II}}^{-2} 3^{+2} 5^{-2}\right)$ & 2 & 900 & $\mathrm{I}\left(2_{2}^{+2} 3^{+2} 5^{-2}\right)$ & 4 & 900 & $\mathrm{I}\left(4_{1}^{+1} 3^{+2} 5^{-2}\right)$ \\
\hline 900 & $\mathrm{I}\left(4_{7}^{+1} 3^{+2} 5^{-2}\right)$ & 4 & 960 & $\mathrm{I}\left(4_{5}^{+3} 3^{+1} 5^{-1}\right)$ & 2 & 960 & $\mathrm{I}\left(4_{3}^{+3} 3^{+1} 5^{+1}\right)$ \\
\hline 972 & $\mathrm{II}\left(2_{2}^{+2} 3^{-1} 9^{+2}\right)$ & 2 & 972 & $\operatorname{II}\left(2_{6}^{+2} 3^{+1} 9^{-2}\right)$ & 1 & 972 & $\mathrm{II}\left(2_{6}^{+2} 3^{+1} 9^{+2}\right)$ \\
\hline 972 & $\mathrm{I}\left(2_{2}^{+2} 3^{-1} 9^{+2}\right)$ & 2 & 972 & $\mathrm{I}\left(2_{\mathrm{II}}^{+2} 3^{-1} 9^{+2}\right)$ & 1 & 972 & $\mathrm{I}\left(2_{\mathrm{II}}^{+2} 3^{-1} 9^{-2}\right)$ \\
\hline 972 & $\mathrm{I}\left(2_{2}^{+2} 3^{+1} 9^{+2}\right)$ & 2 & 972 & $\mathrm{I}\left(2_{\mathrm{II}}^{+2} 3^{+1} 9^{+2}\right)$ & 2 & 972 & $\mathrm{I}\left(4_{1}^{+1} 3^{+1} 9^{+2}\right)$ \\
\hline 972 & $\mathrm{I}\left(4_{7}^{+1} 3^{+1} 9^{-2}\right)$ & 2 & 972 & $\mathrm{I}\left(4_{7}^{+1} 3^{+1} 9^{+2}\right)$ & 2 & 1000 & $\mathrm{I}\left(2_{3}^{+3} 5^{-3}\right)$ \\
\hline 1000 & $\mathrm{II}\left(2_{7}^{+1} 4_{1}^{+1} 5^{+3}\right)$ & 2 & 1008 & $\operatorname{II}\left(4_{2}^{-2} 3^{+2} 7^{-1}\right)$ & 3 & 1008 & $\mathrm{II}\left(4_{6}^{-2} 3^{+2} 7^{+1}\right)$ \\
\hline 1008 & $\mathrm{I}\left(4_{\mathrm{II}}^{-2} 3^{+2} 7^{-1}\right)$ & 3 & 1008 & $\mathrm{I}\left(4_{\mathrm{II}}^{-2} 3^{+2} 7^{+1}\right)$ & 3 & 1024 & $\mathrm{I}\left(4_{\mathrm{II}}^{-2} 64_{1}^{+1}\right)$ \\
\hline 1024 & $\mathrm{I}\left(4_{7}^{+1} 16_{\mathrm{II}}^{-2}\right)$ & 2 & 1024 & $\mathrm{I}\left(4_{1}^{+1} 16_{2}^{+2}\right)$ & 3 & 1029 & $\mathrm{II}\left(3^{-1} 7^{-3}\right)$ \\
\hline 1029 & $\mathrm{I}\left(3^{-1} 7^{-3}\right)$ & 2 & 1053 & $\mathrm{II}\left(3^{+2} 9^{-1} 13^{-1}\right)$ & 4 & 1080 & $\mathrm{I}\left(2_{3}^{+3} 3^{+3} 5^{-1}\right)$ \\
\hline 1080 & $\mathrm{II}\left(2_{1}^{+1} 4_{1}^{+1} 3^{-3} 5^{+1}\right)$ & 4 & 1125 & $\mathrm{II}\left(3^{+2} 5^{-3}\right)$ & 1 & 1125 & $\operatorname{II}\left(3^{-2} 5^{+3}\right)$ \\
\hline 1125 & $\mathrm{I}\left(3^{+2} 5^{-3}\right)$ & 2 & 1125 & $\mathrm{I}\left(3^{-2} 5^{+3}\right)$ & 2 & 1188 & $\mathrm{II}\left(2_{\mathrm{II}}^{-2} 3^{+3} 11^{+1}\right)$ \\
\hline 1188 & $\mathrm{II}\left(2_{\mathrm{II}}^{-2} 3^{-3} 11^{-1}\right)$ & 2 & 1200 & $\mathrm{II}\left(4_{2}^{+2} 3^{-1} 5^{+2}\right)$ & 3 & 1200 & $\mathrm{II}\left(4_{2}^{+2} 3^{+1} 5^{-2}\right)$ \\
\hline 1200 & $\mathrm{I}\left(4_{\mathrm{II}}^{-2} 3^{-1} 5^{+2}\right)$ & 3 & 1200 & $\mathrm{I}\left(4_{\mathrm{II}}^{-2} 3^{+1} 5^{-2}\right)$ & 3 & 1215 & $\mathrm{I}\left(3^{+1} 9^{+2} 5^{+1}\right)$ \\
\hline 1280 & $\mathrm{I}\left(4_{\mathrm{II}}^{-2} 16_{5}^{-1} 5^{+1}\right)$ & 2 & 1280 & $\mathrm{I}\left(4_{\mathrm{II}}^{-2} 16_{3}^{-1} 5^{+1}\right)$ & 2 & 1280 & $\mathrm{I}\left(4_{2}^{+2} 16_{1}^{+1} 5^{+1}\right)$ \\
\hline 1280 & $\mathrm{I}\left(4_{\mathrm{II}}^{-2} 16_{7}^{+1} 5^{+1}\right)$ & 2 & 1280 & $\mathrm{I}\left(4_{\mathrm{II}}^{-2} 16_{1}^{+1} 5^{+1}\right)$ & 2 & 1280 & $\mathrm{I}\left(16_{2}^{+2} 5^{+1}\right)$ \\
\hline 1280 & $\mathrm{I}\left(16_{6}^{-2} 5^{+1}\right)$ & 10 & 1296 & $\mathrm{I}\left(2_{\mathrm{II}}^{+2} 4_{7}^{+1} 3^{+2} 9^{+1}\right)$ & 2 & 1296 & $\mathrm{I}\left(2_{2}^{+2} 4_{1}^{+1} 3^{+2} 9^{+1}\right)$ \\
\hline 1296 & $\mathrm{I}\left(2_{\mathrm{II}}^{+2} 4_{1}^{+1} 3^{+2} 9^{+1}\right)$ & 2 & 1296 & $\mathrm{II}\left(4_{\mathrm{II}}^{-2} 3^{+2} 9^{-1}\right)$ & 2 & 1296 & $\mathrm{II}\left(4_{\mathrm{II}}^{-2} 3^{+2} 9^{+1}\right)$ \\
\hline 1296 & $\mathrm{II}\left(4_{4}^{-2} 3^{-2} 9^{+1}\right)$ & 2 & 1296 & $\operatorname{II}\left(4_{4}^{-2} 3^{-2} 9^{-1}\right)$ & 2 & 1296 & $\mathrm{I}\left(4_{\mathrm{II}}^{-2} 3^{-2} 9^{+1}\right)$ \\
\hline 1296 & $\mathrm{I}\left(4_{\mathrm{II}}^{-2} 3^{-2} 9^{-1}\right)$ & 2 & 1296 & $\operatorname{II}\left(4_{\mathrm{II}}^{+2} 3^{+2} 9^{-1}\right)$ & 2 & 1296 & $\mathrm{II}\left(4_{0}^{+2} 3^{+2} 9^{+1}\right)$ \\
\hline 1296 & $\mathrm{I}\left(4_{\mathrm{II}}^{+2} 3^{+2} 9^{+1}\right)$ & 3 & 1296 & $\mathrm{I}\left(4_{0}^{+2} 3^{+2} 9^{+1}\right)$ & 4 & 1296 & $\mathrm{I}\left(4_{2}^{+2} 3^{-2} 9^{+1}\right)$ \\
\hline 1296 & $\mathrm{I}\left(4_{2}^{+2} 3^{-2} 9^{-1}\right)$ & 2 & 1296 & $\mathrm{I}\left(4_{6}^{+2} 3^{+2} 9^{-1}\right)$ & 2 & 1296 & $\mathrm{I}\left(4_{6}^{+2} 3^{+2} 9^{+1}\right)$ \\
\hline 1296 & $\mathrm{I}\left(4_{2}^{+2} 3^{+2} 9^{-1}\right)$ & 2 & 1296 & $\mathrm{I}\left(4_{2}^{+2} 3^{+2} 9^{+1}\right)$ & 2 & 1296 & $\mathrm{I}\left(4_{6}^{+2} 3^{-2} 9^{+1}\right)$ \\
\hline 1296 & $\mathrm{I}\left(4_{6}^{+2} 3^{-2} 9^{-1}\right)$ & 2 & 1372 & $\mathrm{II}\left(2_{6}^{+2} 7^{+3}\right)$ & 2 & 1372 & $\mathrm{I}\left(2_{\mathrm{II}}^{+2} 7^{+3}\right)$ \\
\hline 1372 & $\mathrm{I}\left(4_{1}^{+1} 7^{+3}\right)$ & 3 & 1440 & $\mathrm{I}\left(2_{1}^{+1} 4_{\mathrm{II}}^{+2} 3^{+2} 5^{+1}\right)$ & 4 & 1440 & $\mathrm{I}\left(2_{1}^{+1} 4_{\mathrm{II}}^{+2} 3^{+2} 5^{-1}\right)$ \\
\hline 1500 & $\mathrm{II}\left(2_{2}^{+2} 3^{+1} 5^{+3}\right)$ & 3 & 1500 & $\mathrm{II}\left(2_{2}^{+2} 3^{-1} 5^{-3}\right)$ & 2 & 1500 & $\mathrm{II}\left(2_{6}^{+2} 3^{-1} 5^{+3}\right)$ \\
\hline 1500 & $\mathrm{I}\left(2_{\mathrm{II}}^{+2} 3^{+1} 5^{+3}\right)$ & 2 & 1500 & $\mathrm{I}\left(2_{\mathrm{II}}^{+2} 3^{-1} 5^{-3}\right)$ & 3 & 1500 & $\mathrm{I}\left(2_{\mathrm{II}}^{+2} 3^{+1} 5^{-3}\right)$ \\
\hline 1500 & $\mathrm{I}\left(4_{7}^{+1} 3^{-1} 5^{-3}\right)$ & 3 & 1500 & $\mathrm{I}\left(4_{1}^{+1} 3^{-1} 5^{+3}\right)$ & 2 & 1536 & $\mathrm{I}\left(2_{1}^{+1} 16_{2}^{+2} 3^{+1}\right)$ \\
\hline 1536 & $\mathrm{I}\left(2_{1}^{+1} 16_{0}^{+2} 3^{+1}\right)$ & 6 & 1536 & $\mathrm{I}\left(2_{1}^{+1} 16_{6}^{+2} 3^{+1}\right)$ & 3 & 1536 & $\mathrm{I}\left(2_{1}^{+1} 16_{4}^{-2} 3^{+1}\right)$ \\
\hline 1536 & $\mathrm{I}\left(2_{1}^{+1} 16_{2}^{-2} 3^{+1}\right)$ & 4 & 1536 & $\mathrm{I}\left(2_{1}^{+1} 16_{6}^{-2} 3^{+1}\right)$ & 3 & 1568 & $\mathrm{I}\left(2_{1}^{+1} 4_{\mathrm{II}}^{+2} 7^{+2}\right)$ \\
\hline
\end{tabular}




\begin{tabular}{|c|c|c|c|c|c|c|c|}
\hline 1620 & $\operatorname{II}\left(2_{\mathrm{II}}^{+2} 3^{-2} 9^{-1} 5^{+1}\right)$ & 3 & 1620 & $\mathrm{II}\left(2_{\mathrm{II}}^{+2} 3^{+2} 9^{+1} 5^{-1}\right)$ & 2 & 1620 & $\mathrm{II}\left(2_{\mathrm{II}}^{+2} 3^{+2} 9^{-1} 5^{-1}\right)$ \\
\hline 1620 & $\mathrm{II}\left(2_{\mathrm{II}}^{-2} 3^{+2} 9^{+1} 5^{+1}\right)$ & 2 & 1620 & $\mathrm{II}\left(2_{\mathrm{II}}^{-2} 3^{-2} 9^{+1} 5^{-1}\right)$ & 3 & 1620 & $\mathrm{II}\left(2_{\mathrm{II}}^{-2} 3^{+2} 9^{-1} 5^{+1}\right)$ \\
\hline 701 & $\mathrm{II}\left(3^{-1} 9^{+2} 7^{+1}\right)$ & 3 & 1728 & $\mathrm{I}\left(2_{2}^{+2} 16_{7}^{+1} 3^{+3}\right)$ & 3 & 1728 & $\mathrm{I}\left(2_{\mathrm{II}}^{+2} 16_{7}^{+1} 3^{+3}\right)$ \\
\hline 1728 & $\mathrm{I}\left(2_{2}^{+2} 16_{1}^{+1} 3^{-3}\right)$ & 2 & 1728 & $\mathrm{I}\left(4_{3}^{+3} 3^{-3}\right)$ & 1 & 1728 & $\mathrm{I}\left(4_{3}^{+3} 3^{+1} 9^{-1}\right)$ \\
\hline 1728 & $\mathrm{I}\left(4_{3}^{+3} 3^{+1} 9^{+1}\right)$ & 2 & 1728 & $\mathrm{I}\left(4_{7}^{+3} 3^{+3}\right)$ & 2 & 1728 & $\mathrm{I}\left(2_{\mathrm{II}}^{+2} 16_{1}^{+1} 3^{+1} 9^{+1}\right)$ \\
\hline 1728 & $\mathrm{I}\left(2_{2}^{+2} 16_{7}^{+1} 3^{-3}\right)$ & 2 & 1728 & $\mathrm{I}\left(4_{1}^{+3} 3^{-3}\right)$ & 1 & 1728 & $\mathrm{I}\left(4_{1}^{+3} 3^{+1} 9^{+1}\right)$ \\
\hline 1728 & $\mathrm{I}\left(4_{5}^{+3} 3^{+3}\right)$ & 2 & 1728 & $\mathrm{I}\left(2_{2}^{+2} 16_{1}^{+1} 3^{+3}\right)$ & 3 & 1728 & $\mathrm{I}\left(2_{\mathrm{II}}^{+2} 16_{5}^{-1} 3^{+1} 9^{+1}\right)$ \\
\hline 1728 & $\mathrm{I}\left(2_{2}^{+2} 16_{3}^{-1} 3^{-3}\right)$ & 2 & 1728 & $\mathrm{I}\left(2_{2}^{+2} 16_{5}^{-1} 3^{+3}\right)$ & 3 & 1728 & $\mathrm{I}\left(2_{2}^{+2} 16_{3}^{-1} 3^{+3}\right)$ \\
\hline 1728 & $\mathrm{I}\left(2_{\mathrm{II}}^{+2} 16_{3}^{-1} 3^{+3}\right)$ & 2 & 1728 & $\mathrm{I}\left(2_{2}^{+2} 16_{5}^{-1} 3^{-3}\right)$ & 2 & 1728 & $\mathrm{II}\left(4_{7}^{+1} 16_{7}^{+1} 3^{-3}\right)$ \\
\hline 1764 & $\mathrm{II}\left(2_{\mathrm{II}}^{-2} 3^{+2} 7^{+2}\right)$ & 2 & 1792 & $\mathrm{I}\left(4_{\mathrm{II}}^{-2} 16_{7}^{+1} 7^{+1}\right)$ & 3 & 1792 & $\mathrm{I}\left(4_{\mathrm{II}}^{-2} 16_{3}^{-1} 7^{+1}\right)$ \\
\hline 1875 & $\mathrm{I}\left(3^{+1} 5^{-2} 25^{-1}\right)$ & 5 & 1944 & $\mathrm{I}\left(2_{3}^{+3} 3^{-1} 9^{+2}\right)$ & 2 & 1944 & $\mathrm{II}\left(2_{5}^{+1} 4_{1}^{+1} 3^{+1} 9^{+2}\right)$ \\
\hline 2000 & $\mathrm{I}\left(2_{2}^{+2} 4_{1}^{+1} 5^{+3}\right)$ & 3 & 2000 & $\mathrm{II}\left(4_{0}^{+2} 5^{+3}\right)$ & 2 & 2000 & $\mathrm{I}\left(4_{2}^{+2} 5^{-3}\right)$ \\
\hline 2000 & $\mathrm{I}\left(4_{6}^{+2} 5^{+3}\right)$ & 2 & 2000 & $\mathrm{I}\left(4_{2}^{+}\right.$ & 2 & 2000 & $\mathrm{I}\left(4_{6}^{+}\right.$ \\
\hline 2000 & $\mathrm{I}\left(4_{\mathrm{II}}^{-2} 5^{+3}\right)$ & 2 & 2000 & $\mathrm{I}\left(16_{1}^{+1} 5^{+3}\right)$ & 5 & 2000 & $\mathrm{I}\left(16_{5}^{-1} 5^{+3}\right)$ \\
\hline 2160 & $\mathrm{II}\left(4_{2}^{-2} 3^{-3} 5^{+1}\right)$ & . & 2160 & $\mathrm{II}\left(4_{2}^{-2} 3^{+1} 9^{-1} 5^{+1}\right)$ & 4 & 2160 & $\mathrm{II}\left(4_{6}^{-2} 3^{+3} 5^{+1}\right)$ \\
\hline 2160 & $\mathrm{II}\left(4_{6}^{-2} 3^{-3} 5^{-1}\right)$ & 2 & 2160 & $\mathrm{I}\left(4_{\mathrm{II}}^{-2} 3^{-3} 5^{+1}\right)$ & 1 & 2160 & $\mathrm{I}\left(4_{\mathrm{II}}^{-2} 3^{+1} 9^{-1} 5^{+1}\right)$ \\
\hline 2160 & $\mathrm{I}\left(4_{\mathrm{II}}^{-2} 3^{+3} 5^{+1}\right)$ & 3 & 2160 & $\mathrm{I}\left(4_{\mathrm{II}}^{-2} 3^{-3} 5^{-1}\right)$ & 2 & 2250 & $\mathrm{I}\left(2_{1}^{+1} 3^{+2} 5^{+3}\right)$ \\
\hline 2250 & $\mathrm{I}\left(2_{1}^{+1} 3^{+2} 5^{-3}\right)$ & 4 & 2268 & $\mathrm{II}\left(2_{2}^{+2} 3^{+2} 9^{-1} 7^{-1}\right)$ & 4 & 2268 & $\mathrm{I}\left(2_{\mathrm{II}}^{+2} 3^{+2} 9^{+1} 7^{-1}\right)$ \\
\hline 2304 & $\mathrm{I}\left(4_{\mathrm{II}}^{-2} 16_{1}^{+1} 3^{-2}\right)$ & 2 & 2304 & $\mathrm{I}\left(4_{\mathrm{II}}^{+2} 16_{5}^{-1} 3^{+2}\right)$ & 2 & 2304 & $\mathrm{I}\left(4_{\mathrm{II}}^{-2} 16_{7}^{+1} 3^{-2}\right)$ \\
\hline 2304 & $\mathrm{I}\left(4_{\mathrm{II}}^{+2} 16_{3}^{-1} 3^{+2}\right)$ & 2 & 2304 & $\mathrm{I}\left(4_{\mathrm{II}}^{-2} 16_{3}^{-1} 3^{-2}\right)$ & 2 & 2304 & $\left.3_{7}^{+1} 3^{+2}\right)$ \\
\hline 2304 & $\mathrm{I}\left(4_{6}^{+2} 16_{1}^{+1} 3^{+2}\right)$ & 3 & 2304 & $\mathrm{I}\left(4_{\mathrm{II}}^{-2} 16_{5}^{-1} 3^{-2}\right)$ & 2 & 2304 & $\mathrm{I}\left(4_{\mathrm{II}}^{+2} 16_{1}^{+1} 3^{+2}\right)$ \\
\hline 2304 & $\mathrm{I}\left(4_{2}^{+2} 16_{7}^{+1} 3^{+2}\right)$ & 3 & 2500 & $\operatorname{II}\left(2_{\mathrm{II}}^{-2} 5^{+2} 25^{-1}\right)$ & 1 & 2500 & $\mathrm{II}\left(2_{\mathrm{II}}^{-2} 5^{+2} 25^{+1}\right)$ \\
\hline 2592 & $\mathrm{I}\left(2_{1}^{+1} 4_{\mathrm{II}}^{+2} 3^{-2} 9^{-1}\right)$ & 3 & 2592 & $\mathrm{I}\left(2_{1}^{+1} 4_{2}^{+2} 3^{+2} 9^{+1}\right)$ & 3 & 2592 & $\mathrm{I}\left(2_{1}^{+1} 4_{2}^{+2} 3^{+2} 9^{-1}\right)$ \\
\hline 2592 & $\mathrm{I}\left(2_{1}^{+1} 4_{\mathrm{II}}^{+2} 3^{+2} 9^{+1}\right)$ & 2 & 2592 & $\mathrm{I}\left(2_{1}^{+1} 4_{\mathrm{II}}^{+2} 3^{+2} 9^{-1}\right)$ & 2 & 2700 & $\mathrm{II}\left(2_{6}^{+2} 3^{-3} 5^{+2}\right)$ \\
\hline 2700 & $\mathrm{II}\left(2_{6}^{+2} 3^{+3} 5^{-2}\right)$ & 3 & 2700 & $\mathrm{I}\left(2_{\mathrm{II}}^{+2} 3^{-3} 5^{-2}\right)$ & 3 & 2700 & $\mathrm{I}\left(2_{\mathrm{II}}^{+2} 3^{+3} 5^{+2}\right)$ \\
\hline 2744 & $\mathrm{I}\left(2_{3}^{+3} 7^{+3}\right)$ & 3 & 2744 & $\mathrm{II}\left(2_{5}^{+1} 4_{1}^{+1} 7^{+3}\right)$ & 3 & 2916 & $\mathrm{II}\left(2_{\mathrm{II}}^{-2} 3^{-1} 9^{+1} 27^{+1}\right)$ \\
\hline 2916 & $\mathrm{II}\left(2_{\mathrm{II}}^{-2} 3^{+1} 9^{+1} 27^{-1}\right)$ & 1 & 2916 & $\mathrm{II}\left(2_{\mathrm{II}}^{-2} 9^{-3}\right)$ & 1 & 2916 & $\mathrm{II}\left(2_{\mathrm{II}}^{-2} 3^{-1} 9^{-1} 27^{+1}\right)$ \\
\hline 2916 & $\mathrm{II}\left(2_{\mathrm{II}}^{-2} 3^{+1} 9^{-1} 27^{-1}\right)$ & 2 & 2916 & $\mathrm{II}\left(2_{\mathrm{II}}^{-2} 9^{+3}\right)$ & 1 & 3000 & $\mathrm{I}\left(2_{3}^{+3} 3^{-1} 5^{+3}\right)$ \\
\hline 3000 & $\mathrm{II}\left(2_{5}^{+1} 4_{1}^{+1} 3^{+1} 5^{-3}\right)$ & 4 & 3072 & $\mathrm{I}\left(4_{1}^{+1} 16_{\mathrm{II}}^{-2} 3^{-1}\right)$ & 2 & 3072 & $\mathrm{I}\left(4_{\mathrm{II}}^{-2} 64_{1}^{+1} 3^{-1}\right)$ \\
\hline 3087 & $\mathrm{I}\left(3^{+2} 7^{+3}\right)$ & 3 & 3087 & $\mathrm{I}\left(3^{+2} 7^{-3}\right)$ & 3 & 3240 & $\mathrm{I}\left(2_{3}^{+3} 3^{+2} 9^{+1} 5^{-1}\right)$ \\
\hline 3240 & $\mathrm{II}\left(2_{3}^{+1} 4_{1}^{+1} 3^{+2} 9^{-1} 5^{+1}\right)$ & 7 & 3375 & $\mathrm{I}\left(3^{+3} 5^{-3}\right)$ & 1 & 3375 & $\mathrm{I}\left(3^{-1} 9^{+1} 5^{-3}\right)$ \\
\hline 3375 & $\mathrm{I}\left(3^{+3} 5^{+3}\right)$ & 2 & 3375 & $\mathrm{I}\left(3^{-3} 5^{-3}\right)$ & 3 & 3456 & $\mathrm{I}\left(2_{1}^{+1} 4_{1}^{+1} 16_{1}^{+1} 3^{-3}\right)$ \\
\hline 3456 & $\mathrm{I}\left(2_{1}^{+1} 4_{1}^{+1} 16_{7}^{+1} 3^{-3}\right)$ & 6 & 3600 & $\mathrm{II}\left(4_{\mathrm{II}}^{-2} 3^{+2} 5^{+2}\right)$ & 2 & 3600 & $\mathrm{II}\left(4_{4}^{-2} 3^{+2} 5^{-2}\right)$ \\
\hline 3600 & $\mathrm{I}\left(4_{\mathrm{II}}^{-2} 3^{+2} 5^{-2}\right)$ & 4 & 3600 & $\mathrm{I}\left(4_{2}^{+2} 3^{+2} 5^{-2}\right)$ & 4 & 3600 & $\mathrm{I}\left(4_{6}^{+2} 3^{+2} 5^{-2}\right)$ \\
\hline 3780 & $\mathrm{II}\left(2_{\mathrm{II}}^{-2} 3^{+3} 5^{-1} 7^{-1}\right)$ & 3 & 3780 & $\mathrm{II}\left(2_{\mathrm{II}}^{-2} 3^{-3} 5^{+1} 7^{-1}\right)$ & 3 & 3840 & $\mathrm{I}\left(4_{\mathrm{II}}^{-2} 16_{7}^{+1} 3^{+1} 5^{+1}\right)$ \\
\hline 3840 & $\mathrm{I}\left(4_{\mathrm{II}}^{-2} 16_{1}^{+1} 3^{+1} 5^{-1}\right)$ & 2 & 3840 & $\mathrm{I}\left(4_{\mathrm{II}}^{-2} 16_{5}^{-1} 3^{+1} 5^{-1}\right)$ & 2 & 3840 & $\mathrm{I}\left(4_{\mathrm{II}}^{-2} 16_{3}^{-1} 3^{+1} 5^{+1}\right)$ \\
\hline 3888 & $\mathrm{I}\left(2_{2}^{+2} 4_{1}^{+1} 3^{+1} 9^{+2}\right)$ & 4 & 3888 & $\mathrm{I}\left(2_{\mathrm{II}}^{+2} 4_{1}^{+1} 3^{+1} 9^{+2}\right)$ & 3 & 3888 & $\mathrm{II}\left(4_{2}^{+2} 3^{-1} 9^{+2}\right)$ \\
\hline 3888 & $\mathrm{II}\left(4_{6}^{+2} 3^{+1} 9^{-2}\right)$ & 1 & 3888 & $\mathrm{II}\left(4_{6}^{+2} 3^{+1} 9^{+2}\right)$ & 1 & 3888 & $\mathrm{I}\left(4_{\mathrm{II}}^{+2} 3^{-1} 9^{+2}\right)$ \\
\hline 3888 & $\mathrm{I}\left(4_{2}^{+2} 3^{+1} 9^{+2}\right)$ & 4 & 3888 & $\mathrm{I}\left(4_{\mathrm{II}}^{+2} 3^{+1} 9^{+2}\right)$ & 2 & 3888 & $\mathrm{I}\left(4_{0}^{+2} 3^{+1} 9^{+2}\right)$ \\
\hline
\end{tabular}




\begin{tabular}{|c|c|c|c|c|c|c|c|}
\hline 888 & $\operatorname{II}\left(4_{2}^{-2} 3^{-1} 9^{+2}\right)$ & 2 & 3888 & $\operatorname{II}\left(4_{6}^{-2} 3^{+1} 9^{+2}\right)$ & 2 & 3888 & $\mathrm{I}\left(4_{\mathrm{II}}^{-2} 3^{-1} 9^{+2}\right)$ \\
\hline 3888 & $\mathrm{I}\left(4_{\mathrm{II}}^{-2} 3^{+1} 9^{-2}\right)$ & 1 & 3888 & $\mathrm{I}\left(4_{\mathrm{II}}^{-2} 3^{+1} 9^{+2}\right)$ & 1 & 3969 & $\mathrm{I}\left(3^{+2} 9^{-1} 7^{+2}\right)$ \\
\hline 3993 & $\mathrm{I}\left(3^{-1} 11^{-3}\right)$ & 2 & 4000 & $\mathrm{I}\left(2_{1}^{+1} 4_{\mathrm{II}}^{+2} 5^{+3}\right)$ & 2 & 4096 & $\mathrm{I}\left(16_{7}^{-3}\right)$ \\
\hline 4096 & $\mathrm{I}\left(16_{3}^{+3}\right)$ & 2 & 4116 & $\mathrm{II}\left(2_{\mathrm{II}}^{+2} 3^{-1} 7^{-3}\right)$ & 2 & 4116 & $\mathrm{II}\left(2_{\mathrm{II}}^{-2} 3^{+1} 7^{-3}\right)$ \\
\hline 4320 & $\mathrm{I}\left(2_{1}^{+1} 4_{\mathrm{II}}^{+2} 3^{-3} 5^{+1}\right)$ & 4 & 4500 & $\mathrm{II}\left(2_{\mathrm{II}}^{+2} 3^{+2} 5^{-3}\right)$ & 2 & 4500 & $\mathrm{II}\left(2_{\mathrm{II}}^{+2} 3^{-2} 5^{+3}\right)$ \\
\hline 4500 & $\mathrm{II}\left(2_{\mathrm{II}}^{-2} 3^{+2} 5^{+3}\right)$ & 2 & 4500 & $\mathrm{II}\left(2_{\mathrm{II}}^{-2} 3^{-2} 5^{-3}\right)$ & 2 & 4860 & $\mathrm{II}\left(2_{2}^{+2} 3^{+1} 9^{+2} 5^{+1}\right)$ \\
\hline 4860 & $\mathrm{I}\left(2_{\mathrm{II}}^{+2} 3^{-1} 9^{+2} 5^{-1}\right)$ & 4 & 4900 & $\mathrm{II}\left(2_{\mathrm{II}}^{-2} 5^{-2} 7^{+2}\right)$ & 2 & 5120 & $\mathrm{I}\left(4_{1}^{+1} 16_{2}^{+2} 5^{+1}\right)$ \\
\hline 5120 & $\mathrm{I}\left(4_{7}^{+1} 16_{\mathrm{II}}^{-2} 5^{+1}\right)$ & 5 & 5184 & $\mathrm{I}\left(2_{\mathrm{II}}^{+2} 16_{5}^{-1} 3^{+2} 9^{+1}\right)$ & 4 & 5184 & $\mathrm{I}\left(2_{2}^{+2} 16_{3}^{-1} 3^{+2} 9^{+1}\right)$ \\
\hline 5184 & $\mathrm{I}\left(2_{\mathrm{II}}^{+2} 16_{3}^{-1} 3^{+2} 9^{+1}\right)$ & 4 & 5184 & $\mathrm{I}\left(2_{\mathrm{II}}^{+2} 16_{7}^{+1} 3^{+2} 9^{+1}\right)$ & 4 & 5184 & $\mathrm{I}\left(4_{3}^{+3} 3^{-2} 9^{+1}\right)$ \\
\hline 5184 & $\mathrm{I}\left(4_{3}^{+3} 3^{-2} 9^{-1}\right)$ & 2 & 5184 & $\mathrm{I}\left(4_{7}^{+3} 3^{+2} 9^{+1}\right)$ & 3 & 5184 & $\mathrm{I}\left(2_{\mathrm{II}}^{+2} 16_{1}^{+1} 3^{+2} 9^{+1}\right)$ \\
\hline 5184 & $\mathrm{I}\left(2_{2}^{+2} 16_{7}^{+1} 3^{+2} 9^{+1}\right)$ & 4 & 5184 & $\mathrm{I}\left(4_{1}^{+3} 3^{+2} 9^{+1}\right)$ & 3 & 5184 & $\mathrm{I}\left(4_{5}^{+3} 3^{-2} 9^{+1}\right)$ \\
\hline 5184 & $\mathrm{I}\left(4_{5}^{+3} 3^{-2} 9^{-1}\right)$ & 2 & 5488 & $\operatorname{II}\left(4_{6}^{-2} 7^{+3}\right)$ & 2 & 5488 & $\mathrm{I}\left(4_{\mathrm{II}}^{-2} 7^{+3}\right)$ \\
\hline 6000 & $\mathrm{II}\left(4_{2}^{-2} 3^{+1} 5^{+3}\right)$ & 3 & 6000 & $\left.{ }^{-1} 5^{-3}\right)$ & 2 & 6000 & $\operatorname{II}\left(4_{6}^{-2} 3^{-1} 5^{+3}\right)$ \\
\hline 6000 & $\mathrm{I}\left(4_{\mathrm{II}}^{-2} 3^{+1} 5^{+3}\right)$ & 3 & 6000 & $\mathrm{I}\left(4_{\mathrm{II}}^{-2} 3^{-1} 5^{-3}\right)$ & 2 & 6000 & $\mathrm{I}\left(4_{\mathrm{II}}^{-2} 3^{-1} 5^{+3}\right)$ \\
\hline 6084 & $\mathrm{II}\left(2_{\mathrm{II}}^{-2} 3^{+2} 13^{-2}\right)$ & 2 & 6750 & $\mathrm{I}\left(2_{1}^{+1} 3^{+3} 5^{+3}\right)$ & 4 & 6912 & $\mathrm{I}\left(4_{\mathrm{II}}^{-2} 16_{3}^{-1} 3^{-3}\right)$ \\
\hline 6912 & $\mathrm{I}\left(4_{\mathrm{II}}^{-2} 16_{3}^{-1} 3^{+1} 9^{-1}\right)$ & 2 & 6912 & $\mathrm{I}\left(4_{\mathrm{II}}^{-2} 16_{3}^{-1} 3^{+1} 9^{+1}\right)$ & 2 & 6912 & $\mathrm{I}\left(4_{\mathrm{II}}^{+2}\right.$ \\
\hline 6912 & $\mathrm{I}\left(4_{2}^{+2} 16_{1}^{+1} 3^{-3}\right)$ & 2 & 6912 & $\mathrm{I}\left(4_{6}^{+2} 16_{1}^{+1} 3^{+3}\right)$ & 3 & 6912 & $\mathrm{I}\left(4_{\mathrm{II}}^{-}\right.$ \\
\hline 6912 & $\mathrm{I}\left(4_{\mathrm{II}}^{+2} 16_{1}^{+1} 3^{-3}\right)$ & 1 & 6912 & $\mathrm{I}\left(4_{\mathrm{II}}^{+2} 16_{1}^{+1} 3^{+1} 9^{+1}\right)$ & 4 & 6912 & $\mathrm{I}\left(4_{2}^{+2}\right.$ \\
\hline 6912 & $\mathrm{I}\left(4_{6}^{+2} 16_{7}^{+1} 3^{+3}\right)$ & 3 & 6912 & $\mathrm{I}\left(4_{\mathrm{II}}^{-2} 16_{1}^{+1} 3^{+3}\right)$ & 2 & 6912 & $\left.5^{-1} 3^{-3}\right)$ \\
\hline 6912 & $\mathrm{I}\left(4_{\mathrm{II}}^{+2} 16_{5}^{-1} 3^{+1} 9^{+1}\right)$ & 4 & 6912 & $\mathrm{I}\left(4_{\mathrm{II}}^{-2} 16\right.$ & 1 & 6912 & $\mathrm{I}\left(4_{\mathrm{II}}^{-2} 1\right.$ \\
\hline 6912 & $\mathrm{I}\left(4_{\mathrm{II}}^{-2} 16_{7}^{+1} 3^{+1} 9^{+1}\right)$ & 2 & 6912 & $\mathrm{I}\left(4_{\mathrm{II}}^{+2} 1\right.$ & 2 & 7500 & $\mathrm{II}\left(2_{2}^{+2} 3^{+1} 5^{-2} 25^{-1}\right)$ \\
\hline 7500 & $\mathrm{I}\left(2_{\mathrm{II}}^{+2} 3^{-1} 5^{-2} 25^{+1}\right)$ & 5 & 7776 & $\mathrm{I}\left(2_{1}^{+1} 4_{\mathrm{II}}^{+2} 3^{+1} 9^{+2}\right)$ & 2 & 8000 & $\mathrm{I}\left(4_{5}^{+3} 5^{+3}\right)$ \\
\hline 8000 & $\mathrm{I}\left(2_{2}^{+2} 16_{1}^{+1} 5^{+3}\right)$ & 6 & 8000 & $\mathrm{I}\left(4_{3}^{+3} 5^{+3}\right)$ & 2 & 8000 & $\mathrm{I}\left(2_{2}^{+2} 16_{5}^{-1} 5^{+3}\right)$ \\
\hline 8000 & $\mathrm{II}\left(4_{7}^{+1} 16_{1}^{+1} 5^{+3}\right)$ & 5 & 8000 & $\mathrm{I}\left(4_{1}^{+1} 16_{1}^{+1} 5^{+3}\right)$ & 10 & 8100 & $\mathrm{II}\left(2_{\mathrm{II}}^{-2} 3^{-2} 9^{+1} 5^{+2}\right)$ \\
\hline 8100 & $\mathrm{II}\left(2_{\mathrm{II}}^{-2} 3^{+2} 9^{-1} 5^{-2}\right)$ & 3 & 8100 & $\mathrm{II}\left(2_{\mathrm{II}}^{-2} 3^{-2} 9^{-1} 5^{+2}\right)$ & 2 & 8100 & $\mathrm{II}\left(2_{\mathrm{II}}^{-2} 3^{+2} 9^{+1} 5^{-2}\right)$ \\
\hline 3640 & $\mathrm{I}\left(4_{5}^{+3} 3^{-3} 5^{-1}\right)$ & 3 & 8640 & $\mathrm{I}\left(4_{3}^{+3} 3^{-3} 5^{+1}\right)$ & 2 & 9000 & $\mathrm{I}\left(2_{3}^{+3} 3^{+2} 5^{+3}\right)$ \\
\hline 9000 & $\mathrm{I}\left(2_{3}^{+3} 3^{+2} 5^{-3}\right)$ & 4 & 9000 & $\mathrm{II}\left(2_{3}^{+1} 4_{1}^{+1} 3^{+2} 5^{+3}\right)$ & 4 & 9000 & $\mathrm{II}\left(2_{7}^{+1} 4_{1}^{+1} 3^{+2} 5^{-3}\right)$ \\
\hline 9072 & $\mathrm{II}\left(4_{2}^{-2} 3^{+2} 9^{-1} 7^{-1}\right)$ & 4 & 9072 & $\mathrm{I}\left(4_{\mathrm{II}}^{-2} 3^{+2} 9^{-1} 7^{-1}\right)$ & 4 & 9261 & $\mathrm{II}\left(3^{-3} 7^{+3}\right)$ \\
\hline 9261 & $\mathrm{II}\left(3^{-1} 9^{-1} 7^{-3}\right)$ & 3 & 9261 & $\mathrm{I}\left(3^{-3} 7^{+3}\right)$ & 2 & 10125 & $\mathrm{II}\left(3^{+2} 9^{+1} 5^{-3}\right)$ \\
\hline 0125 & $\left.{ }^{1} 5^{+3}\right)$ & 2 & 10125 & $\left.{ }^{-1} 5^{-3}\right)$ & 1 & 10125 & $\mathrm{I}\left(3^{+2} 9^{+1} 5^{-3}\right)$ \\
\hline 10125 & $\mathrm{I}\left(3^{-2} 9^{+1} 5^{+3}\right)$ & 3 & 10125 & $\mathrm{I}\left(3^{+2} 9^{-1} 5^{-3}\right)$ & 2 & 10500 & $\mathrm{II}\left(2_{\mathrm{II}}^{-2} 3^{+1} 5^{+3} 7^{-1}\right)$ \\
\hline 10500 & $\mathrm{II}\left(2_{\mathrm{II}}^{-2} 3^{-1} 5^{-3} 7^{-1}\right)$ & 3 & 10800 & $\mathrm{II}\left(4_{6}^{+2} 3^{-3} 5^{+2}\right)$ & 3 & 10800 & $\mathrm{II}\left(4_{6}^{+2} 3^{+3} 5^{-2}\right)$ \\
\hline 10800 & $\mathrm{I}\left(4_{\mathrm{II}}^{-2} 3^{-3} 5^{+2}\right)$ & 3 & 10800 & $\mathrm{I}\left(4_{\mathrm{II}}^{-2} 3^{+3} 5^{-2}\right)$ & 3 & 10976 & $\mathrm{I}\left(2_{1}^{+1} 4_{\mathrm{II}}^{+2} 7^{+3}\right)$ \\
\hline 11664 & $\mathrm{II}\left(4_{\mathrm{II}}^{-2} 3^{-1} 9^{-1} 27^{-1}\right)$ & 6 & 12000 & $\mathrm{I}\left(2_{1}^{+1} 4_{\mathrm{II}}^{+2} 3^{+1} 5^{-3}\right)$ & 4 & 12288 & $\mathrm{I}\left(16_{5}^{+3} 3^{-1}\right)$ \\
\hline 12288 & $\mathrm{I}\left(16_{1}^{-3} 3^{-1}\right)$ & 2 & 12348 & $\mathrm{II}\left(2_{2}^{+2} 3^{+2} 7^{+3}\right)$ & 3 & 12348 & $\mathrm{II}\left(2_{6}^{+2} 3^{+2} 7^{-3}\right)$ \\
\hline 12348 & $\mathrm{I}\left(2_{\mathrm{II}}^{+2} 3^{+2} 7^{+3}\right)$ & 3 & 12348 & $\mathrm{I}\left(2_{\mathrm{II}}^{+2} 3^{+2} 7^{-3}\right)$ & 3 & 12960 & $\mathrm{I}\left(2_{1}^{+1} 4_{\mathrm{II}}^{+2} 3^{+2} 9^{-1} 5^{+1}\right)$ \\
\hline 3500 & $\mathrm{II}\left(2_{2}^{+2} 3^{+3} 5^{-3}\right)$ & 1 & 13500 & $\mathrm{II}\left(2_{2}^{+2} 3^{-1} 9^{+1} 5^{-3}\right)$ & 4 & 13500 & $\mathrm{II}\left(2_{6}^{+2} 3^{+3} 5^{+3}\right)$ \\
\hline 3500 & $\mathrm{II}\left(2_{6}^{+2} 3^{-3} 5^{-3}\right)$ & 3 & 13500 & $\mathrm{I}\left(2_{\mathrm{II}}^{+2} 3^{-3} 5^{+3}\right)$ & 1 & 13500 & $\mathrm{I}\left(2_{\mathrm{II}}^{+2} 3^{+1} 9^{-1} 5^{+3}\right)$ \\
\hline 3500 & $\mathrm{I}\left(2_{\mathrm{II}}^{+2} 3^{+3} 5^{+3}\right)$ & 3 & 13500 & $\mathrm{I}\left(2_{\mathrm{II}}^{+2} 3^{-3} 5^{-3}\right)$ & 2 & 13500 & $\mathrm{I}\left(4_{7}^{+1} 3^{+3} 5^{-3}\right)$ \\
\hline
\end{tabular}




\begin{tabular}{|c|c|c|c|c|c|c|c|}
\hline 1500 & $\mathrm{I}\left(4_{1}^{+1} 3^{+3} 5^{+3}\right)$ & 3 & 3824 & $\mathrm{I}\left(2_{1}^{+1} 16_{2}^{+2} 3^{-3}\right)$ & 3 & 13824 & $\mathrm{I}\left(2_{1}^{+1} 16_{0}^{+2}\right.$ \\
\hline 13824 & $\mathrm{I}\left(2_{1}^{+1} 16_{6}^{+2} 3^{-3}\right)$ & 4 & 13824 & $\mathrm{I}\left(2_{1}^{+1} 16_{4}^{-2} 3^{-3}\right)$ & 6 & 13824 & $\mathrm{I}\left(2_{1}^{+1} 16_{2}^{-2} 3^{-3}\right)$ \\
\hline 824 & $\left(2_{1}^{+1} 16_{6}^{-2} 3^{-3}\right)$ & 4 & 4400 & $\mathrm{I}\left(4_{3}^{+3} 3^{+2} 5^{-2}\right)$ & 4 & 14400 & $\mathrm{I}\left(4_{5}^{+3} 3^{+}\right.$ \\
\hline 552 & $\mathrm{I}\left(4_{3}^{+3} 3^{+1} 9^{-2}\right)$ & 2 & 15552 & $\mathrm{I}\left(4_{3}^{+3} 3^{+1} 9^{+2}\right)$ & 2 & 15552 & $\mathrm{I}\left(2_{\mathrm{II}}^{+2} 16_{1}^{+1} 3^{+1} 9^{+2}\right)$ \\
\hline 15552 & $\mathrm{I}\left(4_{1}^{+3} 3^{+1} 9^{+2}\right)$ & $I$ & 15552 & $\mathrm{I}\left(2_{\mathrm{II}}^{+2} 16_{5}^{-1} 3^{+1} 9^{+2}\right)$ & 6 & 15876 & $\left.+29^{-1} 7^{+2}\right)$ \\
\hline 15876 & $\mathrm{I}\left(2_{\mathrm{II}}^{-2} 3^{+2} 9^{+1} 7^{+2}\right)$ & 2 & 15972 & $\mathrm{II}\left(2_{\mathrm{II}}^{-2} 3^{+1} 11^{+3}\right)$ & 2 & 15972 & $\mathrm{II}\left(2_{\mathrm{II}}^{-2} 3^{-1} 11^{-3}\right)$ \\
\hline 16384 & $\mathrm{I}\left(16_{\mathrm{II}}^{-2} 64_{3}^{-1}\right)$ & & 16875 & $\mathrm{I}\left(3^{+3} 5^{-2} 25^{+1}\right)$ & 0 & 19440 & $\mathrm{II}\left(4_{2}^{-2} 3^{+1}\right.$ \\
\hline 19440 & $\left(4_{\mathrm{II}}^{-2} 3\right.$ & 4 & 19652 & $\mathrm{II}\left(2_{\mathrm{II}}^{-2} 17^{+3}\right)$ & 2 & 19773 & \\
\hline 20250 & $\left.9^{+1} 5^{-3}\right)$ & 7 & 20480 & & 5 & 20480 & \\
\hline 20580 & $\mathrm{II}\left(2_{\mathrm{II}}^{-2} 3^{+1} 5^{+1} 7^{+3}\right)$ & & 20580 & $\mathrm{II}\left(2_{\mathrm{II}}^{-2} 3^{-1} 5^{-1} 7^{+}\right.$ & 3 & 20736 & $\mathrm{I}\left(4_{\mathrm{II}}^{-2}\right.$ \\
\hline 20736 & $\mathrm{I}\left(4_{\mathrm{II}}^{-2} 16_{1}^{+1} 3\right.$ & 2 & 20736 & $\mathrm{I}\left(4_{\mathrm{II}}^{+2} 16_{5}^{-1}\right.$ & 3 & 20736 & $\mathrm{I}\left(4_{\mathrm{II}}^{-2}\right.$ \\
\hline 20736 & $\mathrm{I}\left(4_{\mathrm{II}}^{-2} 1\right.$ & 2 & 20736 & $\mathrm{I}\left(4_{\mathrm{II}}^{+2} 16\right.$ & 3 & 20736 & $\mathrm{I}\left(4_{\mathrm{II}}^{-2}\right.$ \\
\hline 20736 & $\mathrm{I}\left(4_{\mathrm{II}}^{-2}\right.$ & 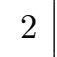 & 20736 & $\mathrm{I}\left(4_{\mathrm{II}}^{+2}\right.$ & 3 & 20736 & $\mathrm{I}\left(4_{\mathrm{II}}^{-}\right.$ \\
\hline 20736 & $\left.3^{-2} 9^{-1}\right)$ & 2 & 20736 & $\mathrm{I}\left(4_{\mathrm{II}}^{+2} 1\right.$ & 3 & 20736 & $\mathrm{I}\left(4_{2}^{+2}\right.$ \\
\hline 1952 & & 3 & 22500 & $\mathrm{II}\left(2_{\mathrm{II}}^{-2} 3^{+}\right.$ & 2 & 22500 & $\mathrm{II}\left(2_{\mathrm{II}}^{-2} 3\right.$ \\
\hline 24000 & $\mathrm{I}\left(4_{5}^{+}\right.$ & 2 & 24000 & & 3 & 27000 & \\
\hline 27000 & $\mathrm{I}\left(2_{5}^{+1} 4_{1}^{+1} 3^{+3} 5^{+3}\right.$ & 4 & 27648 & $\mathrm{I}\left(4_{\mathrm{I}}^{-}\right.$ & 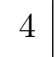 & 27648 & \\
\hline 27783 & $\left.{ }^{1} 7^{-3}\right)$ & 4 & 30000 & $\left.-225^{-1}\right)$ & 5 & 30000 & $\mathrm{I}\left(4_{\mathrm{II}}^{-2}\right.$ \\
\hline 30375 & & 4 & 32000 & $\mathrm{I}\left(4_{\mathrm{II}}^{-2} 1\right.$ & 2 & 32000 & \\
\hline 32000 & +3 ) & 0 & 32000 & & 2 & 32000 & \\
\hline 32000 & & 10 & 000 & & 10 & 0 & \\
\hline 34560 & $4_{\mathrm{II}}^{-2} 1$ & 3 & 34560 & $\mathrm{I}\left(4_{\mathrm{II}}^{-2}\right.$ & 3 & 34560 & $\mathrm{I}\left(4_{\mathrm{II}}^{-2}\right.$ \\
\hline 35937 & & 2 & 36000 & $\mathrm{I}\left(2_{1}^{+}\right.$ & 4 & 36000 & $\mathrm{I}\left(2_{1}^{+}\right.$ \\
\hline 37044 & $\mathrm{I}\left(2^{-}\right.$ & 2 & 37044 & & 2 & 40500 & $\mathrm{II}\left(2_{\mathrm{I}}^{+}\right.$ \\
\hline 40500 & $(2+2$ & 3 & & & 2 & & \\
\hline 40500 & $\left.5^{+3}\right)$ & 2 & 40500 & $\mathrm{II}\left(2_{\mathrm{I}}^{-}\right.$ & 2 & 49152 & $\mathrm{I}(16$ \\
\hline 49392 & $\left.7^{+3}\right)$ & 3 & 49392 & $\mathrm{II}($ & 3 & 49392 & \\
\hline 49392 & & 3 & 54000 & & 1 & 54000 & $\mathrm{II}(4$ \\
\hline 000 & $3-+3$ & 2 & 00 & $\mathrm{II}(4$ & 3 & 4000 & \\
\hline 54000 & $3)$ & 4 & 54000 & $\mathrm{I}(4$ & 2 & 4000 & \\
\hline 57600 & $\mathrm{I}\left(4_{\mathrm{II}}^{-2}\right.$ & 4 & 57600 & $\left.3^{+2} 5^{-2}\right)$ & 4 & 57600 & $\mathrm{I}\left(4_{\mathrm{II}}^{-2}\right.$ \\
\hline 57600 & $\mathrm{I}\left(4_{\mathrm{II}}^{-2}\right.$ & 4 & 62208 & $\mathrm{I}\left(4_{\mathrm{II}}^{-2}\right.$ & 2 & 62208 & $\mathrm{I}\left(4_{\mathrm{II}}^{-2}\right.$ \\
\hline 62208 & $\mathrm{I}\left(4_{\mathrm{II}}^{+2} 16\right.$ & 4 & 62208 & $\mathrm{I}\left(4_{\mathrm{II}}^{+2} 16_{5}^{-1} 3^{+1} 9^{+2}\right)$ & 4 & 62208 & $\mathrm{I}\left(4_{\mathrm{II}}^{-2}\right.$ \\
\hline 62208 & $\mathrm{I}\left(4_{\mathrm{II}}^{-2}\right.$ & 2 & 67500 & $\mathrm{II}\left(2_{6}^{+2}\right.$ & 5 & 67500 & $\mathrm{I}\left(2_{\mathrm{II}}^{+2}\right.$ \\
\hline 81000 & $\mathrm{I}\left(2_{3}^{+3}\right.$ & 7 & & $\left.3^{+2} 9^{+1} 5^{-3}\right)$ & 7 & 349 & $\left.7^{+2} 7^{-3}\right)$ \\
\hline 87808 & $\left.{ }^{1} 7^{+3}\right)$ & 3 & 87808 & & 3 & 94500 & $\left.3^{+3} 5^{+3} 7^{+1}\right)$ \\
\hline 94500 & $\mathrm{II}\left(2_{\mathrm{II}}^{-2} 3^{-3} 5^{-3} 7^{+1}\right)$ & 3 & 96000 & $\mathrm{I}\left(4_{\mathrm{II}}^{-2} 16_{7}^{+1} 3^{-1} 5^{-3}\right)$ & 3 & 96000 & $\mathrm{I}\left(4_{\mathrm{II}}^{-2} 16_{1}^{+1} 3^{-1} 5^{+3}\right)$ \\
\hline 96000 & $\left.3^{-1} 5^{+3}\right)$ & 2 & 96000 & & 3 & 108000 & \\
\hline 0592 & $\mathrm{I}\left(16_{3}^{+3} 3^{-3}\right)$ & 2 & 110592 & $\mathrm{I}\left(16_{7}^{-3} 3^{-3}\right)$ & 2 & 111132 & $\mathrm{II}\left(2_{6}^{+2} 3^{+}\right.$ \\
\hline
\end{tabular}


C. PRIMitive TOtALLY-REFLECTIVE GENERA OF DIMENSION 4

\begin{tabular}{lcr|ccc|ccc}
111132 & $\mathrm{I}\left(2_{\mathrm{II}}^{+2} 3^{+2} 9^{+1} 7^{-3}\right)$ & 4 & 121500 & $\mathrm{II}\left(2_{2}^{+2} 3^{-1} 9^{+2} 5^{-3}\right)$ & 4 & 121500 & $\mathrm{I}\left(2_{\mathrm{II}}^{+2} 3^{+1} 9^{+2} 5^{+3}\right)$ & 4 \\
128000 & $\mathrm{I}\left(4_{1}^{+1} 16_{2}^{+2} 5^{+3}\right)$ & 10 & 128000 & $\mathrm{I}\left(4_{7}^{+1} 16_{\mathrm{II}}^{-2} 5^{+3}\right)$ & 5 & 143748 & $\mathrm{II}\left(2_{\mathrm{II}}^{-2} 3^{+3} 11^{-3}\right)$ & 2 \\
143748 & $\mathrm{II}\left(2_{\mathrm{II}}^{-2} 3^{-3} 11^{+3}\right)$ & 2 & 177957 & $\mathrm{II}\left(3^{+2} 9^{-1} 13^{-3}\right)$ & 4 & 185220 & $\mathrm{II}\left(2_{\mathrm{II}}^{-2} 3^{+3} 5^{+1} 7^{-3}\right)$ & 3 \\
185220 & $\mathrm{II}\left(2_{\mathrm{II}}^{-2} 3^{-3} 5^{-1} 7^{-3}\right)$ & 3 & 216000 & $\mathrm{I}\left(4_{5}^{+3} 3^{+3} 5^{+3}\right)$ & 3 & 216000 & $\mathrm{I}\left(4_{3}^{+3} 3^{+3} 5^{-3}\right)$ & 2 \\
270000 & $\mathrm{II}\left(4_{6}^{+2} 3^{+3} 5^{-2} 25^{+1}\right)$ & 5 & 270000 & $\mathrm{I}\left(4_{\mathrm{II}}^{-2} 3^{+3} 5^{-2} 25^{+1}\right)$ & 5 & 324000 & $\mathrm{I}\left(2_{1}^{+1} 4_{\mathrm{II}}^{+2} 3^{+2} 9^{+1} 5^{-3}\right)$ & 7 \\
442368 & $\mathrm{I}\left(16_{\mathrm{II}}^{-2} 64_{3}^{-1} 3^{-3}\right)$ & 4 & 444528 & $\mathrm{II}\left(4_{6}^{-2} 3^{+2} 9^{-1} 7^{-3}\right)$ & 4 & 444528 & $\mathrm{I}\left(4_{\mathrm{II}}^{-2} 3^{+2} 9^{-1} 7^{-3}\right)$ & 4 \\
486000 & $\mathrm{II}\left(4_{2}^{-2} 3^{-1} 9^{+2} 5^{-3}\right)$ & 4 & 486000 & $\mathrm{I}\left(4_{\mathrm{II}}^{-2} 3^{-1} 9^{+2} 5^{-3}\right)$ & 4 & 512000 & $\mathrm{I}\left(16_{3}^{+3} 5^{+3}\right)$ & 5 \\
512000 & $\mathrm{I}\left(16_{7}^{-3} 5^{+3}\right)$ & 5 & 514500 & $\mathrm{II}\left(2_{\mathrm{II}}^{-2} 3^{+1} 5^{-3} 7^{-3}\right)$ & 3 & 514500 & $\mathrm{II}\left(2_{\mathrm{II}}^{-2} 3^{-1} 5^{+3} 7^{-3}\right)$ & 3 \\
864000 & $\mathrm{I}\left(4_{\mathrm{II}}^{-2} 16_{7}^{+1} 3^{+3} 5^{-3}\right)$ & 2 & 864000 & $\mathrm{I}\left(4_{\mathrm{II}}^{-2} 16_{1}^{+1} 3^{+3} 5^{+3}\right)$ & 3 & 864000 & $\mathrm{I}\left(4_{\mathrm{II}}^{-2} 16_{5}^{-1} 3^{+3} 5^{+3}\right)$ & 3 \\
864000 & $\mathrm{I}\left(4_{\mathrm{II}}^{-2} 16_{3}^{-1} 3^{+3} 5^{-3}\right)$ & 2 & 4630500 & $\mathrm{II}\left(2_{\mathrm{II}}^{-2} 3^{+3} 5^{-3} 7^{+3}\right)$ & 3 & 4630500 & $\mathrm{II}\left(2_{\mathrm{II}}^{-2} 3^{-3} 5^{+3} 7^{+3}\right)$ & 3
\end{tabular}

TABLE 1. Totally-reflective genera in dimension 4. 